\title{
PROBING THE INTERACTIONS BETWEEN DYE MOLECULES AND METALLIC NANOPARTICLES - IMPLICATIONS FOR SURFACE ENHANCED SPECTROSCOPIES
}

\section{BY}

\section{BRENDAN LIAM DARBY}

\author{
A thesis \\ submitted to the Victoria University of Wellington \\ in fulfilment of the requirements for the degree of \\ Doctor of Philosophy \\ in Physics
}

Victoria University of Wellington 2016 



\section{Abstract}

The work in this thesis focuses on improving the understanding of two key aspects of the interaction between dye molecules and metallic nanoparticles, with particular relevance to Surface Enhanced Raman Spectroscopy (SERS). This is manifested from two main branches of experimental work; the first is concerned with improving the reproducibility of SERS sample preparation using colloidal solutions while the second focuses on directly measuring the absorption spectra of commonly used dye molecules on the surface of colloidal silver nanoparticles.

In the first body of work of the thesis, a major step towards improving SERS in colloidal solutions is achieved by highlighting a crucial, but unnoticed possible source of error for such samples; by comparing average enhancement factor measurements on colloidal solutions prepared using different analyte (dye) dilution methods, it is shown that large dye dilution factors can cause extreme variations in nanoparticle coverage across the entire sample. This not only causes analyte-dependent enhancement factors (which is highly undesirable) but can also lead to false identification of single-molecule SERS experiments using the well established bi-analyte method.

The errors associated with large dilution factors are interpreted as a competition between dye diffusion and adsorption kinetics. Time dependent fluorescencequenching measurements and finite element modelling (FEM) in COMOSL show that in any system where adsorption competes with diffusion, large dilution factors should be avoided. A simple protocol of half-half dilutions of analytes is proposed as a standard method to be adopted when preparing colloidal solutions for SERS to ensure uniform distribution of analytes is achieved.

The second body of work is an experimental investigation of the modification of the energy levels of commonly used dye molecules adsorbed to spherical silver nanoparticles at sub-monolayer concentrations. Through the use of a novel integrating sphere setup, the absorption spectra of Rhodamine 6G, Nile Blue, Rhodamine 700 and Crystal Violet are successfully measured on the surface of silver colloids at ultra-low concentrations where dye-dye interactions are negligible. These results indicate that for most dyes, absorption spectra on 
the colloid surface are shifted and/or broadened with respect to the free dye in solution. In the most extreme case, a blue shift of almost $90 \mathrm{~nm}$ for Crystal Violet suggests a strong chemical interaction with the silver surface.

A Mie-theory shell model of dye-coated silver spheres is found to accurately reproduce the measured evolution of absorption spectra as the dye concentration on the colloid surface is increased but overestimates the enhancement in absorption, which is interpreted as a result of the adsoprtion geometry of dyes on the surface, not captured by the shell model.

Finally, through careful wavelength dependent SERS measurements, the SERS Raman excitation profile of Crystal Violet is measured and shown to be closely linked to the modified absorbance as obtained in the integrating sphere setup. A standard optical transform model for computing the Raman excitation profile from the modified absorbance is applied and gives good agreement with the measured SERS data. These results represent a direct indication of chemical modifications of resonant molecules used in SERS studies. 


\section{List of Publications}

[1] Darby, B. L., Auguié, B., Meyer, M., Pantoja, A. E., \& Le Ru, E. C. Modified optical absorption of molecules on metallic nanoparticles at submonolayer coverage. Nature Photonics 10(1), 40-45 (2016).

[2] Darby, B. L. \& Le Ru, E. C. Competition between molecular adsorption and diffusion: Dramatic consequences for SERS in colloidal solutions. J. Am. Chem. Soc. 136, 10965-10973 (2014).

[3] Darby, B. L., Etchegoin, P. G., \& Le Ru, E. C. Single-molecule surfaceenhanced Raman spectroscopy with nanowatt excitation. Physical Chemistry Chemical Physics 16(43), 23895-23899 (2014). 



\section{Acknowledgements}

$\mathrm{A} \mathrm{PhD}$ at the best of times seems a daunting endeavour, yet it is at the same time a deeply rewarding and memorable experience. I am truly grateful to those who have been on this journey with me for the past 3 years and here I would like to express my thanks to them. In the same way one could describe both New Zealand and the Raman Lab, such will be my memory of my PhD: unique.

First and foremost I would like to thank my supervisor, Eric Le Ru, without whom this thesis would not be possible. Eric is an inspiring individual, in both academic pursuits and normal life, and I am privileged to have had the opportunity to learn from, laugh with and work alongside him over the course of my PhD. A trove of never ending knowledge matched only by a dedication to his students and the atmosphere of the Raman Lab, my time during would not have been the same without such an excellent supervisor to guide me. I can only hope my future superiors are equally as keen to jump off harbours and organise dodgeball events.

To my secondary supervisor Justin Hodgkiss with whom I have enjoyed a great interaction with over the years, I am grateful to for the many healthy scientific discussions we have had.

To the Raman team themselves, I could not have asked to work with a more talented, completely crazy, creative group of individuals that won many (self organised) stupid sporting events while still producing world class research; to Camille Artur, Walter Somerville, Baptiste Auguié and Chris Galloway, whom it was always a pleasure to work with.

I also thank the cohort of students, postdocs and other vagabonds associated with the Raman Lab during my time, who became known more as friends than colleagues; James McNulty, Eva Anton, Harry Warring, Conor Burke all of whom I have shared many great memories with inside and outside the lab.

I acknowledge all those who helped me scientifically from SCPS. It really is a testament to the collaborative nature of New Zealand when people are so willing to donate their time to helping a colleague; to Olly Pantoja for his 
immense help and advice in the lab. To Jim Johnstons group who were always happy to help a physicist lost in a chemistry lab and to Peter Northcote for lively discussions about our results. To the administrative staff of SCPS who always go above and beyond, and to Sarah Dadley and Kara Eaton who bailed me out of a few pickles during my MESA years.

A special thanks goes to Matthias Meyer, if only for morning coffee discussions about Frankie Boyle and tramping, but more so for your endless willingness to help in the lab and pass on knowledge. And my good friend Peter Hauer, who has been there from the humble beginnings to our not so humble ends, I always cherished our varied discussions and will always admire your ability to never fail in sending a new football email every week.

I must also thank the MacDiarmid Institute and Victoria University of Wellington for the opportunity to undertake my $\mathrm{PhD}$ in such a wonderful working environment, and in an equally wonderful country.

Finally, I would never be where I am today without the love and support of my family. I am so indebted to my brother Eoin, who took me under his wing when I first arrived. It can be a lonely place on the other side of the world and you made me feel at home from the day I arrived, something I will never forget. And finally to my parents, for your never ending support and encouragement. Every Skype brought with it a new boost of confidence, despite my desperate attempts at explaining what I do. Thank you for the love whose strength does not diminish with distance. 

"And the wise have pitied the fool

that hath striven to give a life

In the world of time and space

Among the bulks of actual things,

To a dream that was dreamed in the heart, And only the heart could hold.

$O$ wise men, riddle me this:

What if the dream come true?"

- Pádraig Pearse, from "The Fool" 


\section{Contents}

1 Introduction 1

1.1 A Brief History of SERS . . . . . . . . . . . . . . . . 2

1.1.1 Discovery of the SERS Effect . . . . . . . . . 2

1.1 .2 Single Molecule SERS . . . . . . . . . . . . . . . 3

1.1.3 SERS - Where are we now? . . . . . . . . . . 4

1.2 Colloidal Solutions for SERS . . . . . . . . . . . . . . 5

1.3 Thesis Motivation . . . . . . . . . . . . . . . . 8

1.4 Thesis Layout . . . . . . . . . . . . . . . . . . . . . 99 9

1.5 Scientific Outputs . . . . . . . . . . . . . . 10

2 Theoretical Background 11

2.1 Molecular Spectroscopy . . . . . . . . . . . . . . . . 11

2.1.1 Energy Levels of Molecules . . . . . . . . . . . . . . . 12

2.1.2 Optical Transitions in Molecules . . . . . . . . . . . . 13

2.1.3 Optical Transitions in Dye Molecules . . . . . . . . . 15

2.2 Raman Scattering . . . . . . . . . . . . . . . . . . . . 18 
2.2.1 The Classical Description of Raman Scattering . . . . . . 19

2.2.2 The Raman Spectrum . . . . . . . . . . . . . . . 20

2.2.3 The Raman Cross Section . . . . . . . . . . . . . . . 21

2.2.4 Resonance Raman Scattering . . . . . . . . . . . . 23

2.3 Surface Enhanced Spectroscopies _ . . . . . . . . . . . . 25

2.3.1 Optical Properties of Noble Metals . . . . . . . . . 25

2.3.2 Surface Plasmon Resonances - The Electrostatic approximation . . . . . . . . . . . . . . . . 28

2.3.3 The $E^{4}$ approximation in SERS . . . . . . . . . . . 32

2.3.4 Mie Theory .................. 33

2.3.5 Hot Spots in SERS Substrates . . . . . . . . . 36

3 Experimental Details $\quad 39$

3.1 SERS Experimental Setup . . . . . . . . . . . . . . 39

3.2 Colloidal Solutions as SERS substrates . . . . . . . . . . . 41

3.2.1 Lee and Meisel Silver Colloids . . . . . . . . . . . . 41

3.2.2 Nanocomposix Colloids . . . . . . . . . . . . . 43

3.2.3 Nanoparticle Concentration Estimations . . . . . . . . 44

3.2.4 Achieving SERS with colloidal nanoparticles . . . . . . 45

3.3 Common SERS Analytes . . . . . . . . . . . . . . . . . . . . 49

3.4 Experimental Determination of SERS Enhancement Factors . . 55

3.4.1 Average SERS Enhancement Factors . . . . . . . . 55 
3.4.2 SERS Spectra in Colloidal Solutions . . . . . . . . . 59

3.5 Single Molecule SERS . . . . . . . . . . . . . . . . . . 62

3.5.1 The Bi-analyte Technique . . . . . . . . . . 62

3.5.2 BiASERS with Isotopologues . . . . . . . . . . . 64

3.5.3 Single-molecule Enhancement Calculations . . . . . . . 66

4 Dilution Effects in Colloidal Solutions $\quad 69$

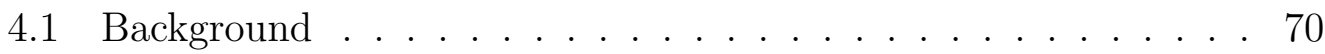

4.2 Experimental Conditions . . . . . . . . . . . . . . 71

4.3 Results . . . . . . . . . . . . . . . . . 72

4.3.1 The Effect of Dilution on Average Enhancement Factors 72

4.3.2 Diffusion vs Adsorption . . . . . . . . . . . . 76

4.3.3 Dynamics of Molecular Adsorption on Nanoparticles . . 78

4.3.4 Effects of Analyte Concentration on Aggregation of Nanoparticles . . . . . . . . . . . . . . . 80

4.3.5 Finite Element Modelling of Diffsusion . . . . . . . . . . 83

4.3.6 SEM Evidence for Dilution Effects . . . . . . . . . . 89

4.3.7 Effects on Single Molecule SERS . . . . . . . . . . . 92

4.4 Conclusion . . . . . . . . . . . . . . . . . . . . . . 99 
5 Integrating Spheres - Theory and Experimental Setup

5.1 The Theory of Integrating Spheres _ . . . . . . . . . . . 103

5.1.1 Reflectivity - Lambertian Surfaces . . . . . . . . . . . . . 104

5.1 .2 Sphere M-Factor . . . . . . . . . . . . 107

5.2 Absorption Spectroscopy in an Integrating Sphere . . . . . . . . 108

5.2 .1 Background . . . . . . . . . . . . . 108

5.2.2 Experimental Setup - Center Mounted Sample . . . . . . 109

5.2 .3 Sample Holder Design . . . . . . . . . . . . . . . 109

5.2.4 CMCIS Absorbance Corrections . . . . . . . . . . . . 111

5.2.5 Standard Absorbance Measurement Steps . . . . . . . 116

5.2.6 Differential Absorbance in the Integrating Sphere . . . . 117

5.2.7 Sphere Linearity Response . . . . . . . . . . . . 118

6 Modified Absorbance of Molecules Adsorbed to Metallic Nanoparticles

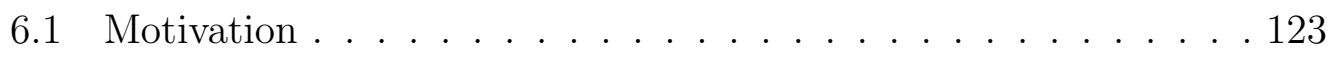

6.1.1 Chemical Enhancement in SERS . . . . . . . . 127

6.1.2 Wavelength Dependence of SERS at Resonance . . . . . 128

6.1.3 Strong Coupling between Plasmons and Molecules . . . . 133

6.2 The Challenge of Measuring Dye Surface Absorbance . . . . . . 137

6.2.1 Nanoparticle vs Dye Cross Sections . . . . . . . . . 139

6.2.2 Differential Extinction Artifacts . . . . . . . . . . . 140 
6.2.3 Concentration Dependence of Dye Layer Properties . . . 146

6.3 Experimental Results . . . . . . . . . . . . . . . . . . . . . . 149

6.3 .1 Sample Preparation . . . . . . . . . . . . . . . 149

6.3.2 Measuring Absorbance in a Turbid Medium . . . . . . . 151

6.3.3 Surface Absorbance Spectra . . . . . . . . . . . . 153

6.4 Agreement with Theory . . . . . . . . . . . . . . . 167

6.4.1 Enhanced Absorbance . . . . . . . . . . . . 167

6.4.2 Modified Absorbance in SERRS . . . . . . . . . . 171

6.5 Conclusion . . . . . . . . . . . . . . . . 176

$\begin{array}{llr}7 \text { Conclusion } & 179\end{array}$

A Polarizability of Dyes and Mie Theory Shell Model 185

A.1 Polarizability of dyes . . . . . . . . . . . . . 185

A.1.1 Link between polarizability and absorbance . . . . . . 185

A.1.2 Theoretical fits of bare polarizabilities . . . . . . . 186

A.1.3 DFT calculations of static polarizabilities . . . . . . . 189

A.1.4 Clausius-Mossotti Relationship for Dye Layers . . . . . . 190

A.1.5 Mie-Theory Model of Dye-Coated Spheres . . . . . . . . 191 



\section{Chapter 1}

\section{Introduction}

Surface enhanced Raman spectroscopy (SERS) has had a tumultuous history as a spectroscopic technique. Since its discovery in 1974 [1], its perception and popularity has varied significantly. From a peculiar phenomenon confined to the Surface Science community in the mid to late 1970s, to sparking interest across a large breadth of the field of electromagnetism in the 1980s, to dying off again due to its perceived niche applicability, to experiencing a complete renaissance of interest post 1997, after the first (yet controversial) report of single-molecule SERS (SM-SERS) detection, SERS has come and gone and come again. Given the early teething problems, since those seminal reports of SM-SERS by Kneipp [2] and Nie [3], interest in the technique has steadily increased throughout the 2000s. Coupled with the emergence of nanotechnology as a major pillar of global science in the past two decades, SERS as a go-to method for ultra-sensitive detection is steadily gaining traction to become a standard spectroscopic technique employed in a wide range of fields across research chemistry and fundamental spectroscopy. One can easily consult the literature and find the number of papers published related to SERS between 2014 and 2015 was almost $3000^{1}$. For thirty years now, SERS has been poised to revolutionize analytical chemistry, but it has yet to reach this status. The work in this thesis primarily builds on the vast amount of prior knowledge developed around SERS and clarifies a number of outstanding uncertainties surrounding, and hindering, the reliability and understanding of the technique.

\footnotetext{
${ }^{1}$ Web of Science, articles with either "Surface Enhanced Raman" or "SERS" in the title.
} 


\section{$1.1 \quad$ A Brief History of SERS}

\subsubsection{Discovery of the SERS Effect}

SERS was first discovered in 1974 when Fleischman et al. [1] were investigating the adsorption of a monolayer of species to a surface. The ability to detect Raman spectra of pyridine adsorbed on silver electrodes was originally assumed to be a direct result of an increase in surface area due to roughening of the electrodes and hence a larger number of molecules adsorbed at the surface. It was Van Duyne and Creighton [4,5] however, who independently attributed the abnormally high Raman signals to some property of the electrode surface not related to roughness acting to enhance the effective Raman scattering cross section of the adsorbed species. Van Duyne and Jeanmaire [4] calculated that the signal observed from the adsorbed pyridine was $10^{5}-10^{6}$ times larger than that of bulk pyridine. This clearly ruled out the idea that increased surface area was the cause of the enhancement.

The matter of the origin of the enhanced signal was far from resolved and over the course of the next few years a plethora of competing and contradictory theories emerged that attempted to develop an accurate model to describe the nature of this enhancement [6-8]. Moskovits [9] was the first to propose that the mechanism was purely an electromagnetic one. It was Moskovits' 1985 review article in which an extensive summary of the various theoretical models available at the time is provided, that sought to clear up the overwhelming contradictions present in the field. It was concluded, in what is now widely regarded as the main explanation for the SERS effect, that "SERS derives its enhancement almost entirely from molecular coupling to electromagnetic resonances of small, coupled metal particles" [10]. Thus the enhancement of the electric field arises from the interaction of the incident light with the localized surface plasmon resonance of the substrate. The excitation of these surface plasmons leads to an electromagnetic field at the at the surface of the metal that is much greater in intensity than the incident field. Molecules in the vicinity of this local field, can exhibit enhanced Raman scattering up to $10^{11}$ times that in the absence of the metal. The origin of the enhancement will be discussed in more detail in Chapter 3. 
The implications of SERS are immediately clear; it allows detection of the characteristic "fingerprint" Raman spectra from molecules at concentrations that would otherwise be practically impossible. Its applications in the fields of biochemistry, forensics, explosive detection and any other field that requires reliable identification of species at low concentrations are obvious. The massive increase in sensitivity provided by SERS opens the door for molecular specific analysis of systems previously out of reach of Raman spectroscopy. This was, in fact, one of the original attractions of SERS and since its discovery, the technique has been shown to be successful in a wide range of contexts. A number of these applications are discussed in detail in [11].

The discovery of SERS prompted not only an increased interest in the study of molecules adsorbed to surfaces; numerous fields such as classical electromagnetic theory, optical properties of small particles, surface plasmon generation and colloidal science all experienced a resurgence [10].

\subsubsection{Single Molecule SERS}

In 1997, Kneipp and Emory [2,3] both independently reported that detecting Raman signals from single Rhodamine 6G (RH6G) and Crystal Violet (CV) molecules using SERS was possible. These suggested (erroneously) [12] that enhancement factors as large as $10^{14}$ were achievable. The idea of Single Molecule SERS (SM SERS) has many attractions, the first of which is obvious; the well established technique of single molecule (SM) fluorescence detection $[13,14]$ is limited by its lack of molecular specificity. SM-SERS immediately brings a new level of sprectral information that could open up new applications beyond bio-physics and imaging [15] where SM-fluorescence is predominantly used. Secondly, SM SERS has the characteristics of the ultimate analytical technique; the unique detection of a single molecule pushes the boundaries of the analytical tools available today. As well as the potential practical applications of the technique, SM SERS is also of significant interest to fundamental research; it could possibly provide a better understanding of the SERS effect itself, where certain effects such as molecular orientation, are no longer "washed out" due to ensemble averaging that occurs at higher 
probe concentrations [16]. Recent studies have shown the promise of SMSERS for investigation of fundamental spectroscopic phenomena such as the homogenous broadening of spectral lineshapes [17] and the detection of natural isotopic substitution [18].

With these ideas in mind, it is clear to see why SM-SERS caused a second renaissance of SERS in general, sparking a renewed interest in a field that was in reality losing its original momentum since its discovery [19].

\subsubsection{SERS - Where are we now?}

Despite its widespread use in research and fundamental contexts, SERS is still often viewed outside these confines as a technique plagued with problems; contradictory claims (for example in terms of the SERS enhancement factors (EFs) [20], unresolved questions (illustrated for example by the debate between electromagnetic and chemical enhancements), uncontrolled large signal fluctuations, and irreproducibility [21]. The first two items in this list are perhaps intrinsic to any research endeavor, but the latter two are particular weaknesses for a technique that should be more than mature enough to be an essential part of the analytical chemists toolbox.

Procedures have been developed to avoid signal fluctuations and improve irreproducibility, at least in specific contexts. Efforts in this direction include rigorous definitions and quantification of the SERS EFs [12] and promising quantification/calibration studies [22-25], notably using internal standards [26]. The cause of the SERS fluctuations has also been better identified thanks to progress in single molecule SERS detection [27,28] and studies of the enhancement factor distribution $[29,30]$ and the closely related concept of hotspots [31], which are highly localized regions (a few $\mathrm{nm}$ ) where the SERS EF is extremely large [19]. The recognition of the presence of such hot-spots goes a long way in explaining the origin of the fluctuation/reproducibility problem [29,32], especially in view of the fact that hot-spot properties are extremely sensitive to minute variations in the underlying geometry (for example sub-nanometer changes in the gap between two metallic nanoparticles (NPs) [33]). 
Recent and ongoing studies will no doubt improve dramatically the ability to seriously address the SERS fluctuation/reproducibility issue, both for general analytical chemistry applications and for single-molecule or trace detection studies yet a large proportion of recent papers published in the field are still striving to produce SERS substrates that provide uniform and reproducible signals, highlighting that there still exist a number of outstanding issues associated with the technique.

The second, more subtle thorn in the side of SERS' emergence as a standard technique is the debate over the existence, and role, of chemical enhancements [34-36] in the SERS mechanism. Discussed in detail in Chapter 6, the original debate around the mechanism of the SERS enhancement has been a hotly contested topic that began in the early 1980s [37]. Today it is generally accepted that the majority of the enhancement arises from electromagnetic effects at the surface of the metallic substrate [38] and that chemical effects will contribute at most a factor of 100 to the overall enhancement [39]. This agreement among the field's researchers has gone a long way towards moving on from arguing over the subtle aspects of the technique, allowing much more robust applications of SERS to be developed across many fields. The "Chemical Enhancement" debate still is prevalent among the SERS literature, and the advances in both large scale theoretical modelling [40] (Time Dependent Density Functional Theory (TDDFT)) and control of nanostructure fabrication (colloidal synthesis [41], lithographically prepared substrates [42]) have been crucial in investigating this elusive phenomenon. It is natural to say that understanding this aspect of the SERS effect is the last piece of the puzzle in fully describing the fundamental mechanisms at play.

\subsection{Colloidal Solutions for SERS}

The work presented in this thesis solely uses colloidal solutions of metallic nanoparticles as the SERS substrate. As such an overview of colloidal solutions in the context of SERS is briefly discussed.

A quick glance at almost any recently published article related to SERS and one will find mention of the difficulty in obtaining quantitative, reliable 
and repeatable results from a SERS substrate. Although some aspects of these issues arise from the nature of the technique itself, being extremely sensitive to any change in the local environment of the analyte molecule (hot spot gap size, surface roughness, molecular orientation, thermal effects etc) the source, in general, can often be attributed to the use of colloidal solutions as SERS substrates. Despite their widespread use as the workhorse of the field since Lee and Meisel in the early 1980s [43], colloidal solutions have without doubt been a double edged sword for the field's development. On the one hand, the properties of colloids allow an impressive simplicity towards performing SERS experiments and probing the nature of molecule-nanoparticle interactions. Their ease of preparation, cheap cost of manufacture, relatively long term shelf life (on the order of six months to a year), and most importantly large SERS enhancements make them a hugely attractive option for many researchers looking to perform studies ranging from simple determination of signal enhancements for a given molecule all the way down to single molecule SERS experiments. Moreover, working in liquid brings significant advantages over dried nanostructures in terms of spectral statistics; the Brownian motion of the colloids allows one to probe the entire solution by simply acquiring large numbers of spectra, while the diffusive properties of solutions ensures that photobleaching of dye molecules is less of an issue than when working on dried substrates [44]. On the other hand, colloidal solutions, in particular those made from silver, are known to exhibit large signal fluctuations and results can often be very difficult to reproduce. These drawbacks arise from a number of factors, including batch to batch variation, polydispersity in particle size and shape along with sensitivity to oxidation (silver in particular) and the possible aggregation induced by analyte molecules themselves. Probably the most significant drawback of using colloidal solutions (especially with spherical nanoparticles) is the need to aggregate the particles to create hot-spots, regions of large electric field intensity described in Chapter 3, where the enormous signal enhancements originate from. Aggregation is normally induced by the addition of electrolyte [24], such as salts, to the colloids and is by definition an extremely difficult step to control.

To address the inherent problems associated with the traditional colloidal SERS approaches, many stategies have moved away from colloidal solutions and nano-lithographically prepared structures of gold and silver are becoming 
more common as SERS substrates [45,46]. Many still admire the colloidal approach for simplicity its applicability to a broad range of analytes/systems [47-49]. In this regard there has been a strong move towards removing the necessary aggregation step with spherical particles by synthesising non-spherical particles. In recent years, a plethora of silver and gold nanoparticle shapes have been successfully synthesised [50] and applied to SERS detection [51]. Examples range from nano-rods, stars, and triangles to bi-pyramids [32], all of which contain sharp features that act as electromagnetic hot-spots in place of the gap mode hot spots present in spherical nanoparticle solutions. More complicated architectures can be synthesised, such as core-shell particles with tunable plasmon resonances [52]. Another promising approach in the last 3-4 years has been to gain better control over the aggregation step required to create hot-spots by the use of DNA origami methods [53] and molecular linkers. This consists of developing a DNA architecture that essentially acts as a linking bridge between nanoparticles. Due to the well established methods in DNA linking, tailorable gap sizes between particles can be engineered to assemble hot spots of desired distances between nanoparticles [39]. The downsides to such approaches are the lower limit on gap sizes achievable (due to the minimum number of bases required for stable linking) and time consuming methods must be employed to obtain gaps small enough to produce large enhancements.

The past 5 years have seen the development of many promising alternative approaches to traditional colloidal SERS and the maturity of these techniques is impressive. There still however is significant interest in the traditional silver and gold, citrate reduced colloids whose advantages have been discussed. While the more advanced substrates using DNA origami are an excellent strategy for analytical applications of SERS, there is no doubt that traditional colloids still have their place among the SERS field. In particular, citrate reduced silver colloids still outperform other substrates for single molecule detection in solution and allow easy investigation of many fundamental aspects of SERS. From this point of view, much of the work in this thesis uses, and significantly improves the reliability of, these type of colloids. 


\subsection{Thesis Motivation}

With the considerations above in mind, the work in this thesis addresses two separate but closely linked aspects of SERS that have to date not been studied. The first body of work identifies a crucially important source of error and irreproducibility when performing SERS in colloidal solutions by demonstrating that different dilution methods of analytes into colloidal solutions can lead to drastically different outcomes for analyte coverage across the sample. This unexpected result has gone unnoticed up to now in the field and it is shown that unless accounted for, serious misinterpretations can be made for the determination of average enhancement factors (AEF), and more importantly for the analysis of SM-SERS detection.

Secondly, a novel experimental setup, consisting of an integrating sphere with a centrally mounted cuvette (CMCIS), was developed to allow, in conjunction with the new information provided by the dilution study, measurement of the enhanced and modified absorbance of commonly used dye molecules adsorbed to colloidal silver nanospheres. Direct measurement of this surface absorbance is a significant step towards understanding the chemical effect in SERS, particularly in the context of Surface Enhanced Resonant Raman Spectroscopy (SERRS). 


\subsection{Thesis Layout}

The layout of this thesis is as follows:

Chapter 2 presents a general theoretical background of all concepts relevant to the experimental results presented in later chapters. First is an overview of the relevant spectroscopic concepts including energy levels in molecules, Raman and Resonance Raman spectroscopy. The electromagnetic theory governing the SERS effect, including a brief description of Mie-theory methods for calculating the optical properties metallic particles is then discussed and definitions of SERS enhancement factors, both average and single molecule are introduced.

Chapter 3 presents a description of the general experimental details used in this thesis. Most of these details are relevant to Chapter 4, in particular the procedures for calculating average and single molecule SERS EFs, but some extend to the results of Chapter 6 .

Chapter 4 discusses the first part of the experimental section of the thesis, namely dilution effects in SERS experiments. By measuring SERS signals from colloidal solutions containing the same concentration of analyte but diluted in different fashions, it is shown that the method by which samples are diluted has a dramatic effect on interpretation of SERS spectra with further implications for single molecule SERS experiments. The results in Chapter 4 have profound impacts on the work in the other chapters.

Chapter 5 introduces the concepts, experimental and theoretical, surrounding the use of the novel integrating sphere setup developed for absorption measurements of dyes on metallic nanoparticles. The underlying equations governing the working principles of integrating spheres are presented. These are then applied to the case of sample contained within the sphere cavity and the calibration procedure for measuring absorption within the sphere is developed.

Chapter 6 presents arguably the most interesting results of the thesis. For the first time, the optical absorption spectra of commonly used dye molecules adsorbed to silver nanospheres in solution are measured, using the CMCIS 
setup described in Chapter 5, crucially at sub-monolayer dye coverages. Mietheory calculations are shown to accurately reproduce the observed changes in absorption spectra. Modifications of the dye absorption spectra upon adsorption are then discussed in the context of chemical modifications in SERS, through careful wavelength dependent SERS measurements of Crystal Violet.

\subsection{Scientific Outputs}

Much of the work in this thesis has been the subject of two publications of which I am the first author (see List of Publications). The work in Chapter 4 was published in the Journal of the American Chemical Society in 2014 and the work in Chapter 5 and 6 was published in Nature Photonics in 2016. A 3rd publication was accepted in Physical Chemistry Chemical Physics in 2015 but the details of this work are not discussed in this thesis.

There are many instances throughout this thesis of extensions to the published works that were not included in the final articles. In particular the COMSOL modelling section of Chapter 4 is extended and the SERS REP data for Crystal Violet were only alluded to in the original Nature Photonics paper. Also there are a number of graphs that have been reproduced from the articles but plotted in MATLAB and as such may look slightly different to those in the publications, yet no modification to the data itself was done. 


\section{Chapter 2}

\section{Theoretical Background}

This chapter introduces the most relevant theoretical foundations governing the experimental work presented in later chapters. The work carried out in this thesis spans the broad fields of molecular spectroscopy, Raman scattering, electromagnetism and plasmonics. As such, many of the concepts presented have been studied in far greater detail elsewhere and only the details most pertinent to this thesis are discussed below. For detailed description of molecular spectroscopy, the Raman effect and Surface Enhanced Spectroscopies, the reader is referred to the many great books on these topics [16,54-56]. The Chapter is split into two main sections; the first presents the main ideas of Molecular Spectroscopy with a focus on Raman scattering, while the second introduces the underlying principles of Surface Enhanced Spectroscopies and the origin of the interesting optical properties of noble metals.

\subsection{Molecular Spectroscopy}

In molecular spectroscopy, the interaction between light and molecules is used to obtain information about the chemical structure of molecules. A wealth of information can be extracted about the nature of the molecules being studied by studying this interaction, from the energy levels of electronic transitions (UV-Vis spectroscopy), the lifetimes of excited states (ultrafast fluorescence spectroscopy), all the way down to the coupling between electronic and vibrational states (resonance Raman spectroscopy) and complex non-linear optical 
processes (e.g. Hyper-Raman scattering and second-harmonic generation). In the context of this thesis, the main interest is in using optical spectroscopy to understand the electronic and vibrational transitions that occur in dye molecules when excited with electromagnetic radiation. As such this section gives a brief overview of the related spectroscopic concepts, with particular focus on Raman spectroscopy.

\subsubsection{Energy Levels of Molecules}

Most of the optical properties of atoms and molecules can be understood by considering the energy levels of such systems. The formulations of quantum mechanics describe the behaviour of an atom, or a many atom system (i.e. a molecule) in terms of distinct energy levels. These energy levels can be electronic (due to the energy of the electrons in the atom), vibrational (vibrations of the nuclei about the bonds in the atom) and ro-vibrational (rotational and/or vibrational motion of the nuclei) in nature.

These levels are depicted (neglecting rotational levels, that are not relevant here) in Figure 2.1, for a simplified model of a molecule consisting of two (electronic) levels, the ground state (denoted $S_{0}$ ) with energy $E_{0}$ and an excited state (denoted $S_{1}$ ) with energy $E_{1}$. Each electronic state is made up of a series of sub-states that are the allowed vibrational states associated with the vibration of the nuclei about thier equlibrium position for that particular electronic configuration. Both electronic and vibrational levels are drawn as solid lines to represent thier quantization into distinct energy levels. This simplified description allows a straighforward understanding of the transitions allowed when a molecule interacts with light. 


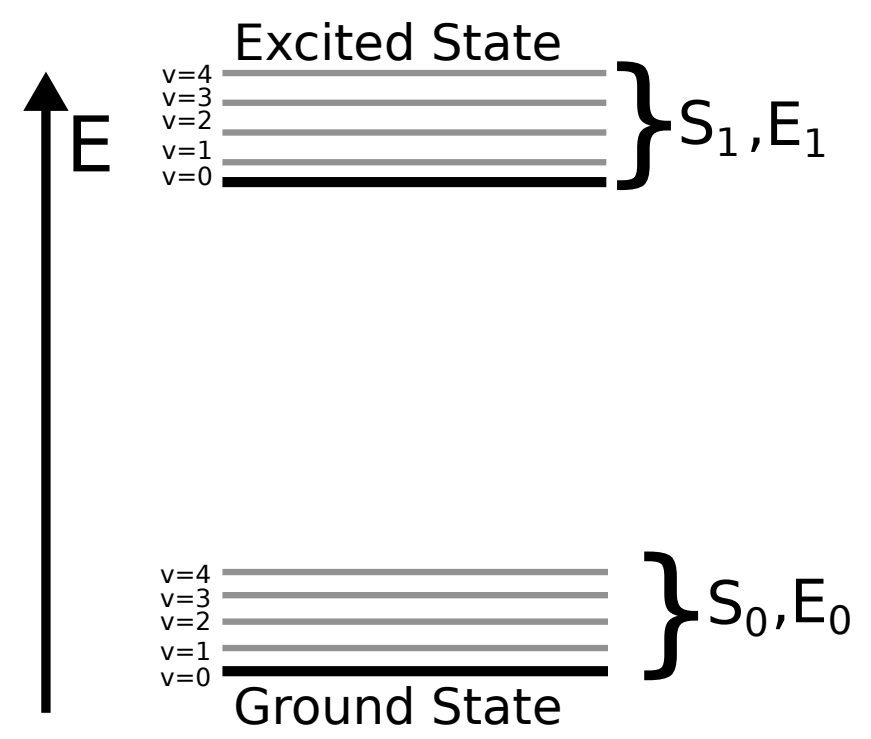

Figure 2.1: Electronic and vibrational levels of a molecule represented by an ideal two level system.

\subsubsection{Optical Transitions in Molecules}

When an atom (or molecule) interacts with electromagnetic radiation, the molecule can undergo a range of transitions that are determined by both the structure of the molecular energy levels and the energy of the incident radiation. The types of processes that can occur, for the two-level system, are depicted in Figure 2.2, known as a Jablonski diagram. The simplest process to consider is the absorption of energy by the molecule (Figure 2.2 (a)). If a photon of incident energy $E_{\text {In }}$ interacts with the molecule consisting of two electronic states, the photon can be absorbed by the molecule and induce a transition from the ground state, $E_{0}$, to the excited state $E_{1}$, with the molecule now being in an excited energy state with energy level $E_{1}=E_{0}+E_{\text {In }}$. Formally the probability for the transition to occur between the two states $E_{0}$ and $E_{1}$ is determined quantum mechanically by the coupling strength between the initial and final state wavefunctions, given by Fermi's Golden Rule, but for the treatment here it is sufficient to say that dipole allowed transitions can promote electrons from lower energy states to higher energy ones by the absoprtion of a photon. The reason the initial and final states are denoted $S_{0}$ and $S_{1}$ is that they are considered singlet states, where the total spin number of the electrons in each level is zero. Only transitions between states of the same total spin can be excited by a photon, so the transition to an excited 
state, for example the triplet, $T_{1}$ in (d), that has a different spin number than the ground state, is forbidden whereas the $S_{0} \rightarrow S_{1}$ is allowed [57].

(a)

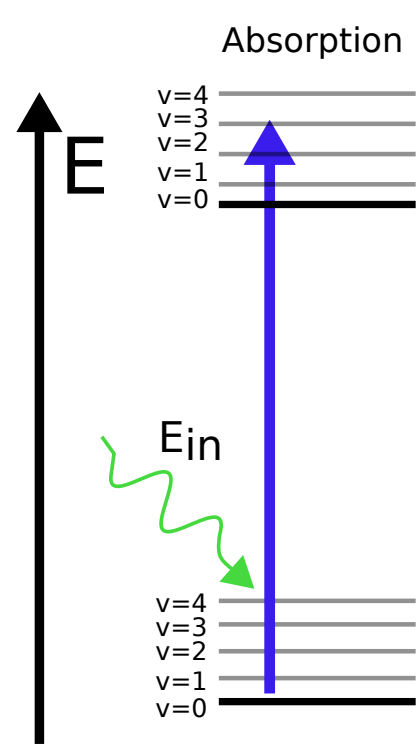

(b)

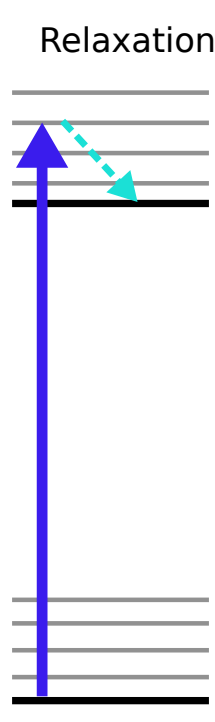

(c)

Emission (Fluorescence)

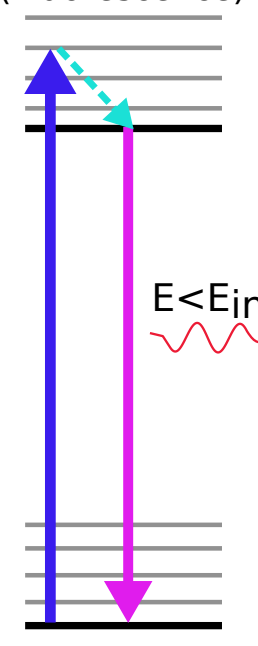

(d)

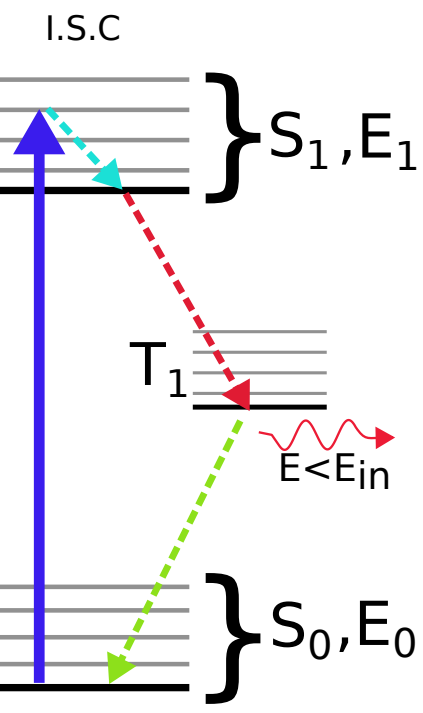

Figure 2.2: Jablonski diagram depicting the typical processes that a molecule can undergo when a photon is absorbed.

Figure 2.2 the shows the processes the molecule can then undergo after being excited to $E_{1}$ before decaying back to the ground state; because the electronic states of the molecule are made up of a sub-structure of vibrational energies, the incident photon can have energy higher than $E_{1}-E_{0}$, and thus the molecule can be in a higher vibrataional (excited) state, $v_{1}=0,1,2, \ldots k$ than it was in the ground state (Figure 2.2 (b). From this higher excited vibrational state the molecule can non-radiatively relax down to the lowest (excited) vibrational state, $v_{1}=0$. This relaxation typically occurs in the picosecond timescale, after which the molecule can decay back to the ground (electronic) state, $S_{0}$, through two processes; (1) the radiative emission of a photon with energy $E_{\text {Out }}$ less than that of the incident photon (Figure 2.2 (c)) or (2) by relaxation to a triplet state, $T_{1}$, whereby the molecule can either photobleach through interaction with its enviroment or emit a photon at a much later stage. Step (c) is the general description of fluorescence, while step (d) is known as inter-system crossing (ISC) and is the origin of phosphorescence. 
Despite this simplified treatment, it can now be understood that the absorption of light by a molecule can give detailed information about the electronic and vibrational states of the molecule. For real molecules, the system is significantly more complicated than a two-level system but the general principles are the same. The main difference between the ideal system presented here and a real molecule is the shape of the absorption spectra; for the two-level system excited by a white light source, a series of sharp absorption and emission bands would be observed, each corresponding to the various electronicvibrational transitions between the ground and excited energy levels. In a real molecule, the number of electronic states is much larger than two; the vibrational sub-structure associated with each electronic state then causes the absorption spectrum to be "smeared out" and the energy levels constitute more of a continuum of states rather than the discrete ones observed for the ideal system. As such, for large molecules like those used in this thesis, the vibrational sub-structure is often not visible and the absorption spectrum will exhibit a broad, continuous lineshape that represents the sum of all possible electronic-vibrational transitions [57].

\subsubsection{Optical Transitions in Dye Molecules}

The arguments above for an arbitrary set of energy levels can be extended to understand the optical properties of organic dye molecules, of which this thesis is mainly concerned. In a molecule with many atoms, the energy levels are determined by how electrons fill the molecular orbitals of the molecule, the highest energy of which is called the HOMO (highest occupied molecular orbital) and corresponds to the $S_{0}$ state as depicted before. The (unfilled) energy level directly above the HOMO level is known as the LUMO (lowest unoccupied molecular orbital) corresponding to the $S_{1}$ state. Since the electrons in the HOMO are those furthest away from the nuclei, they are weakly bound and can be easily excited into a higher state. The LUMO is the next lowest lying orbital available for these electrons to be excited into. The energy difference, $\Delta E$ between the HOMO and LUMO of a molecule will then determine the energy, or wavelength, at which light will be absorbed to promote electrons from the HOMO to the LUMO. This is depicted in Figure 2.3. 


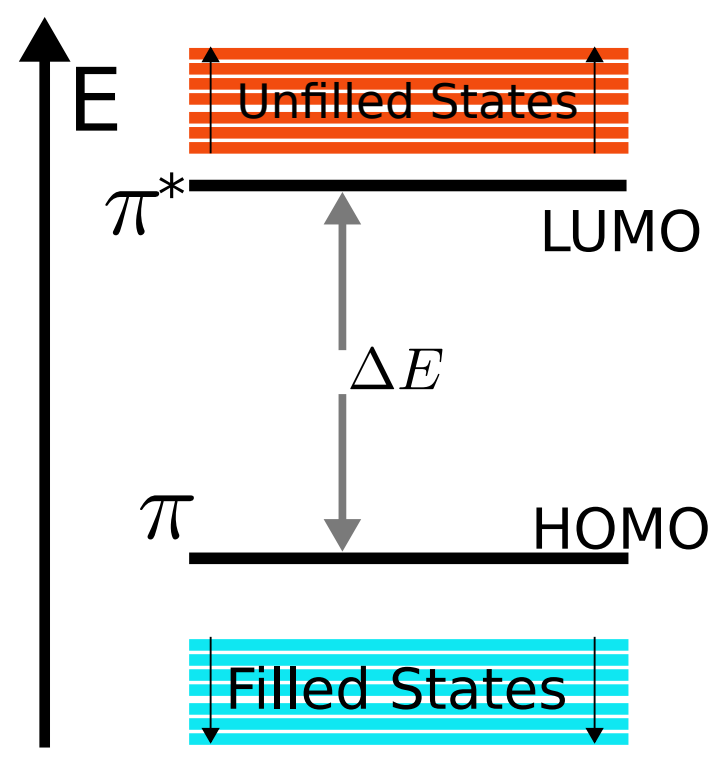

Figure 2.3: Depiction of the energy levels for a typical molecule. A typical $\pi \rightarrow \pi^{*}$ transition is shown between the HOMO and LUMO levels.

The energy, or wavelength, at which the HOMO-LUMO transition occurs depends on the type of orbitals that make up the HOMO and LUMO states. In $\sigma$ orbitals, the electrons are more tightly bound to the nuclei than in $\pi$ orbitals, and thus require more energy (lower wavelegnth) to promote an electron to a higher orbital than for $\pi$ orbitals. In molecules like aromatic dyes, there exist large conjugated systems of $\pi$ orbitals, where the electrons are spread out across the molecule, or delocalised, reducing the energy required to promote electrons from the HOMO to the LUMO. The larger the conjugated system, the easier it is to excite these $\pi$ electrons and thus the absorption wavelength is pushed into the visible region. The HOMO for these molecules will be a $\pi$ orbital and the strongest transition in the visible region will typically be to a so-called $\pi \rightarrow \pi^{*}$ transition.

As an example, the UV-Vis spectrum of Rhodamine $6 \mathrm{G}$, a typical organic dye with a large conjugated system is shown below in Figure 2.4. These dyes tend to posess two main absorption bands, an $S_{0} \rightarrow S_{1}$ and a higher energy $S_{0} \rightarrow S_{2}$ transition [40]. In the context of SERS, it is only the lower energy $S_{0} \rightarrow S_{1}$ transition that is of interest. This excitation occurs at $527 \mathrm{~nm}$ for RH6G in water and corresponds to the HOMO-LUMO gap between the largely de-localised $\pi$ orbitals of the xanthene ring. The transition dipole moment for the transition, $\mu_{D}$, is oriented parallel to this xanthene ring as shown, and 
has been confirmed with TDDFT calculations [40]. The dipole moment of the molecule, in particular its orientation, plays an important role in Surface Enhanced Spectroscopy and will be used in Chapter 6 to understand the process of surface enhanced absorption.
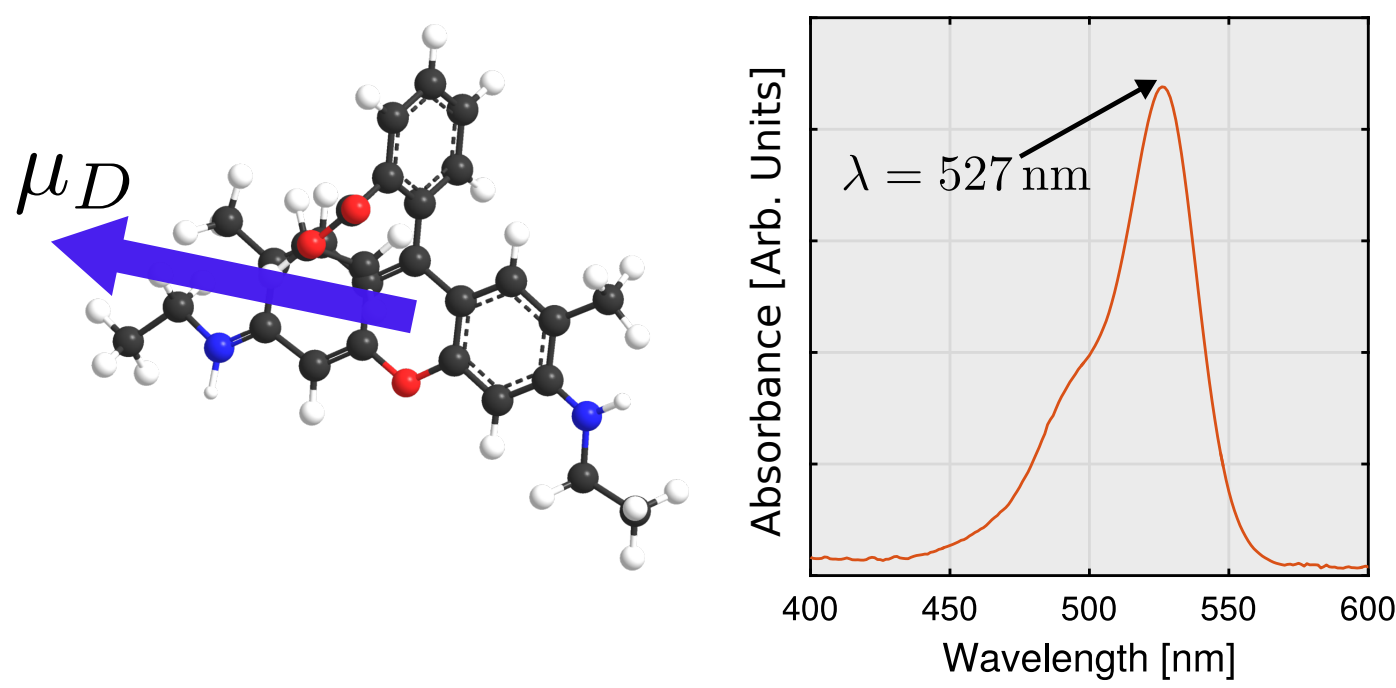

Figure 2.4: Chemical structure of RH6G along with its absorption spectrum in water. The blue arrow on the left indicates the orientation of the dipole moment, $\mu_{D}$, associated with the main $S_{0} \rightarrow S_{1}$ transition that corresponds to the absorption peak at $527 \mathrm{~nm}$. The dipole moment is oriented along the main xanthene backbone of the molecule.

Much of the terminology presented up to now will be used in the rest of the thesis to dicuss the processes that occur when molecules interact with light. The treatment is by no means exhaustive but is sufficient for the understanding of the topics discussed throughout the following chapters. While the processes up to now have involved absorption of light by a molecule, the focus is now switched to another possible light-molecule interaction, scattering. 


\subsection{Raman Scattering}

When light interacts with a molecule, there is a probability that the light can be scattered, i.e. absorbed and instantaneously re-emitted. The light can be scattered elastically, with the same frequency as the incoming light, known as Rayleigh scattering, or inelastically where the scattered frequency is different to the incoming light. The latter process is known as Raman scattering. First observed experimentally in 1928 by C.V Raman [58] and predicted 5 years earlier [59], Raman scattering arises due to the interaction of light with the vibrations of molecules. The three types of scattering processes possible are depicted in the Jablonski diagram in Figure 2.5 for an incoming photon of energy $E_{L}$.

- The first process (Figure 2.5 (a)), Rayliegh scattering, occurs where the scattered photon has the same energy as the incoming photon and there is no change in the energy level of the molecule before and after the scattering process occurs. Thus the process is elastic and there is no exchange in energy between the photon and the molecule.

- In the second process (Figure 2.5 (b)), the molecule absorbs some of the incoming photon energy and is left in a vibrationally excited stated (but with no change in the electronic state). In this case, the energy of the emitted photon is less than the incoming photon, $E_{S}=E_{L}-\hbar \omega_{\nu}$, where $\hbar \omega_{\nu}$ is the energy of the vibrational transition. This process is known as Stokes scattering.

- If the molecule is in an excited vibrational state $(\nu=1)$ upon interaction with the incoming photon (Figure 2.5 (c)), it can impart energy to the scattered photon by relaxation to the ground vibrational state $(\nu=1)$. The energy of the scattered photon is then greater than the incoming one, by $E_{S}=E_{L}+\hbar \omega_{\nu}$. This process is known as anti-Stokes scattering and typically less intense than its Stokes counterpart because the molecule needs to be in a vibrationally excited state, the population of which is thermally related to the Boltzmann temperature. Thus anti-Stokes scattering scattering intensity will be less than Stokes scattering by a factor $\exp \left(-\hbar \omega_{\nu} /\left(k_{B} T\right)\right)$, where $T$ is the temperature of the molecule. 


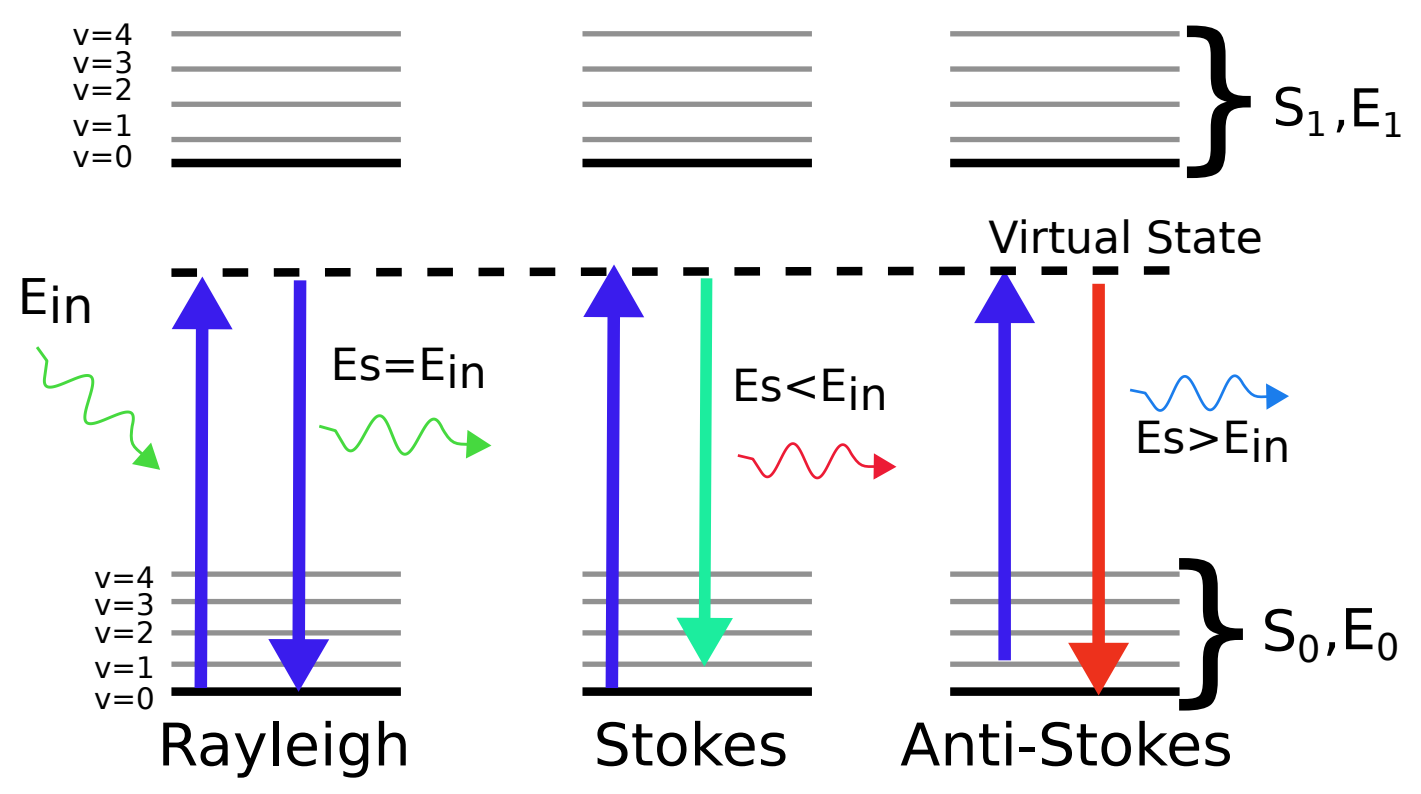

Figure 2.5: Jablonski diagram depicting (a) Rayleigh, (b) Stokes and (c) anti-Stokes Raman scattering.

The use of virtual levels in the diagrams of Figure 2.5 are to give a pictorial view of the scattering process while omitting many of the finer details governing the process. Because the scattering process is instantaneous, the molecule in reality spends no time in an excited state; as such this virtual state is considered an intermediate state of the molecule required for the scattering to occur and provides a qualitative understanding of the process. The concept of the virtual state orginates from the quantum mechanical treatment of the Raman process, where in second order perturbation theory the oscillating field of the incident photon perturbs the electron cloud for a very short period [56]. A full quantum mechanical treatment of the process can be found in Long's book [54] and the description here is limited to that presented in Figure 2.5 which is sufficient for understanding the most important aspects of the Raman effect.

\subsubsection{The Classical Description of Raman Scattering}

Classically, Raman scattering arises because of the change in the polarisability of a molecule due to the dipole induced in the molecule by an incident electric field. The linear optical polarisability tensor [16], $\hat{\alpha}_{L}\left(\omega_{L}\right)$ of a molecule 
relates the induced dipole moment, $\underline{\mathbf{p}}\left(\omega_{L}\right)$, to the incident electric field, $\underline{\mathbf{E}}\left(\omega_{L}\right)$, oscillating at frequency $\omega_{L}$, by:

$$
\underline{\mathbf{p}}\left(\omega_{L}\right)=\hat{\alpha}_{L}\left(\omega_{L}\right) \cdot \underline{\mathbf{E}}\left(\omega_{L}\right)
$$

where the dipole and the electric field are both written in complex notation. From this description, the field induces a dipole in the molecule oscillating at the same frequency as the field, and the dipole response is linear, as characterised by $\hat{\alpha}_{L}(\omega)$. In the case of Raman scattering, a dipole is also induced in the molecule but now oscillates at a frequency $\omega_{R}$, where for Stokes scattering $\omega_{R}=\omega_{L}-\omega_{\nu}$. In the same vain as before, the Raman polarisability tensor $\hat{\alpha}_{L}\left(\omega_{L}, \omega_{\nu}\right)$ is now introduced to characterise the response of the molecule to the field for a given mode at frequency $\omega_{\nu}$. This then leads to the definition of the induced Raman dipole that oscillates (and thus radiates) at $\omega_{R}$ :

$$
\underline{\mathbf{p}}_{R}\left(\omega_{R}\right)=\hat{\alpha}_{L}\left(\omega_{L}, \omega_{\nu}\right) \cdot \underline{\mathbf{E}}\left(\omega_{L}\right)
$$

While above approach is a phenomenological treatment and gives no indication of the physical origin of the Raman polarisability tensor for a given molecule, the idea of a Raman dipole as being induced by the incident field is sufficient for understanding many of the following processes relevant in the thesis, especially in the context of enhanced Raman scattering at a metallic surface.

\subsubsection{The Raman Spectrum}

In practice, Raman spectroscopy is performed by exciting a sample with a laser and detecting the scattered light with a spectrometer. What is measured by the spectrometer is the energy of the scattered light, which is most often expressed as the Raman shift, $\bar{\nu}_{j}$. This is usually given in wavenumbers $\left[\mathrm{cm}^{-1}\right]$ and is the energy difference between the incident light, $E_{L}$ and the scatterd light, i.e. the energy of the $j$ th vibrational mode. For a Stokes mode this is: 


$$
\Delta E_{R}=E_{L}-E_{S}
$$

A typical Raman shift will then be shown as intensity versus Raman shift [56], where the laser line will be at a Raman shift of $0 \mathrm{~cm}^{-1}$. The Stokes lines will be positioned on the positive Raman shift axis and the anti-Stokes on the negative side. Figure 2.6 shows a typical Raman spectrum obtained of 2-bromo-2-methylpropane (2B2MP) excited with a $633 \mathrm{~nm}$ laser. Each peak the corresponds to scattering by a particular vibrational mode of the molecule. In this thesis, only Stokes scattering is considered.

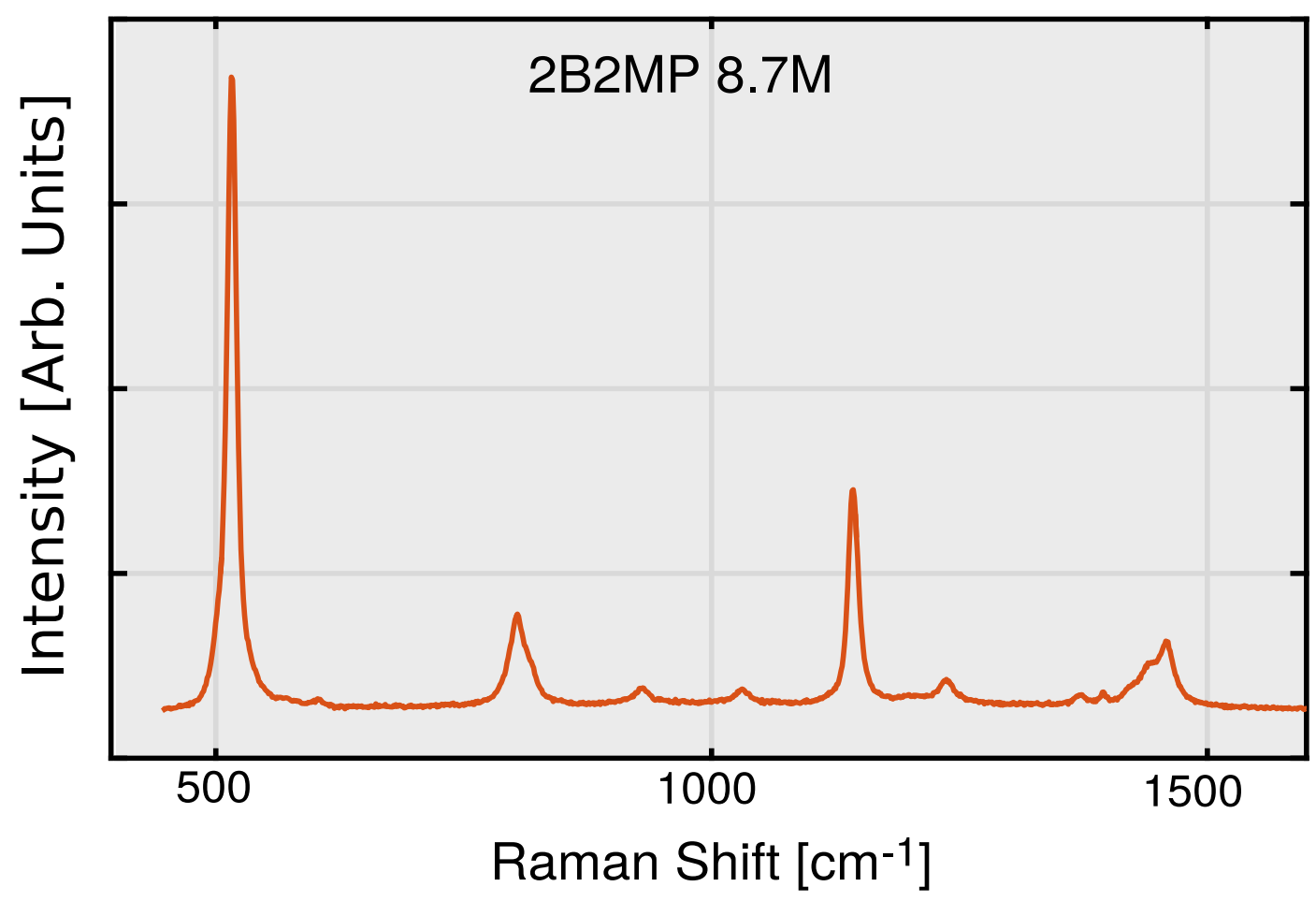

Figure 2.6: Raman spectrum of 2B2MP taken at $633 \mathrm{~nm}$.

\subsubsection{The Raman Cross Section}

A central parameter used throughout this thesis is the Raman cross-section that essentially characterises the optical strength of a given Raman mode. Like any cross section, the Raman cross section, $\sigma_{R}$, describes the probability of an incoming photon being Raman scattered; a cross section of $10^{-2}$ implies that for every 100 photons, only one will undergo Raman scattering. Physically, 
the cross section of a process describes the ratio of power produced by the process, $P[\mathrm{~W}]$ compared to the incident power density, $S_{I n}\left[\mathrm{~W} \mathrm{~m}^{-2}\right]$,

$$
\sigma=\frac{P}{S_{I n}}
$$

In practice, $\sigma_{R}$ is almost impossible to measure because it is the cross section for scattering in all directions. To measure this, one would have to collect the scattered light from the full $4 \pi$ steradians, which is not practical with standard spectrometers. In reality, the spectrometer configuration will be such that light is only collected over a small solid angle, $\mathrm{d} \Omega\left[\mathrm{sr}^{-1}\right]$. As such, a more pratical metric for the Raman cross-section, the differential Raman cross-section [16] be defined as:

$$
\frac{\mathrm{d} \sigma_{R}}{\mathrm{~d} \Omega}\left[\mathrm{cm}^{2} \mathrm{sr}^{-1}\right]=\frac{\mathrm{d} P_{R} / \mathrm{d} \Omega}{S_{I n}}
$$

In practice, the differential cross section of a given Raman mode can be measured experimentally by comparing the Raman peak intensity to the intensity of a reference molecule, measured under identical conditions, whose crosssection is known. Normalising for concentration differences, this gives [16]:

$$
\left(\frac{\mathrm{d} \sigma_{R}}{\mathrm{~d} \Omega}\right)_{\text {Sample }}=\left(\frac{\mathrm{d} \sigma_{R}}{\mathrm{~d} \Omega}\right)_{\text {Ref }} \frac{I_{\text {Sample }}}{I_{\text {Ref }}} \frac{c_{\text {Ref }}}{c_{\text {Sample }}}
$$

The differential Raman cross-section is central to this thesis and is mentioned many times, simply as the "Raman cross-section". It is directly relevant to SERS, being the parameter that is used to estimate by how much the Raman signal of a molecule is enhanced. Finally, typical Raman cross sections for non-resonant molecules are of the order of $10^{-30} \mathrm{~cm}^{2} \mathrm{sr}^{-1}$, which when compared with other optical processes such as fluorescence, whose cross-sections are of the order of $10^{-16} \mathrm{~cm}^{2} \mathrm{sr}^{-1}$, highlights the extremely weak nature of the Raman effect. 


\subsubsection{Resonance Raman Scattering}

In the above treatment of Raman scattering, the process is depicted such that the energy of the incident photon is much lower than the energy of any electronic transitions of the molecule. As such, the virtual state lies somewhere between the ground and excited electronic states, $E_{0}$ and $E_{1}$.

The quantum mechanical treatment developed by Plazeck is required to understand the underlying mechanisms of Resonance Raman scattering and is particularly cumbersome to derive. A full treatment of the problem is given in [54]. However the main ingredients relevant to SERS are captured by considering the transition probability, between an initial state $\langle i|$ and a final state $|f\rangle$ of the molecule, due to the (time-dependent) perturbation produced by an incident electric field on the wavefunctions of the initial and final states. It can be shown [54] that the transition polarisability, $\alpha_{k l}$ for the Stokes and anti-Stokes scattering of a given Raman mode, is given by:

$$
\alpha_{k l}=\frac{1}{\hbar} \sum_{r \neq i, f}\left\{\frac{\left\langle f\left|\hat{p}_{k}\right| r\right\rangle\left\langle r\left|\hat{p}_{l}\right| i\right\rangle}{\omega_{r i}-\omega_{L}-\omega_{1}-i \Gamma_{r}}+\frac{\left\langle f\left|\hat{p}_{l}\right| r\right\rangle\left\langle r\left|\hat{p}_{k}\right| i\right\rangle}{\omega_{r f}+\omega_{L}+\omega_{1}+i \Gamma_{r}}\right\} .
$$

Here $p_{k}$ and $p_{l}$ are the (quantum mechanical) dipole moment operators for the given transitions, the terms $\left(\omega_{r}-\omega_{i}\right)$ and $\left(\omega_{r}-\omega_{f}\right)$ represent the frequency of the scattered photon and $\omega_{L}$ that of the incident photon. Thus the left terms in the curly brackets represents Stokes scattering and the right terms anti-Stokes. $\Gamma_{r}$ is the half-width of the the level (in $\operatorname{rads}^{-1}$ ) which is related to the lifetime of the state. Finally the $\langle r|$ represents the virtual state, and the sum indicates all possible states (except for the initial and final states) are included in the summation [54].

Without dwelling on the details of Equation 2.7, what is clear is that when the laser frequency, $\omega_{L}$ approaches that of a molecular (electronic) transition frequency, $\omega_{r}-\omega_{i}$, then the denominator in the left term will become small and the response will become large; i.e. a resonance condition is reached. This is equivalent of saying that the virtual state, $r$ coincides with a real absorption level in the molecule; this situation is then known as Resonance 
Raman Scattering (RRS). The corresponding intensity of Resonance Raman is often orders of magnitude larger than "regular", non-resonant Raman due to the large denominator term in Equation 2.7.

A typical example of the enhancement in Raman cross-sections under resonant conditions is seen for RH6G, whose differential Raman cross-section has been measured to be $2.3 \times 10^{-24} \mathrm{~cm}^{2} \mathrm{sr}^{-1}$ at $532 \mathrm{~nm}$ excitation [60]. This is 6 orders of magnitude larger than typical non-resonant cross-sections. Such large cross-sections exhibited by dye molecules in the visible region are the reason for their predominant use in SERS, where the problems associated with working at ultra-low analyte concentrations are mitigated by the intrinsically large Raman response under visible excitation. In Chapter 6, the relationship between excitation wavelength, absorption spectrum and the resonant Raman cross section of dye molecules on metallic surfaces is investigated and the terminology of this section is useful to keep in mind. 


\subsection{Surface Enhanced Spectroscopies}

The following sections introduce the concepts necessary for understanding the origin of the SERS effect. The treatment follows closely that in [16] and begins with a discussion of the optical properties of metals. This naturally leads to the concept of surface plasmons, that are essentially responsible for the amplified Raman signals from molecules adsorbed to metallic nanostructures.

\subsubsection{Optical Properties of Noble Metals}

To understand the origin of surface enhanced spectroscopic techniques using metallic nanostructures as the antenna for localisation of the incident radiation, it is necessary to first understand the optical properties of metals. This begins with the Lorentz model for the interaction of light with oscillating dipoles.

The optical properties of a material can be characterized by its dielectric function $\epsilon(\lambda)$, that describes the macroscopic optical properties of a material. The simplest way to describe this response is through the Lorentz model [16]. Here the electrons are treated as a classical harmonic oscillators with a natural frequency $\omega_{0}$ excited by an external oscillating field. The system is then considered a forced harmonic oscillator with damping provided by the restoring force of the nuclei on the electrons. In the case of metals, where the conduction electrons can be considered as being free to move, $\omega_{0}=0$, and the displacement of the electrons, $x$ can be described as a damped harmonic oscillator with no restoring force under an external electric field $\mathbf{E}$ at frequency $\omega$. The equation of motion is then

$$
m_{0} \frac{d^{2} x}{d t^{2}}+m_{0} \gamma_{0} \frac{d x}{d t}=-e \mathbf{E}
$$

where $\gamma_{0}$, the damping term, represents the rate of collisions of free electrons within the metal and $m_{0}$ is the mass of the electrons. Note the electric field is written as a complex field $\mathbf{E}=\operatorname{Re}\left(\underline{\mathbf{E}}_{\mathbf{0}} \exp ^{-i \omega t}\right)$ where $\underline{\mathbf{E}}_{\mathbf{0}}$ is the complex amplitude of the field [16]. 
This is known as the Drude free electron model for metals. The solutions are Equation 2.3.1 are of the form $\underline{\mathbf{x}}=R e\left(\underline{\mathbf{x}}_{\mathbf{0}} \exp (-\mathrm{i} \omega \mathrm{t})\right)$ again in complex notation, giving:

$$
\underline{\mathbf{x}}=-\frac{e}{m_{0}\left(\omega^{2}+i \gamma \omega\right)} \mathbf{E}_{\mathbf{0}}
$$

If there are $N$ free electrons in the material, then the total induced polarisation from the displacement of all electrons is $P=-N e x$ and using the fact that from Maxwell's equations, the electric displacement, $D=\epsilon_{0} \mathbf{E}+P$, it follows that the relative dielectric function is given by $[16,57]$ :

$$
\epsilon(\omega)=1-\frac{N e^{2}}{\epsilon_{0} m_{0}} \frac{1}{\omega^{2}+i \gamma \omega}
$$

If the optical response of bound electrons and positive ions are taken into account, the expression for the dielectric function of a metal can be written as $[16]$ :

$$
\epsilon(\omega)=\epsilon_{\infty}\left(1-\frac{\omega_{p}^{2}}{\omega^{2}+i \gamma_{0} \omega}\right)
$$

and $\epsilon_{\infty}$ represents the contribution of positive ions and bound electrons to the overall response. $\omega_{p}=\sqrt{n e^{2} / m \epsilon_{0} \epsilon_{\infty}}$ is known as the plasma frequency and is the natural oscillation frequency of the conduction electrons, analogous to the $\sqrt{\frac{k}{m}}$ term for the resonant frequency of a classical harmonic oscillator.

Finally by separating the dielectric function into its real and imaginary components:

$$
\operatorname{Re}(\epsilon(\omega))=\epsilon_{\infty}\left(1-\frac{\omega_{p}^{2}}{\omega^{2}+\gamma_{0}}\right) \quad \text { and } \quad \operatorname{Im}(\epsilon(\omega))=\frac{\epsilon_{\infty} \omega_{p}^{2} \gamma_{0}}{\omega\left(\omega^{2}+\gamma_{0}^{2}\right)}
$$



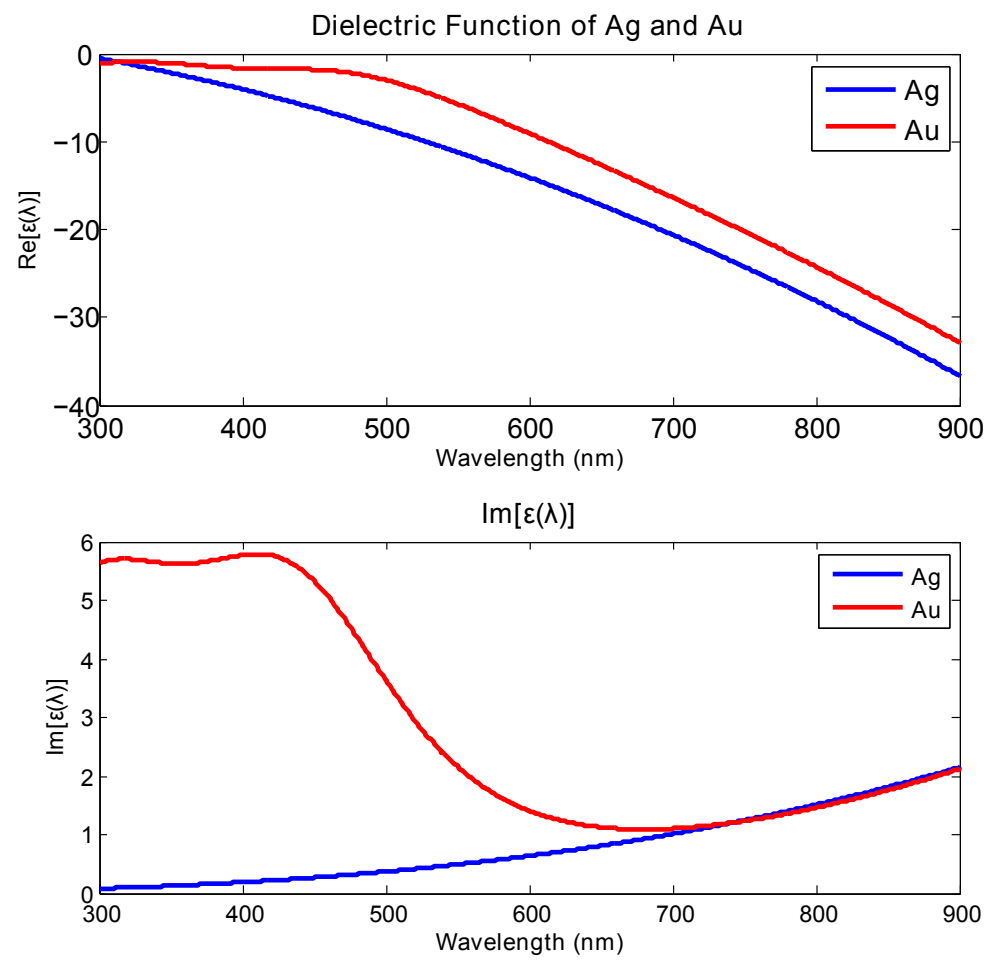

Figure 2.7: Dielectric functions of silver and gold obtained from the analytical model in Appendix E of [16].

Figure 2.7 shows the real and imaginary parts of $\epsilon(\lambda)$ for both Silver and Gold. These plots are based on analytical models (see Appendix E of Ref. [16]) that are in good agreement with experimental results $[61,62]$ and illustrate the optical properties of both metals as a function of wavelength. What is most notable, and whose importance will become apparent in the following section, is that for both metals, the real parts of the dielectric functions in the visible region, (where $\omega<\omega_{p}$ ) are negative and large while the imaginary part is small. It is essentially this feature of metals that gives rise to the interesting electromagnetic phenomena observed in the visible region, allowing effects such as SERS to exist. This point will become clearer in the next section. 


\subsubsection{Surface Plasmon Resonances - The Electrostatic approximation}

In this section the simplest case of a metallic sphere of radius $a$ embedded in a non absorbing medium with a dielectric constant $\epsilon_{M}$ in the presence of an external electric field is considered. This treatment, known as the Electrostatic Approximation (ESA) can be used to qualitatively demonstrate how the electric field on the surface of the sphere can be enhanced by satisfying the resonance condition of the system. The approach is valid in the limit that the radius of the particle is much less than the wavelength of the light creating the incident field, i.e. $a \ll \lambda$ where the electric field is essentially constant over the spatial dimensions of the sphere.

Figure 2.8 illustrates this simple model. The electrostatic solution to this well-known problem is described in detail in numerous textbooks [63] and involves solving the Laplace equation for the potential [64], $\nabla^{2} \phi=0$ from which the electric field can be calculated as the gradient of the potential, $\mathbf{E}=-\nabla \phi$. For the purposes here, the electric field on the surface of the sphere at point $\mathrm{A}$ is of interest.

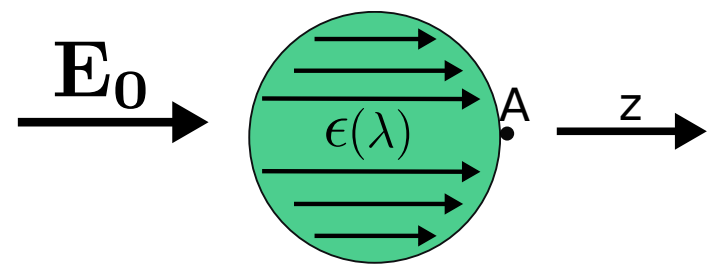

Figure 2.8: Metallic sphere of radius $a$ in the presence of a constant electric field $\mathbf{E}_{\mathbf{0}}$

Firstly, solving for the potential inside and outside the sphere and applying the appropriate boundary conditions, it can be shown [63] that the dipole induced inside the sphere is given by

$$
\mathbf{p}_{\text {ind }}=\frac{\epsilon(\lambda)-\epsilon_{M}}{\epsilon(\lambda)+2 \epsilon_{M}} r^{3} \mathbf{E}_{0} \cdot 4 \pi \epsilon_{0}
$$

where $\epsilon(\lambda)$ is the (complex) dielectric function of the sphere material and $\epsilon_{M}$ is the dielectric function of the embedding medium. It is immediately evident 
that a resonance condition, known as the dipolar localized surface plasmon resonance (LSPR), is reached when $\operatorname{Re}[\epsilon(\lambda)]=-2 \epsilon_{M}$. For normal dielectrics, where $\operatorname{Re}[\epsilon(\lambda)]$ is positive and $\operatorname{Im}[\epsilon(\lambda)]$ is large in the visible region, this resonance condition is never satisfied. For metals however, and in particular Ag and $\mathrm{Au}$, it is clear from Figure 2.7 that in the visible region it is possible to have $\operatorname{Re}[\epsilon(\lambda)]$ negative and $\operatorname{Im}[\epsilon(\lambda)]$ small. Thus the dielectric properties of metals allow the resonance condition of the sphere to be satisfied.

A few points on LSPRs are noted here. This result is central to the origin of the field enhancements in surface enhanced spectroscopies and illustrates why silver and gold have been the predominant materials used in plasmonics. LSPRs of nanostructures can generally be observed experimentally in the extinction spectrum and in the particular case of colloidal silver spheres used throughout this thesis, the LSPR is evidenced as a strong absorption peak in the 400 to $450 \mathrm{~nm}$ regime (see Chapter 3). Equation 2.13 also highlights the strong dependence of the LSPR position on the material properties. The dispersion of $\epsilon$ of the material imposes that for materials of different types, the maximum LSPR excitation will occur at different wavelengths of the incident light. As an example, the dipolar LSPR in for gold (in the ESA) occurs at $\sim 512 \mathrm{~nm}$ and for silver at $\sim 387 \mathrm{~nm}$. Thus the plasmonic properties can be tuned by the choice of material which has implications in applications where the excitation wavelength of the light is important.

It follows from the solution of the electrostatic problem that the field outside, $\mathbf{E}_{\text {out }}$, on the surface of the sphere assuming excitation along $z$ is just the sum of the incident field and the field of the induced dipole and is given by

$$
\mathbf{E}_{\text {out }}(r=a)=\mathbf{E}_{0}\left[\left(1-\beta_{S}\right) \mathbf{e}_{\mathbf{z}}+3 \beta_{S}\left(\mathbf{e}_{\mathbf{r}} \cdot \mathbf{e}_{\mathbf{z}}\right) \mathbf{e}_{\mathbf{r}}\right]
$$

where $\mathbf{e}_{\mathrm{r}}$ and $\mathbf{e}_{\mathrm{z}}$ are the surface normal and $z$-axis unit vectors respectively. Finally,

$$
\beta=\frac{\epsilon(\lambda)-\epsilon_{M}}{\epsilon(\lambda)+2 \epsilon_{M}}
$$

As can be seen the field outside also experiences a resonance at the same condition of $\operatorname{Re}[\epsilon(\lambda)]=-2 \epsilon_{M}$. This field is denoted the local field, $\mathbf{E}_{\mathrm{Loc}}$, and is the main component of interest for surface enhanced spectroscopies given 
that a molecule adsorbed to the sphere will be subjected to this field.

The Local Field Intensity Enhancement Factor (LFIEF) can now be introduced. The LFIEF [11] expresses the magnitude by which the intensity of the incoming field is enhanced at a point on the particle surface. Thus the LFIEF is formally defined as the ratio of the intensity of the field at position $\mathbf{r}$ to the intensity of the incident field:

$$
M_{\mathrm{Loc}}(\mathbf{r}, \lambda)=\frac{\left|\mathbf{E}_{\mathrm{Loc}}(\mathbf{r}, \lambda)\right|^{2}}{\left|\mathbf{E}_{\mathrm{Inc}}(\lambda)\right|^{2}}
$$

Using the expressions above and the analytical expression described in Appendix E of Ref. [16] for the dielectric function of silver, the wavelength dependence of $M_{\text {Loc }}$ for a silver sphere in water at point A can be plotted. Figure 2.9 shows that for the above electrostatic approximation, the LFIEF at point $\mathrm{A}$ on the sphere is predicted to be $\sim 4000$ for an excitation wavelength of $387 \mathrm{~nm}$.

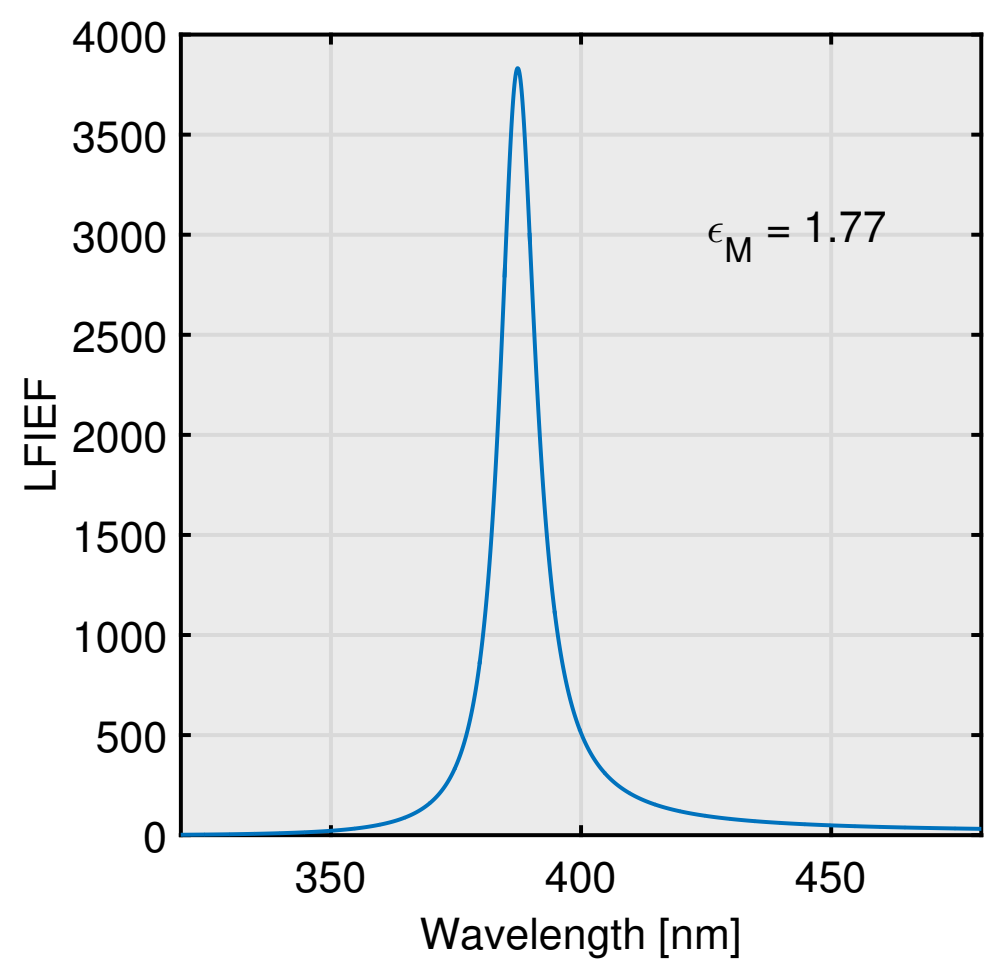

Figure 2.9: Electrostatic approximation of the LFIEF as a function of wavelength for a silver sphere in water. 
Finally, the local field can be separated into its components parallel and perpendicular to the surface given by:

$$
M_{\mathrm{Loc}}=M_{\mathrm{Loc}}^{\perp}+M_{\mathrm{Loc}}^{\|}
$$

In most cases only the total enhancement, $M_{\text {Loc }}$, is of interest but in certain contexts it is useful to know the value of the local field components. This will be relevant in Chapter 6 when discussing surface enhanced absorption of molecules at the metal surface. 


\subsubsection{The $E^{4}$ approximation in SERS}

The origin of the SERS effect can now be understood in the context of the enhanced local field at the surface of the nanoparticle due to the excitation of localised surface plasmon resonances. When the wavelength of incident light matches the resonance condition, the LSPR of the particle can be excited. For a molecule placed at, or very close to, the surface of the particle, the electric field felt by the molecule will be greatly enhanced with respect to the field it would experience in the absence of the sphere. It follows that the modified local field will induce a Raman dipole at frequency $\omega_{R}$ in the molecule proportional to the local field i.e.

$$
\mathbf{p}_{\mathbf{R}}=\alpha_{R} \mathbf{E}_{\mathrm{Loc}}\left(\omega_{L}\right)
$$

In the simplest approximation, ignoring any orientation or polarisation effects, the magnitude of the dipole induced will be increased by a factor given by $E_{\mathrm{Loc}}$ compared to the free dipole in the absence of the metal. In the absence of the metal, this dipole would then radiate into free space with an intensity proportional to $M_{\text {Loc }}$. This process can then be extended to find the overall factor by which the Raman signal radiated by the molecule is enhanced. By assuming that the re-emission branch of the Raman process is enhanced by the same amount as the local field, possibly the most important expression in SERS [16], namely the $|E|^{4}$ approximation is arrived at. Under the two assumptions, (1) that the excitation and emission branch of the Raman process are enhanced equally and $(2)$ the Stokes-shift for Raman mode is small ( $\left.\omega_{\text {Raman }} \approx \omega_{\text {laser }}\right)$ the $|E|^{4}$ approximations SERS EF for a single molecule, denoted SMEF, is given by

$$
\operatorname{SMEF}\left(\omega_{L}\right) \approx \frac{\left|E_{\mathrm{Loc}}\left(\mathbf{r}, \omega_{L}\right)\right|^{4}}{\left|E_{0}\right|^{4}}
$$

A number of details have been left out in the preceding derivation of the SERS Enhancement Factor. Firstly, the ESA described above is just that, an 
approximation. It fails to account for any higher order multipole excitations and would not provide correct predictions for the plasmon resonance of nonspherical objects (Chapter 6.2.1 Ref [16]), nor does it correctly account for the size dependence of the plasmon resonances. More advanced electromagnetic methods must be employed to predict the responses of such objects; Mie theory [65] must be used to fully account for the size dependence of LSPR and local fields for spherical objects and is treated briefly in Section 2.3.4. In the case of complicated nano-structures for which there exists no analytical solutoon, numerical electromagnetic methods ,not used in this thesis, must be employed such as finite-element methods (FEM), finite-difference timedomain (FDTD), boundary element method (BEM) and the surface integral equation (SIE) method.

Additionally, arriving at the $|E|^{4}$ approximation for the overall enhancement of the Raman signal requires a much more rigorous treatment of the underlying processes involved than the one presented here. The approximation finds its origin in what is known as the optical reciprocity theorem and its derivation is given in [66]. The approximation is in itself also a simplification of the situation. It fails to consider a number of other factors involved such as polarization and orientation effects. Nevertheless, both the ESA and the $|E|^{4}$ approximation provide a qualitative framework in which the processes governing the SERS effect can be understood. Throughout the rest of the thesis, the LFIEF and the SMEF will be referred to as $M_{\text {Loc }}$ and $F$, and more often the average values, $\left\langle M_{\mathrm{Loc}}\right\rangle$ and $\langle F\rangle$ will be used, which are the surface averages of the enhancement factors. These are more relevant experimentally especially when working with colloidal solutions.

\subsubsection{Mie Theory}

As mentioned in Section 2.3.2, the electrostatic approximation is only valid for small spheres, and fails to account for the size dependence of the surface plasmon resonance of the sphere. While the ESA is informative for understanding the general processes underlying LSPRs, many experimental settings will work with nanoparticles whose size is larger than the limit in which the ESA is valid. As such, it cannot be used to predict the plasmonic properties of these larger spheres. 
To study the LSP resonances of larger spheres, the full electromagnetic problem of scattering of electromagnetic waves by a spherical objects must be solved. Fortunately, there exist exact analytical solutions for this problem, and its formulation is better known as Mie-theory, named after its originator, Gustav Mie who first worked out the full solution in 1908. Since then, Mietheory has been used in a wide range of applications [67] far outside the realm of nanoparticles, and is applicable to scattering by spheres of arbitrary size. A more detailed treatment of Mie-theory is presented in Appendix A, while a full treatment can be found in References $[16,68]$.

The "simplicity" of the solution of scattering by a sphere lies in the symmetry of the problem, and the solution essentially consists of treating the incident and scattered fields as vector spherical harmonics (VSHs) that allow for multipolar expansions of the fields in spherical coordinates. Through the application of boundary conditions, the fields inside and outside can be solved for, allowing the optical response of the sphere to be determined that are dependent on the properties of the sphere. The equations are particularly complex because of the nature of the VSHs but there exist many numerical packages that quickly and accurately compute the response of spheres excited by electromagnetic waves. The package used in this thesis is the SPlaC code [69], written in Matlab. These codes compute the full solution of the problem and allow calculation of both the near and far-field properties of the sphere. In all cases here, it is assumed the sphere is subjected to plane-wave excitation only.

Without going into the details of the problem, in relation to SERS there are 5 major variables one wishes to solve for in Mie-theory that can be extremely helpful in interpreting experimental measurements. The three far-field properties are the extinction, scattering and absorption cross section, $\sigma_{\text {Ext }}, \sigma_{\text {Scat }}$ and $\sigma_{\mathrm{Abs}}$. The near field properties are the (surface averaged) LFIEF, $\left\langle M_{\text {Loc }}\right\rangle$ and the SERS enhancement factor, $\langle F\rangle$. In Figure 2.10 the wavelength dependence of these 5 parameters is plotted as calculated using the SPlaC codes for a $30 \mathrm{~nm}$ radius silver sphere embedded in a surrounding dielectric medium with $\epsilon_{M}=1.77$, i.e. water. The LSPR of the particle is clearly evident in the strong peak in the far-field spectra at $430 \mathrm{~nm}$. The (average) local field and (average) SERS EF also show the largest values at the wavelength corresponding to the LSPR again highlighting that the maximum field enhancements occur at the plasmon resonance wavelength (at least in the case of spheres). For the silver 
sphere, $\left\langle M_{\text {Loc }}\right\rangle$ is predicted to be a maximum of $\sim 75$ at the LSP resonance and the corresponding average SERS EF, $\langle F\rangle$ is predicted to be $\sim 5 \times 10^{3}$.

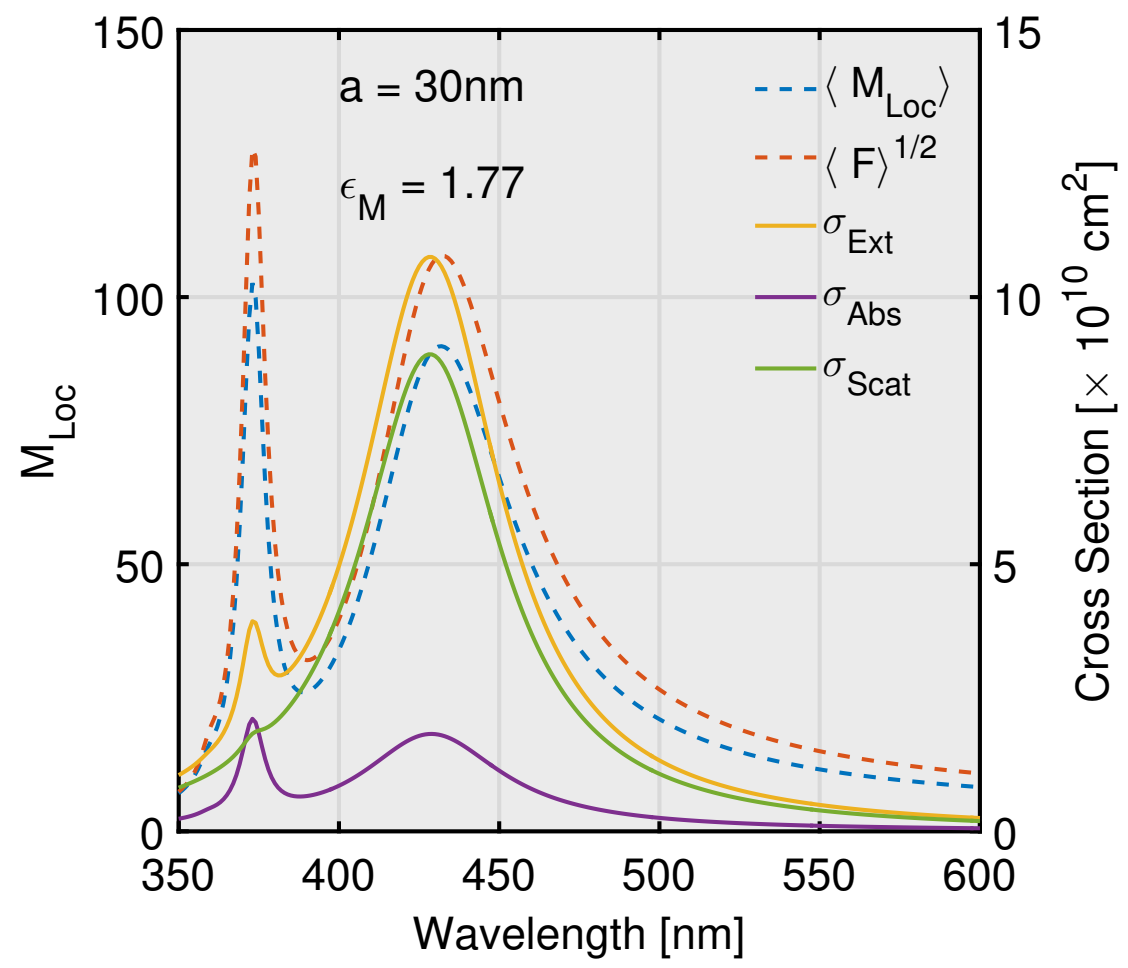

Figure 2.10: Predictions from Mie theory of the far field (extinction, scattering, absorption spectra) and local field ( $\left\langle M_{\text {Loc }}\right\rangle$ and $\left.\langle F\rangle\right)$ properties of a $30 \mathrm{~nm}$ radius silver sphere in water under plane wave excitation. Note that $\langle F\rangle$ is plotted as $\langle F\rangle^{1 / 2}$ for illustration.

These 5 parameters can be directly related to experimental observations for a typical SERS experiment. As will be shown in Chapter 3, the far-field properties can by used to compute the concentration of a solution of colloidal nanoparticles and also serve as a tool to understand the size dispersion and aggregation state of the colloids. The local field properties can be used to understand the magnitude of the observed SERS enhancements for analyte molecules placed on the surface of such colloids. This is of particular relevance to Chapter 4 , while knowledge of the predicted $\left\langle M_{\text {Loc }}\right\rangle$ for silver spheres is of crucial importance to interpreting the surface enhanced absorbance results in Chapter 6. As such, Mie-theory is a powerful tool that is utilised throughout this thesis to understand many aspects of the systems being studied.

Finally while Mie-theory constitutes the full solution to the electromagnetic scattering by spheres, in the case of plasmonics it does have some notable 
limitations for comparing predicted values to experimental observations. A few of these are listed below:

- The method is only valid for perfect spheres, whereas in reality, most nanoparticles synthesised by wet-chemistry methods, such as those shown in Chapter 3 are not perfectly spherical. Particles are often more polygonal in nature with sharp corners and irregular edges (See TEM images in Chapter 3). Mie-theory does not account for these morphologies. While not so important for the far-field properties (as seen later in Chapter 3 by the excellent agreement between theory and experiment for silver colloids), the near-field enhancements may be underestimated by not considering the sharp edges.

- The inevitable surface roughness of nanoparticles is not accounted for which could increase the local field at the surface in a similar way to the non-spherical geometrical effects.

Despite these shortcomings, Mie-theory correctly accounts for the majority of the effects creating the SERS enhancements and discrepancies with theory will be discussed on a case-by-case basic as they arise in later Chapters.

\subsubsection{Hot Spots in SERS Substrates}

Up to this point, the plasmon resonances and the SERS EFs obtainable by exciting them, has been limited to single spheres as the nanostructure providing the electromagnetic enhancement. Central however to the generation of the enormous enhancements often observed experimentally are the idea of "hot-spots", regions of highly localised electric fields, at the junctions between particles [39] or at the tips of sharp metallic particles [32,70], that greatly exceed those for spheres. The most canonical example of hot spots is the dimer of metallic spheres separated by distances of a few nanometers; in the gap region between both particles, the extreme localisation of the electric field can lead to maximum SERS enhancements of up to $10^{11}$ for molecules positioned within the hot-spot. These type of hot spots arise from the interaction between both spheres, creating new "coupled" or "gap-mode" LSP resonances that strongly 
confine the incident electric field within the particle junction, and are responsible for the enormous SERS enhancements that make SM-SERS possible.

The electromagnetic properties of SERS hot-spots can be understood by using generalised Mie-Theory (see Reference [16] Appendix H) for the didactic case of a dimer of silver spheres. Figure 2.11 highlights the main plasmonic properties of such a system relevant to SERS, specifically for the case of two $25 \mathrm{~nm}$ silver spheres separated by varying distances, $g$.

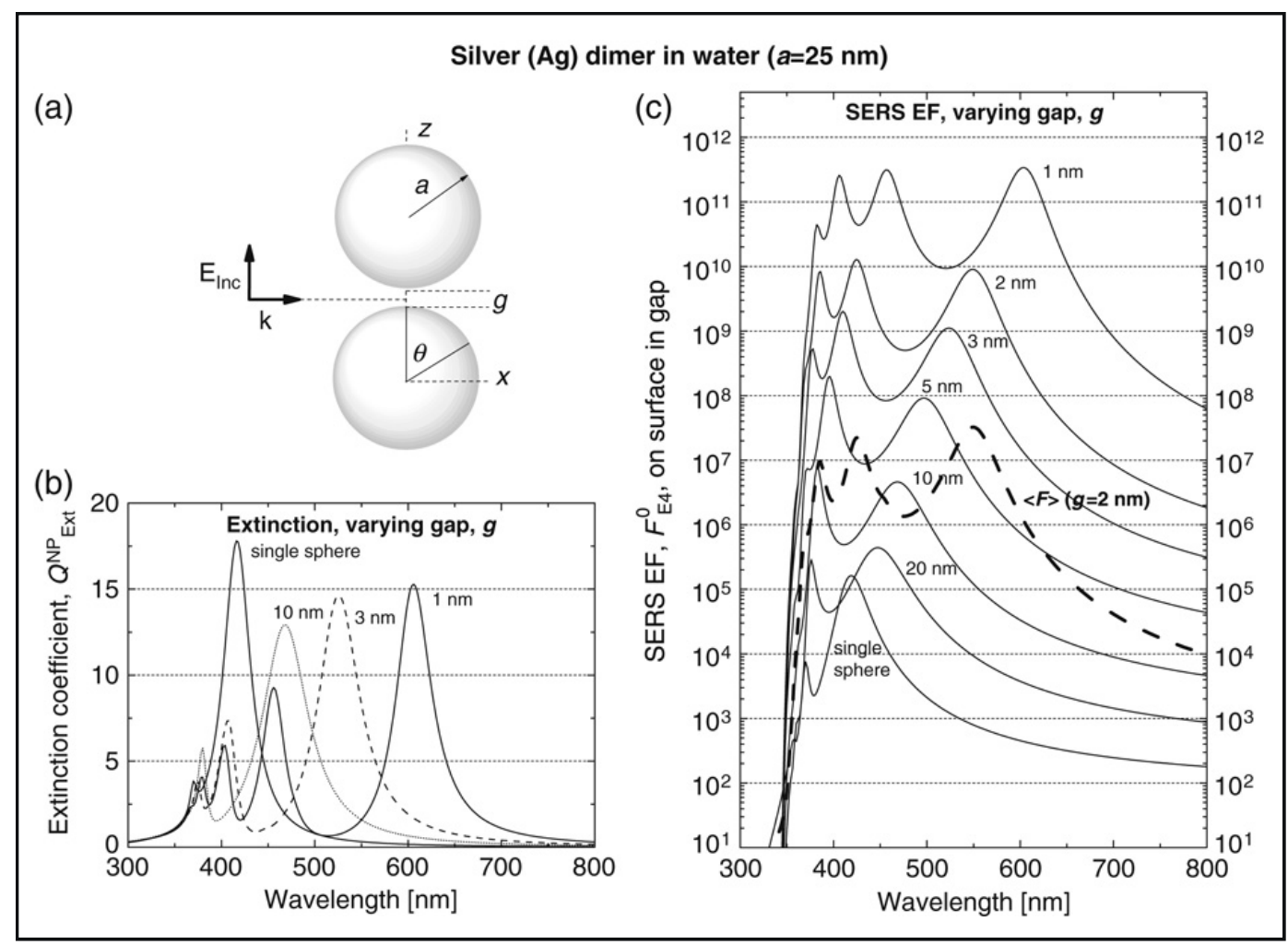

Figure 2.11: Predictions from Mie theory of the extinction spectra and SERS EFs of a dimer of $25 \mathrm{~nm}$ radius silver spheres with varying gap sized, $g$. Reproduced with permission from [16].

The first point of interest for the silver dimer is the predicted SERS EF; for a point above the surface of one of the spheres, inside the gap region, the EFs are large across the visible regions, ranging from $10^{6}$ for a $10 \mathrm{~nm}$ gap to a maximum of $10^{11}$ at $\sim 600 \mathrm{~nm}$ for a gap of $1 \mathrm{~nm}$. Thus molecules placed at this position will exhibit enormous Raman signals. Secondly, it is clear that the maximum EFs are extremely sensitive to the gap size; a change in gap size of only $1 \mathrm{~nm}$ the predicted maximum EF changes by an order of magnitude. This point crucially explains why the observed EFs in SERS experiments can exhibit 
large fluctuations; slight variations in substrate geometry (i.e. a solution of colloids in which there are dimers) across the sample can significantly affect the observed Raman signals. For example, in the same solution of colloids, two molecules residing within different dimer hot-spots, one with a gap of $1 \mathrm{~nm}$ and the other with a gap of $3 \mathrm{~nm}$ will have SERS signals that can differ by a factor of 100. Thirdly, the average SERS enhancements (shown for a $2 \mathrm{~nm}$ gap, dashed line) are predicted to be lower than the maximum EF but still can be 2 orders of magnitude higher than those for a sphere. Finally as the dimer gap is decreased, the main dipolar LSP wavelength redshifts from the LSP of a single sphere, as observed in the predicted extinction spectra, tracking roughly the same spectral shape of the local field. It follows that the far field properties can be used as a (rough) guide to the wavelength where the maximum EFs will occur [71].

The creation of hot-spots in SERS substrates is thus paramount to achieving high SERS enhancement factors. The simplest and most common method for achieving this by aggregation of a colloidal solution of nanospheres which is the preferred method in this thesis. This approach is simple way of producing a large number of highly active gap-mode hot-spots that are often sufficient for performing SM-SERS experiments. The drawback is the difficulty in controlling the gap size. As such these substrates often display a large variation of EFs due to varying gap sizes across the colloidal solution and many improved approaches in controlling the aggregation step have been studied [39]. The induced aggregation method will be addressed in more detail in Chapter 3. 


\section{Chapter 3}

\section{Experimental Details}

This chapter introduces the various experimental details and techniques governing the measurements related to Chapters 4, 5 and 6. The Raman spectrometer setup that was used for SERS measurements is described, and the historical context of colloidal solutions as SERS substrates is discussed. The types of colloids used are then presented along with the general scheme for preparing SERS samples and calculating average enhancement factors for the anlayte being studied. The spectroscopic and chemical properties of common SERS dyes are then addressed, with regards their electronic and Raman spectra and finally the exact procedures for calculating enhancement factors, both for average and single-molecule SERS measurements are outlined. This chapter should serve as a self-contained reference for the experimental work in the following Chapters.

\subsection{SERS Experimental Setup}

The experimental configuration for SERS measurements used in this thesis is depicted in Figure 3.1. The spectrometer used was a Jobin-Yvon LabRam microscope-based Raman spectrometer equipped with a liquid nitrogen cooled Symphony CCD detector and holographic notch filters for rejection of Rayleigh scattered laser light. The laser is delivered to the sample via a series of mirrors and focused into the sample by a microscope objective whose magnification is 


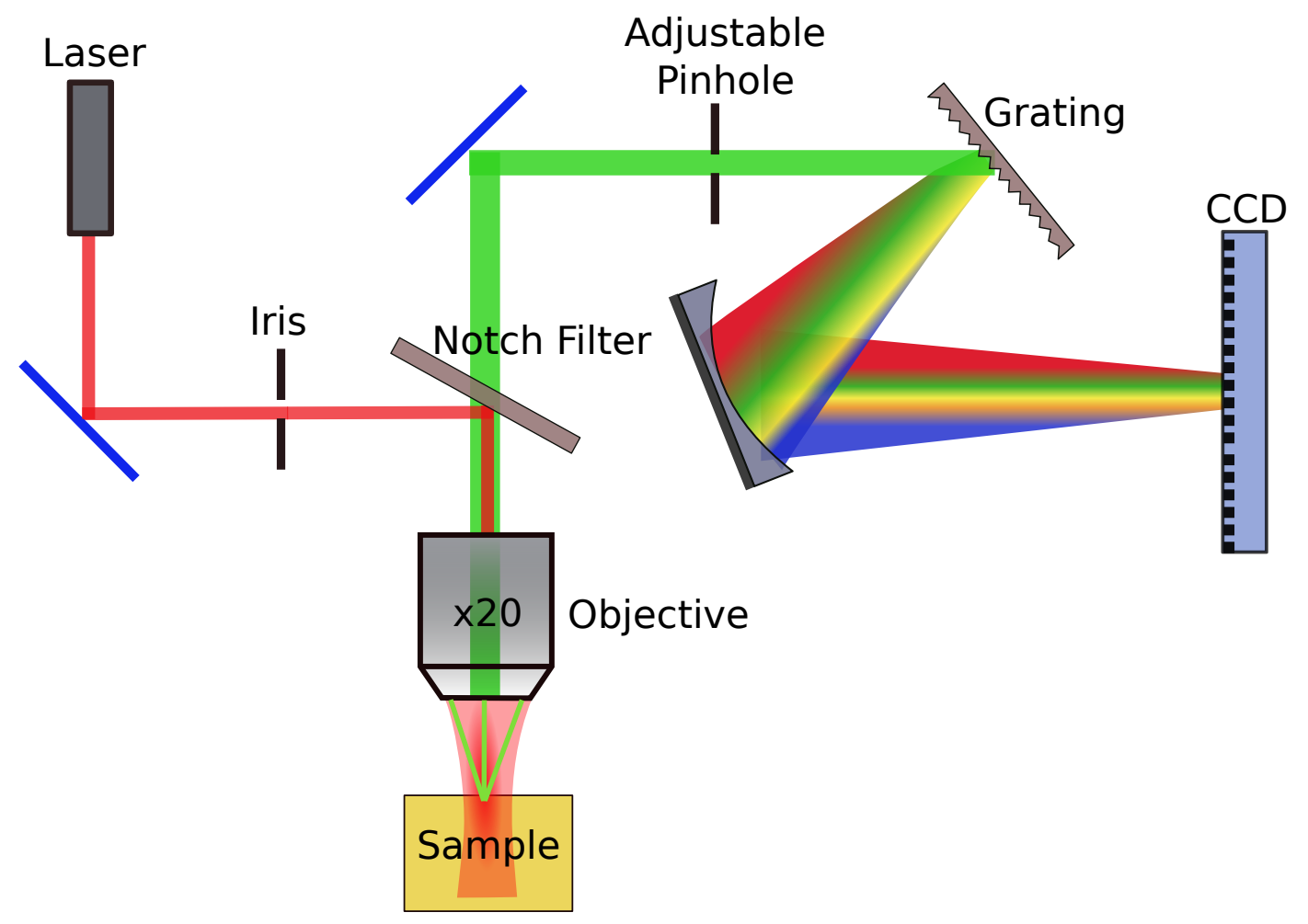

Figure 3.1: Optical schematic of the LabRam Raman microscope spectrometer.

chosen dependent on the experiment in question. The adjustable pinhole can be used to increase or decrease spectral resolution and reject stray light. The scattering volume for both the x20 and x100 objectives have been charaterised previously [12] using scan-over-edge and z-scan depth profiling methods. The $\mathrm{x} 100$ effective scattering volume was found to be $\approx 13 \mu \mathrm{m}^{3}$ that is used in SMEF calculations (as outlined in Chapter 2). Prior to acquisition of SERS spectra, the spectrometer is aligned and calibrated using the $520 \mathrm{~cm}^{-1}$ line of a silicon substrate. All spectra were acquired in the $20 \mathrm{kHz}$ readout mode of the CCD in the high-gain setting. 


\subsection{Colloidal Solutions as SERS substrates}

This section presents various aspects of colloidal solutions for SERS measurements and their properties are discussed.

\subsubsection{Lee and Meisel Silver Colloids}

The workhorse of the SERS community, in terms of substrates, for the last 30 years have been citrate reduced silver colloids synthesised by what is generally referred to as the Lee and Miesel method [43]. First developed in 1982, this simple procedure yields large volumes of roughly spherical silver particles in aqueous solution. The procedure followed in this study was a modified version of the synthesis outlined in Reference [72] as follows.

$90 \mathrm{mg}$ of $\mathrm{AgNO}_{3}$, weighed out using a plastic pipete tip (to prevent reaction with metal spatulas), was added to $500 \mathrm{~mL}$ of MilliQ (18.3 M $\Omega \mathrm{cm}$ ) water in a conical flask. A clean magnetic stirrer and plastic thermometer added and the solution brought to the boil under moderate stirring and covered with aluminium foil to prevent evaporation. Upon boiling, $10 \mathrm{~mL}$ of sodium citrate (1\% weight) was added dropwise to the solution. Boiling was continued then for 1 hour until all of the $\mathrm{AgNO}_{3}$ was reduced. The resulting solution is a cloudy grey/yellow suspension which was cooled immediately in an ice bath and stored in polystyrene bottles at $4^{0} \mathrm{C}$.

Figure 3.2 shows SEM images of the nanoparticles obtained by the Lee and Meisel method (a) and (b) along with their UV-Vis extinction spectrum at one tenth concentration. The extinction spectrum exhibits a clear peak at $\sim 430 \mathrm{~nm}$ corresponding to the main dipolar resonance of a $60 \mathrm{~nm}$ diameter silver sphere, indicating that the colloids are primarily made up of spheres of this size. SEM images and the presence of the long wavelength $(\sim 500$ to $\sim 700$ ) tail in the extinction spectrum however indicate that there are many other nanoparticle geometries present in the solution along with spheres. These range from elongated rods to plates and other non-spherical shapes. The significant polydispersity of the colloids are suspected to be one reason for the often large fluctuations observed in SERS measurements; the diffusion of 

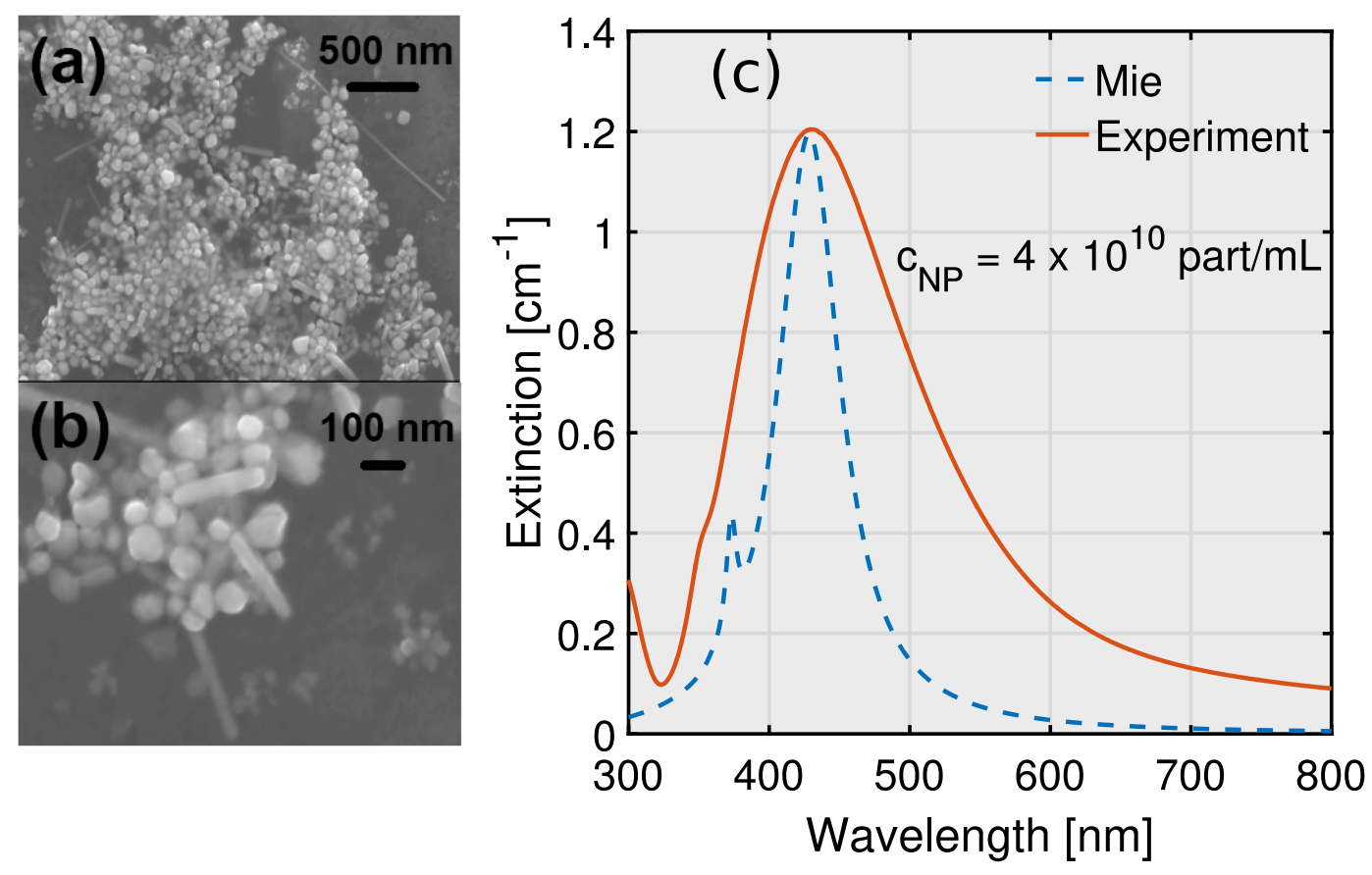

Figure 3.2: (a) - (b) SEM images of $60 \mathrm{~nm}$ silver colloids produced by the Lee and Meisel method. (c) The measured extinction spectra at one tenth concentration, along with the predicted Mie-theory extinction spectrum for a $60 \mathrm{~nm}$ silver sphere are shown on the right. There is a clear broadening of the measured extinction spectrum compared with theory due to the presence of many different shapes and sizes of nanoparticles, as evident in the SEM images. SEM images were taken from Reference [16].

colloids in and out of the laser scattering volume means that particles with largely different plasmon resonances (spheres vs rods for example) will couple differently to the incident laser and thus all molecules in the sample will not feel the same signal enhancements.

Nevertheless, the shortcomings of polydispersity are more than compensated for in Lee and Meisel colloids by the enormous enhancements they can exhibit under the right conditions and as such have been adopted over the past thirty years as the primary substrate for SERS studies and feature in many of the seminal papers on single molecule SERS $[2,3]$. For a more detailed discussion of the chemical properties of Lee and Meisel colloids one can refer to Chapter 7 of Reference [16]. 


\subsubsection{Nanocomposix Colloids}

The nanoparticles used in Chapter 6 are a much improved version of the traditional Lee and Meisel colloids that are commercially available. Produced by Nanocomposix ${ }^{1}$, the NanoXact nanoparticles are $60 \mathrm{~nm}$ diameter silver spheres, in aqueous solution and capped with citrate. The concentration of particles is roughly $0.02 \mathrm{mg} \mathrm{mL}^{-1}$ which corresponds to about $2 \times 10^{10}$ particles per $\mathrm{mL}$, or $28 \mathrm{pM}$. TEM images (provided by the manufacturer) and the measured UVVis extinction spectra are shown in Figure 3.3. As can be seen, these colloids show a dramatic improvement in size and dispersity control as compared with Lee and Meisel colloids. There are almost no non-spherical particles present and as such comparisons with Mie theory are more reliable, as seen in the theoretical extinction spectrum overlaid in Figure 3.3 (a).
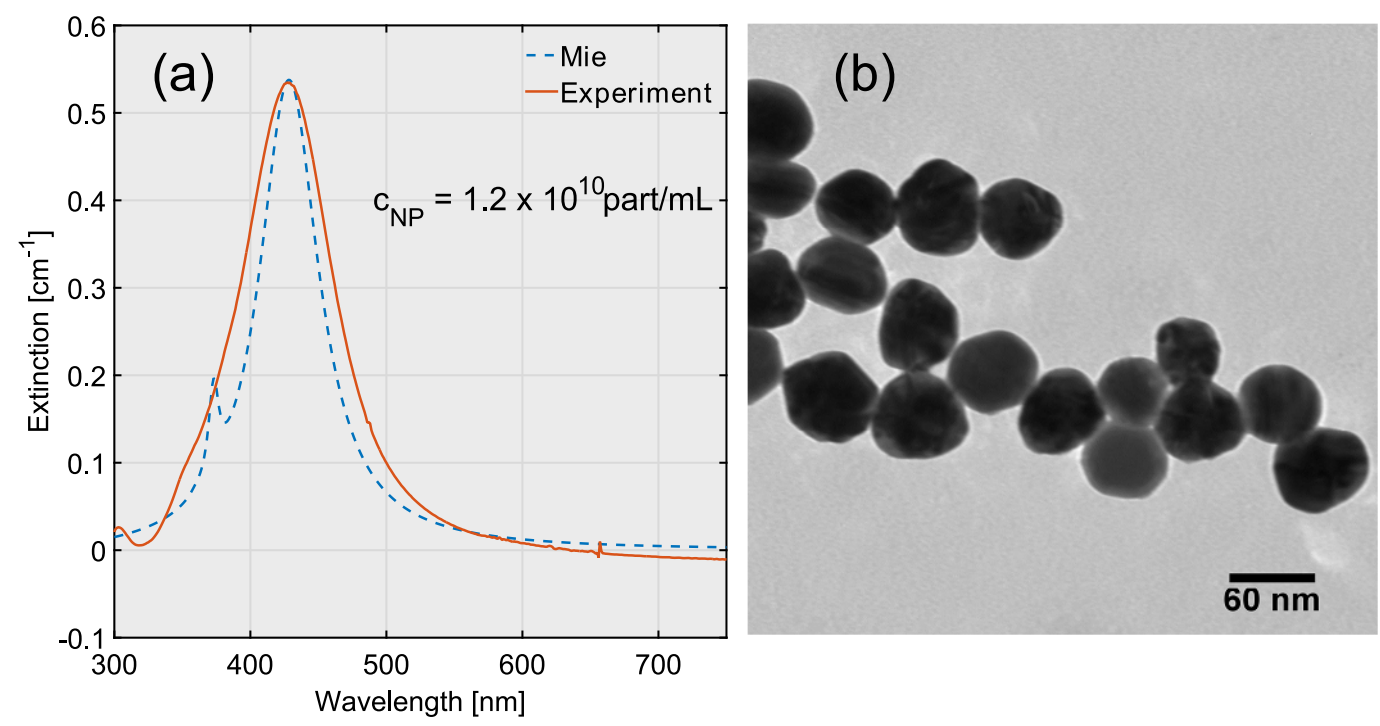

Figure 3.3: (a) The measured extinction spectra at one quarter concentration, along with the predicted Mie-theory extinction spectrum and (b) TEM images of commercially available $60 \mathrm{~nm}$ silver colloids. The measured extinction spectrum much more closely resembles the theoretical predictions due to the improved size dispersity of colloids, also seen in the TEM images. TEM images are those provided by the manufacturer.

\footnotetext{
${ }^{1}$ www.nanocomposix.com
} 
The reliability in colloid size, dispersity and concentrations provides a number of advantages; most importantly, estimations of dye coverages are significantly more accurate than with Lee and Meisel colloids, where the assumption that the solution contains only $60 \mathrm{~nm}$ spheres for concetration estimations is quite crude. The results in Chapter 6 rely crucially on the estimation of dye coverages to model the dye-dye interactions on the surface of the nanoparticles. Furthermore the differential absorbance method used in Chapter 6 relies fundamentally on the fact that dyes do not induce aggregation of the colloids. Aggregation of particles is normally evidenced as a long tail feature appearing in the 600 to $700 \mathrm{~nm}$ region of the colloid UV-Vis extinction spectrum. This feature is often very difficult to evidence with Lee and Meisel colloids since the polydispersity of the as-synthesised solution shows a broad tail in the 600 to $700 \mathrm{~nm}$ region. For the Nanocomposix particles, there is a much finer control on particle size and thus any aggregation is more easily visible in the extinction spectra.

Given these points, the move to Nanocomposix colloids as the standard substrate for SERS and dye-particle interactions removes many of the uncertanties present with Lee and Meisel colloids and allows investigations of subtle phenomena such as the results presented in Chapter 6, that require precise knowledge of the system parameters.

\subsubsection{Nanoparticle Concentration Estimations}

All colloid concentration estimations were performed by comparing the measured extinction of a solution of colloids with that predicted by Mie theory. The method is followed from [16] and is relatively straighforward: the extinction cross section, $\sigma_{\text {ext }}\left[\mathrm{cm}^{2}\right]$ of a particle can be calculated from Mie theory [68] and represents the optical strength of a single particle of radius $a$. The BeerLambert law can then be used to calculate the particle concentration, $c_{\mathrm{P}}$ by

$$
c_{\mathrm{P}}=\frac{A \ln (10)}{N \sigma_{\text {ext }} L}
$$

where $L$ is the path length of the cuvette used and $A$ is the (wavelength dependent) extinction. The concentration obtained by this method are shown 
in Figures 3.2 and 3.3 for Lee and Meisel colloids and Nanocomposix colloids respectively. Estimated particle concentrations for Lee and Meisel and Nanocomposix colloids are found to be $4 \times 10^{10}$ and $1.2 \times 10^{10}$ particles per $\mathrm{mL}$ respectively. The drawback of this method is that is does not account for the polydispersity of the sample as the Mie theory calculation assumes particles of only one size. Nevertheless given the uncertainties present in the colloid synthesis and particle shape varitions, the values obtained are a reasonable estimate in the context of SERS. Interestingly, Nanocomposix report that the NanoXact colloids have a concentration of $1.9 \times 10^{10}$ particles per $\mathrm{mL}$, indicating that the Mie-theory calculation underestimates the concentration by about $35 \%$. As such the broadening of the real colloids is not captured and a broadening scaling factor must be applied to the predicted concentrations to account for this.

\subsubsection{Achieving SERS with colloidal nanoparticles}

Figure 3.4 illustrates the steps involved in preparing a typical SERS sample with surfactant stabilised colloids. Firstly, a solution of charge stabilised nanoparticles (colloids) are mixed with an electrolyte solution. Different electrolytes can be used but here the focus is in particular on the addition of $\mathrm{KCl}$ to the solution. The electrolyte in this case plays two roles; the negatively charged chlorine ions replace the weakly bound citrate molecules [73] on the surface of the particles through chemical bonding with the silver atoms. This layer of chloride effectively cleans the nanoparticle surface of the citrate molecules. Secondly, the excess positive and negative ions in solution $\left(\mathrm{K}^{+}\right.$ and $\mathrm{Cl}^{-}$) can then reorganise themselves through electrostatic attraction and repulsion with the adsorbed chlorine layer. This diffuse layer of ions acts to reduce, or "screen" the effective charge of the first layer. The electrostatic repulsion felt between two charged colloids is then decreased, and if the screening is large enough, the attractive Van der Waals forces will cause aggregation of particles (c). By carefully controlling the concentration of electrolyte added, a metastable solution of aggregates can be reached [74] in which aggregated particles exist in solution that can be stable for long periods of time (days to weeks depending on the type of colloids). These aggregates will in principle contain a large number of "hot-spots" as described in Chapter 2, where 


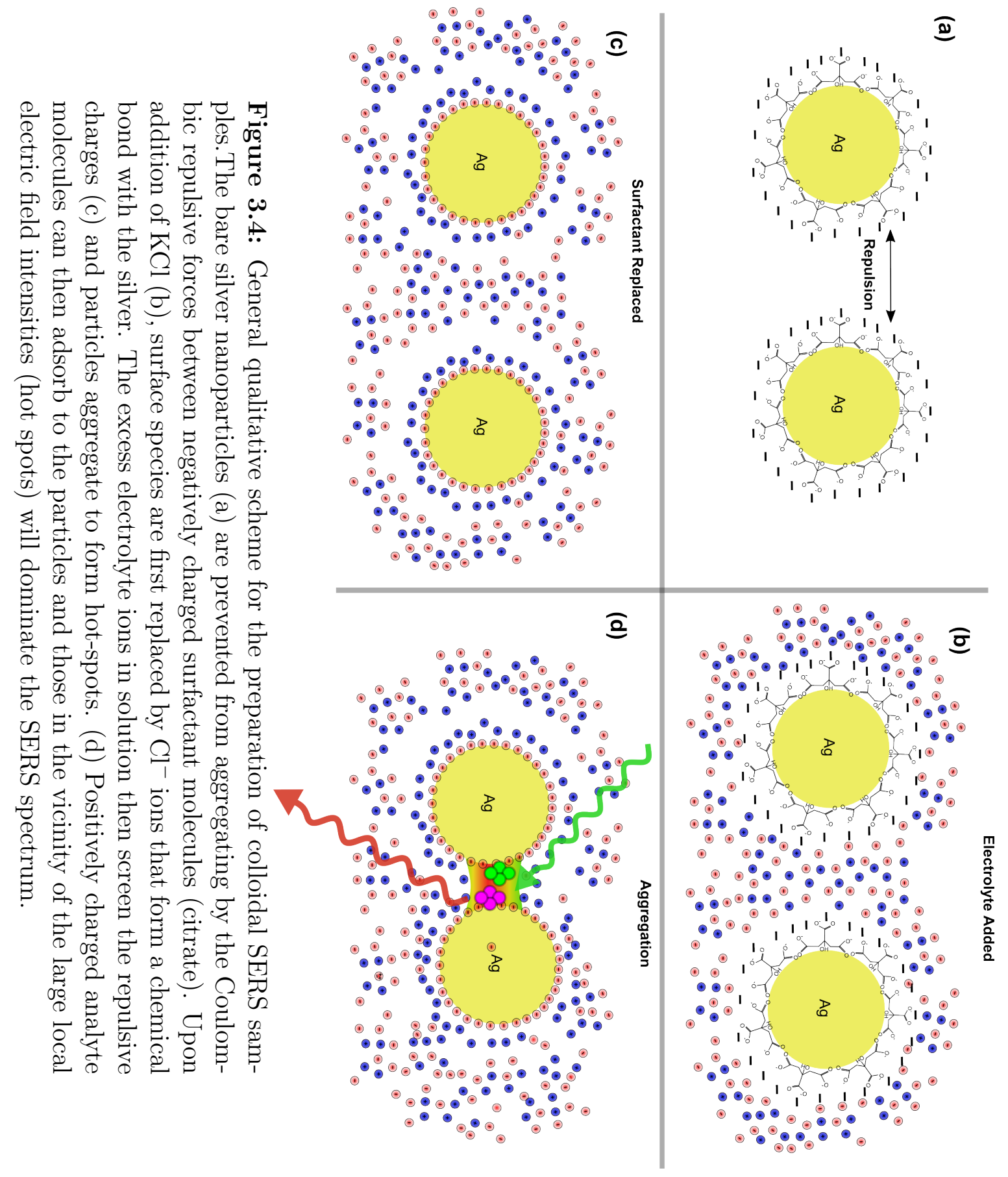


the local electric field intensities are enhanced enormously. Ideally this step only creates dimers of spheres but there can in principle exist higher-order aggregates such as trimers, quadramers and large clusters. Nevertheless, for spherical particles this step is generally necessary so as to create hot-spots and achieve large SERS EFs. Molecules added to the solution can then adsorb to the nanoparticle surface through electrostatic interactions (or possibly be chemically bound depending on the analyte). Upon excitation with a laser of the correct wavelength, these adsorbed molecules will then be subjected to the enhanced electric fields at the surface of the nanoparticles, and in turn exhibit enhanced Raman scattering as a result. The magnitude of the EFs acheivable with silver colloids are discussed later in Section 3.4.2.

The aggregation step and creation of dimers (ideally) by the addition of $\mathrm{KCl}$ to the charge stabilised colloids can sometimes, depending on the type of colloids used, be evidenced by the appearance of a long-wavelength peak in the UV-Vis spectrum of the colloid solution. Figure 3.5 illustrates a typical example of this for a solution of Nanocomposix colloids to which $\mathrm{KCl}$ is added so as to induce aggregation.

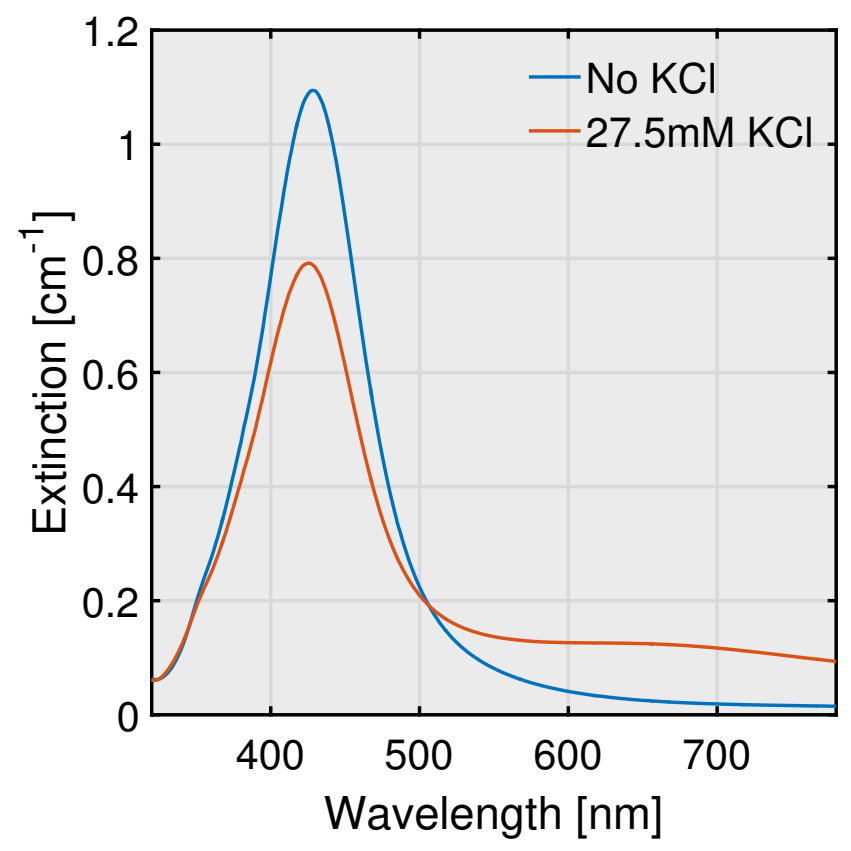

Figure 3.5: Aggregation of Nanocomposix colloids by the addition of $27.5 \mathrm{mM}$ $\mathrm{KCl}$ evidenced by a long wavelength peak in the UV-Vis exinction spectrum, indicating the presence of dimers and possibly larger clusters.

Before the addition of $\mathrm{KCl}$, the extinction spectrum displays a sharp peak 
at $430 \mathrm{~nm}$ that corresponds to the main dipolar plasmon resonance of single nanospheres in solution. Upon addition of $\mathrm{KCl}$ (here a final concentration of $27.5 \mathrm{mM}$ ), a decrease in the main peak intensity is observed along with the appearance of a broad peak between 600 and $700 \mathrm{~nm}$, in the tail of the main plasmon resonance. This peak corresponds to the exctinction spectrum of dimers (as predicted by generalised Mie-theory (Chapter 2)) and possibly higher order aggregates in solution. In general these solutions will exhibit much higher SERS EFs than the non-aggregated colloids. The prediction of SERS EFs for spheres and dimers are also discussed in Chapter 2. Important to note is that the exact details of the aggregate formation is strongly dependent on the type of colloids and electrolyte used. The example above is for $30 \mathrm{~nm}$ radius Nanocomposix colloids in water for which the aggregation regime is found to be above $25 \mathrm{mM}$ final $\mathrm{KCl}$ concentration. For Lee \& Meisel colloids (described below) the optimum conditions are found to be $10 \mathrm{mM} \mathrm{KCl}$ final concentration. The mechanisms can be understood better using DLVO theory but this is not discussed in detail here. A full explanation is given in Reference [74].

The description presented above is very rough and omits many of the finer details governing the aggregation procedure but serves to give a brief overview of the method used throughout the thesis. There are many variations on preparing colloidal samples for SERS and it would be fruitless to describe them all. Many other procedures can be found in the literature [39,75] and the reader is referred there for a more detailed review. In the remainder of the thesis, the above method, with minor variations, is the one used for sample preparation unless otherwise stated. 


\subsection{Common SERS Analytes}

Historically, SERS has been performed using aromatic dye molecules mainly due to their strong interaction with light (due to strong electronic absorption in the visible region) and their large (resonant) Raman cross sections [60]. The most commonly used dye molecules are those with $\pi$-conjugated systems of aromatic rings such as Rhodamine $6 \mathrm{G}$ and its derivatives, Nile Blue, along with other aromatic species like Crystal Violet and Malachite Green. Discussed in detail in Chapter 2, all of these molecules possess delocalized electrons across the conjugated system, due to alternating double bonds within the chromophoric group. This delocalization is responsible for absorption of light in the visible region. The structure of the dyes used in this thesis, along with their electronic absoprtion spectra are shown in the following sections. It is important also to note that all of the dye molecules used for SERS (in the specific case of negatively charged colloids that are used in this thesis) have a positively charged (cationic) end group that allows them to adsorb electrostatically to negatively charged silver nanoparticles. Negatively charged molecules tend to give very weak or no SERS signal with citrate reduced silver colloids because they do not readily adsorb to the nanoparticle surface.

The exact mechanisms that determine the optical properties of organic compounds is a field within itself and here only the most important aspects relevant to SERS are addressed. The reader is referred to the excellent overview of organic dyes in [76] for a more detailed discussion.

\section{Rhodamine 6G}

Rhodamine 6G (RH6G) is possibly the most commonly used optical dye in spectroscopy. Its many attractive properties (strong absorption cross section, solubility in water, photostability and large quantum yield) have made it ubiquitous as the standard probe for many spectroscopic applications, notably as a fluorescent standard in and in fluorescence spectroscopy for biological imaging and tracing [77]. Its original use was as a laser dye [78] due to its large quantum yield. Like most organic dyes, Rhodamine possesses a conjugated system of alternating single and double bonds in its xanthene ring [76] allowing it to 
absorb strongly in the visible region with a peak absorbance at $\sim 527 \mathrm{~nm}$ in water.<smiles>CCNc1cc2oc3cc(=[NH+]CC)c(C)cc-3c(-c3ccccc3C(=O)OCC)c2cc1C</smiles>

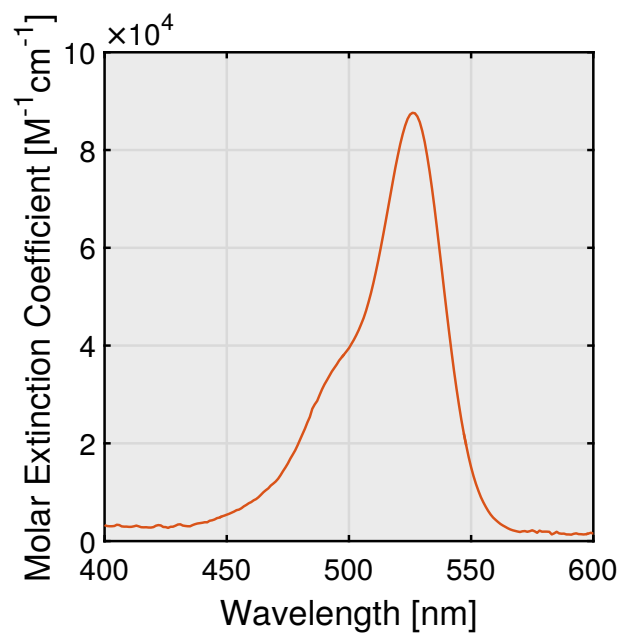

Figure 3.6: Chemical structure and absorption spectrum of Rhodamine 6G in water.

The molecular structure and UV-Vis absorption spectrum of Rhodamine 6G are shown in Figure 3.6. As a SERS probe, RH6G is problematic due to the difficulty in knowing its normal Raman cross section. Its large quantum yield in water makes performing Raman measurements in the visible region (i.e. at resonance with the molecule) extremely difficult because of the overwhelming fluorescence signal. The Raman spectrum can be detected above the fluorescence background at $633 \mathrm{~nm}$ excitation however and as such SERS enhancement factors can be calculated at this wavelength [79]. The process is much more difficult at green excitations (e.g $514 \mathrm{~nm}$ ) where ultra-fast techniques that reject the overwhelming fluorescence [60] or recently proposed polarisation difference methods [80] must be employed to measure the Raman cross sections. Nevertheless, RH6G has been extensively studied over the past 40 years in many contexts and is still the standard probe used in most SERS studies. 


\section{Nile Blue}

Nile Blue (NB), an oxazine type dye, is a derivative of a xanthene dye with one of the $\mathrm{CH}$ groups in the central ring replaced by a $\mathrm{N}$, along with an additional benzene ring that affects the shape of the absorption spectrum [76]. The molecular structure and UV-Vis absorption spectrum in water is shown in Figure 3.7. NB has its peak absorption in the red giving it its characteristic blue colour. One of the most attractive properties of NB in the context of SERS is its significantly reduced quantum yield in water [81] compared with RH6G. This feature has recently been shown to have great promise for studying resonance Raman cross sections in SERS, as the reduced fluorescence, along with novel CCD-shifting techniques allows access to the full resonance excitation profile of the Raman spectrum in water. A downside is its sensitivity to photobleaching [44] and as such laser powers must be kept low when performing SERS at resonance (e.g. $633 \mathrm{~nm}$ ) to ensure molecules are not destroyed by the incident laser. NB has also been shown to have interesting adsorption geometries on metal surfaces, which are important for determining surface selection rules in SERS [82]. This point will be relevant in Chapter 6.
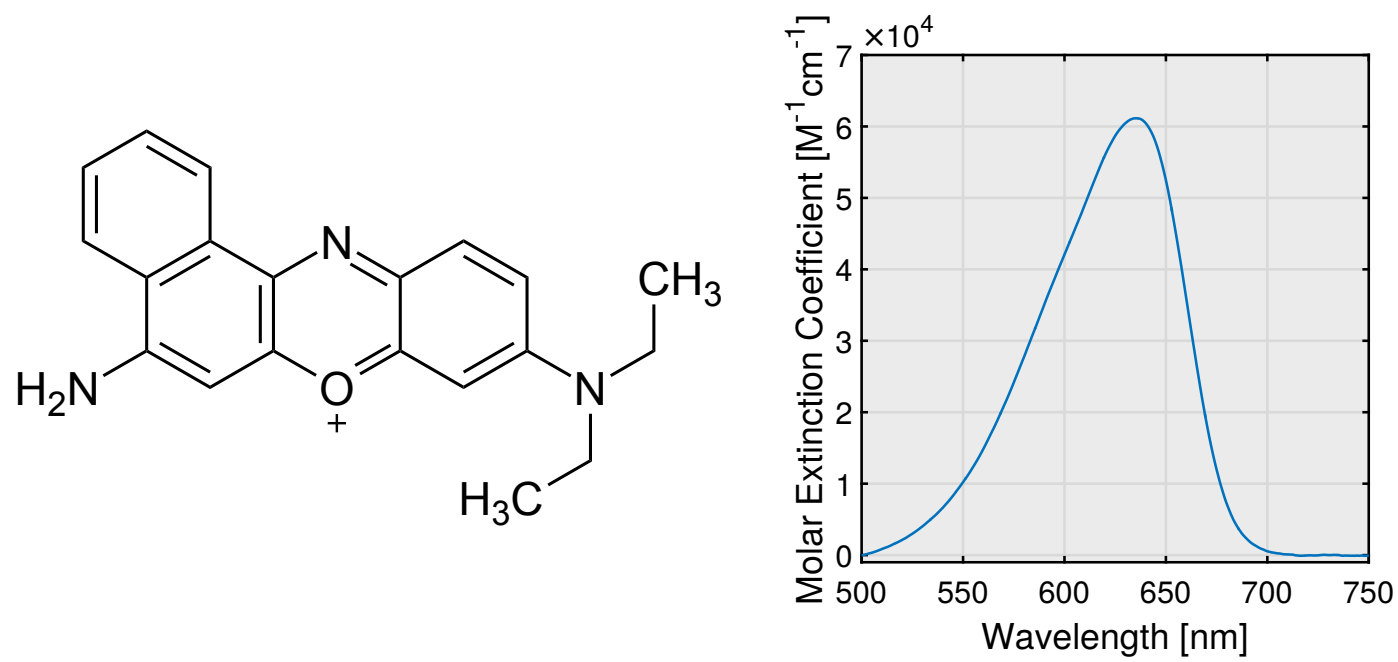

Figure 3.7: Chemical structure and absorption spectrum of Nile Blue in water. 


\section{Crystal Violet}

Crystal Violet is a member of the triphenylmethane group of organic dyes that is even more synonymous with SERS studies than Rhodamine 6G. It was used in the initial claims of single molecule SERS [2] and as such has received a wealth of attention since then. Depending on the end groups, the colour can be drastically different. Crystal Violet has a $\mathrm{D}_{3}$ symmetry and in water has a peak electronic transition at $\sim 590 \mathrm{~nm}$ with a second higher energy vibronic shoulder at $550 \mathrm{~nm}$. It is also extremely sensitive to $\mathrm{pH}$ and is commonly used as a $\mathrm{pH}$ indicator. The propeller like structure of triphenylmethane dyes is thought to be responsible for its reduced quantum yield through non-radiative relaxation by twisting conformation [83]. Like Nile Blue, this proves very useful in determining resonance Raman cross sections and relating these to SERS cross sections, as evidenced in [79].<smiles>CN(C)c1ccc(C(=C2C=CC(=[N+](C)C)C=C2)c2ccc(N(C)C)cc2)cc1</smiles>

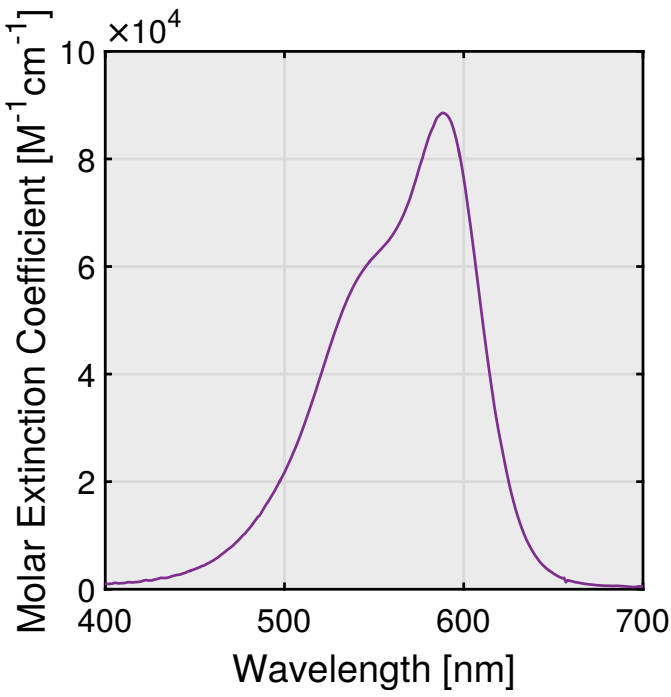

Figure 3.8: Chemical structure and absorption spectrum of Crystal Violet in water. 


\section{Rhodamine 700}

Rhodamine 700 (RH700) is similar in nature to RH6G, possessing the usual xanthene backbone, but is more closely related to Rhodamine 101 that is more planar than RH6G [78]. As the name suggests, its resonance is shifted towards the red. In fact its name comes from its peak fluorescence peak at $700 \mathrm{~nm}$. It is another laser dye [84] that is used in a similar fashion to RH6G for fluorescent tagging but fluoresces very close to the infrared, which is desirable in biological applications where cellular autofluorescence is minimised [85]. It also possesses a trifluoromethane group attached to the main xanthene backbone instead of the carboxyphenyl group of RH6G.<smiles>FC(F)(F)C1=c2cc3c4c(c2Oc2c1cc1c5c2CCCN5CCC1)CCC[N+]=4CCC3</smiles>

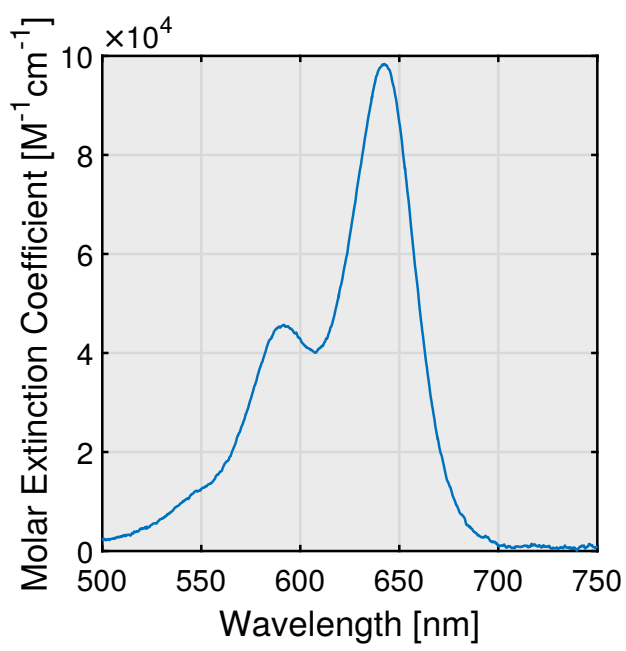

Figure 3.9: Chemical structure and absorption spectrum of Rhodamine 700 in water. 


\section{Eosin B}

Eosin B is a derivative of the well known fluorescent standard Fluorescein, where some of the hydrogens are substituted with bromines that reduce the fluorescent quantum yield of the molecule [78]. Eosin B is negetively charged so does not readily adsorb to citrate reduced silver colloids. Its main absorption peak is at $517 \mathrm{~nm}$.<smiles>O=C([O-])c1ccccc1-c1c2cc([N+](=O)[O-])c(=O)c(Br)c-2oc2c(Br)c([O-])c([N+](=O)[O-])cc12</smiles>

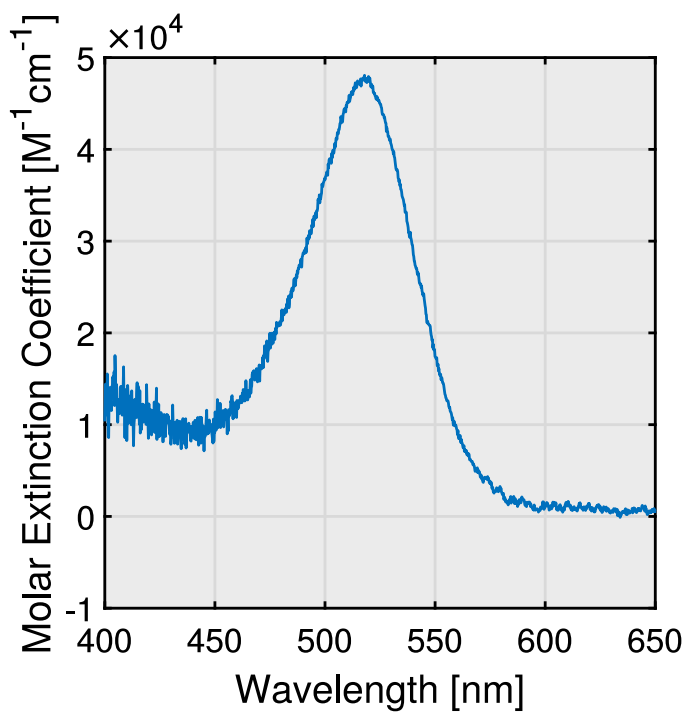

Figure 3.10: Chemical structure and absorption spectrum of Eosin B in water.

The optical properties of all dyes are summarised in Table 3.3, listing their peak absorption wavelength and molar extinction coefficient, $\epsilon$. It is noteworthy that all dyes have a similar molar extinction coefficient except for Nile Blue and Eosin having almost $60 \%$ and $50 \%$ of the values of Rhodmaine 700 respectively. 


\begin{tabular}{lcc}
\hline Dye Molecule & $\lambda_{\max }[\mathrm{nm}]$ & $\epsilon\left[\mathrm{mol} \mathrm{cm}^{-1}\right]$ \\
\hline Rhodamine 6G & 527 & $9 \times 10^{4}$ \\
Crystal Violet & 590 & $9 \times 10^{4}$ \\
Nile Blue & 635 & $6 \times 10^{4}$ \\
Rhodamine 700 & 642 & $1 \times 10^{5}$ \\
Eosin B & 517 & $5 \times 10^{4}$ \\
\hline
\end{tabular}

Table 3.1: Optical properties of dye molecules used for SERS

\subsection{Experimental Determination of SERS En- hancement Factors}

\subsubsection{Average SERS Enhancement Factors}

In this section, the practical procedure for determining SERS enhancement factors is outlined. This method was established by our group at VUW and has been adopted as the standard procedure to accurately quantify by how much the Raman signal of adsorbed analytes are enhanced by the underlying substrate. Again, the description herein is by no means complete and a full treatment of the approach can be found in Reference [12]. In particular there are a range of definitions of SERS EFs that can be used in different contexts. In the context of colloidal solutions, which are the main focus of this thesis, two enhancement factors are of interest; the average SERS enhancement, obtained from sampling over the entire colloidal solution, and the single molecule enhancement factor. These two factors are used in Chapters 4 and 6 .

The average SERS enhancements are calculated as analytical enhancement factors (AEF) as defined in Reference [12]:

$$
\mathrm{AEF}=\frac{I_{\mathrm{SERS}} / c_{\mathrm{SERS}}}{I_{\mathrm{RS}} / c_{\mathrm{RS}}} .
$$

where $I_{\text {SERS }}$ is the measured SERS intensity for a dye concentration $c_{\text {SERS }}$ and $I_{\mathrm{RS}}$ is the normal Raman intensity of a solution of possibly different (typically higher) concentration $c_{\mathrm{RS}}$ under the same experimental conditions. 
Measuring the non-SERS intensity can be problematic to perform routinely because large dye concentrations are required, and fluorescence can often swamp the underlying Raman signal. To remove the need to measure the bare Raman signal every time a SERS measurement is made, the SERS intensity can first be compared to that of a known Raman standard, normally 2-bromo-2-methylpropane (2B2MP), under the same experimental conditions (i.e. normalised to integration time, incident laser power). An effective SERS cross-section can then be inferred as:

$$
\frac{d \sigma_{\mathrm{SERS}}^{\mathrm{Ave}}}{d \Omega}=\frac{I_{\mathrm{SERS}} / c_{\mathrm{SERS}}}{I_{2 \mathrm{~B} 2 \mathrm{MP}} / c_{2 \mathrm{~B} 2 \mathrm{MP}}} \frac{d \sigma_{2 \mathrm{~B} 2 \mathrm{MP}}}{d \Omega} .
$$

where the $c_{2 \mathrm{~B} 2 \mathrm{MP}}=8.8 \mathrm{M}$ is concentration of pure $2 \mathrm{~B} 2 \mathrm{MP}$ and $d \sigma_{2 \mathrm{~B} 2 \mathrm{MP}} / d \Omega=$ $5.4 \times 10^{-30} \mathrm{~cm}^{2} / \mathrm{sr}$ is the reference Raman cross-section of the $516 \mathrm{~cm}^{-1}$ mode of $2 \mathrm{~B} 2 \mathrm{MP}$ at $633 \mathrm{~nm}[12]$.

The AEF is then obtained by comparing the average SERS cross section to that of the normal (known) Raman cross section of the molecule. This procedure requires the normal Raman cross-section, $d \sigma_{\mathrm{RS}} / d \Omega$, to only be measured once, where extreme care is taken to ensure errors are minimised [12]. For the sake of argument, the $612 \mathrm{~cm}^{-1}$ peak of Rhodamine $6 \mathrm{G}$ excited at $633 \mathrm{~nm} \mathrm{[12]}$ is used here, and has been measured to be $d \sigma_{\mathrm{RS}} / d \Omega=6.7 \times 10^{-28} \mathrm{~cm}^{2} / \mathrm{sr}$. Therefore, the AEF is obtained from:

$$
\mathrm{AEF}=\frac{d \sigma_{\mathrm{SERS}}^{\mathrm{Ave}} / d \Omega}{d \sigma_{\mathrm{RS}} / d \Omega}
$$

The procedure outlined is illustrated in Figure 3.11 where a typical SERS (a) of $25 \mathrm{nM}$ of RH6G in aggregated Lee and Meisel colloids $(20 \mathrm{mM} \mathrm{KCl})$ is measured with a x20 objective and excitation wavelength of $633 \mathrm{~nm}$. The reference spectrum of (b) is then acquired under the same experimental conditions. The integrated intensities of the SERS and reference spectra are calculated by fitting pseudo-Voigt (PS-Voigt) lineshapes (c, d) with linear backgrounds to the spectra in the region of interest and integrating the fitted lineshape. PS-Voigt profiles are common functions for fitting peak lineshapes and have been used extensively in fitting Raman peak lineshapes under various conditions [17]. The PS-Voigt profile in the presence of a linear background is given by: 

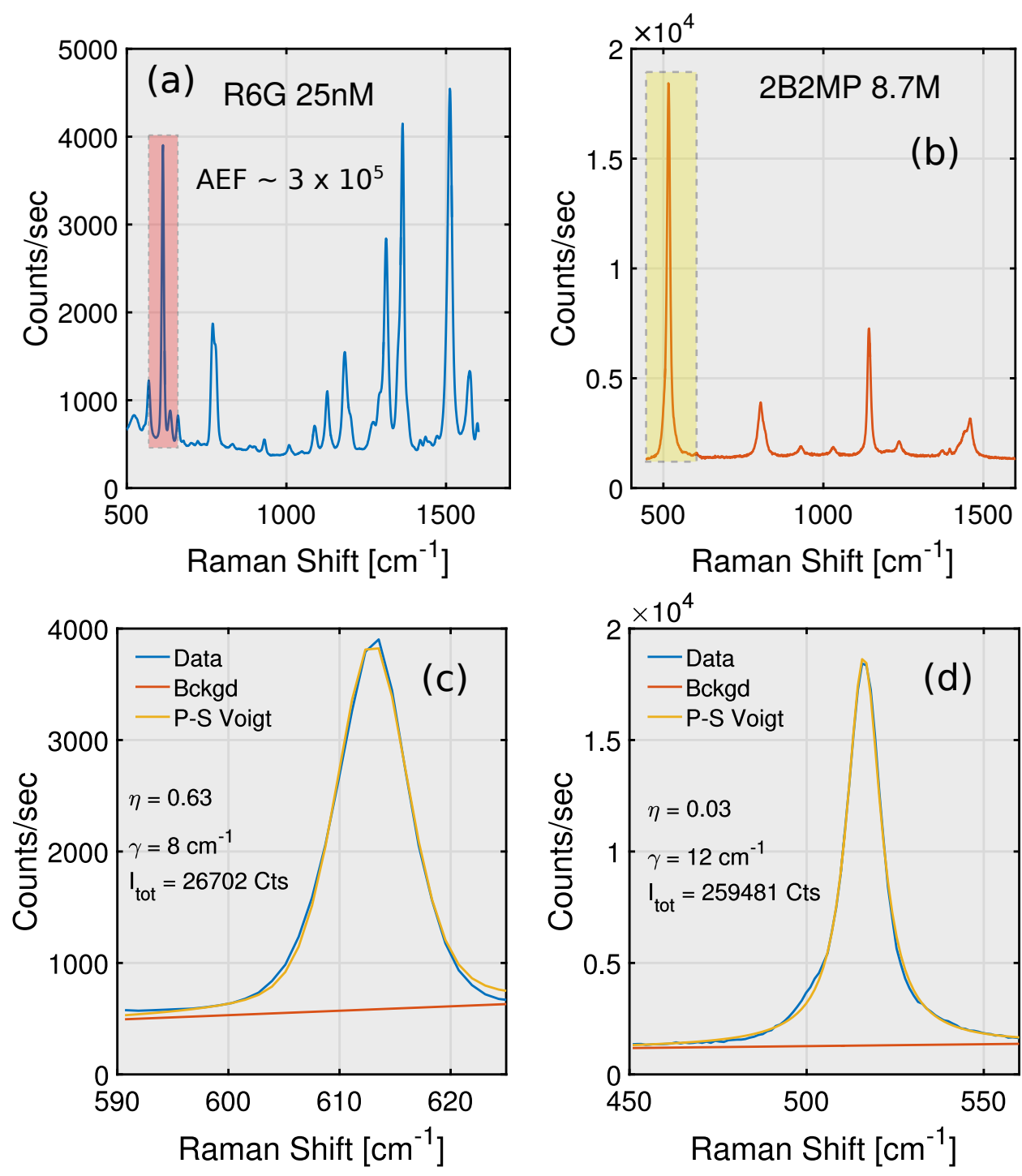

Figure 3.11: Procedure for calculating analytical enhancement factors (AEF), here for $25 \mathrm{nM}$ of RH6G in aggregated Lee and Meisel colloids (20 mM $\mathrm{KCl}$ ) with a x20 objective and excitation wavelength of $633 \mathrm{~nm}$. The top two panels are the raw (average) Raman spectra acquired for RH6G at $25 \mathrm{nM}$ and $2 \mathrm{~B} 2 \mathrm{MP}$ at $8.7 \mathrm{M}$. The shaded regions indicate the peaks used for calculations of enhancement factors. (c-d) Pseudo-Voigt profile fits to the $612 \mathrm{~cm}^{-1}$ peak of RH6G and the $516 \mathrm{~cm}^{-1}$ peak of 2B2MP using Equation 3.4.1. 


$$
V_{p s}(\bar{\nu})=(1-\eta) L(\bar{\nu})+\eta G(\bar{\nu})+m \bar{\nu}+b,
$$

where $L(\bar{\nu})$ and $G(\bar{\nu})$ are Lorentzian and Gaussian profiles centered at $\bar{\nu}_{0}$, with maximum intensities $I_{\mathrm{Max}}$ and FWHM $\gamma$ explicitly:

$$
L(\bar{\nu})=I_{\mathrm{Max}}\left[\frac{\gamma^{2} / 4}{\left.\left(\bar{\nu}-\bar{\nu}_{0}\right)^{2}+\gamma^{2} / 4\right)}\right]
$$

and

$$
G(\bar{\nu})=I_{\operatorname{Max}} \exp ^{-\left[\left(\bar{\nu}-\bar{\nu}_{0}\right)^{2} / 2 c^{2}\right]}
$$

The parameter $c=\gamma / 2 \sqrt{2 \log (2)}$ is introduced to relate the Lorentzian and Gaussian FWHM values and $\bar{\nu}$ is in wavenumbers $\left[\mathrm{cm}^{-1}\right]$. The "amount" of Lorentzian and Gaussian broadening present in the peak shape is determined by the factor $\eta$, where by construction here $\eta=0$ gives a fully Lorentzian peak with no Gaussian broadening. Peak profiles are obtained by performing (using MATLABs fminsearch function) a non-linear least squares fit of Equation 3.4.1 to the measured spectra over a suitable range of wavenumbers, $\bar{\nu}$, varying the parameters $\gamma, \eta, \bar{\nu}_{0}, I_{\mathrm{Max}}, m$ and $b$. The integrated intensity is then calculated by integrating the area under the fitted peaks to give $I_{\text {Tot }}$ for each peak.

Figure 3.11 (c-d) shows the results of fitting PS-Voigt lineshapes with linear backgrounds to the $612 \mathrm{~cm}^{-1} \mathrm{RH} 6 \mathrm{G}$ and $516 \mathrm{~cm}^{-1} 2 \mathrm{~B} 2 \mathrm{MP}$ peaks as measured. Fitting parameters are indicated along with the total intensities (in counts) showing that the 2B2MP peak is almost perfectly Lorentzian, with $\eta=0.03$ while the RH6G peak has a significant amount of Gaussian broadening. The calculation of the AEF from the PS-Voigt fitting procedure is summarised in 
3.2.

\begin{tabular}{cccccccc}
\hline Molecule & $\begin{array}{c}\text { Raman } \\
\text { Mode } \\
{\left[\mathrm{cm}^{-1}\right]}\end{array}$ & $\begin{array}{c}\gamma \\
{\left[\mathrm{cm}^{-1}\right]}\end{array}$ & $\eta$ & $\left.\begin{array}{c}I_{\text {Tot }} \\
{[\text { counts }]}\end{array}\right]\left[\mathrm{cm}_{\text {SERS }} / d \Omega\right.$ & $\begin{array}{c}d \sigma_{\mathrm{S}} / d \Omega \\
{\left[\mathrm{cm}^{2} \mathrm{sr}^{-1}\right]}\end{array}$ & AEF \\
\hline 2B2MP & 516 & 8 & 0.03 & 26702 & - & $5.4 \times 10^{-30}$ & - \\
RH6G & 612 & 12 & 0.63 & 259480 & $2 \times 10^{-22}$ & $6.7 \times 10^{-28}$ & $3 \times 10^{5}$ \\
\hline
\end{tabular}

Table 3.2: AEF calculations for the $612 \mathrm{~cm}^{-1}$ RH6G using the $516 \mathrm{~cm}^{-1}$ mode of 2B2MP as a Raman standard.

While the AEF provides the simplest method for estimating the SERS EF, it must be mentioned that it has a number of drawbacks as explained in Reference [12]. Firstly it does not account for the adsorption efficiency of molecules to the surface as it inherently assumes that in the SERS configuration, all the molecules are adsorbed to the substrate. This introduces problems when comparing EFs for different analytes that may have different affinities to the substrate surface, and when comparing EFs across different substrates. For example when comparing the EF of a gold substrate to a silver one, the analyte used to measure the EF may bind more strongly to silver than to gold, or vice versa, due its chemical structure and thus portray a skewed estimation of the relative EFs of each substrate. Other issues such as monolayer vs multilayer coverages and polarization dependencies of the EF are also not captured by the AEF definition. The problems with this approach are more important when using dried 2D substrates and the AEF lends itself well to EF estimations of colloidal solutions.

\subsubsection{SERS Spectra in Colloidal Solutions}

The method for preparing colloidal SERS samples was described in Section 3.2.1 whereby $\mathrm{KCl}$ is added to citrate-reduced $\mathrm{Ag}$ colloids after which the 
SERS analyte is added. The average SERS enhancement factors can vary by up to 6 orders of magnitude depending on a wide range of factors including, but not limited to:

- The SERS substrate material (e.g silver vs gold)

- Hot spot size/density

- Environment (dried or in solution)

- Analyte affinity to the substrate

- Excitation wavelength used.

Given that all SERS experiments performed in this Thesis are done using citrate-reduced Ag colloids, it is worth giving some typical values of the expected AEF magnitudes that are observed under various conditions. The values given are more rough figures of merit that can be used throughout the thesis to gauge what EFs should be expected for given conditions, assuming $100 \%$ adsorption of dyes.

There are essentially three SERS "regimes" for colloidal solutions that can be obtained with the standard $\mathrm{KCl}$ addition method. These are summarised in Table 3.3 below and represent those commonly measured for Lee \& Meisel. Note the AEFs here are for Rhodamine $6 \mathrm{G}$ but similar values are found for other dyes like Crystal Violet [12]. The first regime is non-aggregated colloids, whereby a small amount (typically $1 \mathrm{mM}$ ) of $\mathrm{KCl}$ is added to the colloids to remove the citrate capping layer and allow molecules to adsorb. Values obtained here are usually in the $5 \times 10^{3}-3 \times 10^{4}$ regime. At this concentration, the $\mathrm{KCl}$ is not expected to induce aggregation of colloids. The measured EFs are observed to be larger than expected from Mie-theory for a $30 \mathrm{~nm}$ radius Ag sphere. Mie-theory predicts an average SERS EF, $\langle F\rangle$ of $\sim 1300$ at $633 \mathrm{~nm}$ while measured EFs can often be an order of magnitude larger. This discrepancy is due to both the polydispersity of the colloids (there are many rods and non-spherical particles present in Lee \& Meisel colloids as seen in Figure 3.2) giving rise to a range of plasmon resonances and the fact that Mie-theory does not account for any local roughness of the particle surface 
that would act to increase the electric-field intensity further. There may also be a small number of colloidal clusters present in solution that exhibit gapmode resonances but are not visible in the UV-Vis extinction spectrum of the colloids. This highlights the lack of sensitivity of UV-Vis to the detailed structure of the solution. Nevertheless, AEFs in the $10^{3}-10^{4}$ range are typical of non-aggregated colloids.

\begin{tabular}{lcc}
\hline Colloid Regime & Experimental Conditions & Maximum AEF \\
\hline 1. Unaggregated & $1 \mathrm{mM} \mathrm{KCl}$ & $\sim 3 \times 10^{4}$ \\
& $\mathrm{c}_{\text {Dye }}<c_{\text {Sat }}$ & \\
& $10 \mathrm{mM} \mathrm{KCl}$ & $\sim 3 \times 10^{5}$ \\
2. Aggregated & $\mathrm{c}_{\text {Dye }}<c_{\text {Sat }}$ & \\
& \\
3. Unstable & $>10 \mathrm{mM} \mathrm{KCl}$ & $\sim 1 \times 10^{6}$ \\
& or $\mathrm{c}_{\text {Dye }}>c_{\text {Sat }}$ & \\
\hline
\end{tabular}

Table 3.3: Typical AEFs obtained at various experimental conditions for RH6G adsorbed to Lee \& Meisel colloids and excited at $633 \mathrm{~nm}$.

The second regime is the partially aggregated regime, where the concentration of $\mathrm{KCl}$ is increased so as to induce the formation of a metastable solution of small aggregates [74]. The concentration required is dependent on the colloids used and can be approximated using DLVO theory but for Lee\&Meisel colloids, $10 \mathrm{mM}$ final $\mathrm{KCl}$ concentration gives solutions that exhibit large, but stable SERS signals. The EFs for RH6G in this regime are generally between 1 and $3 \times 10^{5}$, consistent with Mie-theory predictions at $633 \mathrm{~nm}$ for a dimer of $30 \mathrm{~nm} \mathrm{Ag} \mathrm{spheres} \mathrm{with} \mathrm{a} \mathrm{gap} \mathrm{of} 2 \mathrm{~nm}$. Such EFs tend to be more than sufficient for single-molecule detection, given that the Mie-theory predictions of the maximum SMEF for the same gap is $\sim 5 \times 10^{7}$. 
Finally, the third condition that can be reached is the unstable regime whereby the electrostatic repulsion between colloids is no longer sufficient to overcome attractive Van der Waals forces. This is generally a result of the electrostatic screening due to the electrolyte being too large to compensate for the Van der Waals forces (see Chapter 2). For Lee \& Meisel colloids, this regime is found to be $>10 \mathrm{mM} \mathrm{KCl}$. Large EFs of the order of $\sim 10^{6}$ can be observed but the solution will tend to be unstable and collapse after a few hours.

The AEFs observed can also be dependent on the dye concentration used because the dyes also play a role in colloid aggregation. This is explained in more detail in Chapter 4. As such, the values in Table 3.3 are given for conditions where the dye concentration, $c_{\text {Dye }}$ is below that needed to induce aggregation of colloids. The third regime can in principle be reached from any of the other regimes if the dye concentration is above this limit as evidenced in Chapter 4.

\subsection{Single Molecule SERS}

\subsubsection{The Bi-analyte Technique}

All single molecule SERS (SM-SERS) measurements performed in this thesis are done using the bi-analyte SERS (BiASERS) technique. The BiASERS technique was developed in 2006 [86] as a method to dispel the uncertainties around the determination of single-molecule detection using SERS. In contrast to the original approaches $[2,3]$ of using ultra-low concentrations of analytes such that there is on average less than one molecule per colloid, the BiASERS method employs a mixture of two, spectrally different analytes to demonstrate SM-SERS detection. The major issue with using the original approach is that with such low concentrations of molecules means that the statistics for (possible) SM-SERS events are extremely poor; this is a result of two combining factors, the extreme localisation of hot-spots with high enough enhancements for SM-detection and the sparsity of molecules per colloid. Of those very few molecules in solution, only those in the regions of high enhancements will be 
detectable as SM-SERS events. The rarity of such events imposes an inherent statistical uncertainty in the true single-molecule nature of the observed events. Further exacerbating the problem is the lack of information as to whether an observed signal is from one molecule or many molecules in the same hot-spot; interpretations based on a Poisson distribution of signal intensities was proposed to infer the single-molecule nature of events but this has been shown to be an invalid claim, attributed to the lack of sampling statistics [87].

The use of two spectrally distinct analytes in the BiASERS technique automatically mitigates many of the issues associated with the low-concentration approach, because it allows one to confidently distinguish between multimolecule and single-molecule SERS events. The approach in essence relies on the idea that when two analytes, A and B, are present in the colloidal solution at equal concentrations, if the spectrum of both $\mathrm{A}$ and $\mathrm{B}$ is detected in a SERS event then it clearly originates from two or more molecules. If only the Raman spectrum of $\mathrm{A}$ is observed then it is highly likely this originates from a single (or at most a few) molecule of analyte A. The same conclusion is made for a spectrum containing only the Raman fingerprint of molecule B. The BiASERS provides a reliable proof of SM-SERS and by its nature allows the use of much larger (up to a point) concentrations of molecules; even if there are 1000 molecules of $\mathrm{A}$ and $\mathrm{B}$ on each colloid, the argument still holds that the appearance of only one species in the SERS spectra are a strong indication it originates from a single molecule. The BiASERS validity is solidified by the understanding of the long-tail distribution of EFs in typical SERS substrates [19]; for a dimer, $80 \%$ of the total SERS signal would originate from only $0.6 \%$ of the total area of the dimer, as such molecules at the points of highest enhancements contribute the most to the observed signals. If an event looks like a single-molecule event, it is highly likely that it comes from one, or at most two, molecules in these regions rather than a few molecules in regions of lower enhancements. With the use of large analyte concentrations and fast CCD based spectrometers, thousands of spectra can be collected and their SM-statistics can be analysed in detail. 


\subsubsection{BiASERS with Isotopologues}

The BiASERS technique has become the standard tool for performing and assessing SM-SERS experiments since 2006 and has shown to be successful in a range of studies $[70,88,89]$. It has even been extended recently to using four analytes [90] which gives even more insight into the overall SM-SERS statistics. The original approach [86] used two spectrally and chemically different analytes. Since then major improvements have been achieved, namely in the use of dye molecules that are isotopologues of eachother [91]. This approach is the ideal method for performing BiASERS experiments because both analytes are almost identical in nature.

The isotopologue approach used in this thesis is depicted in Figure 3.12. The two analytes used are a modified version of Rhodamine 6G, denoted RH6M, and its isotopically edited partner, d4-RH6M, that contains four deuterium atoms in place of the hydrogens on the phenyl ring. Both dyes have been fully charaterised previously in terms of their UV-Vis absoprtion spectra and Raman vibrational modes [91].

As can be seen from Figure 3.12, the Raman spectra of the isotopic partners are almost identical except for two regions; in region 1, RH6M has a prominent peak at $612 \mathrm{~cm}^{-1}$ similar to that of RH6G, while d4-RH6M has its peak shifted to $600 \mathrm{~cm}^{-1}$. A similar observation is seen in the 1300 to $1400 \mathrm{~cm}^{-1}$ region, where the d4-RH6M shows three distinct peaks instead of the two of RH6M. These two regions can then used to identify multi-molecule or single molecule events as shown on the right where examples of single molecule events of each analyte and a multi-molecule event are identified by the presence of each peak. In the bottom panel, the average spectra of all events the entire solution shows the contribution of both analytes.

SM-SERS data is obtained by acquiring a large number of consecutive spectra with integration times adjusted to account for the Brownian motion of the colloids through the scattering volume. Each individual spectrum is then fitted in the regions of interest as a linear superposition of reference spectra of each dye along with a linear background. A scatter plot of coefficients, $\alpha$ and $\beta$, is then produced where $\alpha$ and $\beta$ represent the contribution of RH6M and $\mathrm{d} 4-\mathrm{RH} 6 \mathrm{M}$ to each spectrum; an $\alpha$ value of 1 and $\beta$ value of 0 indicates a 

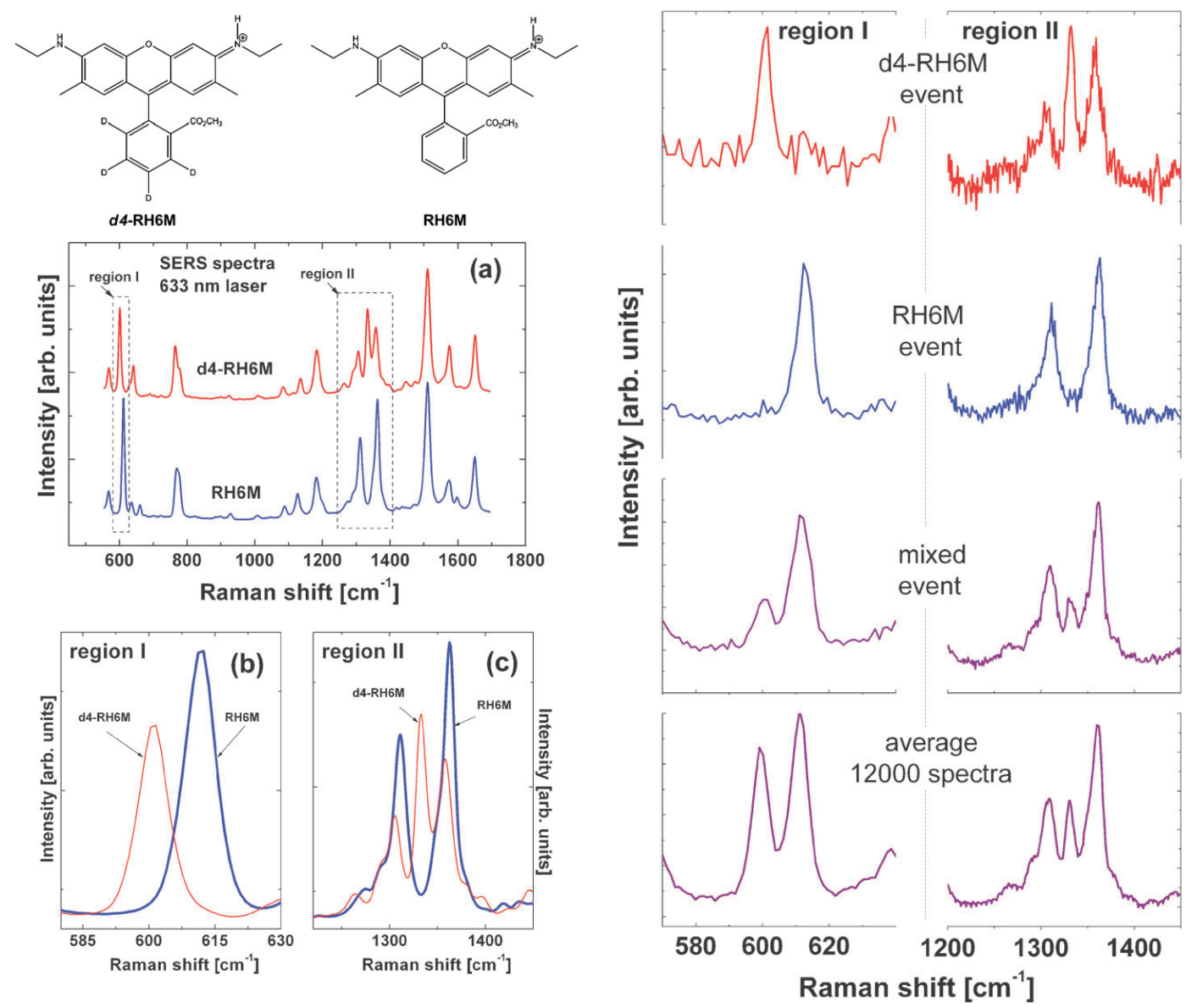

Figure 3.12: The BiASERS method with isotopically edited dye molecules. (Top) Chemical structure of the isotopic partners, RH6M and d4-RH6M. (Left) Average SERS spectra of both dyes taken at $633 \mathrm{~nm}$ excitation along with the zoomed regions of interest where the Raman spectra of both dyes are distinguishable. (c) Examples of single-molecule, multi-molecule and average SERS spectra in the regions of interest using the isotopic partners. Figure adapted from [91].

purely RH6M event and vice versa for d4-R6M. Such scatter plots are shown later in Chapter 4. 


\subsubsection{Single-molecule Enhancement Calculations}

Provided that the scattering volume of the experimental setup used has been characterised fully, the enhancement factor for single molecule SERS events can be calculated in a similar vein to that outlined for AEFs with some additional considerations.

The procedure is outlined as follows:

Using the bi-analyte technique, SM-SERS events can be identified. By defintion, the observed Raman signal then originates from one molecule in the scattering volume during the acquisition period of that spectrum. For average EF estimations, it is not required to know the actual number of measured molecules as only relative numbers appear in the calculation and they can therefore be calculated as ratio of concentrations. This is no longer the case when estimating the SERS EF corresponding to a single-molecule event. To estimate the SERS cross-section of this single molecule, it needs to be compared to the Raman intensity of a single molecule of the Raman standard 2B2MP. Measuring this is practically infeasible, but it can be inferred from a careful characterization of the effective scattering volume $V_{\text {eff }}$ as explained in Ref. [12]. In the SM-SERS experiments reported in Chapter 4, for a x100 objective at $633 \mathrm{~nm}$ the waist of the exciting beam at the focal point is $w_{0} \sim 0.63 \mu \mathrm{m}$ and the effective height of the scattering volume (as defined in [12]) is $H_{\text {eff }} \sim 21 \mu \mathrm{m}$, and therefore $V_{\text {eff }} \sim 13 \mu \mathrm{m}^{3}$. Thus the measured 2B2MP intensity, $I_{2 \mathrm{~B} 2 \mathrm{MP}}$ can be translated into the effective single molecule intensity by normalising to the concentration and scattering volume ${ }^{2}$.

As such, the single-molecule SERS cross-section for a given SM-SERS event of intensity $I_{\text {SERS }}^{\mathrm{SM}}$ is then:

$$
\frac{d \sigma_{\mathrm{SERS}}^{\mathrm{SM}}}{d \Omega}==\frac{I_{\mathrm{SERS}}^{\mathrm{SM}}}{I_{2 \mathrm{~B} 2 \mathrm{MP}} /\left(\mathcal{N} c_{2 \mathrm{~B} 2 \mathrm{MP}} V_{\mathrm{eff}}\right)} .
$$

As for the AEF, the $612 \mathrm{~cm}^{-1}$ mode of Rhodamine $6 \mathrm{G}$ and the $516 \mathrm{~cm}^{-1}$ mode of 2B2MP are used for the calculations and the SMEF is then obtained

\footnotetext{
${ }^{2}$ The conditions under which this approach are applicable, and the full details of the characterisation of the scattering volume are discussed in depth in Reference [12].
} 
as:

$$
\mathrm{SMEF}=\frac{d \sigma_{\mathrm{SERS}}^{\mathrm{SM}} / d \Omega}{d \sigma_{\mathrm{RS}} / d \Omega}
$$

Implicit in this treatment is the assumption that the events identified by the bi-analyte approach are unequivocally from single molecules. Chapter 4 highlights that the bi-analyte method can be vulnerable to erroneous identification of single-molecule events but the principles underlying the calculation of the SMEF are still valid. 


\section{Chapter 4}

\section{Dilution Effects in Colloidal Solutions}

In this Chapter, investigations are presented that were carried out on a crucial aspect of SERS in colloidal solutions that has up to now gone either unnoticed or ignored in the field. Results obtained show unequivocally that the way in which analyte molecules are diluted into colloids can have a dramatic effect on the SERS signals measured. These observations shed light on what may have been an often overlooked source of error in solution based SERS measurements in the past. The observed effects are interpreted as a competition between diffusion of molecules through the solution with electrostatic adsorption of molecules to the surface of oppositely charged nanoparticles ${ }^{1}$. By comparing average SERS signals from samples containing the same final concentration of analytes but prepared via different dilution methods, we show that when adsorption processes compete with diffusion in dilution procedures, large dilution factors should be avoided. Such dilution methods will lead to a significantly non-uniform distribution of molecules across the nanoparticle solution. This non-uniformity induces aggregation of nanoparticles that have large numbers of molecules adsorbed and thus introduces an unwanted dependence of SERS signals on analyte concentration. The implications of these

\footnotetext{
${ }^{1}$ Explicitly there are two diffusion processes occurring in the system; one associated with mixing of the dyes through the solution and one associated with the adsorption process itself. To avoid confusion, diffusion as discussed throughout this chapter is strictly limited to the mixing of dyes through the solution and does not refer to diffusion of molecules to the nanoparticle surface.
} 
findings are also discussed in relation to single molecule SERS experiments and finite element modelling is carried out to better understand the diffusion/adsorption mechanisms governing the system. The results highlight that this phenomenon has important consequences for SERS in general and also possibly in other fields where the two processes compete.

As a contextual note, these findings were also crucial to the success of the studies presented in Chapters 5 and 6 . If the effects of analyte dilution had not been investigated in detail, it is likely that the results from later studies would have suffered from irreproducibility. In this regard, the half-half dilution method proposed from this study was employed in all further works in the thesis.

Most of findings of this Chapter have been published in the Journal of the American Chemical Society [92].

\subsection{Background}

In its essence, the beauty of colloidal solutions as platforms for SERS detection is the simplicity in which a sample can be prepared. As outlined in Section 3 , the steps involved often consist of simply diluting the analyte solution into the colloids by an amount to achieve the desired final analyte concentration. For example, if $1 \mathrm{~mL}$ of a $10 \mathrm{nM}$ SERS sample of Rhodamine $6 \mathrm{G}$ was desired, one would take a stock solution of analyte (typically $10 \mu \mathrm{M}$ ) and dilute $10 \mu \mathrm{L}$ into $990 \mu \mathrm{L}$ of colloids (either aggregated with $\mathrm{KCl}$ or not, depending on the experiment). Through electrostatic adsorption, the analyte molecules will adsorb to the nanoparticles and the sample is almost immediately ready to be measured.

If one consults the massive array of articles related to SERS in colloids, it is very rare that any attention is paid to the factor by which analytes are diluted during sample preparation. Minor variations will exist depending on the analytes used; some studies will allow samples to sit overnight [93], others for a few hours prior to measurement while certain protocols have been reported to immediately shake samples after analyte addition [94] among other steps. All 
these methods no doubt improve the reliability of sample preparation and seek to alleviate the somewhat random nature of colloidal solutions but there still exists little to no mention of how analytes are diluted. Some studies will be consistent in their dilution steps [79] but the rule rather than the exception is often "A small volume of analyte stock solution was then added to nanoparticles to achieve the desired final concentration". It seems that even after many years work and the wealth of studies devoted to SERS in colloids, there has still been no established standard protocol for preparing such samples.

This work aimed to address this issue by highlighting the importance of the dilution step in colloidal sample preparation. This aspect seems to have gone unnoticed in the field up to now and the work presented vividly highlights the need to specify the method by which samples have been prepared, in particular all dilution steps. Moreover, a simple strategy is provided based on half-half dilutions for ensuring the above sources of irreproducibility are avoided. It is also suggested that this method be adopted as the standard for sample preparation of colloidal solutions for SERS.

\subsection{Experimental Conditions}

All Raman measurements were carried out at $633 \mathrm{~nm}$ exctitation in the backscattering configuration on the Jobin Yvon LabRam as described in Chapter 3. Nanoparticles used in this study were the citrate-reduced Lee and Meisel colloids as also described in Chapter 3. For all average SERS measurements, spectra were acquired with a $\times 20 \mathrm{NA}=0.5$ immersion objective (spot size $\sim 5 \mu \mathrm{m}$, scattering volume of $\sim 2000 \mu \mathrm{m}^{3}$ ) integrating over $100 \mathrm{~s}$ to ensure averaging over many colloidal particles. To ensure photobleaching of dyes was not an issue, laser power was varied as required by use of neutral density filters. For Nile Blue, which is particularly prone to photobleaching the laser power was $0.05 \mathrm{~mW}$ (ND2) and $0.5 \mathrm{~mW}$ for Rhodamine 6G. For SM-SERS measurements, spectra were acquired with a $\times 100 \mathrm{NA}=1.0$ water-immersion objective (spot size $\sim 1.2 \mu \mathrm{m}$, scattering volume of $\sim 13 \mu \mathrm{m}^{3}$ ), consecutively with each $0.1 \mathrm{~s}$ integration time. Average spectra for the non-mixed analytes, R6M and d-R6M, were also measured to facilitate the analysis. Each individual spectrum is then fitted within the spectral region of interest $\left(550\right.$ to $\left.700 \mathrm{~cm}^{-1}\right)$ as 
a superposition of these two reference spectra and a linear background using a linear least square fit. The respective intensities of R6M and d-R6M for each spectrum is simply deduced from those fits and expressed as an equivalent SM-SERS EF (meaning the SM-SERS EF assuming only one molecule contributes to the signal). These are calculated as in Ref. [12] where the bare Raman cross-sections for R6M and d-R6M were measured in [91]. Further details about the SERS EF calculations are provided Chapter 3.

\subsection{Results}

\subsubsection{The Effect of Dilution on Average Enhancement Factors}

When preparing active colloidal solutions for SERS, as described previously, it is generally assumed that, providing basic rules are followed, the exact procedure is irrelevant as long as the final concentrations (i.e. NPs, SERS probe and aggregating agents) are the same. Figures 4.1 and 4.2 both demonstrate that such an assumption is clearly wrong, at least in certain contexts.

In Figure 4.1 (a) the average SERS spectra for two samples of $100 \mathrm{nM}$ Nile Blue in Lee and Meisel colloids at $633 \mathrm{~nm}$ excitation are shown. Here colloids are premixed with $1 \mathrm{mM} \mathrm{KCl}$. At this concentration, the $\mathrm{KCl}$ does not induce aggregation but is used to remove the citrate layer on the NPs (which is replaced by chloride ions $[95,96]$ and thereby facilitate analyte adsorption. For the sake of argument only the $595 \mathrm{~cm}^{-1}$ peak is used for intensity and enhancement factor estimations.

Immediately apparent from the peak intensities of both samples is the significantly larger signal from the green curve, despite both samples containing on average the same number of Nile Blue molecules. The intensity of the former is almost 4.5 times larger than that of the latter despite all concentrations being identical. To illustrate this, the detailed preparation of each sample is reproduced below: 

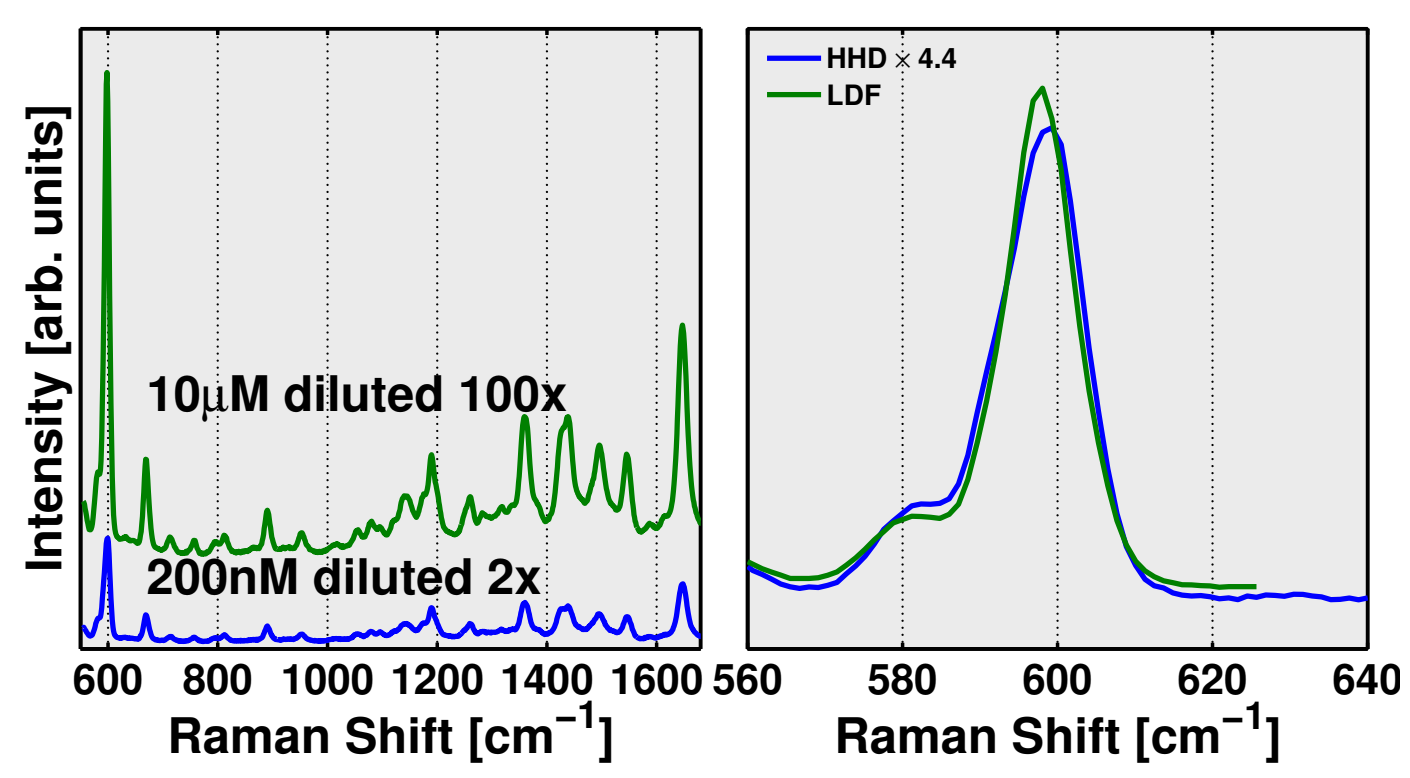

Figure 4.1: (a) Full SERS spectra of $100 \mathrm{nM}$ Nile Blue samples prepared by two different dilution methods, taken at $633 \mathrm{~nm}$ excitation. The blue curve was prepared by the "Large dilution factor" method and green by the "halfhalf" method. (b) Inset of the $595 \mathrm{~cm}^{-1}$ region showing the same spectra as in (a) but here a linear fit of the HHD method to the LDF method has been performed, resulting in a scaling factor of $\sim 4.4$. 
- Sample 1: $\left.[250 \mu \mathrm{L} \mathrm{Ag} \mathrm{colloid}+250 \mu \mathrm{L} 2 \mathrm{mM} \mathrm{KCl}]+490 \mu \mathrm{L} \mathrm{H} \mathrm{H}_{2} \mathrm{O}\right]+$ $10 \mu \mathrm{L} 10 \mu \mathrm{M}$ NB.

- Sample 2: $[250 \mu \mathrm{L} \mathrm{Ag}$ colloid $+250 \mu \mathrm{L} 2 \mathrm{mM} \mathrm{KCl}]+500 \mu \mathrm{L} 200 \mathrm{nM}$ NB.

From the details of the two preparations, it is clear that in Sample 1, the dye has been diluted by a factor of 100 to achieve the final concentration of $100 \mathrm{nM}$. This consists of adding a small volume $(10 \mu \mathrm{L})$ of $10 \mu \mathrm{M}$ stock to the colloids/ $\mathrm{KCl}$ mix. In contrast, in sample 2 the dye is sequentially diluted down to twice the final concentration and then mixed (in a 1:1 ratio) with the colloids $/ \mathrm{KCl}$ solution. In principle the only difference between samples is the way in which the dye has been diluted, yet there is a 4.5 times discrepancy between signal intensity. Figure 4.1 (b) shows the same data for Nile Blue in the $595 \mathrm{~cm}^{-1}$ region, with the spectra overlaid for ease of comparison.

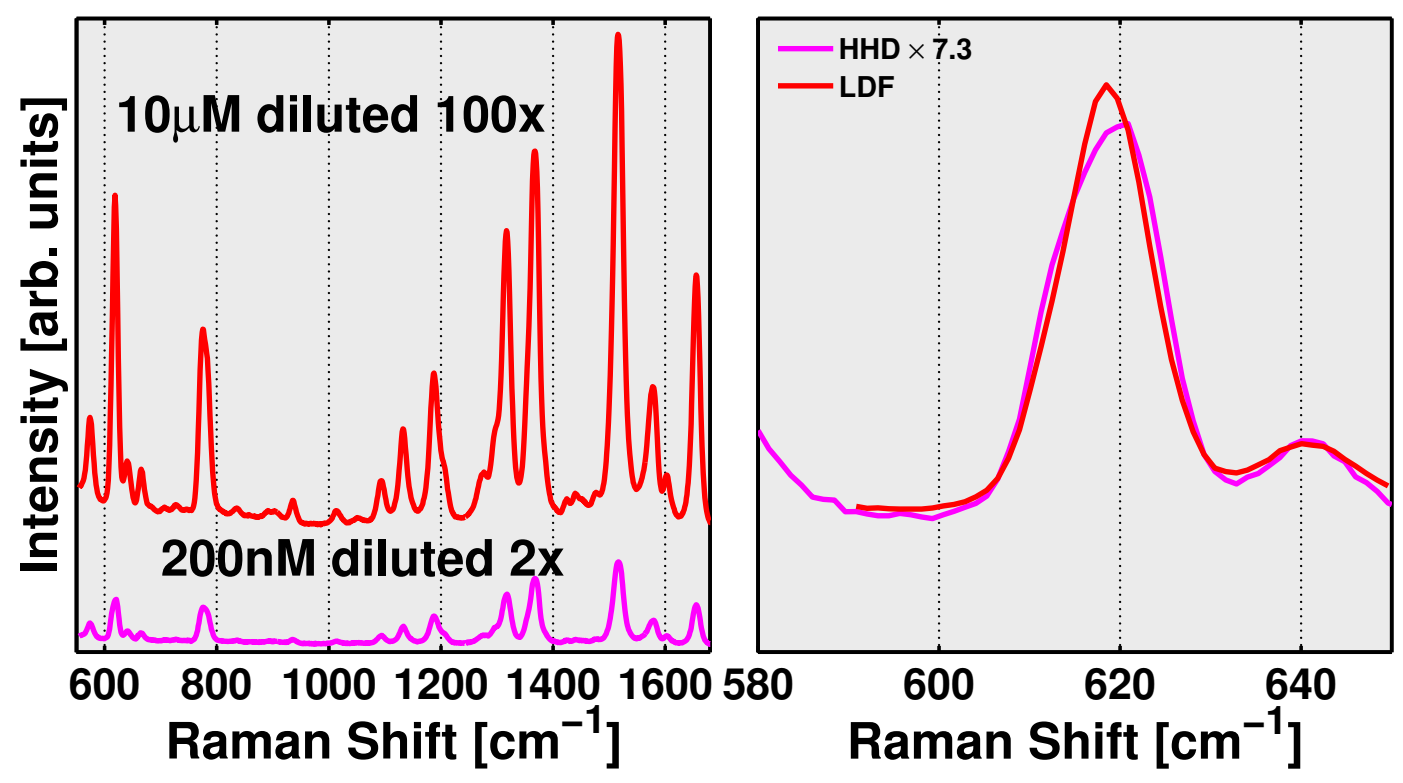

Figure 4.2: (a) Full SERS spectra of $100 \mathrm{nM}$ Rhodamine 6G samples prepared by two different dilution methods, taken at $633 \mathrm{~nm}$ excitation. The red curve was prepared by the "Large dilution factor" and pink by the "half-half" method. (b) Inset of the $612 \mathrm{~cm}^{-1}$ region showing the same spectra as in (a) but here a linear fit of the HHD method to the LDF method has been performed, resulting in a scaling factor of $\sim 7.3$.

Even more dramatic results are seen for Rhodamine 6G prepared in the same way as the Nile Blue samples. The spectra in Figure 4.2 show a factor 
of $\sim 7$ difference in signal intensity between the two preparation procedures. Note that for Rhodamine $6 \mathrm{G}$ we use the $612 \mathrm{~cm}^{-1}$ peak for comparison. Figure 4.2 (b) shows the same data the $612 \mathrm{~cm}^{-1}$ region.
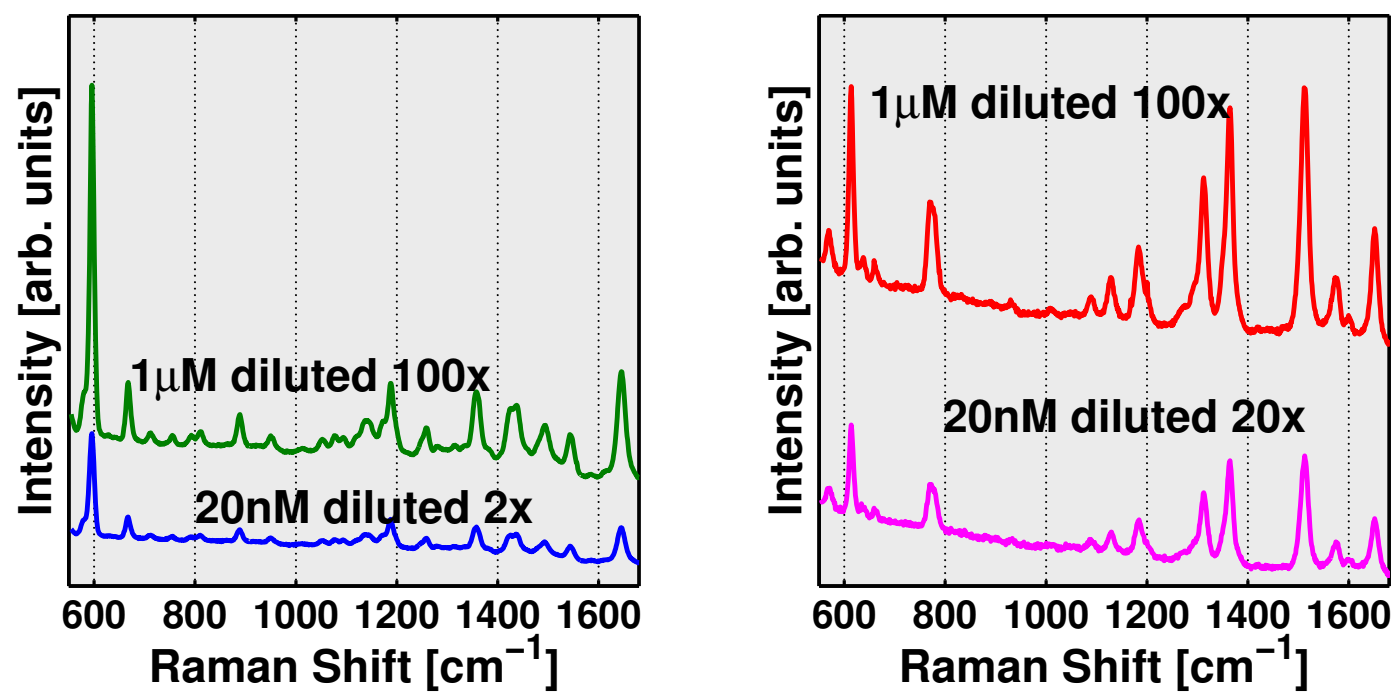

Figure 4.3: (a) SERS spectra of $10 \mathrm{nM}$ Nile Blue samples prepared by two different dilution methods, taken at $633 \mathrm{~nm}$ excitation. The blue curve was prepared by the "Large dilution factor" method and green by the "half-half" method. (b) The same data for Rhodamine 6G

Interestingly, the effect of sample dilution is less pronounced when the starting concentration of analyte is decreased as shown in Figure 4.3, where all starting (and therefore final) concentrations of dyes were decreased by a factor of 10. Here the increase in intensity between equivalent $100 \times$ and $2 \times$ dilution samples is only a factor of about $\sim 3$ for NB and $\sim 2$ for R6G. 


\subsubsection{Diffusion vs Adsorption}

The proposed explanation for these observations is based on the intuitive assumption that the kinetics for dye adsorption on the NPs are much faster than typical molecular diffusion times in the solution. Many SERS dyes (including R6G and NB) are cationic dyes to ensure strong adsorption onto the negatively charged metallic colloids through electrostatic interaction. Such a strong interaction is expected to result in very fast (sub-second) adsorption, at least in the early stages (low dye coverage) where steric hindrance does not play a role.

In contrast, dye diffusion through a typical solution of $1 \mathrm{~mL}$ occurs on time scales of minutes or more. Indeed, typical diffusion coefficients for common dyes in water are of the order of $D=10^{-5} \mathrm{~cm}^{2} / \mathrm{s}$, meausured by both Fluorescence Correlation Spectroscopy and Pulsed Field Gradient NMR [97]. These values correspond to a diffusion time through a distance $L=1 \mathrm{~mm}$ of the order of $\tau=L^{2} / D=1000 \mathrm{~s}$. The values quoted from the literature for diffusion coefficients of dyes are measured in water, and assume that no dimerization of dyes is present. This is not a concern in the conditions used here, as Rhodamine $6 \mathrm{G}$ is known to dimerise only above concentrations of $100 \mu \mathrm{M}$ [97]. In SERS measurements, dye concentrations rarely exceed $1 \mu \mathrm{M}$ and dimers are not expected to be present in solution. The colloids used in SERS are also diluted in solutions of $\mathrm{KCl}$ ( 1 to $20 \mathrm{mM}$ regime) which may affect the diffusion times but Reference [97] also found that varying the ionic concentration of the

solution from $10^{-4}$ to $10^{-2} \mathrm{M}$ had no significant effect on $D$ so it is reasonable to assume that the dye has the same $D$ as it does in water.

The slow diffusion coefficient of dyes is easily observed by injecting a small volume of high-concentration dye into a container of water and observing by eye the colored dye very slowly diffusing throughout the entire volume. In fact, diffusion-driven mixing is so slow that in most situations, convection effects will dominate and speed up the process; these include the unavoidable mechanical disturbance of injecting one solution in the other, and also possibly deliberate shaking/mixing. Even then, shaking or stirring can be expected to decrease the mixing time to a few seconds at best and should remain much slower than adsorption. If that is the case, then the majority of analytes are 
adsorbed before any diffusion/mixing occurs, i.e. in effect, they are adsorbed before being diluted to their final concentration. This then results in very different outcomes, depending on the starting concentration (or equivalently on the dilution factor).

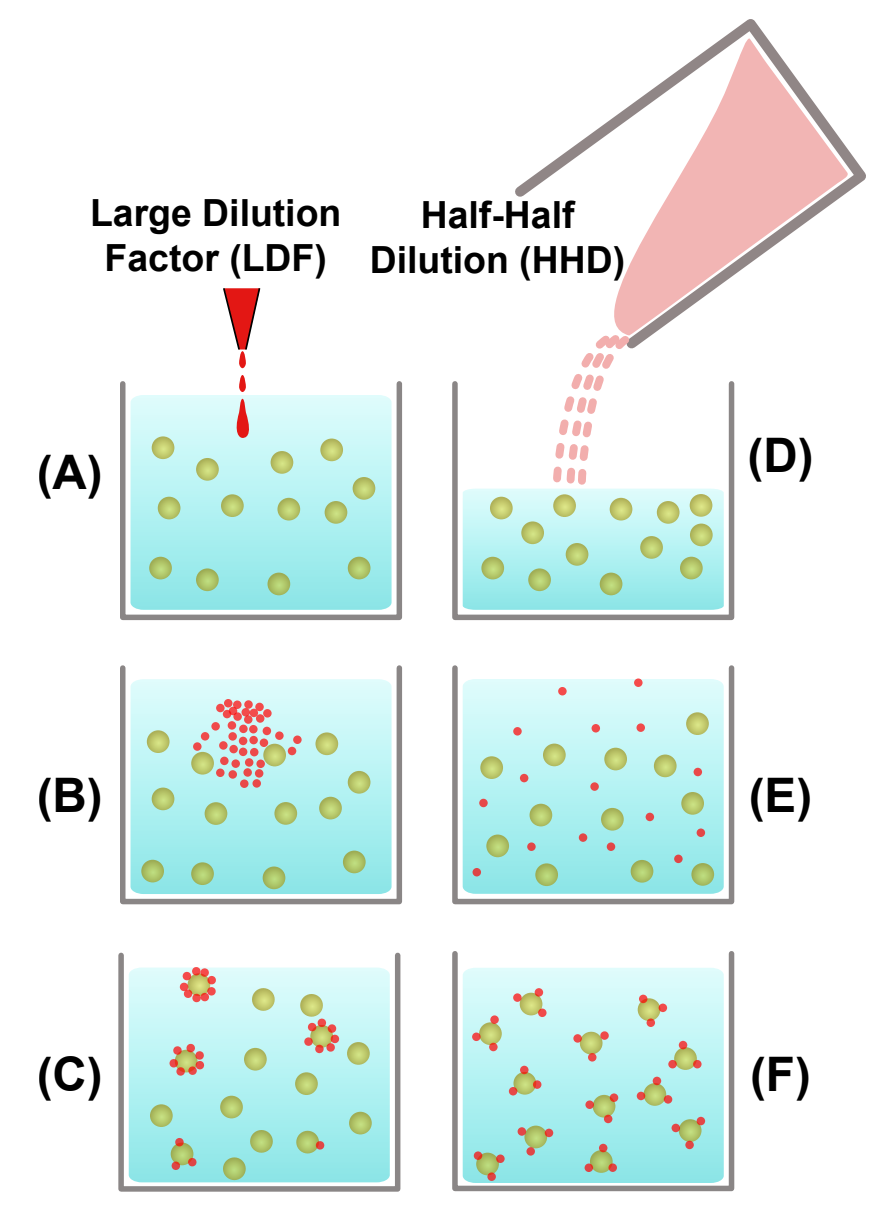

Figure 4.4: Schematic showing how two different dilution methods may lead to drastically different distribution of molecular coverage (number of analytes per colloid). The large dilution factor (LDF) method shown on the left (A-C) is typical of the majority of reported SERS experiments, while the half-half dilution (HHD) method shown on the right (D-F) is the method recommended in this work to obtain uniform molecular coverage.

Figure 4.4 schematically illustrates this effect. (A-C) depict the adsorption/mixing process in the case of large dilution factors (LDF), which seems to be the most commonly used method for preparing colloidal SERS samples. The final dye concentration is achieved by (A) diluting a small volume of dye (for example $10 \mu \mathrm{L}$ of $10 \mu \mathrm{M}$ ) in a large volume of colloids (for example $990 \mu \mathrm{L}$ corresponding to a colloid to dye volume ratio of 99:1). Before mixing, the 
dye molecules are at a much higher local concentration $(10 \mu \mathrm{M})$ in the small region they were injected into the sample (B). Because adsorption occurs before diffusion/mixing, this results in a highly non-uniform distribution of the analytes on the NPs $(\mathrm{C})$, where only a small proportion of the NPs capture a large number of molecules (potentially 1\% of the NPs have a molecular coverage 100 times larger than the expected average). This effect can be mitigated to a large extent by employing small dilution factors, ideally half-half dilutions (HHD), as shown in Fig. 4.4(D-F). The same final dye concentration $(100 \mathrm{nM})$ is achieved by mixing equal volumes of dye $(500 \mu \mathrm{L}$ of $200 \mathrm{nM})$ and colloids $(500 \mu \mathrm{L})$ as shown in (D). Convection-driven diffusion during mixing of the two volumes ensures in a first approximation that the dyes are dispersed throughout the sample uniformly (E) and each dye molecule then adsorbs to the surface of the colloids through electrostatic interaction producing uniform molecular coverage throughout the sample (F).

\subsubsection{Dynamics of Molecular Adsorption on Nanopar- ticles}

To support the diffusion vs adsoprtion hypothesis, it is demonstrated experimentally that molecular adsorption in the present system does occur on timescales faster than typical mixing times of a small volume into a larger one (which are of the order of seconds or larger). For this, the fact that the fluorescence signal of NB (or any fluorescent molecule) is quenched upon adsorption onto the metallic NPs is exploited. Fluorescence quenching is a well known phenomenon in SERS $[44,98]$ and is mainly understood as occurring due to non-radiative relaxation into the metal, the lifetime of which is much shorter (ten of femtoseconds) than typical fluorescence lifetimes of organic dyes (tens of nanoseconds) [98]. As such, any decrease in the fluorescence intensity in colloids when compared with a water solution of same concentration then clearly indicates that adsorption has started to occur.

An experiment was designed that consisted of first pre-mixing colloids with $1 \mathrm{mM} \mathrm{KCl}$ as standard, and adding a solution of $20 \mathrm{nM}$ Nile Blue (in the halfhalf method) to the colloids in situ under the microscope objective. Spectra were acquired while the mixing was performed so as the fluorescence signal of 


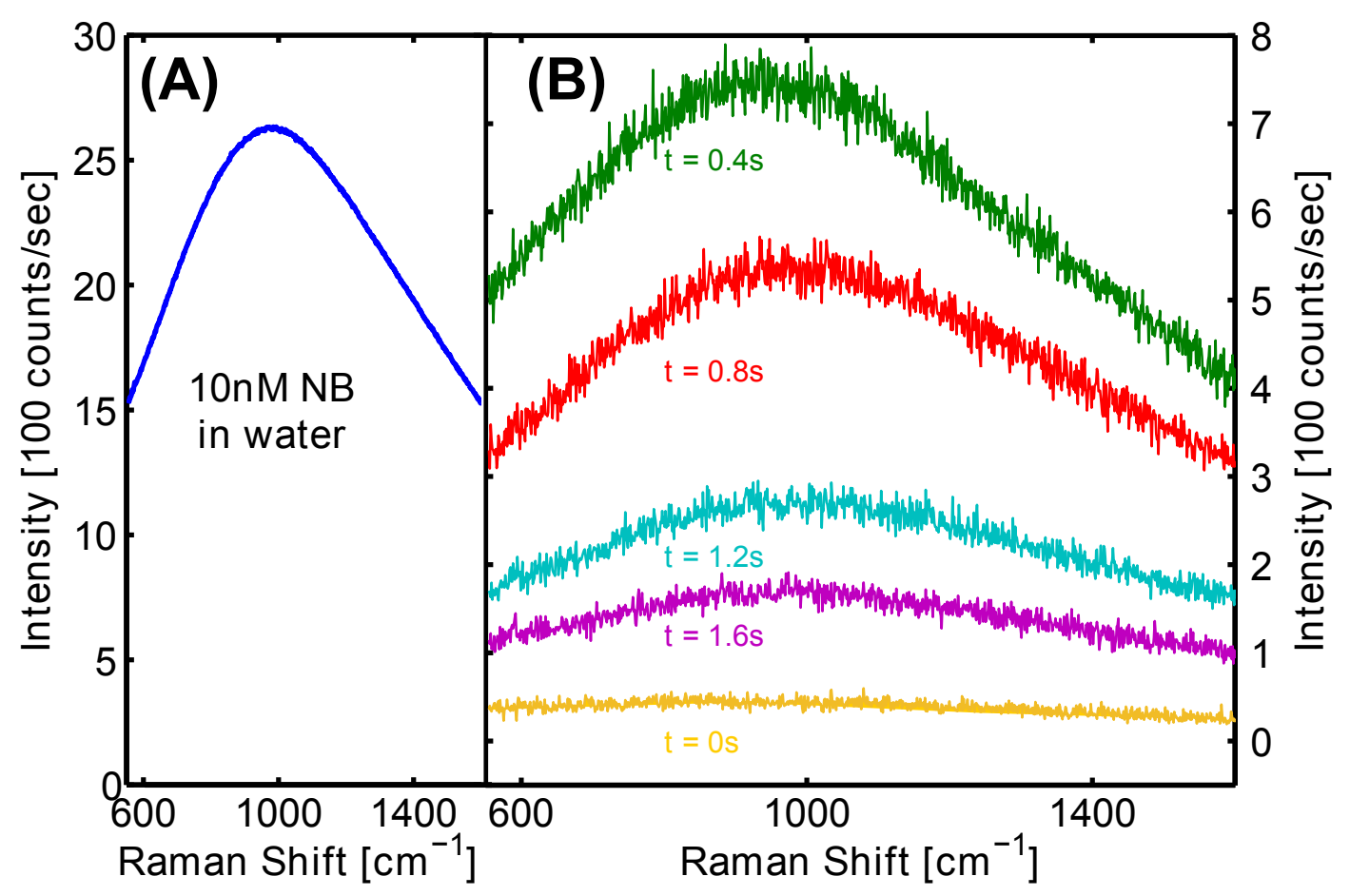

Figure 4.5: Dynamics of adsorption. (A) Reference fluorescence spectrum for $10 \mathrm{nM}$ NB in water. (B) Fluorescence/Raman spectra of $\mathrm{Ag}$ colloids in $10 \mathrm{mM}$ $\mathrm{KCl}$ to which (during measurement) an equal volume of $20 \mathrm{nM} \mathrm{NB}$ (to obtain $10 \mathrm{nM}$ NB concentration) is added. The five spectra obtained continuously (with a step of $0.4 \mathrm{~s}$ ) at the time of mixing are shown: one just before $(\mathrm{t}=$ $0 \mathrm{~s}$ ), and the first four just after. 
the added molecules could be monitored over time. The fluorescence signals were then compared to that of the same concentration in water (i.e. no colloids present). As shown in Fig. 4.5, strong fluorescence quenching is indeed observed in the first spectrum measured (i.e. within less than a second) following mixing of the dye and colloid solution. In (A), the fluorescence spectrum of $10 \mathrm{nM}$ NB in water excited at $633 \mathrm{~nm}$ shows a peak intensity of about 2500 counts per second. In (B), it is evident that even in the first spectrum right after addition of $\mathrm{NB}(\mathrm{t}=0.4 \mathrm{~s})$, the fluorescence is at most $1 / 3$ of what it should be in water and reduces further to $1 / 10$ after $1.2 \mathrm{~s}$, indicating that the majority of NB molecules are adsorbed on a sub-second time scale. The adsorption process is therefore faster than can be resolved in such experiments (i.e. subsecond). Also worthy to note is the appearance of the $595 \mathrm{~cm}^{-1}$ Raman peak of NB after $1.6 \mathrm{~s}$. Because of the strong dependence of SERS on proximity to the metal, the appearance of the Raman spectrum of NB indicates the molecules are adsorbed close enough to the surface where the local electric field is large enough to enhance the Raman signal. While the quenching of fluorescence could potentially be due to reabsorption in the dense colloidal solution, the optical absorption by the colloid solution in this wavelength range where Nile Blue fluoresces (650 to $700 \mathrm{~nm}$ ) is at most $10 \%$ and cannot explain the observed intensity drops.

\subsubsection{Effects of Analyte Concentration on Aggregation of Nanoparticles}

In itself, the strong non-uniformity in molecular coverage as predicted later in Section 4.3.5 and illustrated in Figure 4.4(F) should not result in any difference in the average SERS signal, unless the SERS EF depends non-linearly on the molecular coverage. In colloidal SERS solution, there is an obvious mechanism providing such a non-linear dependence: dye-induced colloid aggregation [99]. It is well established that aggregated colloidal solutions exhibit much larger SERS EF [31, 95, 96, 100-102] (up to a point where over-aggregation results in complete collapse/sedimentation of the colloidal solution). Aggregation is typically induced by the addition of electrolyte, which screens the electrostatic repulsion between the negatively-charged colloids. Aggregation can also be induced by adsorption of positively-charged analytes [95,96,103] (like NB and 

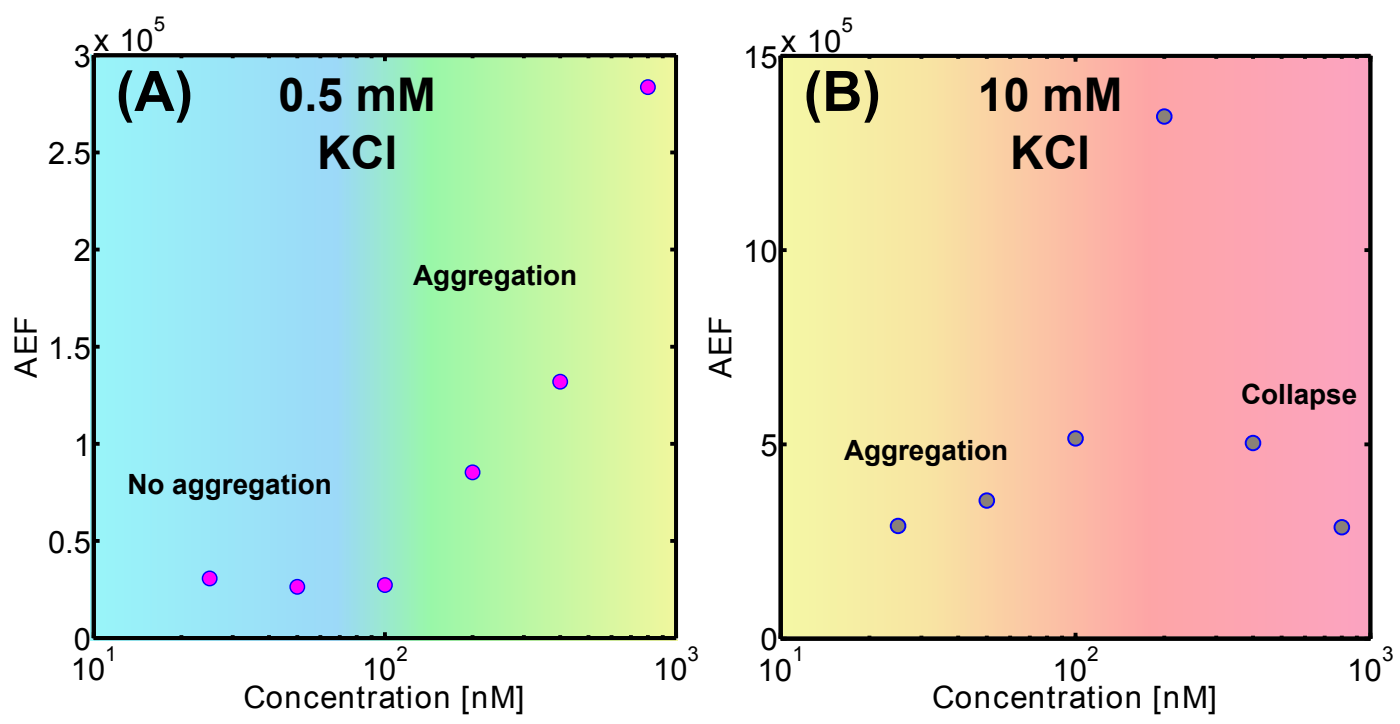

Figure 4.6: Measured average SERS EF at $633 \mathrm{~nm}$ excitation as a function of dye concentration for R6G in Ag colloid solution either (A) non-aggregated (in $0.5 \mathrm{mM} \mathrm{KCl}$ ) or (B) pre-aggregated in $10 \mathrm{mM} \mathrm{KCl}$.

R6G) as this will reduce the net negative charge of the NPs. As the molecular coverage increases, the net charge is further reduced and the aggregation is further favored. One therefore expects an increase in average SERS EF as a function of molecular coverage as a result of analyte-induced aggregation.

This phenomenon is evidenced experimentally in Fig. 4.6. The average SERS EF of R6G adsorbed to silver nanoparticles is measured as a function of dye concentration in two regimes; in (A) dyes are added to colloids in $0.5 \mathrm{mM} \mathrm{KCl}$ (so as to replace the surface citrate layer but not cause aggregation of colloids) and in (B) dyes are added to colloids in $10 \mathrm{mM} \mathrm{KCl}$ (so as to pre-aggregate the colloids, as most SERS studies are performed). Previous studies [74] have shown this to be the optimal concentration of $\mathrm{KCl}$ for producing large SERS EFs with Lee \& Meisel colloids. All samples were prepared by using the half-half dilution method to ensure uniform surface coverage of colloids, whose final concentration is $1 / 4$ of the as-synthesized solution. For unaggregated colloids (A), the EF for dye concentrations of 25, 50 and $100 \mathrm{nM}$ are essentially equal, and have magnitudes expected of non-aggregated $60 \mathrm{~nm}$ Ag colloids $\left(\sim 3 \times 10^{4}\right)$; in other words, the SERS intensity scales linearly with concentration. However, above $100 \mathrm{nM}$ (which we here estimate to correspond to about 6000 dyes per particle), the EF increases significantly from 
about $3 \times 10^{4}$ up to $3 \times 10^{5}$, an increase that can be attributed to dye-induced colloid aggregation. For pre-aggregated colloids (B), the effect of KCl-induced aggregation is clearly evident in the order of magnitude difference (10 times larger) in SERS EF at the lowest concentration (25 nM). Moreover, because the colloids are already partially aggregated, even a small concentration of positively-charged analyte further induces aggregation of the colloid, and this is reflected in the concomitant increase in SERS EF from the lowest dye concentrations (from $25 \mathrm{nM}$ to $50 \mathrm{nM}$ and $100 \mathrm{nM}$ ). The measured EF keeps increasing up to a maximum of $1.3 \times 10^{6}$ at $200 \mathrm{nM}$, beyond which the colloidal solution collapses/sediments resulting in a sharp drop in SERS signal. It should be noted that partially-aggregated Lee \& Meisel colloids do not exhibit any welldefined aggregate extinction peak and extinction spectra cannot here be used as a proxy for the aggregation state [71].

These results clearly highlight the effect of molecular coverage on colloid aggregation in our system, and can now be linked to the observed dilution effects.

When large dilution factors are used, the strongly non-uniform coverage may result in aggregation for the small number of colloids with the highest coverage, and therefore in a larger SERS EF for those colloids. Since the aggregated colloids are those with the most dyes, the average SERS EF for the entire solution is also increased. For example, from Figure 4.6(A), we see that a $100 \mathrm{nM}$ solution with uniform coverage (i.e. prepared from the HHD method) exhibits an average enhancement of $10^{4}$. The same solution prepared using a $100 \times$ dilution (from $10 \mu \mathrm{M}$ ) will contain many NPs with no adsorbate and many NPs with coverage close to saturation (see Fig. 4.6(D)). The higher local concentration will clearly cause local aggregation of the minority of colloids in the region where the dye is injected, and the EF for these will be of the order of $10^{5}$ (according to Fig. 4.6(A)). The overall SERS signal will be increased by a factor up to 10, depending on the details of the competition between molecular adsorption and mixing dynamics. This explains the factors of 4.5 and 7 observed for NB and R6G in Figures 4.1 and 4.2 and similarly the factors in Figure 4.3.

It should be noted that the trends observed for the concentration induced AEFs measured in Figure 4.6 are specific to the colloids used and will be 
strongly dependent on the intrinsic properties of the colloids themselves. In particular, the relative concentration of colloids to dyes, colloid size, electrolyte concentration, colloid stabilising agent and interaction between the dye and the colloid will all play a role in determining at what concentration the onset of dye-induced aggregation begins. As an example, assuming all other parameters are kept the same, for more concentrated colloids the onset of aggregation would occur at higher dye concentrations because each dye reduces the overall surface charge of the colloids by a smaller amount thus requiring more dyes to cause instability of the colloids. This interpretation is consistent with DLVO theory where each positively charged dye will reduce the total electrostatic repulsion between like-charged colloids. This also implies that similar to the Lee \& Meisel system studied here, for any colloidal solution there should exist a range of analyte concentrations where the SERS EFs are constant (i.e. linear response to analyte concentration) up to a certain limit. Beyond this limit, AEFs will vary with increasing analyte addition, becoming super-linear and deviating from ideal behaviour often sought when using SERS for routine detection.

\subsubsection{Finite Element Modelling of Diffsusion}

In reality the exact distribution of coverage obtained with the LDF method (Figure 4.4(C)) will depend on the details of the kinetics of adsorption vs mixing, and any intermediate situations between the extreme cases of Figures $4.4(\mathrm{C})$ and $(\mathrm{F})$ can in principle be obtained. It is instructive to make this argument more quantitative by modeling the situation on a simple model system as shown in Figures 4.7 and 4.8. Here a cylindrical container of radius $8 \mathrm{~mm}$ and height $2 \mathrm{~cm}$ (i.e. volume of $1 \mathrm{~mL}$ as for a typical sample preparation), is considered, into which the colloidal NPs are uniformly distributed and considered fixed since colloidal diffusion is much slower than dye/molecular diffusion. In reality, the diffusion of the colloids, $D_{\text {col }}$ is set to $10^{-15} \mathrm{~m}^{2} \mathrm{~s}^{-1}$ The approach is then to solve the diffusion equation (Fick's second law) for the analyte, coupled to a simple adsorption rate equation (which takes into account saturation at monolayer coverage). Explicitly, the differential equations are: 


$$
\begin{aligned}
\frac{\partial c}{\partial t} & =D \Delta c-k_{A} c\left(1-\frac{c}{c_{\mathrm{sat}}}\right) \\
\frac{\partial c_{A}}{\partial t} & =k_{A} c\left(1-\frac{c}{c_{\mathrm{sat}}}\right) .
\end{aligned}
$$

$c(\mathbf{r}, t)$ and $c_{A}(\mathbf{r}, t)$ represent the concentration of free and adsorbed analyte, $D$ is the diffusion coefficient of the free analyte, $k_{A}\left[\mathrm{~s}^{-1}\right]$ characterizes the rate of adsorption at low coverage, and $c_{\text {sat }}$ is the concentration at the saturation coverage (which here is set at $500 \mathrm{nM}$ here but this depends on the colloid concentration). The initial condition is set as $c=c_{0}$ inside a small cylindrical volume of $10 \mu \mathrm{L}$ for the $100 \times$ dilution (or $0.5 \mathrm{~mL}$ for a half-half dilution) and $c=0$ elsewhere (and $c_{A}=0$ everywhere). In order to model the convection-driven mixing/stirring, $D$ is set to a value much larger than its normal free-diffusion estimates (for dyes like Rhodamine 6G, diffusion coefficients in water are of the order of $\left.10^{-6} \mathrm{~cm}^{2} / \mathrm{s}\right)$ : here it is adjusted to $D=0.1 \mathrm{~cm}^{2} / \mathrm{s}$, which results in a uniform concentration across the sample ((in the absence of adsorption)) after about $10 \mathrm{~s}$ for the $\times 100$ dilution and about $0.4 \mathrm{~s}$ for the $\times 2$ dilution. These are reasonable values for the physical time it would take for the dyes to disperse in the cuvette when injected with a pipette. The adsorption rate is set to $k_{A}=10 \mathrm{~s}^{-1}$. This results in $95 \%$ adsorption in $0.3 \mathrm{~s}$ which is a slight overestimate from the fluorescence quenching measurements in Section 4.3.3, where the fluorescence was minimised after $1.6 \mathrm{~s}$. Those results are difficult to quantify exactly the percentage of adsorbed dyes after a given time so it is assumed here that the majority of adsorption occurs in sub-second time scales.

The problem is then solved numerically with finite-element modeling in COMSOL, whereby the free and adsorbed analyte concentration across the sample are solved for at each time $t_{i}$. The colloids essentially are fixed throughout the cuvette volume, and act as a sink term whereby freely diffusing species, $c(\mathbf{r}, t)$, are removed from solution through Eq. 4.2. Each "colloid" can remove an upper limit of dyes set at $c_{\text {sat }}$.

From the long-time (typically 111s) spatial distribution of the adsorbed species concentration $c_{A}(\mathbf{r})$, the statistical properties of the distribution of 
adsorbed molecular coverage across the sample, i.e. average, maximum, and minimum coverages, or the histogram of coverages, can be computed.

The coverage of colloids can be found from:

$$
N_{\mathrm{cov}}=\frac{c_{\mathrm{dye}}}{c_{\mathrm{col}} A_{\mathrm{col}}}
$$

where $c_{\text {dye }}$ is the adsorbed dye concentration, $c_{\mathrm{NP}}$ is the colloid concentration and $A_{\text {col }}$ is the surface area of a single spherical colloid. Thus for a $30 \mathrm{~nm}$ radius solution of colloids at a concentration of 20 pM (i.e. Lee \& Meisel colloids at 4 times dilution) a dye coverage of 1 (i.e. 1 dye per $\mathrm{nm}^{2}$ ) corresponds to a dye concentration of $\sim 500 \mathrm{nM}$, or approximately 28250 dyes per colloid.

A series of dilution situations were modelled to give a detailed description of the mechanisms at play governing the nanoparticle coverage. Four "real" dilution factors that would typically be used in the lab were tested; $\times 100, \times 50$, $\times 10$ and $\times 2$ (half-half) dilutions of dyes into a $1 \mathrm{~mL}$ solution of colloids, all yielding the same final total dye concentration. As above, the diffusion rate and adsoprtion rate were fixed for each dilution factor and the colloid saturation was set to $500 \mathrm{nM}$ to match that of Lee and Meisel colloids. Each dilution factor was modeled using a final dye concentration of $100 \mathrm{nM}$ and $10 \mathrm{nM}$ to investigate the dependence of the diffusion vs adsorption phenomenon on final dye concentration. The results of these models are shown in Figures 4.7 and 4.8 .

The histograms obtained from the modeling series yield a wealth of interesting information. Most importantly, the simulations reveal that indeed there is a significant dispersion of molecular coverages across the solution when large dilution factors are used. For large dilution factors $(100 \times$ and $50 \times)$, the predicted histograms of molecular coverage exhibit a spectacular non-uniformity, with maximum coverages as large as 5 times the average. From the spatial distributions of concentration across the cuvette it is clear that the adsorption reaction acts to limit the diffusion distance of the dyes; even after $100 \mathrm{~s}$ the majority of the molecules are concentrated in the center of the cuvette with the top and bottom regions containing essentially no molecules. As the dilution ratio is reduced down to $10 \times$ and finally $2 \times$, is is clear that the distribution 

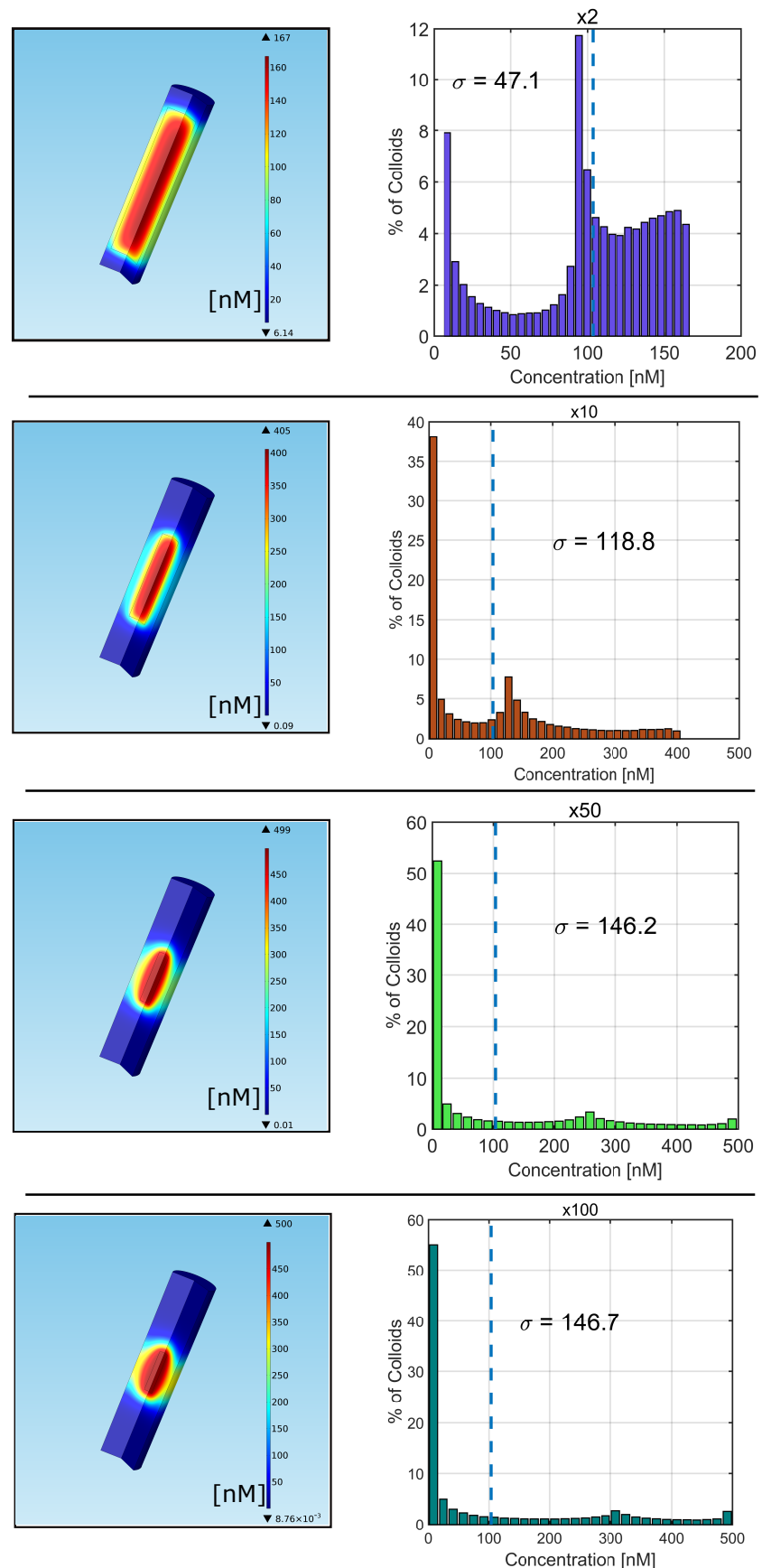

Figure 4.7: Theoretical predictions from COMSOL of the spatial distribution of adsorbed dyes for a x2, x10, x50 and x100 dilution for final dye concentration of $100 \mathrm{nM}$. The left panels show the geometry of the model and the map of concentrations as output from COMSOL, while the histograms on the right show the corresponding percentage of colloids with a given concentration of adsorbed dyes. The blue dashed line is the final average dye coverage (as expected, $100 \mathrm{nM}$ ) and the standard deviation from this mean coverage is shown. The saturation coverage is set at $500 \mathrm{nM}$ to match that of Lee and Meisel colloids. 

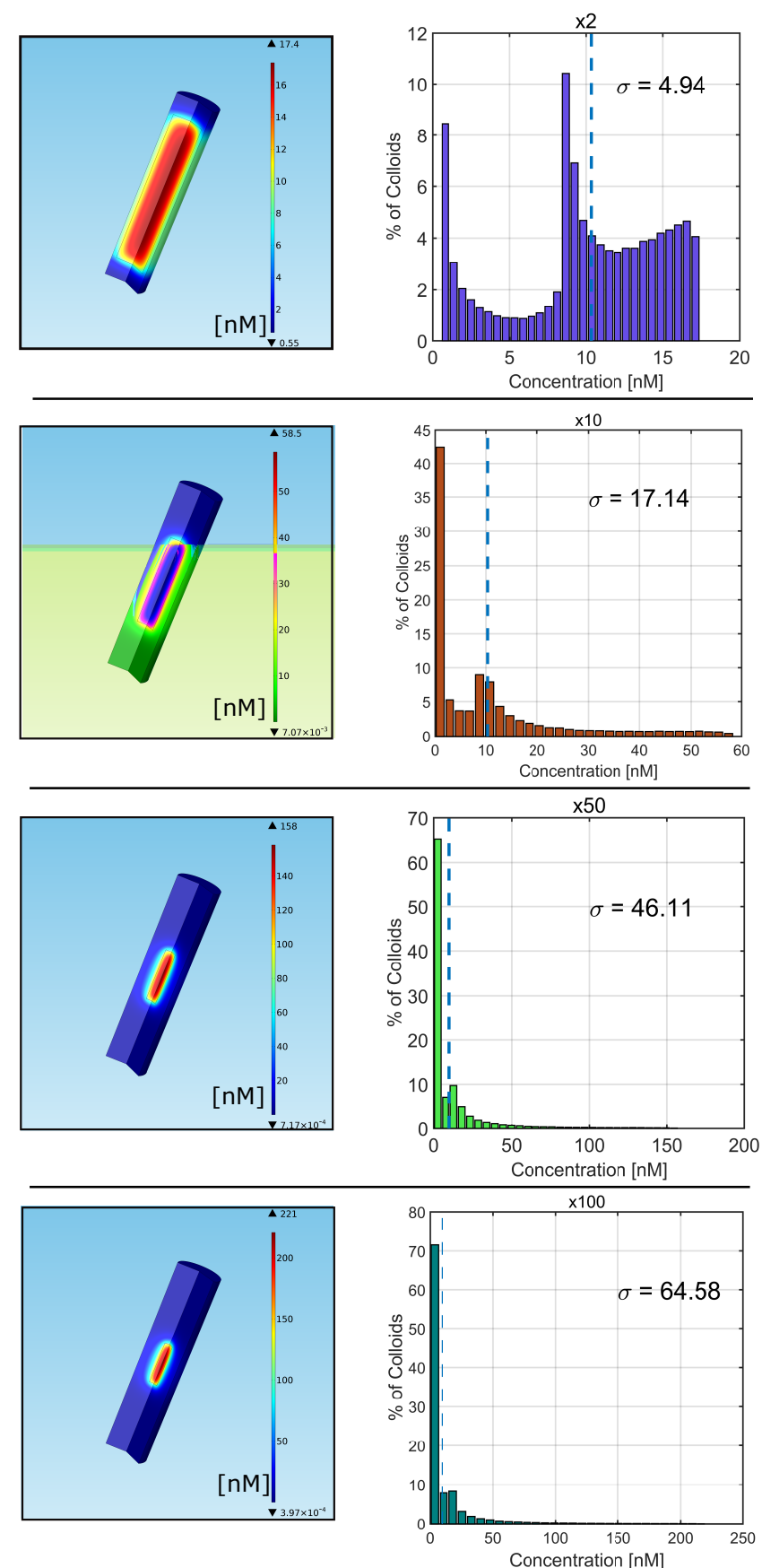

Figure 4.8: Theoretical predictions from COMSOL of the spatial distribution of adsorbed dyes for a x2, x10, x50 and x100 dilution for final dye concentration of $10 \mathrm{nM}$. The left panels show the geometry of the model and the map of concentrations as output from COMSOL, while the histograms on the right show the corresponding percentage of colloids with a given concentration of adsorbed dyes. The blue dashed line is the final average dye coverage (as expected, $10 \mathrm{nM}$ ) and the standard deviation from this mean coverage is shown. The saturation coverage is set at $500 \mathrm{nM}$ to match that of Lee and Meisel colloids. 
of coverages reduces correspondingly, as indicated by the standard deviations from the mean coverage in each histogram.

Also evident is the dependence of the distribution of coverages on the final dye concentration, where the coverages for $10 \mathrm{nM}$ dye (Figure 4.8) show a significantly less deviation from the average coverage than those for $100 \mathrm{nM}$ dye in Figure 4.7, confirming the observations in Section 4.3.1 where the signal discrepancies in average enhancements between HHD and LDF methods were lower when the dye concentration was reduced by a factor of 10 . Physically this can be interpreted as the adsoprtion occurring slower due to the lower concentration gradients in the solution, allowing dyes to diffuse further through the cuvette volume. Nevertheless the effects of dilution factor are still important even as the dye concentration is lowered, further highlighting the recommendation of half-half dilutions to be used.

While the illustration is fictitious in the sense that the colloids in reality are not fixed and would diffuse around the cuvette, the spatial distributions vividly highlight the effect of adsorption being present on diffusion dynamics. The coverage histograms support this picture showing that some colloids may not have any dyes adsorbed at all if large dilution factors are used. Moreover, the results illustrate how in many experimental conditions, the large nonuniformity in coverages may be completely hidden in the signals measured. For example, (assuming no dye-induced aggregation of colloids is present) average SERS signals, whereby the entire colloidal solution is sampled and the signal is the average of many spectra, would show exactly that of the average coverage calculated from the histograms. While this may be desirable for measurements where only the average SERS signal is of interest, each individual spectrum may show large intensity variations due to there being at times some colloids with no dyes and some with many in the scattering volume. These nonuniformities would then play a role in time-dependent and single-molecule measurements, where the signal of each individual spectrum is monitored.

In the case of dye-induced aggregation, the COMSOL models support the proposed interpretation that large dilutions will cause the colloids whose dyecoverage is much larger to aggregate and thus the solution will exhibit larger SERS signals. 
Interestingly, even a half-half dilution results in a non-negligible spread of molecular coverage, but this would strongly depend on the exact details of the mixing procedure. Because the adsoprtion rate is so fast compared to the diffusion, even the ideal half-half method would not alleviate the problem completely but it goes a long way to addressing the non-uniformities usually present.

\subsubsection{SEM Evidence for Dilution Effects}

An obvious step towards further evidencing the effects of sample preparation on the aggregation state of nanoparticles is to directly image samples via SEM or TEM. In theory, if the hypothesis presented above is correct, differences in the size and number of nanoparticle clusters present across samples prepared by the two dilution methods should support these claims. Given the evidence from SERS, there should be a clear increase in the average number of clusters in the samples prepared by the large dilution factor due to the aforementioned local aggregation induced by dye molecules. SEM was the preferred method of imaging due to the large areas that can be quickly imaged. This gives a much better overview of the average state of the sample whereas in TEM it can be cumbersome to image a large number of particles.

In practice however this approach proves more difficult to pin down for a number of reasons. Figure 4.9 presents SEM images for three colloidal samples deposited on Si wafers: (a) a reference solution of $60 \mathrm{~nm}$ Ag Nanocomposix colloids diluted by a factor of four to a final $\mathrm{KCl}$ concentration of $0.5 \mathrm{mM}$, (b) a $100 \mathrm{nM}$ R6G sample of the same colloids (i.e. $0.5 \mathrm{mM} \mathrm{KCl}$ final concentration) prepared by the half-half method and (c) 100 nM R6G sample prepared by the large dilution factor. It is important to note here that samples were prepared in a specific fashion to try and "capture" the true state of the nanoparticles in solution; this consists of coating the Si wafers onto which particles are dried with a postively charged layer that will attract the particles and essentially "freeze" them as they were in solution instead of allowing them to dry and possibly induce aggregation. This method was shown to work in previous studies in our group [74] and the preparation procedure is revisited briefly here. 
1. A drop of $1 \%$ weight poly-l-lysine aqueous solution was added to cleaned Si wafers and allowed to sit for $\sim 5$ minutes before rinsing off with distilled water.

2. $10 \mu \mathrm{L}$ of nanoparticle solution was then drop-cast onto the Si wafer and washed off after $\sim 2$ minutes.

3. Wafers were then placed in a desiccator over night.

4. Before imaging, samples were sputter coated with $4 \mathrm{~nm}$ of platinum to improve sample conductivity for imaging.

It is also important to note that the colloids used here are the $60 \mathrm{~nm} \mathrm{Ag}$ spheres purchased from Nanocomposix as described in Chapter 4. The reason for using these colloids as opposed to the Lee and Meisel particles used in the SERS studies of the previous sections is the vast improvement in monodispersity in the Nanocomposix samples. These colloids have been extensively optimised and purified and show a much better size dependence than Lee and Meisel. This is evidenced from both UV-Vis spectra, where Nanocomposix show a much sharper plasmon resonance than Lee and Meisel colloids and SEM/TEM images where Nanocomposix colloids have an excellent size distribution of $60 \mathrm{~nm}$ spheres whereas Lee and Meisel colloids clearly contain many other particle shapes and sizes (Chapter 3). As such it was decided any changes in cluster size/numbers due to dilution effects would be easier to detect using the superior Nanocomposix colloids.

The results from SEM indciate that even in the reference colloidal solution, where no dyes are present and the $\mathrm{KCl}$ concentration is much too low to induce aggregation (from DLVO theory), there still exist a reasonable number of clusters. Were the samples simply allowed to dry on the substrates, one would assume that any aggregation (clusters) present would be a combination of the drying process and the original presence of clusters, thus hindering any interpretation of the results. In this case however, it is safe to assume that the electrostatic attraction provided by the poly-l-lysine should effectively give a snapshot of the particles as they are in solution. As such, Figure 4.9 highlights the difficulty in using a "dried" version of nanoparticles, as in SEM, to determine the presence of clusters created by dilution. The images presented are 


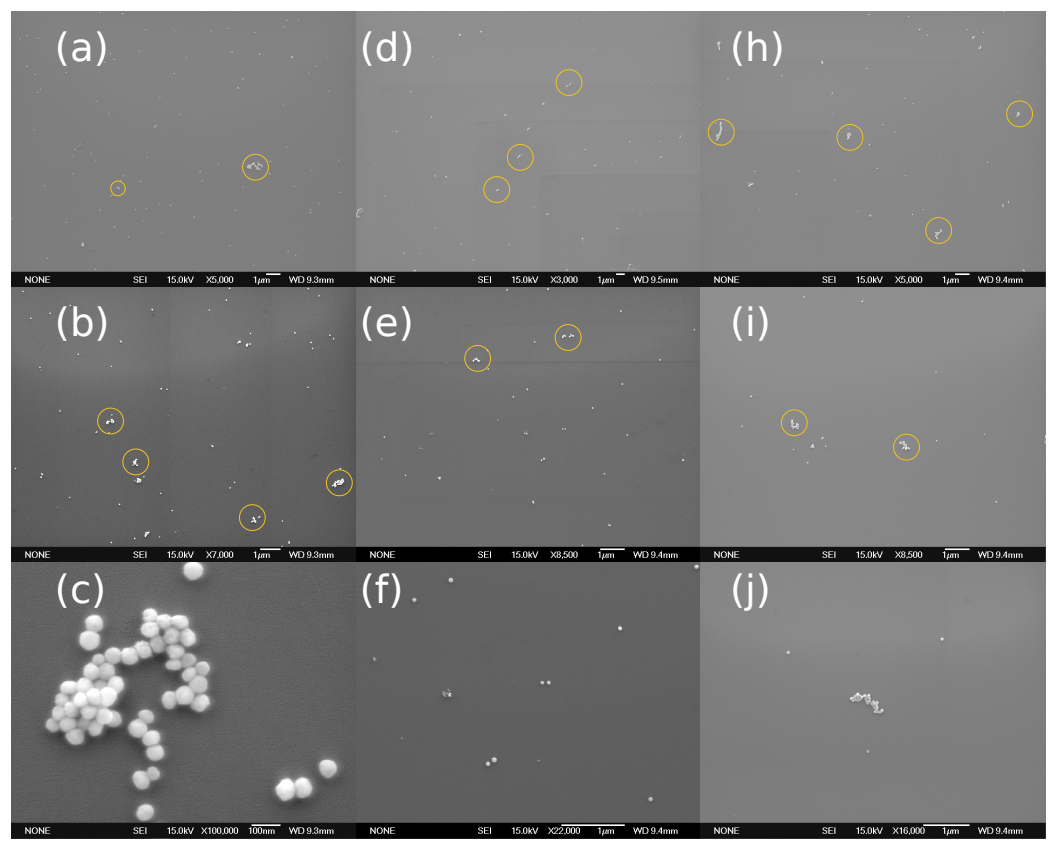

Figure 4.9: SEM images of $60 \mathrm{~nm}$ Ag colloids prepared by different dilution methods and deposited on poly-l-lysine coated Si wafers. (a-c) Reference samples containing 1:1 ratios of colloids to $\mathrm{KCl}(0.5 \mathrm{mM}$ final concentration). (d-f) Colloids with $100 \mathrm{nM}$ R6G final concentration prepared by the half-half dilution method. (h-j) Colloids with $100 \mathrm{nM}$ R6G final concentration prepared by the large dilution method. Three representative areas at three magnifications for each sample are shown. Yellow circles indicate clusters in each sample. 
representative examples of each sample and it would be fruitless to quantify the number of overall clusters present for each dilution method given that the reference sample already contains clusters ranging from 50 to 200 single particles. In all samples, there are a large number of single isolated particles along with dimers, trimers and other larger species. These clusters may be present in the bare colloid solution prior to deposition but they are not apparent in the UV-Vis extinction spectra, where aggregation is normally evidenced by a long wavelength tail developing in the $\sim 600-900 \mathrm{~nm}$ region. Alternatively, clusters could very possibly form during the drying process. It is interesting to note that in the large dilution factor sample (Figure $4.9(\mathrm{~h}-\mathrm{j})$ ) there was an indication that the clusters were larger (a few hundred particles) than those in the reference and HHD samples but they were quite rare. This was not conclusive enough to confirm the proposals using SERS and fluorescence quenching but further work in this area, (i.e. optimising the drying procedure, changing colloid concentration and filtering particles before adding dyes to remove any large clusters in the bare solution) is needed to fully trust the SEM results. Nevertheless the results further strengthen the point that SERS in itself is far more sensitive than any other available technique for characterizing the subtle substrate properties at play in metallic colloids.

\subsubsection{Effects on Single Molecule SERS}

While this chapter has focused so far on average SERS EFs, it is clear that the strong non-uniformity in molecular coverage resulting from large-factor dilutions should also have a profound effect on the statistics of SERS signals. This should particularly affect single-molecule SERS studies where coverage estimates and statistics of signals play a crucial role in identifying the correct conditions for single-molecule detection. The consequences of the findings for single molecule SERS (SM-SERS) are therefore now addressed.

Firstly, it is clear that the average molecular coverage has little meaning in the case of samples prepared by the LDF method, since a small number of NPs will have a much larger number of molecules. The original studies of SMSERS $[2,3,104]$ in fact relied on such estimates to justify the single molecule nature of the SERS signals. The targeted concentration was adjusted such 
that on average, less than one molecule (say 0.5) was measured at a given time. SERS events were then rare occurrences, but they were attributed to SM-SERS. It is now clear from this study that if the samples are prepared using a $100 \times$ dye-dilution (or similar large dilutions), then a small fraction of nanoparticles could in fact have a much larger coverage than average (0.5), with up to say 10 molecules per NPs. The rare SERS events would then most likely correspond to those high-coverage particles rather than to SM-SERS.

In fact, as discussed in Ref. [86], there were many additional potential problems with the low-concentration approach, which prompted the development of bi-analyte SERS [86] as a more convincing and statistically robust method of studying SM-SERS $[105,106]$. Within this framework, two analytes with comparable SERS cross-section but a distinguishable SERS spectrum, are used to experimentally determine the nature of the SERS events, either predominantly SM-SERS or multi-molecule SERS [32,107], without any assumptions on the coverage. Ideal implementations of the method make use of two isotopically different versions of the same molecule. Central to the interpretations of any bi-analyte SERS experiment is the implicit assumption that the distribution of molecules across the sample is the same (even if potentially non-uniform) for both bi-analyte partners. This aspect was implicit in the original bi-analyte work [86,107], and in the light of the current study, needs to be clearly re-emphasized.

It is demonstrated explicitly in Figures 4.10, 4.11 and 4.12 how the wrong conclusions about the SM-SERS regime could be attained because of the dilution problems evidenced in this work. $\mathrm{Bi}$-anlayte single-molecule samples consisted of $10 \mathrm{nM}$ each of a modified rhodamine $6 \mathrm{G}$ and its isotopicallysubstituted partner with 4 deuterium atoms (as studied in detail in [91]) mixed with pre-aggregated Lee and Meisel silver colloids. Explicitly, colloids were mixed with $20 \mathrm{mM} \mathrm{KCl}$ in a 1:1 ratio to achieve a final $\mathrm{KCl}$ concentration o of $10 \mathrm{mM}$. The solution was left to aggregate for at least one hour before analytes were added and again left to sit for 1 hour before measuring. To obtain sufficient statistics, 1500 spectra were acquired for each sample with an integration time of 0.1 s. 1500 dark spectra were also acquired under the same conditions that were then averaged and subtracted from each individual SERS spectrum.

Figure 4.10 shows a typical bi-analyte SERS analysis, where a scatter plot 
of the relative contribution of each dye to the individual SERS spectra are plotted. The underlying principles of the bi-analyte technique with isotopic substitution were discussed in Chapter 3. As a brief reminder to the reader, Raman spectra of the isotopic partners can be distinguished from each other because Rhodamine $6 \mathrm{M}$ exhibits a strong Raman peak at $612 \mathrm{~cm}^{-1}$ while for the deuterated version (d-R6M), the peak is shifted to $600 \mathrm{~cm}^{-1}$. This allows one to easily identify three types of SERS events; (1) mixed events, where both peaks are present, (2) events solely due to R6M and (3) events solely due to d-R6M. Events of type (1) will be located about the centerline of the scatter plot, type (2) will have only $\mathrm{x}$-axis contribution and type (3) only $\mathrm{y}^{-}$ axis contribution. This method provides a powerful way to quickly assess if a sample is in the multi-molecule or single-molecule regime. Spectra of interest, that may be single-molecule candidates, can then be identified and plotted individually to assess their single-molecule nature. The results presented below focus only on the $612 \mathrm{~cm}^{-1}$ and $600 \mathrm{~cm}^{-1}$ peaks of the bi-analyte partners for analysis but the same observations are found for the $1500 \mathrm{~cm}^{-1}$ region where both dyes can also be distinguished from each other.

As can be seen in Figure 4.10 (a), most events are mixed, which correspond to the multi-molecule regime, as expected for this system at this dye concentration $(10 \mathrm{nM})$ with very few events located only on either the $\mathrm{x}$-axis or $\mathrm{y}$-axis. In this first instance, both bi-analyte partners were mixed together at $1 \mu \mathrm{M}$, then diluted to $20 \mathrm{nM}$, and then diluted by a factor of 2 with the final colloid solution, i.e. the HHD method recommended in this work was used to avoid dilution problems. Two representative Raman spectra from the center-line of events (highlighted in the scatter plot by red and green circles) are then plotted individually (1-2) (red curves) along with the corresponding fits to a mixture of R6M and d-R6M spectra. As can be seen, both spectra show almost equal contributions of both dyes indicating they arise from multi-molecule events.

In most previous works $[86,107,108]$ it is likely, (or not specified) that large dilution factors were used, and the results obtained from an equivalent $100 \times$ dilution are therefore shown in Figure 4.11. As expected from the previous discussion, a small number of NPs exhibit much higher coverage (for both molecules) and this is reflected in the more intense events that are observed (the majority of events in (b) are significantly larger than those in (a) indicative of dye-induced aggregation), and in the stronger concentration of events around 

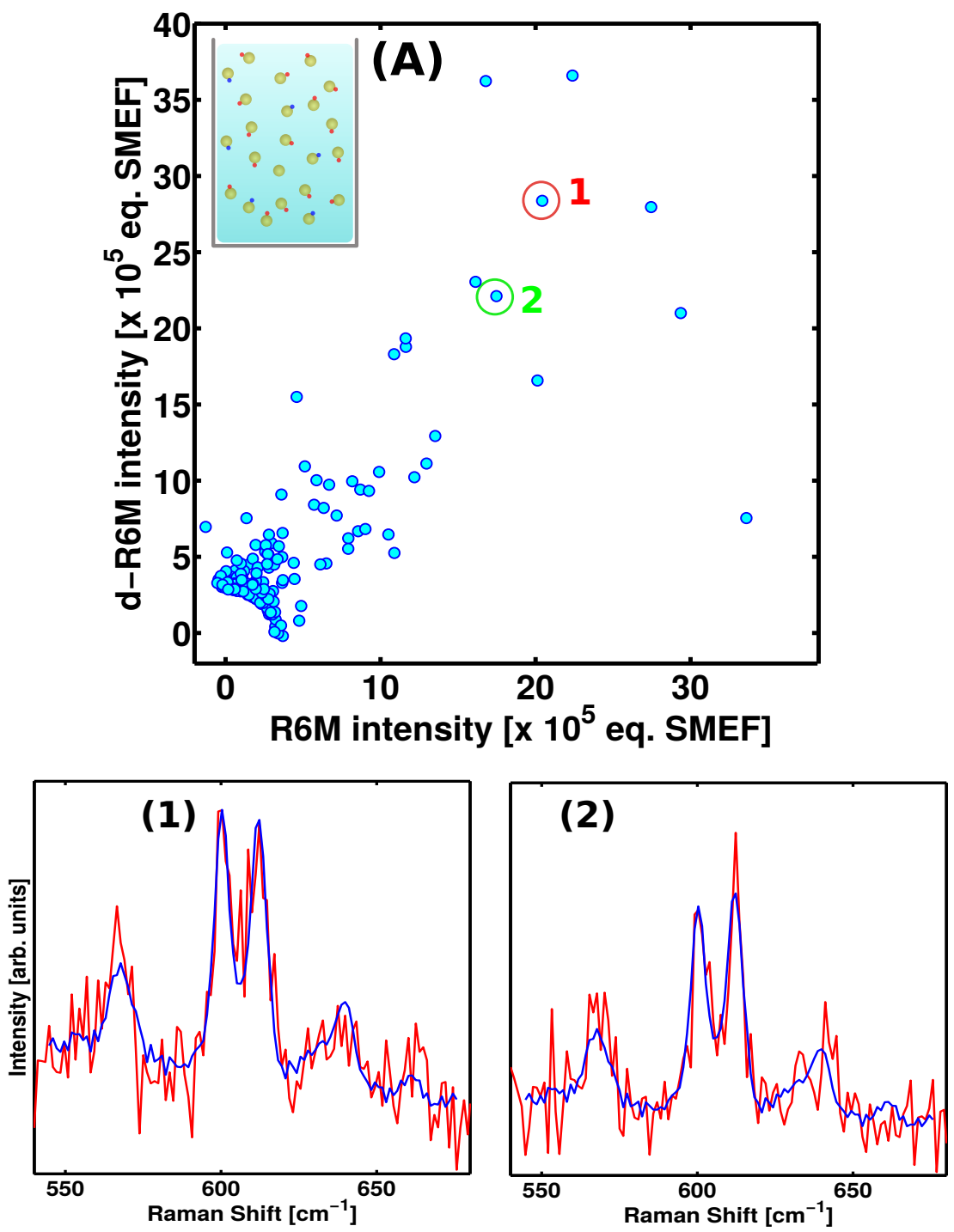

Figure 4.10: The effect of different sample dilution procedures on the statistics of bi-analyte single molecule SERS spectra using isotopic partners R6M and d-R6M at $10 \mathrm{nM}$. Scatter plots of the intensity of the $612 \mathrm{~cm}^{-1}$ peak of R6M ( $x$-axis) and the $600 \mathrm{~cm}^{-1}$ peak of d-R6M ( $y$-axis) for samples where dyes are pre-mixed together and then diluted with the HHD method. A schematic showing the expected outcome of the dilution on nanoparticle coverage is shown as an inset. Ag colloid are pre-mixed with $20 \mathrm{mM} \mathrm{KCl}$ for pre-aggregation. Representative spectra (1-2) are shown at the bottom along with the corresponding fits to a mixture R6M and d-R6M spectra. 

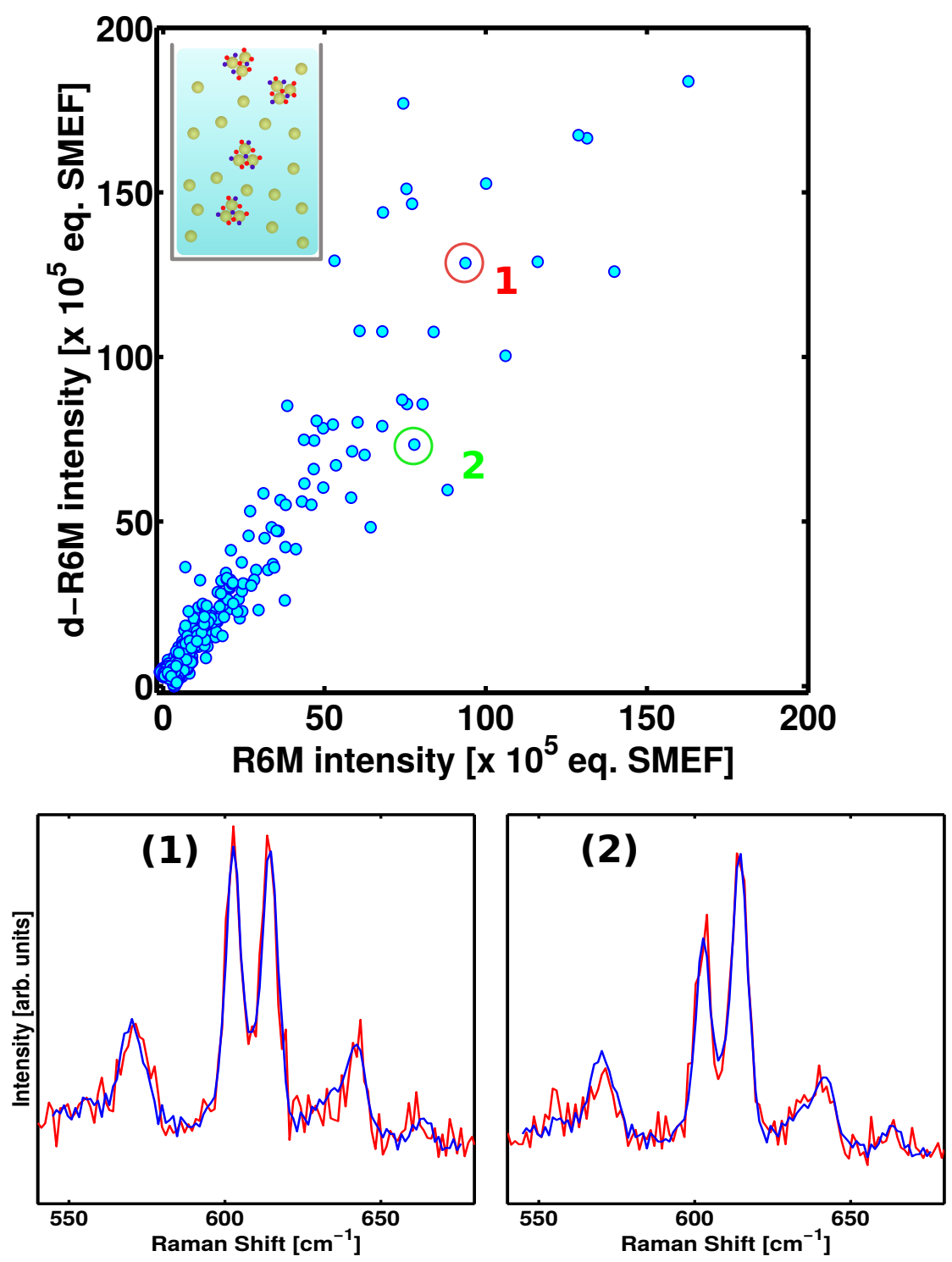

Figure 4.11: The effect of different sample dilution procedures on the statistics of bi-analyte single molecule SERS spectra using isotopic partners R6M and d-R6M at $10 \mathrm{nM}$. Scatter plots of the intensity of the $612 \mathrm{~cm}^{-1}$ peak of R6M ( $x$-axis) and the $600 \mathrm{~cm}^{-1}$ peak of d-R6M ( $y$-axis) for samples where dyes are pre-mixed together and then diluted using the LDF method with $100 \times$ dilution. A schematic showing the expected outcome of the dilution is shown as an inset. Ag colloids are pre-mixed with $20 \mathrm{mM} \mathrm{KCl}$ for pre-aggregation. Representative spectra (1-2) are shown at the bottom along with the corresponding fits to a mixture R6M and d-R6M spectra. 

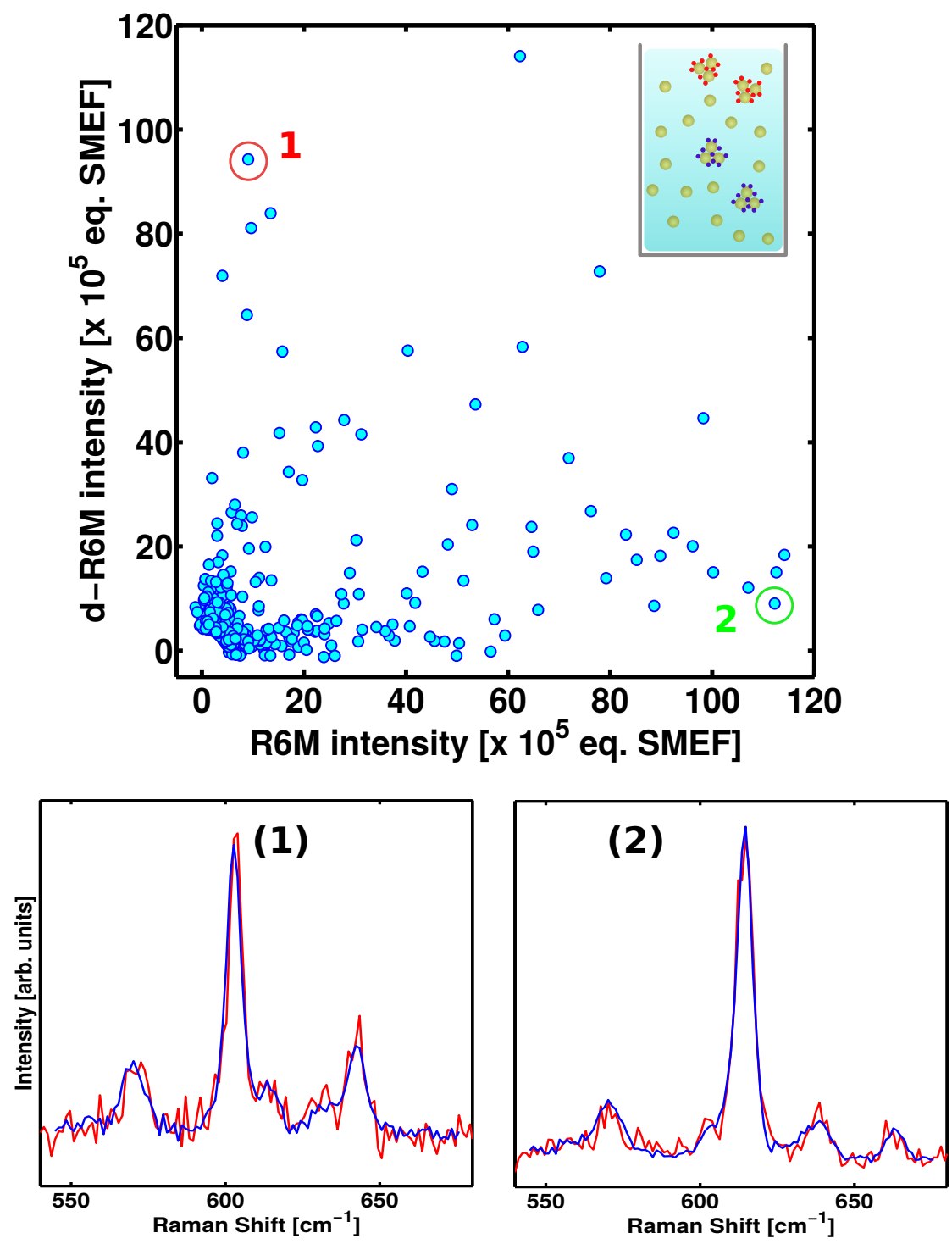

Figure 4.12: The effect of different sample dilution procedures on the statistics of bi-analyte single molecule SERS spectra using isotopic partners R6M and d-R6M at $10 \mathrm{nM}$. Scatter plots of the intensity of the $612 \mathrm{~cm}^{-1}$ peak of R6M ( $x$-axis) and the $600 \mathrm{~cm}^{-1}$ peak of d-R6M ( $y$-axis) for samples where dyes are added sequentially to the NP solution using the LDF method with $100 \times$ dilution. A schematic showing the expected outcome of the dilution is shown as an inset. Ag colloids are pre-mixed with $20 \mathrm{mM} \mathrm{KCl}$ for pre-aggregation. Representative spectra (1-2) are shown at the bottom along with the corresponding fits to a mixture R6M and d-R6M spectra. 
the 50\%-50\% line, indicating an even more pronounced multi-molecule regime than obtained from the HHD method in Figure 4.10. Two representative Raman spectra are from the centerline (highlighted in the scatter plot by red and green circles) are again plotted individually (1-2) (red curves) along with the corresponding fits to a mixture of R6M and d-R6M spectra. Again both spectra show almost equal contributions of both dyes indicating they arise from multi-molecule events. Note the improved signal to noise ratio in these events, due to the larger absolute intensities of the spectra.

An even more spectacular consequence of the dilution problem is evidenced in Figure 4.12, where the bi-analyte partners were diluted $100 \times$ sequentially into the colloid solution, instead of being pre-mixed together as in Figures 4.10 and 4.11. In such a case, those NPs with a larger than average coverage of one dye are not the same as those for the other dye. The statistics of events then become characteristic of the single-molecule regime, with several pure events of one dye or the other. These seemingly SM-SERS events in reality correspond to NPs with a large coverage of one dye and not the other as is evident now from the proposed and validated interpretation of diffusion-adsorption competition. Thus in view of this new information, the spectra in Figures 4.10 and 4.11 are not SM-SERS events, yet from the point of view of the statistical analysis used in the bi-analyte approach they show up as unequivocal single-molecule events. The immediate appearance of these SM-like statistics from simply altering the dilution procedure is concrete evidence for a weakness of the bi-analyte technique that is not immune to errors from sample non-uniformity. The dilution procedure therefore here introduces artifacts that lead to an entirely wrong conclusion regarding SM-SERS. This interpretation is again supported by the observed larger intensities of events in Figure 4.12 as compared to Figures 4.10 indicating dye induced aggregation resulting from non-uniform colloid coverage.

These observations clearly suggest that, in the context of SM-SERS, only bi-analyte experiments where dyes are pre-mixed before dilution into colloids can be trusted. It is difficult to assess whether such problems have affected previous studies, since such preparation details are not always specified [89]. Figure 4.10, 4.11 and 4.12 focus on demonstrating the risk of erroneous claims of SM-SERS detection if the bi-analyte partners are not pre-mixed together and the LDF method is used. From the results of this study, it should also be 
clear that even if the bi-analyte partners are pre-mixed prior to dilution with colloids, the HHD method should be strongly preferred to avoid non-uniform coverage across the sample and irreproducibility. Indeed, large variations in surface coverage may result in part of the sample being in the single-molecule regime and part in the multi-molecule regime, therefore blurring and confusing the statistical analysis on SM-SERS intensities. It is therefore expected that the adoption of the HHD method for SM-SERS studies will result in much stronger conclusions and a much clearer transition between the single-molecule and multi-molecule regime. Further work is needed in this direction in terms of optimising the aggregation procedure to allow single molecule detection to be a reproducible technique. Specifically, the role of dye induced aggregation clearly needs to be minimised so as the single-molecule properties of the substrate can be tailored independently of the analyte used. It is plausible that many of the single-molecule studies performed in the past may have benefited from dye-induced formation of hot spots rather than electrolyte induced, which has obvious disadvantages.

\subsection{Conclusion}

It has clearly been demonstrated how apparently similar dilution/preparation methods of colloidal SERS solutions can result in drastically different properties, in terms of both average signals and their statistics in the context of single molecule detection. Large dilution factors of the analytes into the NPs solution create a large variation in molecular coverage per NPs across the sample. This effect is naturally interpreted as a consequence of the competition between the typically fast analyte adsorption onto NPs and the comparatively slow diffusion/mixing process, even when driven by forced convection (shaking/stirring). As such, this effect is not expected to be general, and will only affect experiments where the analyte adsorption is much faster than the mixing time. It is possible that non-cationic analytes, which are less attracted to the negatively-charged colloids, may not be affected by this problem because they adsorb more slowly, but this should be assessed on a case-by-case basis. Nevertheless, the examples presented in this study clearly show that this effect is indeed important for analytes (such as Rhodamine 6G and Nile Blue) and colloidal solutions (Lee \& Meisel Ag colloids) that are commonly used and 
studied. It may therefore have had a dramatic (but unnoticed) impact on the interpretations of many previous SERS studies. We have shown in particular how certain sample preparation methods can lead to extreme misinterpretations of the nature of single molecule SERS spectra when using the bi-analyte technique. Moreover, although the focus here has been on the analyte dilution step only, similar problems were observed when mixing the colloidal solution with salts such as $\mathrm{KCl}$ using large-dilution factors (for example $100 \mathrm{mM} \mathrm{KCl}$ diluted $10 \times)$. Similar interpretations as presented here apply in such a case and such preparation procedures should be avoided. It is important also to note that the LDF method could be affected by a number of factors that cannot be controlled accurately: how the low volume of dye is injected into the solution, whether mechanical stirring is applied, etc...

These findings highlight the necessity for authors to specify clearly the exact method by which samples were prepared, particularly in the case of colloidal solutions. Perhaps more importantly, most of these problems can be largely avoided by simply using low-dilution factors, i.e. the half/half dilution method, which therefore is recommend as the gold standard for any SERS experiments involving colloidal solutions. It is believed the results provide crucial insight into a simple but often overlooked aspect of SERS experiments and shed light on yet another common source of error in the SERS literature. The conclusions of this work would also extend naturally to dilutions in any chemical systems where a fast local phenomenon such as adsorption may compete with the diffusion/mixing process and may therefore have implications well beyond SERS.

In addition to the implications of the results for dilution experiments, the findings illustrate the extreme sensitivity of SERS as a technique to probe the local and average properties of solutions. It is unlikely the non-uniformity evidenced would not be apparent in other techniques such as fluorescence or UV-Vis spectroscopy while techniques such as Dynamic Light Scattering and Zeta-Potential have limitations when large variations in particle sizes exist in solution. As such SERS has the unique ability to probe sample characteristics at a level of detail much higher than other commonly used spectroscopic techniques.

As mentioned at the beginning of this chapter, the conclusions with re- 
gards half-half dilutions has been applied in all further work in the thesis. The continued use of large dilution factors would have seriously hindered any attempts at obtaining reproducible results. This is particularly relevant for Chapter 6 where the extreme sensitivities required in referencing would have been impossible if dye induced aggregation described was present. 
4.4. CONCLUSION 


\section{Chapter 5}

\section{Integrating Spheres - Theory and Experimental Setup}

In this chapter, the underlying theory of light propagation in diffuse reflecting integrating spheres is presented. The parameters governing the working of an integrating sphere are discussed along with the relevance of such an apparatus to the measurements of dyes and nanoparticles in Chapter 6. In particular, a novel setup consisting of a centrally mounted sample cuvette integrating sphere (CMCIS) is presented along with the calibration and characterisation of this setup.

\subsection{The Theory of Integrating Spheres}

In principle the integrating sphere, or integrating cavity, is an extremely powerful apparatus whose main function is to uniformity distribute light within the cavity through diffuse reflections from the sphere surface. This process removes any angular information contained in the original light source and leads to a uniform distribution of photons inside the sphere after a number of reflections. Integrating spheres are a powerful method for measuring any optical process where the angular dependence of the source, detector or both is problematic. The most natural, and original application of integrating spheres is in photometry and radiometry [109], where the entire flux emitted from a 
light source can be measured over the full $4 \pi$ solid angle of emission. Such a measurement would normally require a goniometer to sweep through all possible angles and integrate the flux measured from the lamp. By placing the lamp inside a diffusely reflecting cavity, a detector placed at a position on the sphere wall will automatically collect the entire flux emitted by the source over all angles because the sphere surface is uniformly bright. Other applications include measuring total reflectance and transmission of diffuse or scattering materials [110], calculating the absolute fluorescence and photoluminescence quantum yields of materials $[111,112]$ and obtaining the total cross-section of an optical process (for example the total Raman cross section for a given mode of a molecule).

Integrating spheres have a number of interesting and advantageous properties other than the uniform distribution of photons; for the measurements performed in Chapter 6, an integrating sphere is employed for absorption spectroscopy, for which the properties of interest are:

1. For an input light source, the photon flux inside the sphere compared to the input flux is increased, leading to an increased effective path length in the sphere.

2. If a scattering sample is placed within the sphere, all the scattered light is sent to the detector, meaning that absorption spectroscopy can be performed that is independent of the scattering properties of the sample.

Point 1 will be addressed below while point two will become relevant in Chapter 6.

\subsubsection{Reflectivity - Lambertian Surfaces}

The principle of the integrating sphere for diffuse reflectance rests on the fact that the surface of the cavity is a Lambertian surface. The idea of a Lambertian surface is illustrated in Figure 5.1 where three types of reflections from a surface are shown. A specular reflection (Figure 5.1 (a)), as is treated in rayoptics obeys the law of reflection whereby an incident ray with angle $\theta_{i}$ with 
respect to the surface normal is reflected at an angle $\theta_{r}$, where $\theta_{r}=\theta_{i}$. In (b) the ray reflects from a rough, partially diffuse surface whereby the angle of the reflected ray does not necessarily equal the angle of reflection as in (a). This configuration represents many real world surfaces that are neither perfectly smooth nor perfectly diffuse. In (c) the ray undergoes reflection from a matte, or Lambertian, surface, which scatters light in all directions. A Lambertian surface is one that obeys Lamberts cosine law where the intensity of scattered light is proportional to the cosine of the angle between the incident and scattered ray. One consequence of this property is that the brightness, or radiance, of a Lambertian surface is independent of the viewing angle of the observer.

(a) Specular Reflection

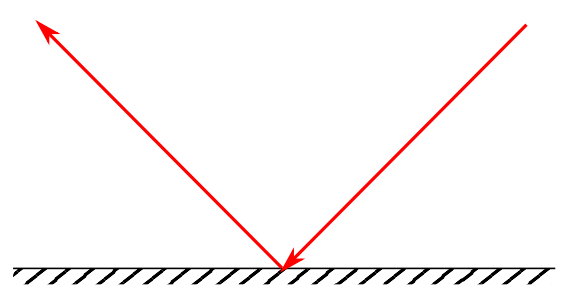

(b) Diffuse Reflection

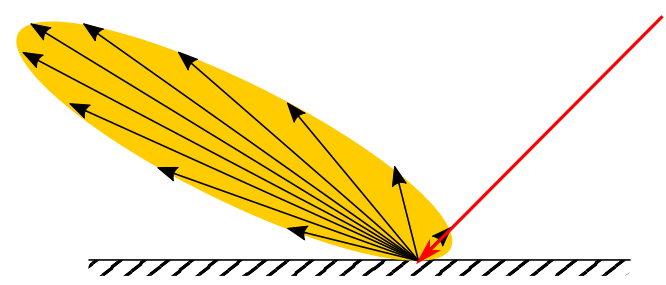

(C) Lambertian Reflection

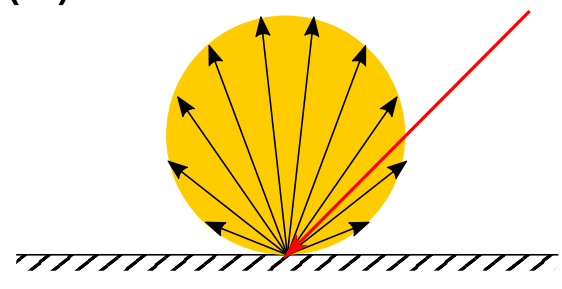

Figure 5.1: Illustrations of the different types of reflections a ray undergoes when it is incident on a surface. (a) Standard specular reflection, (b) a partially diffuse reflection from a rough surface and (c) diffuse reflection from a Lambertian surface.

To understand properly the function of a Lambertian surface, it is best to think in terms of radiance, rather than intensity emitted by the surface. 
The radiance of a surface is defined as the power (or here number of photons) emitted by a unit area into a unit solid angle $\left[\mathrm{W} \mathrm{m}^{-2} \mathrm{sr}^{-1}\right]$. Figure 5.2 (a) illustrates a Lambertian surface that obeys the cosine law [113] of reflected intensity vs angle with respect to the normal. For light falling on the surface at normal incidence, the maximum intensity scattered will be at $\theta=0(\cos (0)=$ 1) and drops off to zero at $\theta=90^{\circ}$. Thus the intensity emitted is a function of angle. However the projected area seen by an observer also however follows a cosine dependence, as shown in Figure 5.2 (b), which compensates for the cosine dependence of the intensity, illustrating that for an observer at any point, the number of photons emitted per unit area and solid angle is the same, i.e. radiance, for a perfect Lambertian surface is independent of the viewing angle of the observer. In the context of an integrating sphere with a Lambertian surface, it follows that the observer, i.e. the detection fiber, sees the same photon flux irrespective of where on the sphere surface it is collecting from. As the light detected by the fiber has undergone multiple Lambertian reflections before reaching the fiber (explained in the following section), one can easily see how the detected light is then independent of the original path travelled, removing any angular dependence the original incident beam may have had.
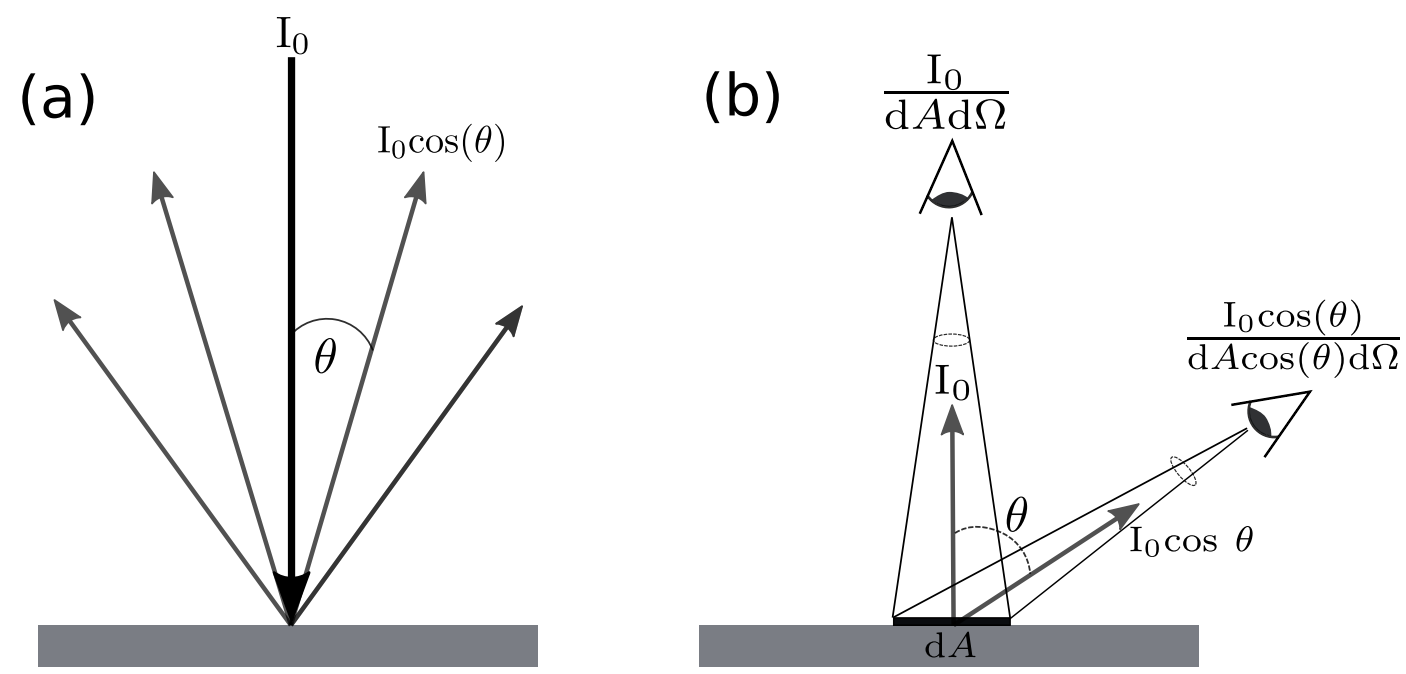

Figure 5.2: Reflections from a Lambertian surface (a) The reflected intensity follows Lamberts cosine law. (b) The area projected from the surface as viewed by an observer also follows a cosine dependence illustrating that the radiance from the surface is independent of viewing angle. 


\subsubsection{Sphere M-Factor}

A simple depiction of an integrating sphere with an entrance and exit port is depicted in Figure 5.3. Much of the theory of integrating spheres has been discussed in detail in References [114-116] but the most important parameters characterising an integrating sphere are:

- surface reflectivity: $\rho$

- port fraction, $f$, the ratio of the total port areas to the sphere surface area: $f=\sum A_{\text {port }} / A_{\text {sphere }}$

- sphere radius: $r_{s}$

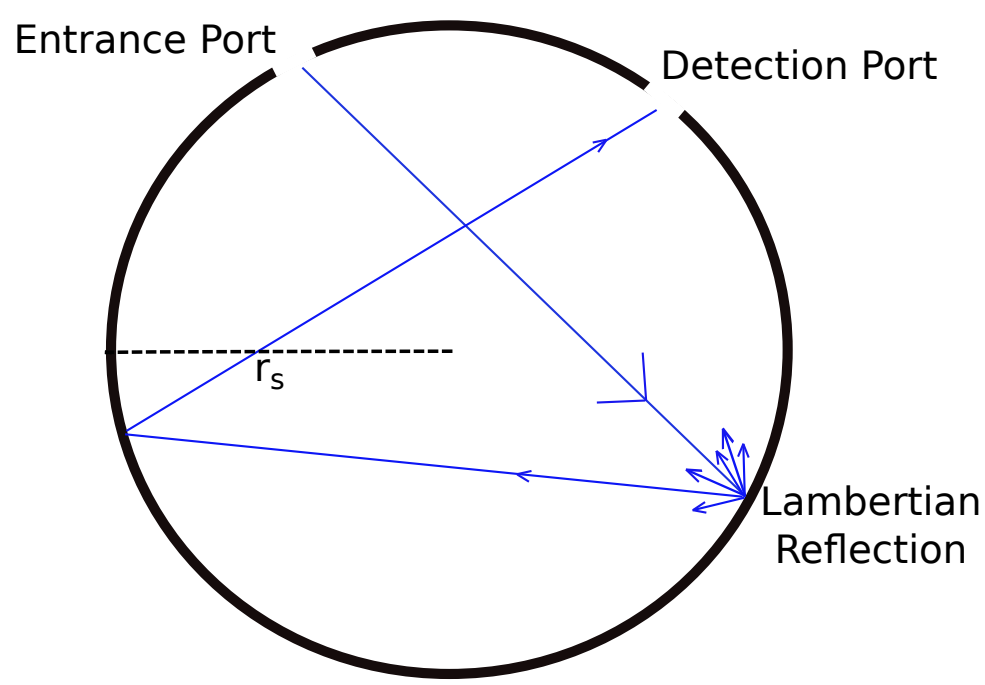

Figure 5.3: A typical integrating sphere with an entrance port and detection port. The path of a single ray that undergoes reflections within the sphere is shown for illustration.

The probability for a ray to reflect off the sphere surface is by definition given by $\rho$ and, upon diffuse reflection, the probability to hit the surface rather than a port is $1-f$. The chance for a ray to then keep bouncing is thus $\rho(1-f)$, and its complement is $1-\rho(1-f)$. In equilibrium, the sphere loses photons at the same rate as it receives them, which means the input power, times the chance to stay in the sphere, $P_{0} \rho(1-f)$, must equal the power in the sphere times the chance for photons to disappear, $P(1-\rho(1-f))$. This leads to the flux enhancement factor 


$$
\frac{P}{P_{0}}=\frac{\rho(1-f)}{1-\rho(1-f)}=M(1-f),
$$

,

where the sphere multiplier $M$ is defined as

$$
M=\frac{\rho}{1-\rho(1-f)}
$$

The sphere multiplier is the most important parameter characterising an integrating spheres performance as it quantifies the gain in photon flux in comparison to the normal input power $P_{0}$ for a sphere of a given radius, reflectivity and port fraction. $M$ is strongly dependent on the reflectivity of the sphere surface and as such commercially available spheres tend to me made of diffuse materials that are highly reflective in the wavelength range of interest. The most common material available is Spectralon, manufactured by Labsphere, that exhibits an average reflectivity of $99 \%$ in the across the visible range.

\subsection{Absorption Spectroscopy in an Integrat- ing Sphere}

\subsubsection{Background}

A natural, but rare use of integrating spheres is to perform UV-Vis spectroscopy on liquid samples that either have a spatial dependence of their optical properties or are too turbid to be measured in a standard UV-Vis setup. Furthermore, the fact that the interaction path length of the light with the sample is increased provides another advantage towards measuring low concentrations of absorbing species. Such a setup has been applied in the past [117] numerous times but is mostly limited to measuring the absorption of seawater and microalgae [118-121] where scattering by suspended particles and the low concentrations of absorbing species can limit the use of standard UV-Vis absoprtion. It has been well established that integrating spheres used in such a fashion are unaffected by scattering [122], yet their limited use is mostly 
down to the complicated nature of the light propagation within the sphere when an absorbing, and/or scattering sample is present. Monte-Carlo methods have been developed $[115,122]$ to understand better the light propagation yet there have been very few reports of simple, quantitative procedures to extract the true absorbance of the sample [123]. In the following sections, the working principle of the CMCIS developed in the context of dyes molecules in colloidal nanoparticles is discussed and the method, used in Chapter 6, for extracting the true dye absorbance as can be compared with standard UV-Vis is introduced.

\subsubsection{Experimental Setup - Center Mounted Sample}

The optical schematic of the setup developed in this thesis is shown in Figure 5.4. The setup consists of modified Ocean Optics ISP-80 integrating sphere into which a cylindrical glass tube $(67.5 \mathrm{~mm}$ long, $7 \mathrm{~mm}$ external diameter, wall thickness $1 \mathrm{~mm}$ ) carefully inserted into the sphere through a port in the north pole. White light illumination is provided by a standard $100 \mathrm{~W}$ halogen lamp (See Fig. S2 for the lamp spectrum) and delivered to the sphere's equatorial port via a ThorLabs $1000 \mu \mathrm{m} 0.22 \mathrm{NA}$ optical fiber. Collection is made via a similar optical fiber $(910 \mu \mathrm{m}, 0.22 \mathrm{NA})$ inserted into a custom-drilled detection port in the bottom of the sphere, and coupled to an Ocean Optics USB-2000+ spectrometer with $100 \mu \mathrm{m}$ entrance slits and a detection range of $200 \mathrm{~nm}$ to $850 \mathrm{~nm}$. Figure 5.4 also for illustration purposes depicts the path of a number of rays as they reflect (diffusely as described earlier) throughout the sphere.

\subsubsection{Sample Holder Design}

The final experimental design is depicted in Figure 5.5. The sphere itself was used as is from the manufacturer (apart from the detection port drilled in the bottom) whereas the sample-holder-lid was designed and custom built specifically to allow a cylindrical glass vial to be inserted into the center of the sphere. The design of the sample holder ensures the vial is placed at identical positions because of the flange mount coupling with the inner surface of the lid. This design was crucial to the sensitivity obtained for the results present 


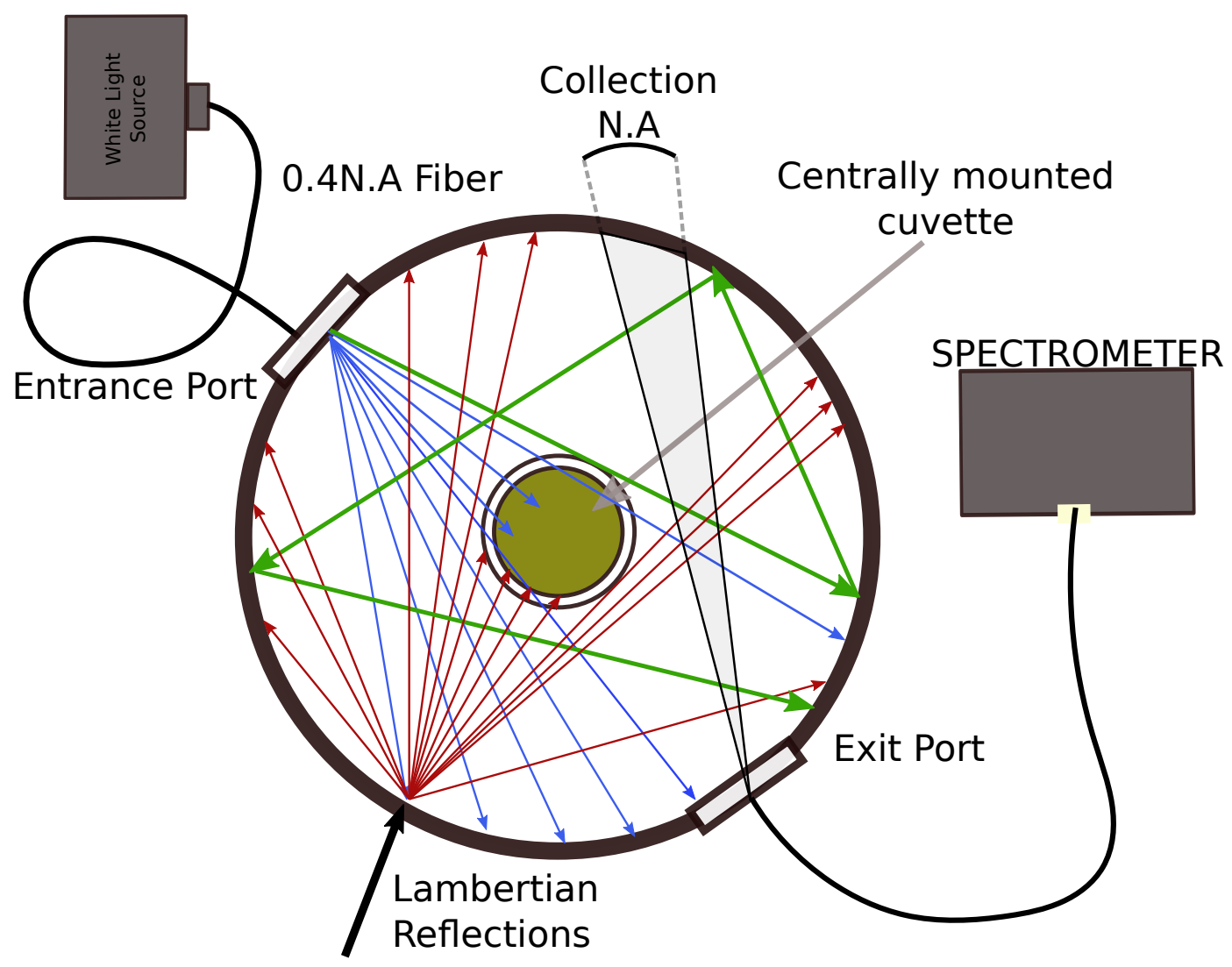

Figure 5.4: Schematic of the centrally mounted cuvette integrating sphere (CMCIS) setup used to measure the absorbance of dyes on nanoparticles. Light rays can travel a number of different paths through the sample and a few are depicted here. 
in Chapter 6. The sample holder body and lid were manufactured from teflon to ensure they matched the Spectralon coating of the sphere surface as closely as possible to minimise effects on the $\mathrm{M}$-factor calculations.

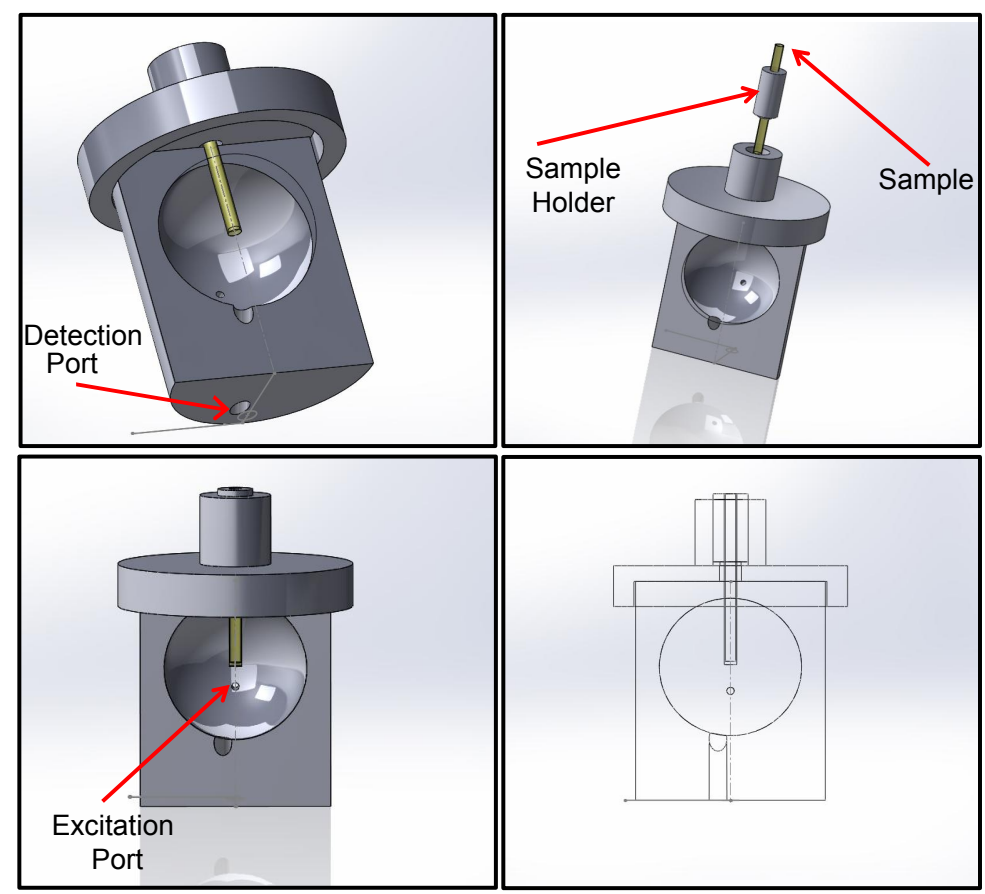

Figure 5.5: Cut-out drawing of the final sphere assembly design with the custom built lid and sample holder.

\subsubsection{CMCIS Absorbance Corrections}

The equations presented in Section 5.1.2 for determining the sphere $M$ factor are applicable to light travelling through an empty sphere that contains no absorbing sample. The integrating sphere is employed in this thesis however in the CMCIS configuration, to perform optical absorption spectroscopy, where a cuvette of absorbing (and possibly scattering) liquid is placed within the sphere cavity. The equations presented for an empty sphere must therefore be modified to understand how absorption spectroscopy can be performed in 
this configuration to allow direct comparison of spectra with those obtained through standard UV-Vis transmission.

The absorption spectrum of a solution is typically measured in a standard UV-Vis transmission setup, whereby white light is shone directly through a liquid sample of given length and the transmitted light is sent to a detector. By comparing the ratio of transmitted versus input light power, one can deduce the (wavelength dependent) absorbance of the sample, $A$, that is determined by the Beer-Lambert Law [55]:

$$
A=-\log _{10} \frac{P_{\text {trans }}}{P_{\text {in }}}=c_{m} \bar{\epsilon} L
$$

where the transmittance, $\mathrm{T}=\frac{P_{\text {trans }}}{P_{\text {in }}}, P_{i n}[\mathrm{~W}]$ is the incident power, $P_{\text {trans }}$ $[\mathrm{W}]$ is the transmitted power, $c_{m}[\mathrm{M}]$ is the concentration, $\bar{\epsilon}\left[\mathrm{cm}^{-1} \mathrm{M}^{-1}\right]$ is the molar extinction coefficient and $\mathrm{L}[\mathrm{cm}]$ is the path length of the sample, which is normally $1 \mathrm{~cm}$ in UV-Vis experiments. Absorbance is then dependent on sample path length so for comparison between instruments, the absolute absorbance in $\mathrm{cm}^{-1}$ is introduced, i.e. the absorbance that would be measured in a cell of path length of $1 \mathrm{~cm}$. Thus two samples with different path lengths can be normalised to path length to yield an absolute absorbance that is directly comparable ${ }^{1}$.

In the case of a sample placed within an integrating sphere, the path length is no longer trivially defined due to the increased probability for light rays to interact with the sample (through the sphere $M$ factor). As such, to obtain absolute absorbances $\left[\mathrm{cm}^{-1}\right]$ (as would be measured in a standard UV-Vis setup) from absorbance measurements the Beer-Lambert Law must be modified.

The sphere enhancement factor, $M$, can be approximately translated into an increased effective path length of the incident light with the sample compared with the path length for a standard transmission configuration without a sphere. This can alternatively be viewed as the average number of reflections a single photon will undergo within the sphere before either leaving through

\footnotetext{
${ }^{1}$ Note that the absolute absorbance has similar units to optical density but optical density is avoided because it generally refers to scattering as well as absorbance where as here the discussion is strictly limited to absorbance.
} 
a port or being absorbed by the sphere walls. The average chord length, or distance travelled by a ray in a sphere is given by [124]:

$$
L_{\mathrm{Av}}=\frac{2}{3} D,
$$

where $D$ is the diameter of the sphere. So $L_{\mathrm{Av}}$ constitutes the distance travelled by a ray in one bounce within the sphere. The sphere multiplier, $M$ then determines how many of these bounces occur within a sphere of a given $\rho$ and $f$. Thus, the total path length travelled by a ray is given by

$$
L_{\mathrm{Tot}}=M L_{\mathrm{Av}} .
$$

The above equations apply to a sphere that is filled entirely by the sample. For a setup such as the CMCIS, the effective path length is reduced approximately by the fraction of the entire sphere volume, $V_{\text {Sphere }}$ that the sample vial occupies. In the setup used here, the sample consists of a cylindrical vial of volume $V_{\text {Sample }}$. The effective path length in the sphere is then given by

$$
L_{\text {sphere }}=L_{\text {Tot }} \frac{V_{\text {Sample }}}{V_{\text {Sphere }}}
$$

where $V_{\text {Sphere }}$ is the total volume of the sphere. It follows that the maximum path length enhancement achievable would be for a sphere entirely filled with the sample to be measured but this is difficult to achieve in practice.

The best strategy to normalise the measured absorbance and obtain an absolute absorbance in $\mathrm{cm}^{-1}$ is to measure the effective path-length by a direct comparison with standard UV-Vis measurements, for a dye of known concentration.

In the absence of scattering, the two spectra should therefore coincide up to a multiplication factor, the ratio of effective path-length $L_{\text {Sphere }}(\lambda)$ (Eq. 5.6 to UV-Vis path length (which is the standard $1 \mathrm{~cm}$ in this case). Here $L_{\text {Sphere }}$ explicitly depends on wavelength but as shown this variation is negligible in the regions where the dyes of interest absorb. Fig. 5.6(a) presents the results 

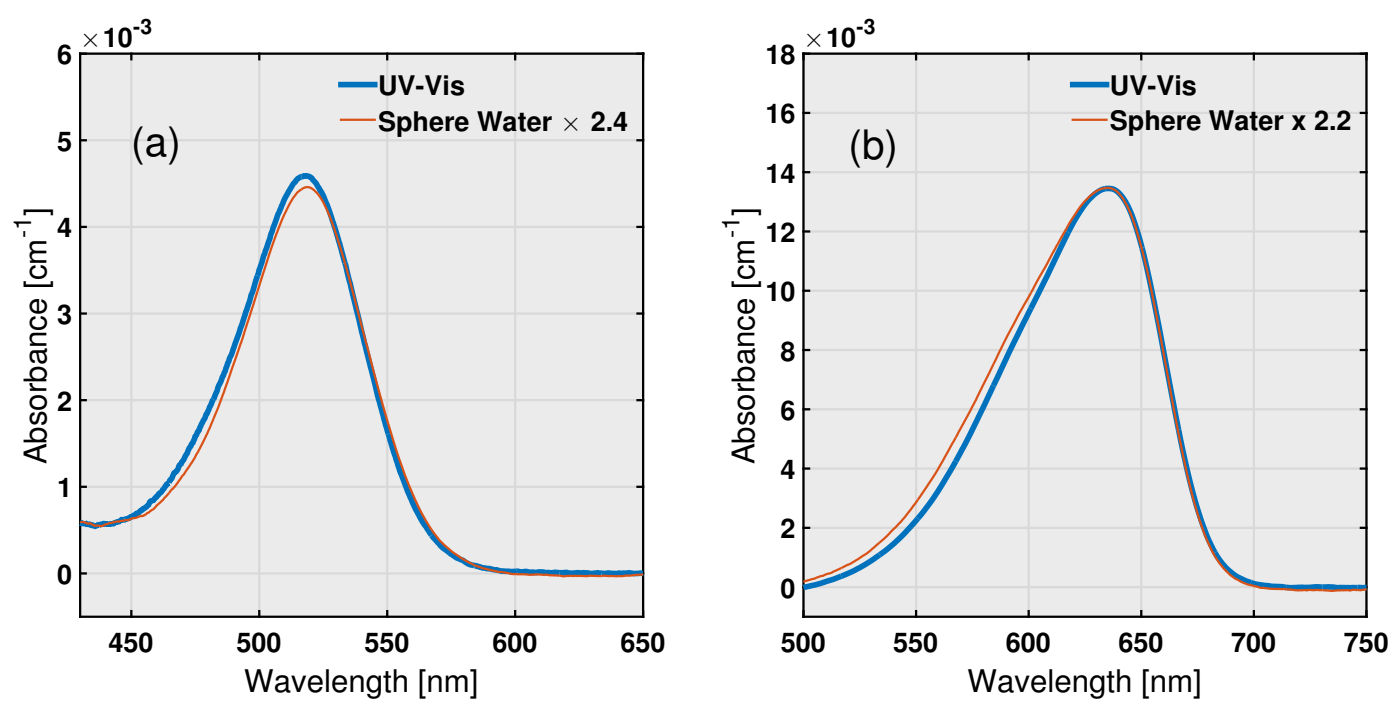

Figure 5.6: (a) Acquired absorption spectra of the dye Eosin B at $100 \mathrm{nM}$ against water measured in a standard UV-Vis setup with a $1 \mathrm{~cm}$ cuvette (blue) and inside the integrating sphere (red). (b) Similar results for Nile Blue which has a peak absorbance at $\sim 635 \mathrm{~nm}$ in water. From those measurements, the effective path length inferred is approximately constant at $L_{\text {Sphere }}=2.4 \mathrm{~cm}$ in the range $450-700 \mathrm{~nm}$.

of this experiment for the dye Eosin B measured in water in UV-Vis and integrating sphere configurations. The measured increase in path length due to the integrating sphere, $L_{\text {Sphere, }}$ was found to be approximately constant at $2.4 \mathrm{~cm}$ in the spectral range where Eosin B absorbs $(450-560 \mathrm{~nm})$. A similar test was carried out for Nile Blue (Figure $5.6(\mathrm{~b})$ ), which gives $L_{\text {Sphere }} \approx 2.2-$ $2.6 \mathrm{~cm}$ in the spectral range $560-700 \mathrm{~nm}$.

For the setup developed, the sphere and sample parameters are summarised in Table 5.1, where $r_{s}$ is the radius of the sphere, $r_{1}^{p}$ is the radius of the entrace port, $r_{2}^{p}$ the radius of the detection port, $f$ the port fraction and $\rho$ the sphere reflectivity. The sphere reflectivity is chosen here as 0.99 to closely match that of the sphere surface material, Spectralon. Spectralon is specifically designed so that sub-surface scattering gives close to perfect diffuse reflectivity (>99\%) across the UV-VIS-NIR region. The reflectivity is almost flat across the region of interest (350 to $900 \mathrm{~nm}$ for the measurements performed here) allowing a single value for $\rho$ to be used in calculating the predicted path length enhancement. Given that $M$ is strongly dependent on $\rho$, it is crucial for the sphere surface to exhibit the highest reflectivity possible. As such, the true reflectivity of the sphere surface is difficult to obtain directly but can be 
inferred indirectly from knowing the experimental $L_{\text {Sphere }}$ as found in Figure 5.6 .

\begin{tabular}{ccccccc}
\hline$r_{s}[\mathrm{~mm}]$ & $r_{1}^{p}[\mathrm{~mm}]$ & $r_{2}^{p}[\mathrm{~mm}]$ & $f$ & $\rho$ & $M$ & $L_{\text {Theo }}[\mathrm{cm}]$ \\
\hline 40 & 2 & 3.9 & 0.003 & 0.99 & 99 & 4.9 \\
40 & 2 & 3.9 & 0.003 & 0.985 & 66 & 3.3 \\
40 & 2 & 3.9 & 0.003 & 0.98 & 49 & 2.4 \\
\hline
\end{tabular}

Table 5.1: Sphere dimensions and parameters used for calculating the effective path length, $L_{\text {Theo }}$. A sphere reflectivity of $98 \%$ is required to agree with the measured path length of $2.4 \mathrm{~cm}$

Using Eqs. 5.2 and 5.6 and assuming the $\rho$ has no wavelength dependence, the predicted sphere $M$ factors are shown in Table 5.1 for three values of $\rho$. For a cylindrical vial of height $67.5 \mathrm{~mm}$ and (inner) radius $2.5 \mathrm{~mm}$ placed within the sphere of given dimensions, a sphere reflectivity of $98 \%$ predicts a path length enhancement, $L_{\text {Theo }}$ of $2.4 \mathrm{~cm}$. This then constitutes an enhancement with respect to a standard $10 \mathrm{~mm}$ cuvette pathlength of 2.4. Since the calculated pathlength is strongly dependent on the exact sphere reflectivity, the constant $L_{\text {Sphere }}$ of $2.4 \mathrm{~cm}$ was chosen for simplicity to convert all sphere absorbance spectra into absolute absorbance in $\mathrm{cm}^{-1}$ and is in good agreement with theoretical predictions.

Given these calibrations, all sphere spectra were corrected for the effective path-length to obtain absolute absorbance values in $\mathrm{cm}^{-1}$ as follows:

$$
A(\lambda)=\frac{A_{\text {Sphere }}(\lambda)}{L_{\text {Sphere }}} \quad \text { with } \quad L_{\text {Sphere }}=2.4 \mathrm{~cm} .
$$

With the above calibration, the spectra obtained from the integrating sphere can now be correctly converted into a corresponding standard ab- 
sorbance as would be obtained in a UV-Vis transmission setup. If required, the absorbance $\left[\mathrm{cm}^{-1}\right]$ can then be converted to a molar extinction coefficient, $\bar{\epsilon}$ in $\mathrm{cm}^{-1} \mathrm{M}^{-1}$ by normalising to the sample concentration.

\subsubsection{Standard Absorbance Measurement Steps}

The steps in obtaining the raw absorbance spectrum of a solution as presented in the previous section are outlined as follows. Each sample requires a reference spectrum to be taken, which generally is the pure solvent in which the sample is diluted. For dyes this will be a water solution while in chapter 6 the reference solution is the bare colloids. Thus to perform the measurement, the transmitted intensity for the reference solution and then the sample solution are obtained under identical acquisition parameters. Absorbance spectra are obtained as would be using the standard Beer-Lambert law:

$$
A_{\text {Sphere }}(\lambda)=-\log _{10} \frac{I_{\text {Sample }}}{I_{\text {Reference }}}
$$

where $I_{\text {Sample }}$ and $I_{\text {Reference }}$ are, as the names suggest, the intensities of the sample and reference solutions, respectively.

Figure 5.7 shows the results of this approach with the transmitted lamp spectra for a reference solution of water and a sample solution of $100 \mathrm{nM}$ Eosin $\mathrm{B}$ in water. The lamp output for all spectra was set to $100 \mathrm{~W}$ and integration times of $1 \mathrm{~ms}$ were used. The spectra are plotted from 300-900 nm (a) and the resulting asborbance spectrum obtained through Equation 5.8 is plotted in (b). As can be seen from the raw lamp spectra, the lamp intensity drops off significantly below $\sim 400 \mathrm{~nm}$ due to the halogen lamps weak UV output. Nevertheless, with sufficient averaging, the lamp output provides enough intensity across the visible region to perform the measurements in Chapter 6. Note that by taking the ratio of intensities, absorbance spectra are automatically normalised with respect to the lamp spectrum; the lamp intensity is only an important factor for the signal-to-noise ratio, particularly in the blue-near-UV, or if it presents spectral drifts over time (this was checked to be of negligible effect in the set-up). The maximum counts were kept at 12000 to avoid any non-linearities in the spectrometer CCD. 

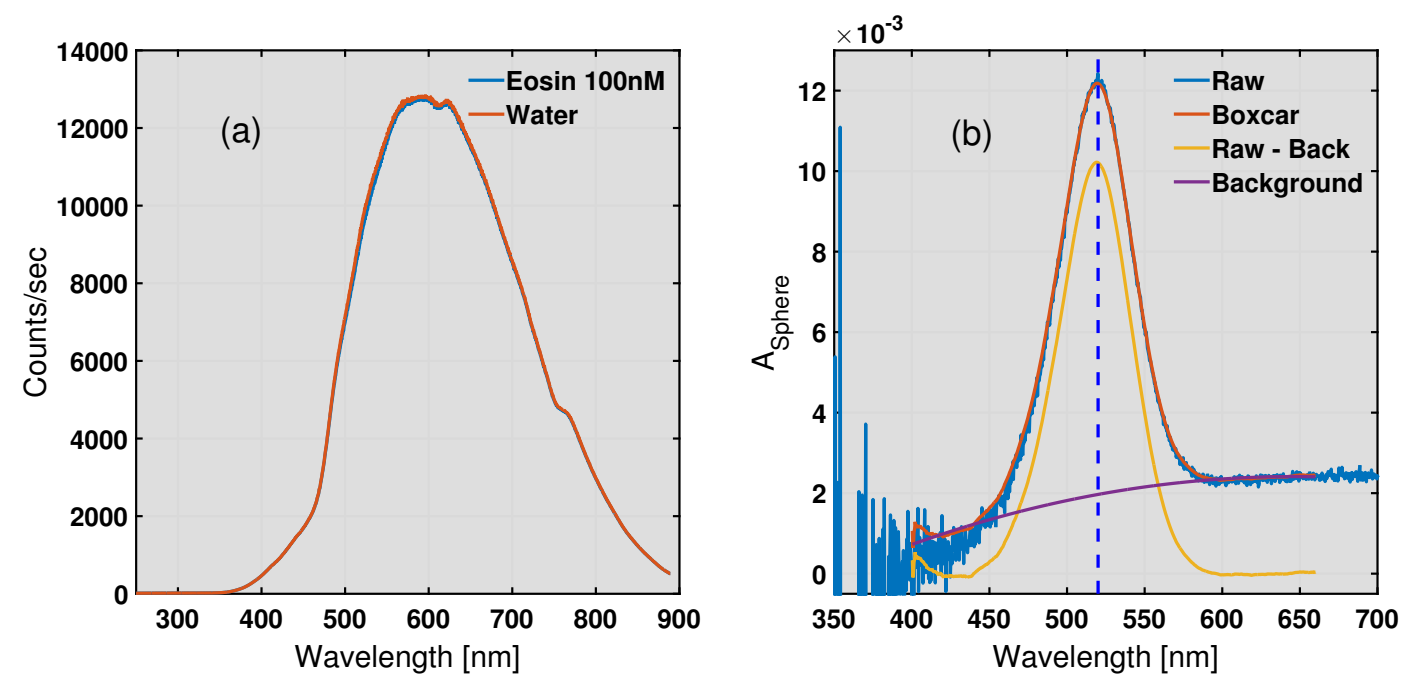

Figure 5.7: (a) The reference spectrum of the halogen lamp with a water solution (red) and $100 \mathrm{nM}$ Eosin B (blue) in water in the sphere. Integration time was $1 \mathrm{~ms}$ and the spectrum is averaged over 10000 spectra. (b) The post processing steps involved in obtaining the absoprtion spectrum of the dye; the raw data (blue) obtained from the Beer-Lambert Law is then smoothed (red) and a second order background (purple) is subtracted to yield the final cleaned spectrum (orange).

Raw data is post-processed by applying a 31-pixel moving average filter to remove noise yet not affect spectral shape (Figure 5.7 (b)). Slow drifts in the lamp spectrum slightly inaccurate referencing or small changes in sample geometry can cause variations in measured intensities resulting in spectrally smooth backgrounds in the absorbance measurements. To account for these instrumental limitations, a standard baseline correction is performed by subtracting a second-order polynomial from the raw absorbance spectrum in the region of interest as shown in Figure 5.7 (b).

\subsubsection{Differential Absorbance in the Integrating Sphere}

In Chapter 6, the absorbance of dye molecules on the surface of nanoparticles is measured in the integrating sphere. This method is denoted as differential absorption where the dye spectrum is obtained by using the NP-only solution as a reference, instead of water. This is equivalent to taking the difference between the absorbance spectrum of the NP-dye and NP solutions, and yields 

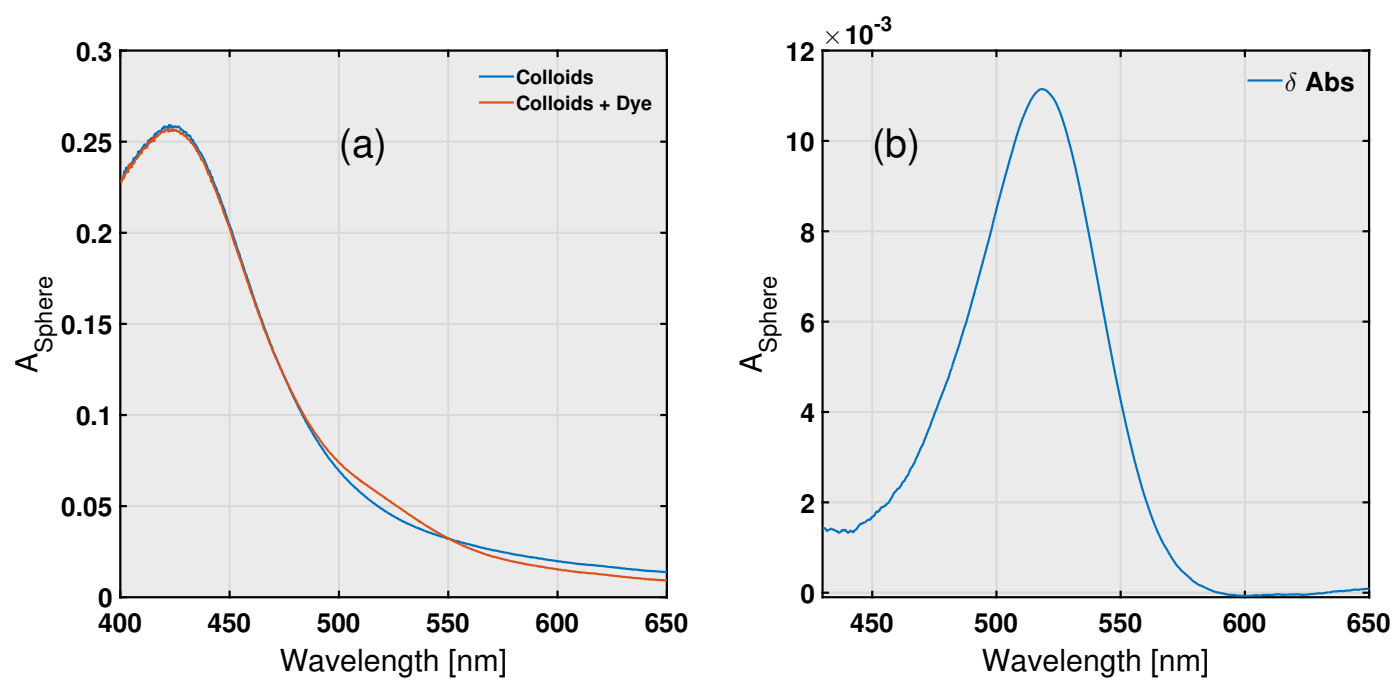

Figure 5.8: (a) The absorbance spectra of the Ag colloids ( $8 \mathrm{pM})$ and mixture of Ag colloids ( $8 \mathrm{pM})$ and the dye Eosin B (100 nM) measured in the CMCIS. (b) Differential absorption spectrum of Eosin B obtained from applying Equation 2.9 to the spectra in (a).

the differential sphere absorbance spectrum for the dye given by:

$$
\delta A_{\text {Sphere }}(\lambda)=-\log _{10} \frac{I_{\mathrm{NP}+\text { Dye }}}{I_{\mathrm{NP}}}=A_{\mathrm{Sphere}}^{\mathrm{NP}+\text { dye }}(\lambda)-A_{\mathrm{Sphere}}^{\mathrm{NP}}(\lambda) .
$$

For illustration, Figure 5.8 (a) shows the uncorrected absorbance spectra (water reference) for a solution of Ag colloids at a concentration of $8 \mathrm{pM}$ and the same solution but with $100 \mathrm{nM}$ of Eosin B added. Equation 5.9 can then be used to obtain the differential absorbance spectrum as shown in Figure 5.8 (b). The final step to convert this absorbance into an absolute absorbance (in $\mathrm{cm}^{-1}$ ) is done by dividing the spectrum in (b) by the sphere path length, $L_{\text {Sphere }}$.

\subsubsection{Sphere Linearity Response}

The equations presented above are in principle only valid up to a certain value of absorbance within the sphere, as found by the non-linear response with increasing absorption [122]. For a sphere of given radius and port fraction, there will be a point at which the sample absorbance begins to play a role in 
the measured absorbance; as such, within the sphere the measured absorbance quickly becomes non-linear with sample concentration, putting a limit on the dynamic range of the integrating sphere setup being used. While the reasons for these deviations are complicated and difficult to account for, a simple description is that for highly absorbing samples, the path length enhancement of light within the sphere is no longer simply defined by Equation 5.2. These effects can be mitigated as shown below by working within the linear response range of the sphere, where the simple equations are still valid.

The response of CMCIS setup to sample absorbance was tested by measuring increasing concentrations of the dye Eosin B within the CMCIS and in standard UV-Vis transmission configurations. By comparing the measured absorbances in the CMCIS to the real absorbance in transmission, the validity of the signals measured in the CMCIS can be tested.
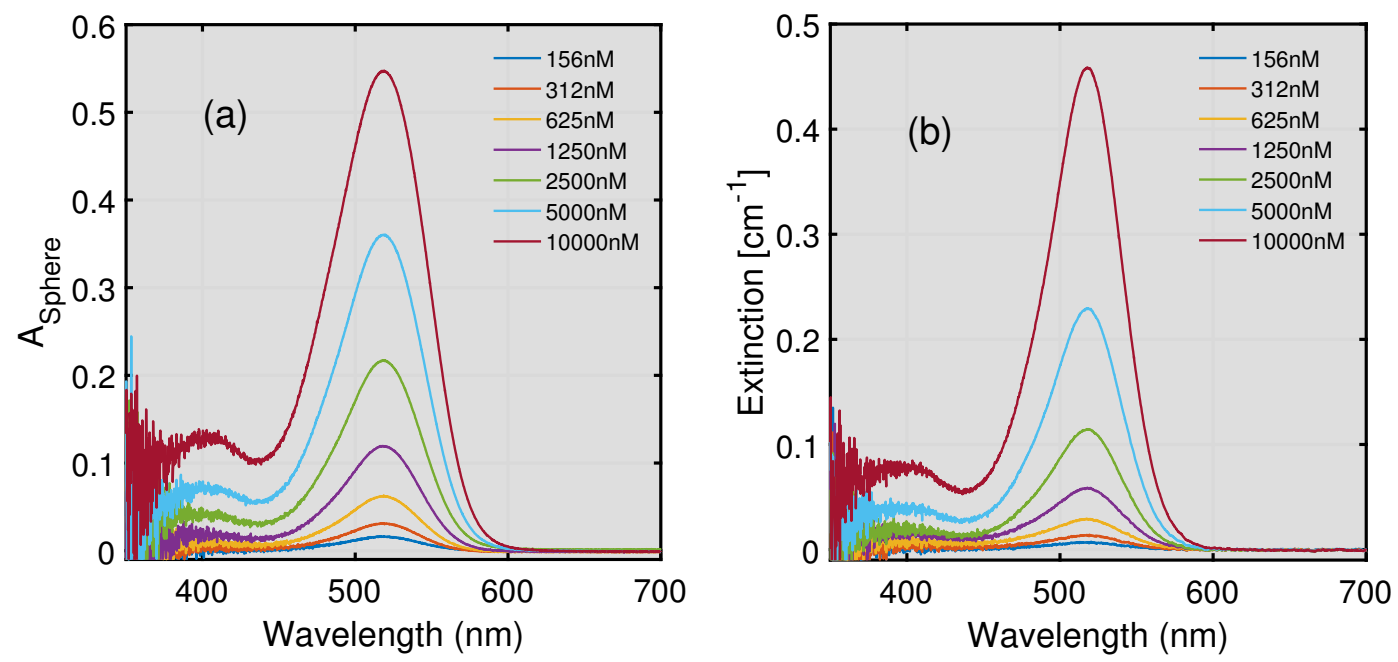

Figure 5.9: (a) Absorption spectra, A Aphere as measured in the CMCIS of Eosin B at increasing concentrations. (b) Extinction (transmission) spectra of the same solutions of Eosin B.

The results of these measurements are shown below. In Figure 5.9 the raw absorption spectra of Eosin B at concentrations increasing from $156 \mathrm{nM}$ up to $10 \mu \mathrm{M}$ measured in (a) the CMCIS and (b) transmission configurations are shown. The CMCIS data is plotted as measured, i.e. without the $L_{\text {Sphere }}$ factor removed while the transmission data is plotted as extinction ${ }^{2}$ in $\left[\mathrm{cm}^{-1}\right]$. Figure

\footnotetext{
${ }^{2}$ Note here that transmission data are plotted and referred to as extinction to distunguish from those measured in the sphere but in reality are eqiuvalent to absolute absorbance.
} 
5.10 highlights the departure from linearity in the CMCIS, where the maximum absorbances (taken at $517 \mathrm{~nm}$ ) of the extinction (red dots), absorbance in the sphere (blue dots) and the extinction spectra scaled by $L_{\text {Sphere }}$ (yellow triangles) are plotted. In (a), the extinction maxima vs concentration show almost perfect linearity as expected, while the CMCIS absorbance clearly begin to saturate beyond $600 \mathrm{nM}$, or an absorbance of about 0.06 . The scaled extinction spectra show a distinct disagreement with the measured absorbance in the sphere, indicating that $L_{\text {Sphere }}$ is no longer independent on the absorbance of the sample. In (b), the same plots are shown in the 0 to $600 \mathrm{nM}$ region, where the CMCIS absorbance is linear, and applying the $L_{\text {Sphere }}=2.4$ factor is valid within less than $10 \%$ error.

From these measurements, it is clear that working at dye concentrations typically less than 500-600 nM (depending on the particular extinction coefficient of the dye in use) ensures the linear scaling method as described is a valid approach for converting the measured CMCIS absorbance to the true absorbance as measured in transmission. In the proceeding Chapter, all dye concentrations are much below this limit with the maximum concentration used being $25 \mathrm{nM}$. Nevertheless the above calibration is crucial towards ensuring accurate values of absorbance are obtained.
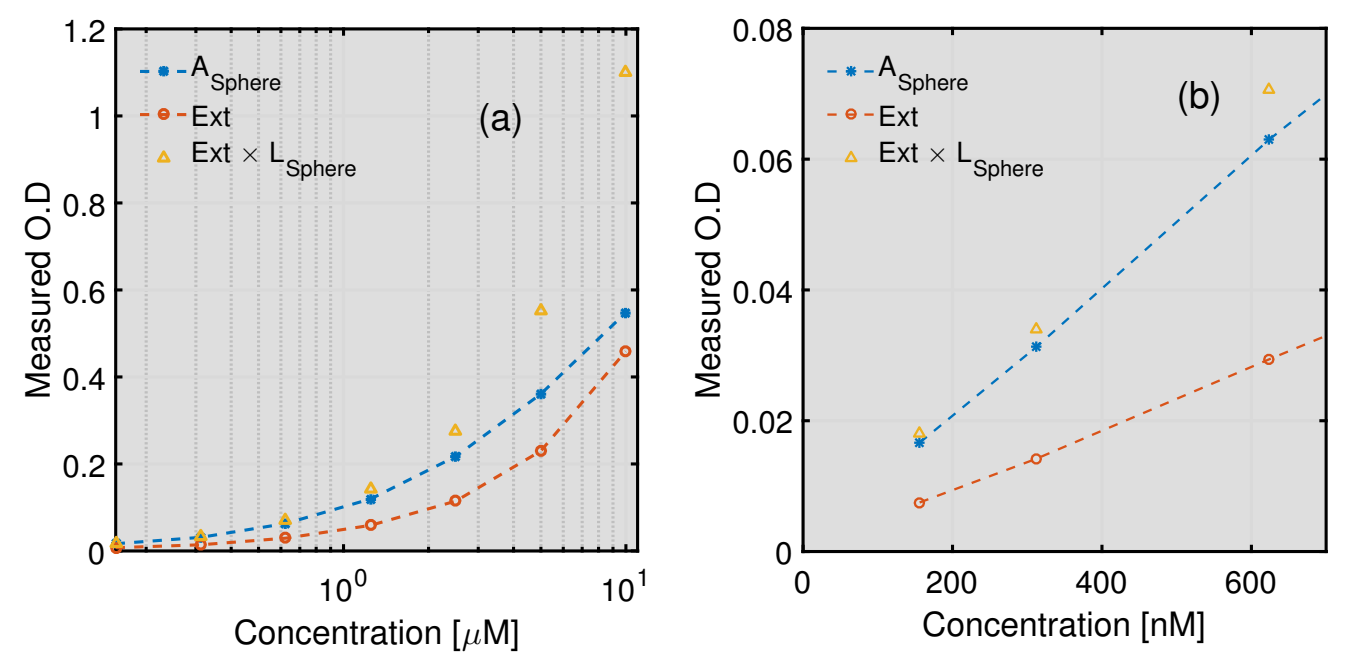

Figure 5.10: (a) Optical density of Eosin B measured at $517 \mathrm{~nm}$ vs concentration in the CMCIS (blue dots), standard extinction (red circles) and the extinction scaled by the sphere enhancement, $\mathrm{L}_{\text {Sphere }}=2.4$, versus (log) concentration. Note that above $600 \mathrm{nM}$ the factor of 2.4 no longer applies in the sphere. (b) Zoomed region showing the linear response of the CMCIS at low absorbances and deviation beyond $600 \mathrm{nM}$ (above 0.06 absorbance). 


\section{Chapter 6}

\section{Modified Absorbance of Molecules Adsorbed to Metallic Nanoparticles}

This chapter presents efforts carried out to experimentally measure the absoprtion spectrum of dye molecules adsorbed to metallic nanoparticles. A novel experimental setup, consisting of an integrating sphere with a centre mounted cuvette (CMCIS), described in Chapter 5, was developed allowing the surface absorbance of molecules on colloidal nanospheres at concentrations in the sub-monolayer regime to be measured. The results are the first direct measurements of such absorbances, where the low concentration of dyes ensures that molecule-molecule interactions do not play a role in the observed spectra ${ }^{1}$. Measuring such low absorbance in a medium with a large scattering background poses a significant experimental challenge and the CMCIS setup developed is crucial to achieving the sensitivity required to measure these signals accurately.

The observation of distinct shifts and broadening of dye absorption spectra on silver colloids compared to the free dye absorption provides direct evidence that the electronic states of the molecules are modified when adsorbed to

\footnotetext{
${ }^{1}$ It is important to note that near-field mediated interactions between molecules, such as enhanced Forster Resonance Energy Transfer (FRET), are not considered in the treatment of the dye-nanoparticle system [125-127].
} 
metallic surfaces. These shifts are fundamental to understanding the mechanisms at play in any surface enhanced spectroscopy where resonant absorption of light occurs and contribute significantly to the long standing Chemical Enhancement debate in SERS. The method developed also allows investigation of the magnitude of the absorption enhancement for dye molecules on nanostructures which has to now gone unmeasured directly. It is shown that the measured enhancements are significantly less than expected from Mie-theory predictions and can vary depending on the nature of the dye molecule used.

This chapter begins with a background discussion of the motivation behind measuring the absorbance of molecules on metallic nanoparticles. The challenges faced in measuring these spectra are discussed in relation to retrieving the dye absorbance signal among the overwhelming nanoparticle extinction. Mie theory shell modelling is then employed to reveal why low dye coverages are required to extract the true surface absorbance of dye molecules, adding further to the difficulty of the experiment. Surface absorbance measurements are then presented for four commonly used dye molecules adsorbed to $60 \mathrm{~nm}$ silver nanospheres; Nile Blue, Rhodamine 6G, Rhodamine 700 and Crystal Violet at sub-monolayer concentrations, approaching those used in single molecule SERS experiments. The observations and implications of distinct shifts and broadening of molecular absorbances for surface enhanced spectroscopies are then discussed, along with the magnitude of the absorption enhancement, and how these enhancements can be correlated with those measured for Raman signals.

Most of the work presented in this chapter was published in Nature Photonics [128]: doi:10.1038/nphoton.2015.205 


\subsection{Motivation}

At the heart of most surface enhanced spectrosocpic techniques, whereby an emitter (dye molecules, quantum dots) is placed at the surface of a nanostructure, is the idea that the electronic absorbance of the emitter is enhanced by the local electric field at the nanostructure surface. While not explicitly the case for non-resonant excitation, any surface enhanced technique where one works with dye molecules that are electronically resonant with the incoming laser, then electronic absorption plays a key role in the observed enhancements. All of the molecules used so far in this thesis fall in this category, where resonant excitation allows one to benefit from the much larger $\left(10^{4}\right)$ resonant Raman cross sections [28]. In cases such as SERS at (molecular) resonance, it is natural to assume that if the (effective) Resonant Raman cross section of the molecule is larger when situated at the metal surface, there must be a corresponding enhanced electronic absorption simply due to the larger electric fields present at the metal surface.

Enhanced absorbance can be understood in the simplest approximation (following the treatment in Section 4.6.2 of Ref [16] by considering a molecule with linear optical polarizability, $\hat{\alpha}_{L}(\omega)$, excited by an incident field $\mathbf{E}$ of frequency $\omega_{L}$. Here the polarizability is explicitly tensorial in nature but for simplicity it is assumed the tensor is uni-axial along the axis of the molecule, $\mathbf{e}_{m}$ (which is valid for most dye molecules whose dipole moment in oriented along the backbone of the molecule). The complex scalar polarisabilty, $\alpha_{L}$ is then introduced through $\hat{\alpha_{L}}=\alpha_{L} \mathbf{e}_{m} \otimes \mathbf{e}_{m}$. The dipole induced by the field ${ }^{2}$ (oriented along the molecular axis associated with $\alpha$ ) is then

$$
\mathbf{p}_{L}=\alpha_{L} \cdot \mathbf{E}
$$

Thus for plane wave exctiation and randomly oriented molecule, the power absorbed by this dipole is then given by [63]

\footnotetext{
${ }^{2}$ This approximation is not strictly valid for a molecule fixed to a surface and neglects any anisotropy in the polarizability that may be imposed by a fixed adsorption orientation on the metal surface, akin to Surface Selection Rules in SERS [10]. These effects are discussed further in Section 6.4.1
} 


$$
P_{A b s}=\frac{L_{M}^{1 / 2} \omega_{L}}{2}\left|E_{\operatorname{Inc}}\right|^{2} \frac{\operatorname{Im}\left(\alpha_{L}\right)}{3}
$$

where $L_{M}$ is the local field correction factor for the medium (here water), $E_{\text {Inc }}$ is the amplitude of the incident field and the factor of $1 / 3$ accounts for orientation averaging of the molecule. More relevant experimentally than the power absorbed by the dipole is the absorption cross section, i.e. the ratio of incident power to absorbed power. With the power density of the incident field given by

$$
S_{\mathrm{Inc}}=\frac{n_{M} \epsilon_{0} c\left|E_{\mathrm{Inc}}\right|^{2}}{2}
$$

where $n_{M}$ is the refractive index of the embedding medium, the absorption cross section is then

$$
\sigma_{\mathrm{Abs}}=\frac{P_{\mathrm{Abs}}}{S_{\mathrm{Inc}}}=\frac{\left(L_{M}^{1 / 2}\right) \omega_{L}}{n_{M} \epsilon_{0} c} \frac{\left.\operatorname{Im}_{(} \alpha_{L}\right)}{3} .
$$

Equation 6.4 underpins UV-Vis spectroscopy to the physical polarizabilty of the molecule, linking the (measured) absorption cross section to the imaginary part of $\alpha_{L}$ (See Appendix A for more details). For a molecule placed on the surface (not explicitly a metal but assumed here to be a nanoparticle), the absorption cross section on the surface, $\sigma_{A b s}^{S}$ is

$$
\sigma_{A b s}^{S}=M_{\mathrm{Loc}}\left(\omega_{L}\right) \sigma_{A b s} .
$$

The absorption enhancement factor is then simply defined in the same way as the Raman enhancement factor except that unlike Raman, there is no emission step so the absorption only benefits from the square of the local field, $M_{\text {Loc }}\left(\omega_{L}\right)$ as defined in Chapter 2. Thus comparing the surface absorption cross section to that measured in water yields the absorption enhancement factor:

$$
M_{\mathrm{Abs}}=\frac{\sigma_{A b s}^{S}}{\sigma_{A b s}}
$$


The closely related technique surface enhanced fluorescence (SEF) more crucially relies on the absorption of light by an electronic state of the emitter $[129,130]$, whereby in the right conditions, the enhanced local field increases the excitation rate of the molecule, leading to enhanced emission rates i.e. fluorescence, depending on the distance between the molecule (or quantum dot) and the metal surface. Because the magnitude of the fluorescence enhancement is more strongly dependent on the modification of the emitter decay rates when in proximity to the metal [16], enhanced absorbance does not imply an enhancement in fluorescence but it is still of interest to understanding enhanced fluorescence. Furthermore, given the direct link between absorption and fluorescence spectra, any modification of the absorption spectrum (in terms of spectral shape and shifts in resonance as well as intensity) will have implications for the fluorescence spectrum. It would thus be a major step forward for the SEF community to have direct access to the absorption spectra of emitters placed close to metallic nanostructures.

Finally in single-molecule SERS, the majority of studies are performed with resonant dye molecules, with only very few observations of non-resonant single molecule detection $[131,132]$, further solidifying the idea that working at resonance with the analyte yields significant advantages.

Given these considerations, it is surprising that there has been no direct evidence in the literature of the isolated surface absorbance spectra of dye molecules on metallic nanostructures. The dye-sensitized-solar-cell (DSSC) field has for a number of years looked at using plasmonic nanoparticles to enhance the absorption of the dye sensitizer [133], and in many cases there have been indications that the absorption spectrum of the dye molecule can be modified and/or enhanced by interaction with the (metallic) nanoparticle [134-138]. Most of these studies however do not attempt to isolate the true absorption spectra of the dye molecules and work with large surface dye concentrations, on flat substrates or in media that differ from the bare dye in solution, all of which work to hide the optical properties of the bare dye.

There are also numerous mentions of plasmon-molecule interactions in the sub-field of plasmonics now known as plexcitonics(discussed in Section 6.2.3). Here hybrid plasmon-exciton materials are created that exploit coupling between dye-molecule resonances and plasmon resonances to form exotic optical 
states that do not correspond to the sum of the individual dye and plasmon resonances. In the majority of these studies, the intrinsic dye properties on the surface cannot be extracted due to either dye-dye interactions from the large concentrations of dyes used or interactions with the plasmonic resonance, both of which complicate the observed dye spectrum. With this in mind, it was decided to investigate the possibility of measuring the absorbance profiles of commonly used dye molecules on the surface of the most widely used SERS substrate, spherical silver colloids, at concentrations where dye-dye interactions are negligible and where the detuning of the dye resonance from the plasmon resonance is large.

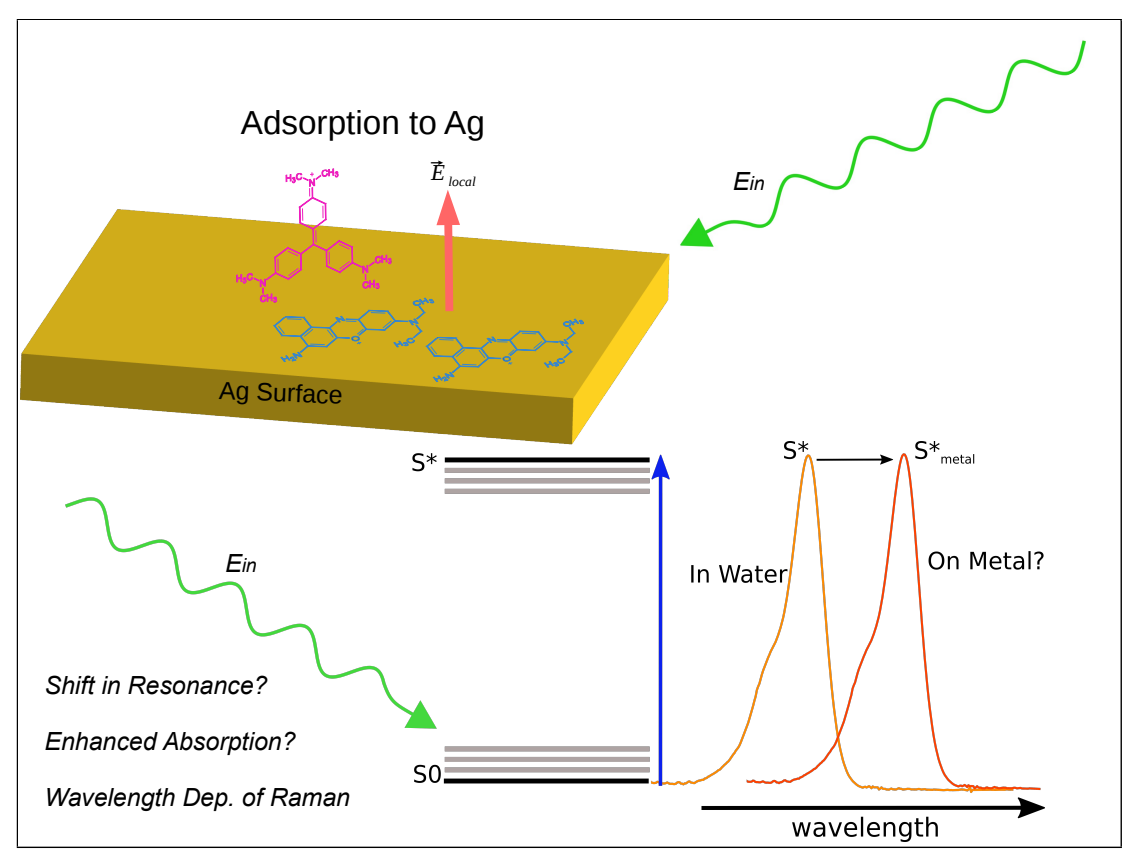

Figure 6.1: Qualitative depiction of the metal-induced shift of the electronic resonance of molecules adsorbed to a metal nanostructure. Upon adsorption to the metal surface, the molecules electronic states are modified through chemical interaction with the metal, thus causing a shift in the UV-Vis absorption spectrum. Access to this "surface" absorbance is experimentally challenging because of the low concentration of adsorbed dye and its relatively weak absorption compared to that of the metal structure.

The three main reasons for undertaking this study are discussed in more detail below. 


\subsubsection{Chemical Enhancement in SERS}

As alluded to in Section 2, probably the longest standing debate in the SERS field is the role, if any, of chemical effects contributing to the enhanced Raman signal observed from molecules on metallic surfaces. There are many excellent review articles $[19,37]$ on this topic and it would be fruitless to discuss every aspect of the debate but it is worthwhile to discuss the most pertinent aspects of the problem.

Since the discovery of the SERS effect, the debate over the origin of the huge enhancements observed has permeated the field, creating essentially two schools of thought; the first, and now widely accepted theory is that the enhanced Raman signal is primarily due to electromagnetic effects arising from surface plasmons excited within the metal nanostructure. First proposed by Moskovits in his seminal paper in 1978 [9], the EM treatment of SERS is now accepted to account for the majority of the enhancement [37] and has been confirmed many times by many different approaches [38]. In particular, the average SERS enhancements observed from nanostructures have in many cases been found to be in excellent agreement with electromagnetic predictions [70,131].

The second school of thought refers to a "Chemical Enhancement" whereby the chemical or electronic structure of the probe molecule is modified upon adsorption to the metal surface through a chemical bond between the molecule and metal surface. This bonding can affect the molecules Raman spectrum in 3 ways; (1) a change in the molecule's ground state electronic configuration will effect both the frequencies and intensities of the vibrational modes, (2) any perturbation of the intra-molecular electronic transitions (ground to excited states) by the metal surface will modify the electronic polarizability of the molecule and thus effect the resonance Raman cross sections and (3) the possibility of new metal-molecule or molecule-metal charge-transfer states may be created if the Fermi level of the metal and the unoccupied orbitals of the molecule lie close enough to allow a transfer of charge distribution across the molecule-metal system. Put simply by McCreery [56]:

The adsorbate-substrate complex has a different electron distribution from the isolated components, resulting in a different (Raman) cross section. 
Often, studies will infer a chemical effect in a number of ways; (1) observed changes in relative intensities of Raman bands [139] that have different symmetries (totally vs non-totally symmetric modes) in SERS can indicate selective charge-transfer transitions, (2) the appearance of new peaks or shifts $[140,141]$ with respect to the bare molecule can indicate the formation of new bonds between the molecule and metal, (3) by placing a spacer layer between the metal and the molecule layer, chemical effects can be effectively "switched" off, (4) varying the halide group on the nanoparticle surface can modify the chemical bond with the molecule [41] which can possibly be detected in the SERS spectrum. These results are often very specific to the system being studied and often require advanced theoretical treatments to explain the observed Raman intensities [142].

The Chemical Enhancement was one of the original candidates for understanding the origin SERS effect [10] and many descriptions have been developed to describe the process [34-36,143,144]. Despite the seemingly hostile nature of the debate that is rooted in history, today the Chemical Enhancement is thought to contribute at most a factor of $10^{2}$ towards the overall Raman enhancement [42]. Nevertheless, direct measurement of this enhancement is still extremely difficult because decoupling the EM enhancement from the Chemical Enhancement is often not possible. Furthermore for resonant molecules, any interpretations of Chemical Enhancements require a knowledge of how each Raman mode is coupled to the electronic states of the molecule. In many studies resonance Raman cross sections are not known for the molecules and as such comparison with SERS spectra are not valid against powder or non-resonant spectra. This point will be addressed in the following section.

\subsubsection{Wavelength Dependence of SERS at Resonance}

A major obstacle with SERS that has for many years hindered accurate estimates of EFs is the difficulty in accessing the normal (in water) Raman cross sections of the dye molecules used. While working at resonance with the molecule in SERS significantly improves sensitivity due to larger Raman cross sections, resonance Raman measurements in solution (non-SERS) are notoriously problematic because of the often overwhelming fluorescence signal from 
the dye molecules [145]. This fluorescence generally swamps the underlying Raman spectra which is typically $10^{7}$ times larger than the (resonant) Raman signal $[28,79]$. The "standard" weapon to tackle the fluorescence problem is to use ultrafast spectrosocpic techniques that can observe Raman vibrations before fluorescence (lifetimes of 10s of nanoseconds) occurs [60]. These techniques tend to be quite elaborate and cumbersome so a full wavelength dependence of the resonance Raman cross sections of dyes is often unavailable. As such, for any SERS measurement where the wavelength of the laser is varied, reliable enhancement fators for resonant dyes cannnot be calculated without knowledge of the normal Raman cross section at that wavelength.

A demonstration of this issue is apparent from the literature, where as of 2008 the Raman cross section of Rhodamine $6 \mathrm{G}$ at $533 \mathrm{~nm}$ excitation had been reported once [60]. Despite the deadlock broken by Shim et. al, not all labs researchers have access to femtosecond laser setups and so direct access to Resonant Raman cross sections of dyes is still technically challenging. Recent work from our group to alleviate the fluorescence swamping has resulted in two novel techniques use polarization differences [80] and CCD-shifting [81] to access resonant Raman cross sections with standard continuous-wave (CW) lasers. This development has allowed access to, for example, the full (514 nm to $647 \mathrm{~nm}$ ) Raman Exctitation Profile (REP) of Nile Blue and the cross section of Rhodamine $6 \mathrm{G}$ at $514 \mathrm{~nm}$ (extremely close to resonance). Using CW excitation simplifies both the experiment and analysis of Raman peaks.

Such work has opened a new avenue towards exploring the effect adsorption to a metal nanostructure has on the REP of dye molecules. Figure 6.2 shows the REP of the $595 \mathrm{~cm}^{-1}$ and $1650 \mathrm{~cm}^{-1}$ mode of Nile Blue obtained from the CCD-shifting technique in Ref [81]. Interestingly, it was found that the strength of the Raman mode of energy $\bar{\nu}_{v}$ versus excitation energy, $\bar{\nu}_{L}$ can be computed from the lineshape of the absorption spectrum $\sigma_{a b s}(\bar{\nu})$ of the molecule through

$$
\mathrm{d} \sigma_{\Omega}^{R}\left(\bar{\nu}_{L}\right)=A_{v}\left(\bar{\nu}_{L}-\bar{\nu}_{v}\right)^{4} \mid \varphi\left(\bar{\nu}_{L}-\left.\varphi\left(\bar{\nu}_{L}-\bar{\nu}_{v}\right)\right|^{2},\right.
$$

where 

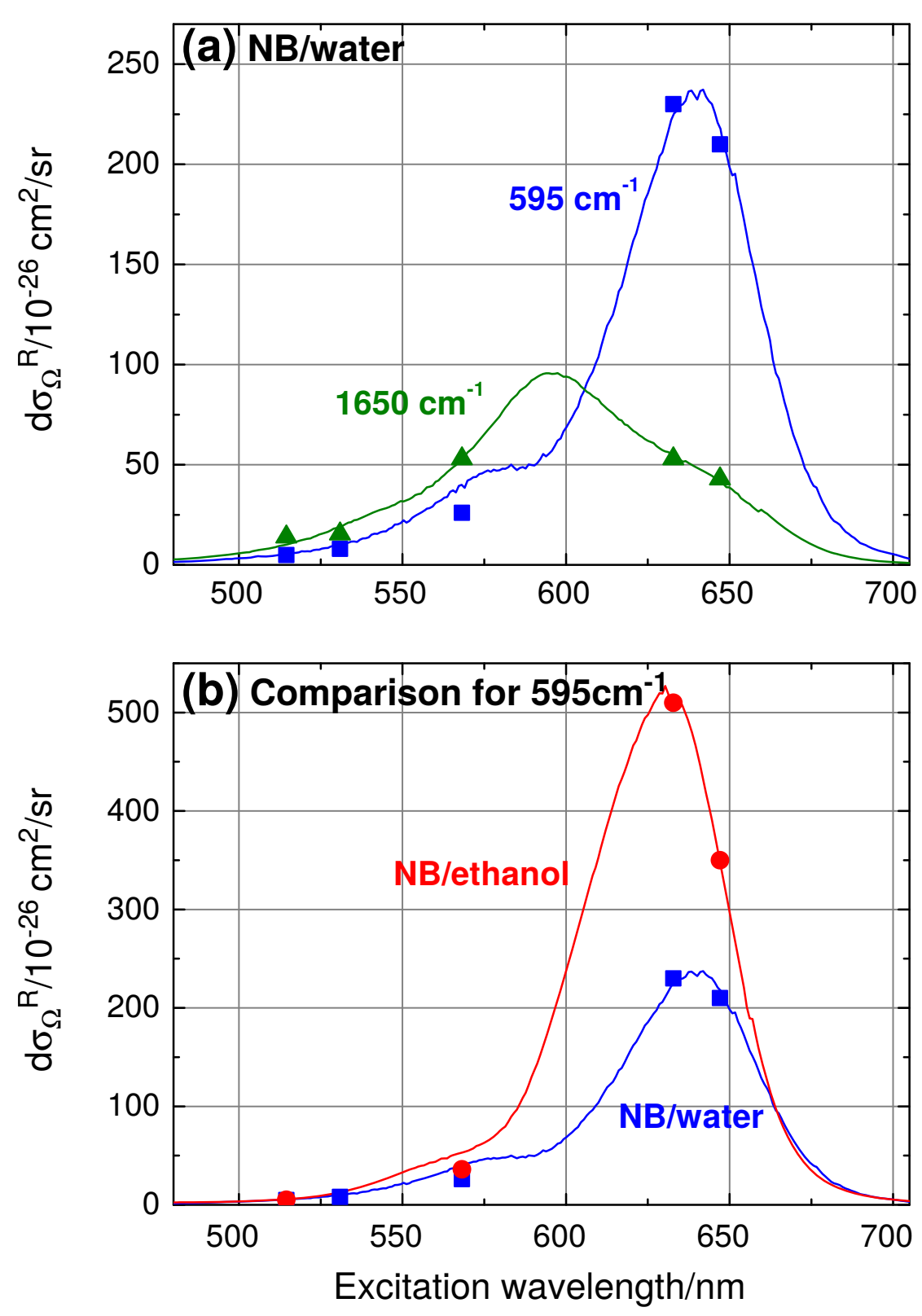

Figure 6.2: Resonance excitation profiles of the the $595 \mathrm{~cm}^{-1}$ and $1650 \mathrm{~cm}^{-1}$ modes of Nile Blue in water (a) and ethanol (b) obtained through the shifted-grating method. The solid lines are the excitation profiles computed from the optical transform model of the electronic absorbance as described in [146]. Symbols are the experimentally measured Raman cross sections at the given wavelengths. Reproduced with permission from [81]. 


$$
\varphi\left(\bar{\nu}_{L}\right)=P \int_{0}^{\infty} \frac{\sigma_{a b s}(\bar{\nu})}{\bar{\nu}\left(\bar{\nu}-\bar{\nu}_{L}\right)} \mathrm{d} \bar{\nu}-i \pi \frac{\sigma_{a b s}(\bar{\nu})}{\bar{\nu}}
$$

as proposed in [146]. Here $P$ is the principle value of the integral (reminiscent of the Kramers-Kronig relations [63]) and, as described in [146], the Raman excitation profile can be obtained by inserting the measured absoprtion profile of the molecule, $\sigma_{a b s}(\bar{\nu})$, into Equation 6.8 that can then be used in Equation 6.7. While the full derivation of Equation 6.7 is particularly complicated and is discussed in detail in Reference [147], it nevertheless provides a useful tool for relating the measured optical absorption to the resonance Raman profile of a vibrational mode, and as seen for $\beta$-carotene [146] and Nile Blue [81], reproduces extremely well the measured REP. The relation is not strictly universal, where the original authors showed its failure for cyanocobalamin, but its validity has been verified in a number of studies shortly after its proposal $[148,149]$. To quote Anne Myers Kelly many years later [145]:

The resonance Raman excitation profile, which is a plot of the cross-section for a particular Raman transition as a fucntion of the excitation frequency $\omega_{L}$, often tracks closely with the absorption spectrum but is not identical to it and may, in some cases, have a very different shape.

Given this relation, it follows that if the absorbance of the molecule is perturbed or modified by the presence of the metal nanostructure then the REP would be modified in turn. The idea is depicted in Figure 6.1 whereby the energy levels (here $\mathrm{S} 0 \rightarrow \mathrm{S}^{*}$ for simplicity) of the molecule can shift in wavelength because of adsorption to the metal surface. As such, if Equation 6.7 is still valid for the molecule on the metal then the wavelength dependence of the molecules Raman cross section will be modified.

This phenomenon was investigated by Lombardi et. al [139] using a tunable excitation source. This study found that different Raman modes of copper phthalocyanine, a molecule commonly used as a donor in photovoltaic cells, showed significantly different Raman Excitation Profiles for totally symmetric 


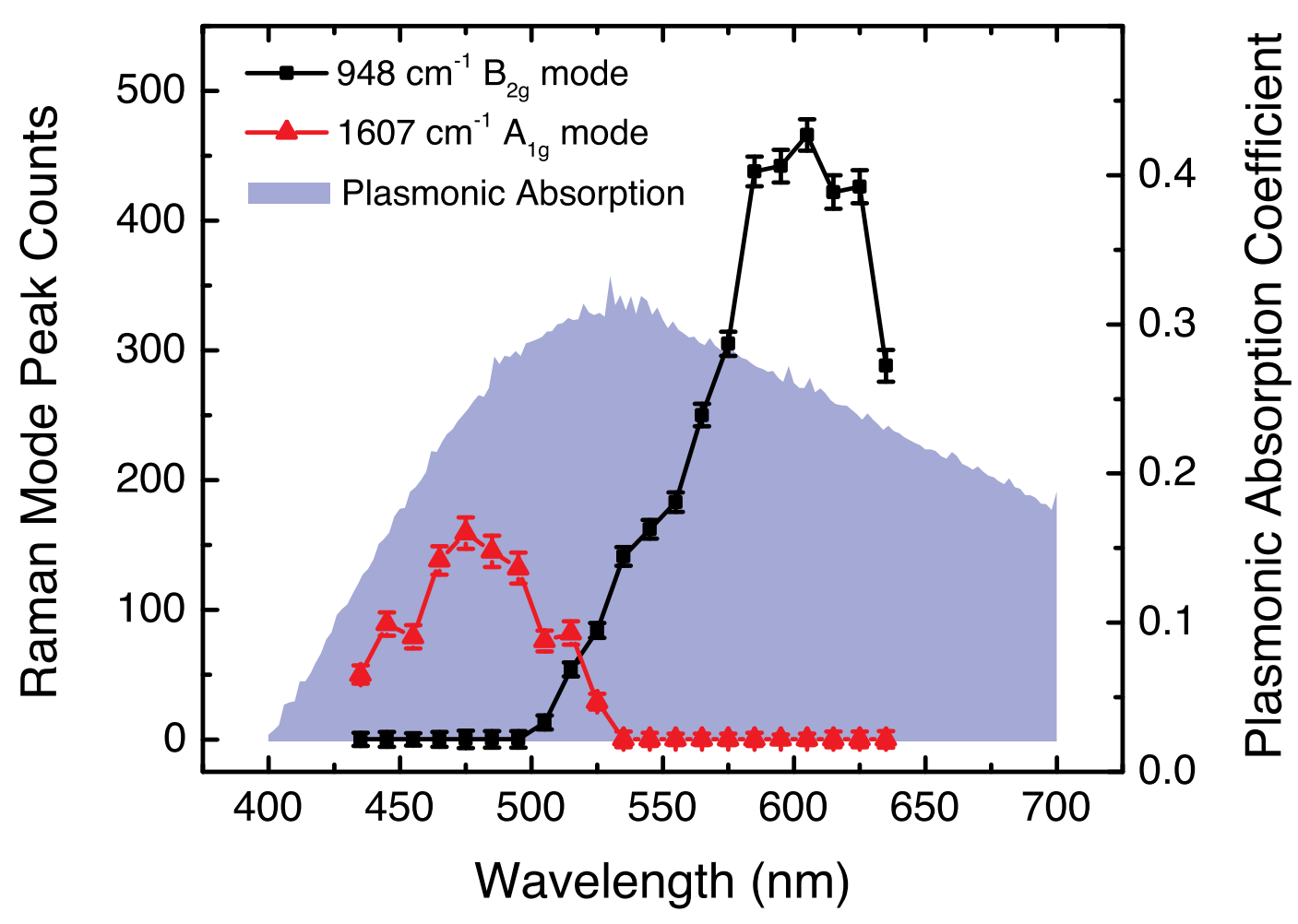

Figure 6.3: Resonance excitation profiles of the the $1607 \mathrm{~cm}^{-1}$ and $948 \mathrm{~cm}^{-1}$ modes of $\mathrm{CuPc}$ adsorbed on silver island films, with both peaks showing a significantly different wavelegth dependent intensity. Figure reproduced with permission from [139]. 
$\left(A_{1 g}\right)$ modes and non-totally symmetric $\left(B_{1 g}\right.$ and $\left.B_{2 g}\right)$ modes [54]. This was attributed to the presence charge transfer resonances whereby through HerzbergTeller selection rules [144], it was shown that the non-totally symmetric modes are enhanced through intensity borrowing from an inferred charge transfer state. The results from this study are shown in Figure 6.3. These interpretations are an indirect indication of changes in the electronic states of the molecule and rely on complicated theoretical treatments of the molecule-metal system developed by Lombardi et al. [144]. There is no direct evidence of charge transfer states in the optical (UV-Vis) properties of the dye-metal structure and this approach does not correctly account for the variation on local field intensities due to the plasmon dispersion of the SERS substrate. Such studies, although interesting, further highlight the difficulty in fully elucidating the mechanisms in Resonant Raman profiles in SERS, and the need for a method to directly observe the optical properties of the isolated dye molecule on the metal surface is clear. In Section 6.4.2 the modified absorption spectrum of Crystal Violet, as measured on single Ag spheres, is correlated with the SERS REP on the same Ag spheres to show that the resonance profile of the Raman modes of Crystal Violet are indeed modified from that in solution, providing definitive evidence for a modification of the electronic resonance of the molecule.

\subsubsection{Strong Coupling between Plasmons and Molecules}

There is an intense ongoing research effort to pin down the mechanisms governing the interaction between the resonances of adsorbed molecules and the underlying plasmon resonance of the substrate [150-152]. Such interactions, generally referred to as "plasmon-molecule coupling" or "strong-coupling", are concerned with how the resonances of both the adsorbed molecules and the plasmonic substrate can couple together and consequently produce a moleculeparticle system that has distinctly modified optical properties than that of its counterparts. Understanding the coupling between molecule and plasmon resonances is of great interest from fundamental electromagnetic and surface enhanced spectroscopy points of view but also provides a basis from which exotic "hybrid" molecular-plasmonic materials can be developed for new applications. 
There has been a vast amount of work in the past few years, both theoretical and experimental, dedicated to studying molecule-plasmon interactions. The majority of these studies focus on the idea of a splitting of the plasmon resonance when the molecular and plasmon resonance energies overlap. This observation is often thought of as being analogous to Rabi splitting [153], a phenomenon observed in systems where coupled resonances exist and explains the use of the term "strong coupling" to describe the regime where this splitting occurs. Such splitting was first observed [154] on nanoparticles when the transmission spectrum of a metal film coated with dyes was found to broaden and split with respect to that of the bare film. From here, many other studies have attempted to go further in describing this splitting phenomenon and numerous theories have emerged to predict experimental observations. Van Duyne and co workers [155], began to systematically investigate the effects resonant molecules adsorbed on metal nanoparticles have on the LSPR and found that the shift in LSPR due to adsorbates is strongly dependent on the relative wavelength of the molecular and plasmon resonance. Further studies by the Van Duyne group [156] also found that the shift in LSPR was strongly dependent on the concentration of adsorbed dyes, indicating that dye aggregates were forming on the surface of the nanostructures used and that these $\mathrm{J}$ and $\mathrm{H}$ aggregates play a role in the overall absorption spectrum of the dye-plasmon system. Ni et al. [157] again observed an anti-crossing behaviour of the LSPR for colloidal Au nanorods of varying plasmon resonances coated with HITC dye when the plasmon and molecular resonance overlap. This study confirmed the dependence of plasmon shift on dye concentration and rod-dye spacing. $\mathrm{Ni}$ et al. extended their studies to a range of positively charged dyes [158] and improved upon the ensemble nature by using dark-field scattering [159] to probe the properties of single Au nanorods coated with HITC and found that the overall scattering spectrum of the dye-rod system exhibits three distinct peaks when the plasmon lies between the dyes two resonances, in comparison with the standard splitting observations. Since then the number of papers studying plasmon molecule interactions has steadily increased. The Rabi-type splitting often observed in such studies is depicted in Figure 6.4.

Despite the extensive theoretical work devoted to understanding plasmonmolecule coupling, ranging from quasi-static approximations [160] for dye coated spheroids, finite-differnce-time-domain approaches [161] to quantum 

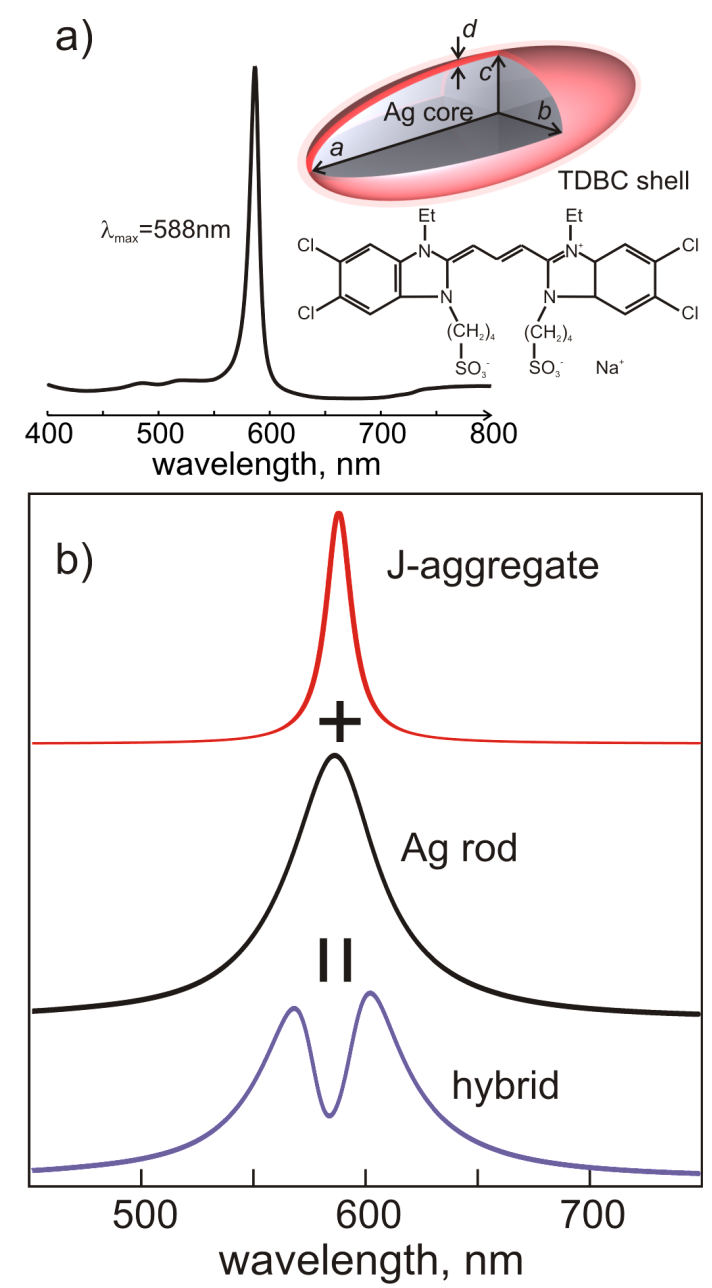

Figure 6.4: Schematic depiction of typically observed splitting of the plasmon resonance of a TBDC-coated gold nanorod, whose plasmon resonance position overlaps with that of the J-aggregate resonance, i.e. the strong coupling regime. Reprinted from Ref [151] 
mechanical treatments [40], experimental findings seem to often be at odds with theory depending on the system being studied. One can find reports of contradictory observations (scattering dips, assymetric line shapes, excitonic and plasmonic splitting [162]) and the need for a better understanding of the mechanisms at play is clear. One consideration that may break the deadlock of understanding these complex interacting systems is to measure the intrinsic effect (chemical and/or electromagnetic) of the NP on an isolated adsorbed molecule. This question is not directly addressed in the great number of recent studies devoted to the topic of strong-coupling between plasmons and molecules $[150,151,155,156,158,159,161-169]$; in such a strongly interacting system the molecular response cannot be isolated. Moreover, in such studies the dye concentration is often large (typically monolayer coverage and above) to maximize dye-plasmon interactions. Dye-dye interactions cannot therefore be neglected and are expected to induce resonance shifts of the dye layer independently of any plasmonic effects; in fact many studies specifically work with $J$-aggregates rather than isolated dyes $[150,151,158,159,163,164]$ whose properties are by definition determined by the coupling between molecules.

Furthermore, one of the drawbacks of these approaches is when modelling the dye-nanoparticle system, the dye-layer optical properties are generally assumed to be the same as that in solution, i.e. a single or double Lorentzian lineshape with peak positions corresponding to that measured in solution. There are no mentions of models that explicitly allow for shifts and or modifications of the dye properties before computing the interaction with the plasmonic core particle. This point is further strengthened by the fact that many studies use plasmonic substrates that are either fabricated on surfaces [170] or drop-cast from solution on flat surfaces $[151,159]$, i.e the surrounding medium is air. As such, the dye molecule can no longer be considered to be in the same environment as in solution, which may inherently change it's properties. These aspects work to further complicate elucidating the system properties, in particular obtaining the true dye absorbance on the metal surface. To this end, working with colloidal solutions of nanoparticles as the plasmon supporting substrate, as is the approach used in this chapter, removes many of the problems associated with using dried structures in investigating plasmon-molecule interactions. 


\subsection{The Challenge of Measuring Dye Surface Absorbance}

Observing surface enhanced and/or modified absorbance of dye molecules on nanoparticles has up until now proven elusive because of the difficulty in performing such an experiment. A natural approach to retrieve the absorbance spectrum of the dyes would consist of measuring the dye-plus-nanoparticle solution and then subtracting a reference solution of bare nanoparticles without dyes as illustrated in Figure 6.2.

This approach however turns out to be naive for a number of reasons, some more obvious than others. The experimental challenge comes down essentially to three points:

1. The optical cross sections of nanoparticles is often much larger than that of dye molecules. This makes measuring the dye absorbance in a solution of colloids an extremely difficult task because referencing becomes a serious issue.

2. Using differential extinction to extract the dye resonance, as would be the case with standard transmission setups, turns out to introduce artifacts in the measured dye spectrum due to minor shifts in the underlying plasmon resonance.

3. Sub-monolayer concentrations of dyes must be used such that the dye coverage of nanoparticles is low enough that dye-dye interactions do not influence the measured absorbance spectrum. This exacerbates point one putting an upper limit on the dye signal that can be measured using standard techniques.

These points are addressed in detail below. 

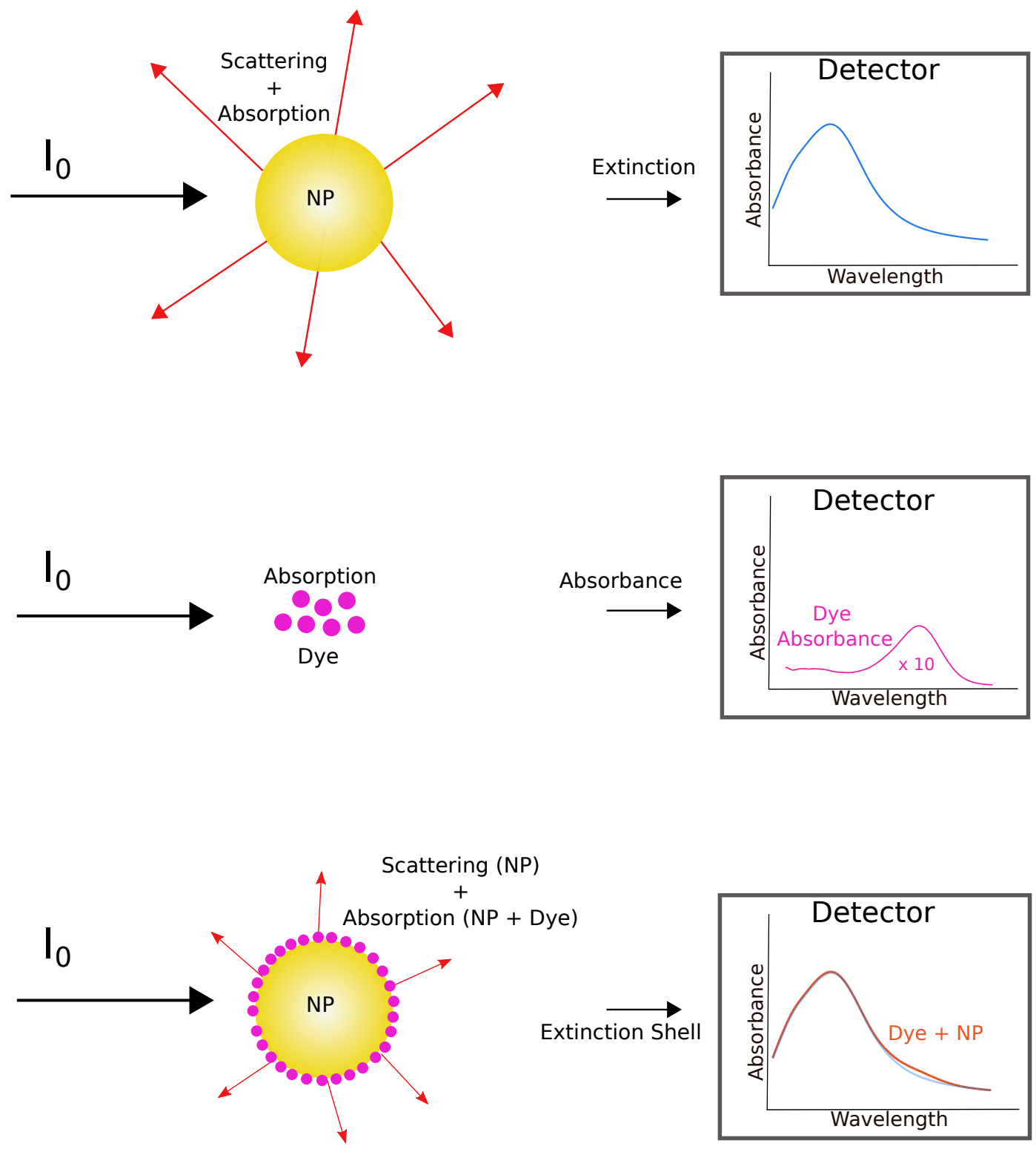

Figure 6.5: Schematic illustrating the idea of how the surface absorbance of dyes adsorbed to metallic nanoparticle in a standard extinction setup might be measured. The reference solution (top) is the bare nanoparticles, that both absorb and scatter light, measured as the extinction spectrum on the right. Dye molecules (middle) have neglible scattering, and thus only display an absorption spectrum. The sample solution would then be nanoparticles with a layer of dye molecules adsorbed (bottom) whose extinction spectrum contains the scattering and absorption of both the nanoparticle and the dye layer, which is almost identical to the bare nanoparticle. A naive approach would be to subtract the top spectrum reference from the coated nanoparticle to extract the dye spectrum on the surface. 


\subsubsection{Nanoparticle vs Dye Cross Sections}

Point 1 is illustrated in Figure 6.2.1 (a) where the extinction spectrum spectra of solutions of Nanocomposix and Lee and Meisel colloids at one-quarter concentration are plotted along with the absorbance spectrum of a $10 \mathrm{nM}$ solution of Rhodamine 6G, measured in the same UV-Vis (transmission) setup. Figure 1 highlights the significant difference in optical cross sections between nanoparticles and dyes, whereby the dye peak absorbance is $\sim 5-6$ orders of magnitude (normalised to concentration) less than that of the nanoparticle (at resonance). These results agree with theoretical predictions from Mie theory which predicts NP extinction cross section of $\sim 10^{-10} \mathrm{~cm}^{2}$ while most organic dyes like Rhodamine $6 \mathrm{G}$ and Crystal Violet have absorption cross sections of $\sim 10^{-16} \mathrm{~cm}^{2}$.
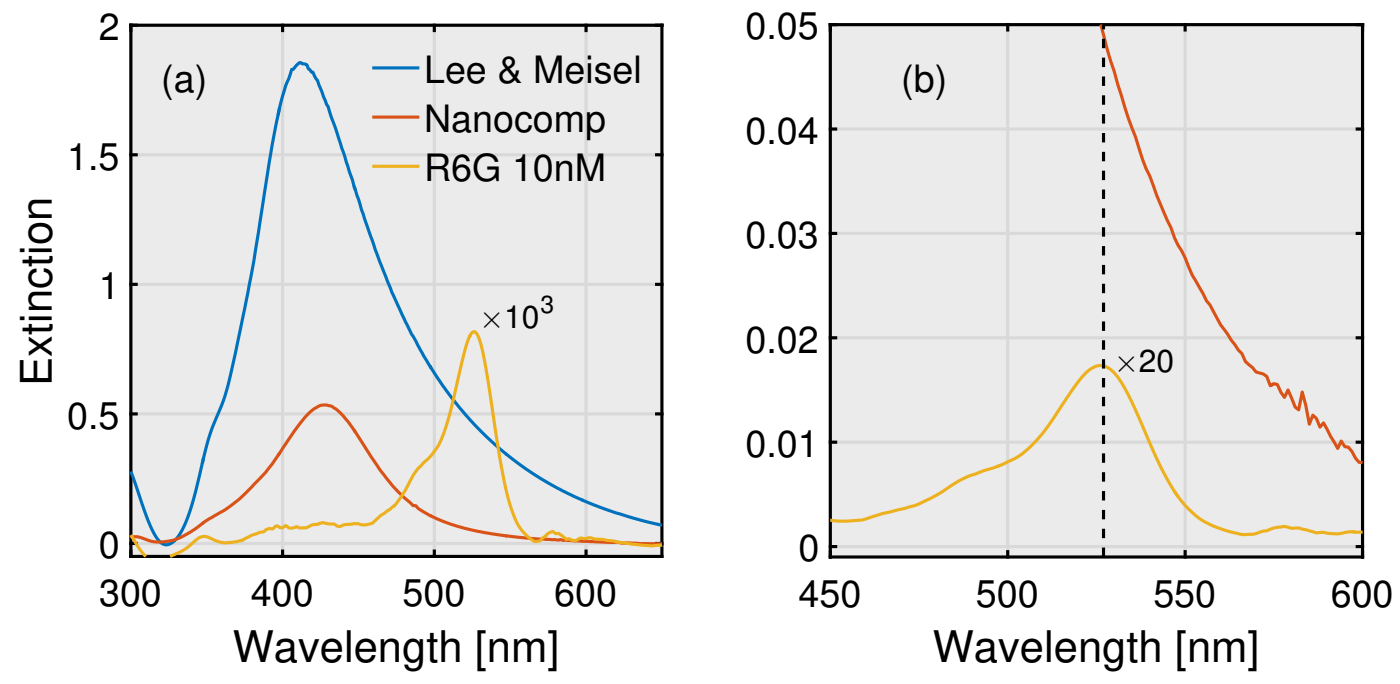

Figure 6.6: Illustration of the relative optical cross sections of dyes and Ag nanoparticles. (a) Extinction spectra of Lee and Meisel (blue) and Nanocomposix (red) colloids at one-quarter concentration, along with the absorption spectrum of Rhodamine $6 \mathrm{G}$ at $10 \mathrm{nM}$ in water (orange), scaled by a factor of 1000 for visualization. (b) Zoomed plot of Rhodamine 6G multiplied by 20 along with the extinction spectrum of Nanocomposix colloids. The dashed line indicates the absorption maximum of R6G at $527 \mathrm{~nm}$.

At the concentration used here for R6G, the peak absorbance is $\sim 10^{-3}$ at $\sim 527 \mathrm{~nm}$. In a standard, single beam UV-Vis spectrometer, the limit of detection is generally on the order of $10^{-3}$ absorbance units. A solution of $10 \mathrm{nM}$ of Rhodamine $6 \mathrm{G}$ is therefore approaching the limit of detection for 
standard UV-Vis spectrometers. At such a concentration, artifacts due to noise and reproducibility hamper the quality of the spectrum obtained, and great care needs to be taken when referencing to avoid background artifacts.

This is the first obstacle to measuring the surface dye absorbance and highlights the fact that standard UV-Vis setups are simply not sufficient to obtain accurate spectra that are not perturbed by background issues due to sample placement and referencing. The large background due to the colloids means that any small changes in the reference sample will feature in the absorption spectrum of the dye. For example at $527 \mathrm{~nm}$, the dye absorbance is $1 \times 10^{-3}$ (Figure 6.2.1 (b)) while the nanoparticle extinction is $\sim 0.05$. Thus even a $1 \%$ change in the reference solution would contribute an absorbance artifact of the order of $5 \times 10^{-4}$ to the measured dye signal. Minor changes such as this, which could easily arise from pipetting errors, sample placement etc, seriously hamper the ability to measure dye spectra in turbid solutions at nano-molar concentrations. By using an integrating sphere, these effects can be mitigated which will be discussed later.

As will be clear in the next section, for the colloids used in these studies, $10 \mathrm{nM}$ of dye is at the limit of the required concentrations to elucidate the true dye absorbance, pushing the sensitivity levels further outside the realm of standard UV-Vis spectrometers.

\subsubsection{Differential Extinction Artifacts}

To understand better the mechanisms governing the dye-nanoparticle system, Mie-theory [68] calculations are employed to predict the overall optical response of the dye coated nanoparticle. Modelling the dye-nanoparticle system reveals the importance of both working at low dye concentrations and using differential absorbance rather than extinction to extract the dye resonance successfully. The system is treated as a simple shell model where the dye is modeled as an effective medium dielectric layer surrounding the core Ag nanoparticle. The details of these calculations are described in Chapter 2 and Appendix A. All Mie-theory calculations were performed in Matlab using the SPlaC package [16] that calculates the Mie coefficients of a sphere under plane wave excitation from which the local electric fields and far field properties of 
the nanoparticle, dye and nanoparticle-dye ensemble can be computed. The reader is referred to Reference [16] for further details on how these calculations are implemented in Matlab.

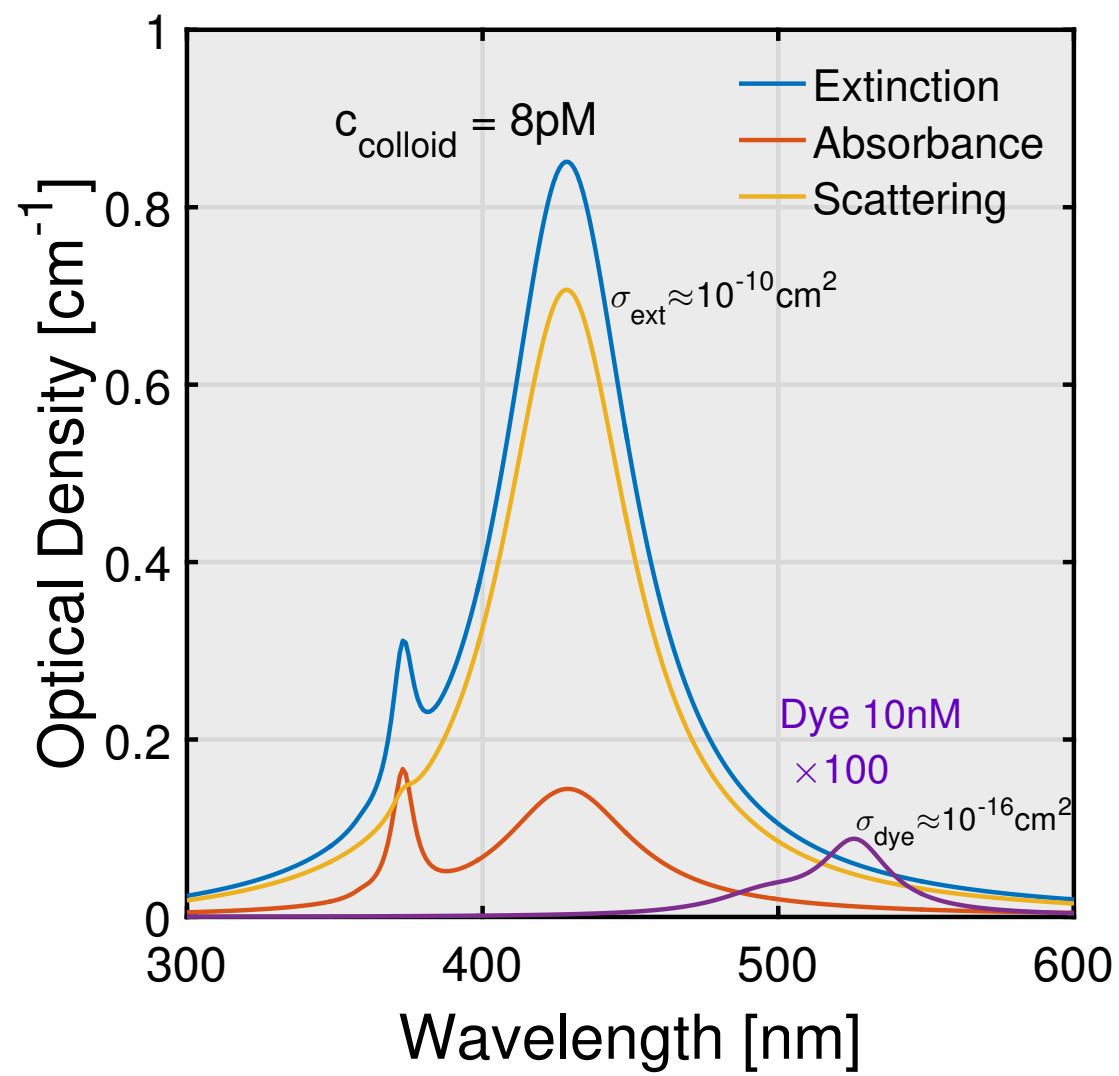

Figure 6.7: Extinction (blue), absorption (red), and scattering (orange) crosssections for a $30 \mathrm{~nm}$-radius silver nanosphere in water and absorption crosssection of a typical dye (black). Peak optical densities (OD) are indicated for concentrations of $8 \mathrm{pM}$ Ag-colloids and $10 \mathrm{nM}$ dye.

In Figure 6.7, the extinction, scattering and absorption spectra of a solution of $60 \mathrm{~nm}$ diameter silver nanospheres in water (at a concentration of $7 \mathrm{pM}$ corresponding to that used in experiment) as predicted by Mie theory are plotted along with the absorption spectrum of Rhodamine $6 \mathrm{G}$ at $10 \mathrm{nM}$ and scaled by a factor of $10^{3}$ for comparison. From the relative peak positions of the nanoparticle and dye resonances, it is clear that in the context of Section 6.1.3 the de-tuning is large $(>100 \mathrm{~nm})$ and no coupling between the plasmon resonance and the dye resonance is expected. As such, one would expect that extracting the dye layer absorbance simply requires subtraction of a reference solution of neat nanoparticles extinction from the dye-nanoparticle extinction as in a standard UV-Vis setup. 
This approach is however flawed as illustrated in Figure 6.8, where the difference spectra obtained by subtracting the response of a neat silver NP shown in Figure 6.7 from the same NP coated with a $1 \mathrm{~nm}$ shell of R6G dye. The dielectric function of the dye layer is obtained from the ClausiusMossotti relation (See Appendix A), using a dye density of $0.1 \mathrm{~nm}^{-3}$. Here, the predicted extinction difference spectrum departs substantially from the absorbance spectrum of the adsorbed dye, showing a negative difference within the tail of the plasmon resonance, strongly distorting the dye lineshape around $500 \mathrm{~nm}$. This is a consequence of the minute shift of the plasmon resonance due to the adsorbed dye layer. Shifts in plasmon resonances due to adsorbed layers have been well studied [155]. The shift in plasmon resonance arises because of a minute change in the refractive index (with respect to the nanoparticle solvent, here water) surrounding the particle when the molecular layer is present. This change is almost unnoticeable experimentally in the raw extinction spectrum, but when the extinction difference is taken (blue curve, Figure 6.8, the size of this shift becomes comparable to the absorption of the dye layer and thus prevents extracting a meaningful spectrum for the dye layer. While the shift in plasmon resonance can be used to quantify the amount of adsorbed analyte, the basis of LSPR sensing [171-173], down to the picomolar range [174] of biomolecules, the effect is problematic for measuring the properties of the analyte itself.

Interestingly, by working with differential absorbance one can mitigate the artifacts present in the differential extinction tail. This is seen in Figure 6.8 (red solid line) where the differential absorbance $\delta \sigma_{a b s}$ spectrum much more closely resembles the original dye spectrum in solution without negative peaks due to the LSPR shift. This arises from the fact that the absorption cross section is for a $60 \mathrm{~nm}$ silver sphere is about 6 times smaller at resonance than the extinction cross section as seen in Figure 6.7 (a).

An illustration of the effect of refractive index on the differential absorbance and extinction spectra is easily shown by considering the predicted spectra for $60 \mathrm{~nm}$ diameter silver spheres (again at concentration $8 \mathrm{pM}$ ) embedded in water (refractive index, $n=1.33$, or $\epsilon_{\mathrm{M}}=n^{2}=1.77$ ) and in a medium of slightly lower $n$, giving $\epsilon_{\mathrm{M}}=1.74$. The extinction and absorption spectra for such configurations are plotted in Figure 6.9 (a), where the effect of the surrounding medium only constitutes a minor blue-shift shift in the peak plasmon position 


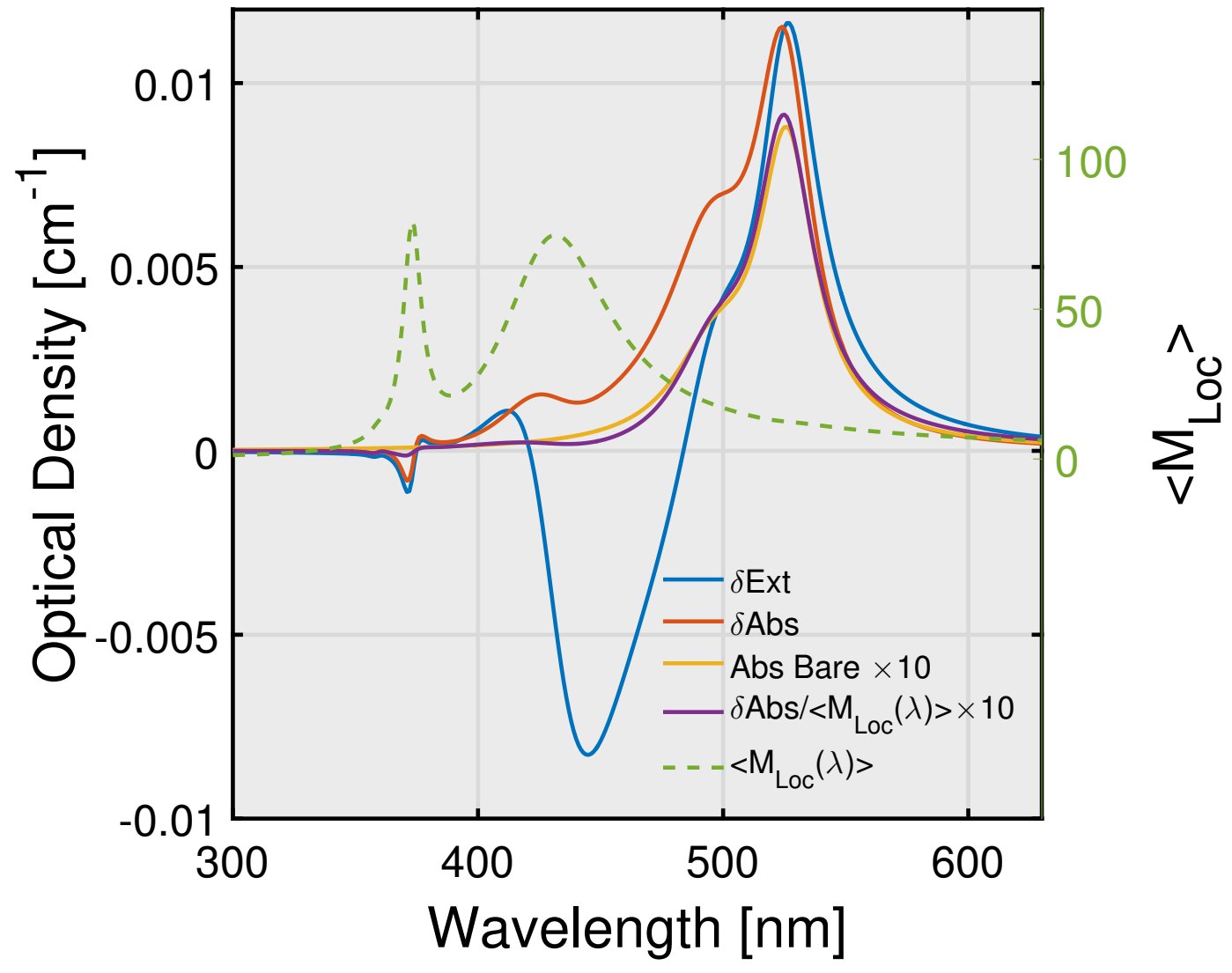

Figure 6.8: Difference spectra obtained by subtracting the response of the bare sphere shown in Figure 6.7 from the same colloid coated with a $1 \mathrm{~nm}$ dye-shell (concentration $0.1 \mathrm{~nm}^{-3}$ ). The dashed green line corresponds to $\left\langle M_{\text {loc }}(\lambda)\right\rangle$, the surface-averaged field intensity enhancement factor (rightaxis scale). The corrected differential absorbance $\delta \sigma_{\text {abs }}(\lambda) /\left\langle M_{\text {loc }}(\lambda)\right\rangle$, matches closely the reference dye absorbance. 
and slight increase in intensity for the lower $\epsilon_{\mathrm{M}}$. When one plots the differential extinction and absorbance however (Figure $6.9(\mathrm{~b})$ ), where the particles water response is subtracted from the lower index response, the induced shift becomes apparent as a peak in the $410 \mathrm{~nm}$ region and a negative dip in the 450 to $500 \mathrm{~nm}$ region. Plotted also is the absorption spectrum of RH6G at $10 \mathrm{nM}$ concentration (scaled by a factor of ten for visualization). It is immediately clear that the induced shifts due to refractive index change around the particle introduce spectral features (in the extinction spectra) that are larger in magnitude than the dye response at this concentration, and will interfere with the background when extracting the dye spectrum. Again, as argued in Figure 6.8, the effect is less pronounced (by a factor of about 6) when the differential absorbance is computed, simply due to its relatively lower cross section as compared to extinction.

Despite the crude nature of the above example, it sufficiently demonstrates the unavoidable effects that the plasmon resonance can have on the surface dye spectrum. One could argue that using a single value for the refractive index change to investigate the effect the dye layer has on the LSPR is too simple because dye molecules clearly have a complex dielectric function that is wavelength dependent. However in the region of interest for the nanoparticles used $(430 \mathrm{~nm})$ the absorption of most dyes used is close to zero so the imaginary part of the dielectric function is close to zero here and only the real part of $\epsilon$ affects the plasmon resonance position. In Figure 6.9 (c), the dielectric function of Rhodamine $6 \mathrm{G}$ obtained by the Clausius-Mossotti formalism (here fitted with a double Lorentzian profile) outlined in Appendix A is plotted. While the imaginary part of $\epsilon$ is close to zero at $430 \mathrm{~nm}$, the real part is 1.74, explaining why this value was chosen. In fact more crucial to modelling correctly the plasmon shift is the value used in Appendix A for the molecules static polarizability, $\alpha_{\text {static }}$ which is found from DFT calculations (see Appendix A) in the long wavelength (DC) limit that gives the molecules (real) response far from any resonances $[16,175]$.

It is important to note that the observed effects are specific to the nanoparticlemolecule system studied here and such phenomena may be more or less important depending on factors such as the nanoparticle size, dye optical properties and the relative peak positions of plasmon and molecular resonances. In particular as the nanoparticle size increases, scattering intensity may drop due to 

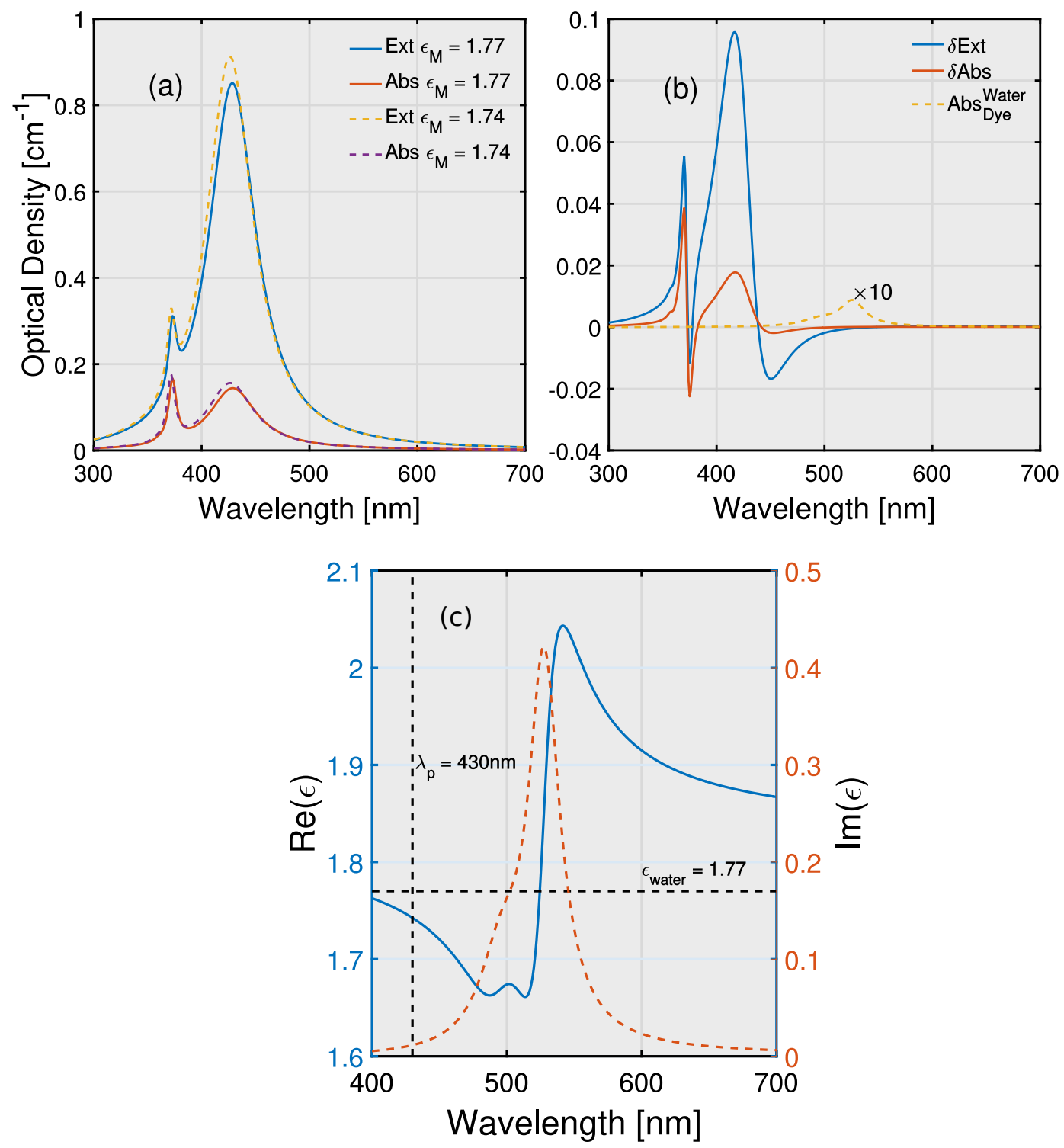

Figure 6.9: (Effect of the surrounding dielectric medium on the plasmon resonance of a silver nanosphere: (a) Extinction and absoprtion spectra of a $30 \mathrm{~nm}$ radius $\mathrm{Ag}$ sphere in a surrounding medium of (real) dielectric constant 1.77 (solid lines) and 1.74 (dashed lines). (b) Differential extinction and absorbance spectra obtained by subtracting the $\epsilon_{M}=1.74$ sphere response from the $\epsilon_{M}=1.77$ response. The dashed orange line is the absorption spectrum of Rhodamine 6G at $10 \mathrm{nM}$. (c) Real (blue) and imaginary (dashed-red) parts of the dielectric function of Rhodamine $6 \mathrm{G}$ in water obtained through the Clausius-Mossotti relation from a Voigt-fit to the optical absorption as outlined in Appendix A. 
radiation retardation effects. The arguments presented are however sufficient for the purposes of the system under investigation.

With these considerations in mind, to probe potential chemical and electromagnetic effects of dye-metal interactions it is clear that one should work with differential absorbance rather than extinction to correctly extract the dye spectrum independent of plasmon effects. This moreover reduces the optical signal of the NPs (by a factor of 4 to 6 here) without affecting that of the dye for which the scattering component is negligible. As seen in Figure 6.8, the differential absorbance spectrum is not exactly the same as that of the bare dye, but is modified/enhanced by the surface-averaged local field intensity enhancement factor $\left\langle M_{\mathrm{Loc}}(\lambda)\right\rangle$ associated with the plasmon resonance. This effect is still important relatively far from the resonance as shown in Fig 6.8 where the $\left\langle M_{\text {Loc }}(\lambda)\right\rangle$ spectrum (dashed green line) is superimposed under the dye spectrum. As a result, for a dye with multiple resonance peaks and a non-neglible linewidth, the relative intensity of the peaks is predicted to change because of the wavelength-dependence of this enhancement factor. This is evident in Fig 6.8 for a typical dye absorbance with a vibronic shoulder where the shorter wavelength peak is more enhanced than the main electronic resonance. For well-defined NPs however this plasmonic enhancement can be predicted and the differential absorbance spectrum corrected accordingly by normalization of $\left\langle M_{\mathrm{Loc}}(\lambda)\right\rangle$. This is evident in Figure 6.8 where the corrected dye spectrum (solid purple line) resembles closely the bare dye in solution.

\subsubsection{Concentration Dependence of Dye Layer Proper- ties}

The discussion has so far focused on a low dye concentration of $\sim 0.1 \mathrm{dye} / \mathrm{nm}^{2}$, but in many of the previous studies dye concentrations have instead been large, typically at or beyond monolayer coverage. Figures $6.10(\mathrm{a}-\mathrm{b})$ illustrate how dye-dye interaction effects may then interfere with the measurement of the intrinsic absorbance of the adsorbed dye. In this example calculations, the dye surface coverage is increased towards a typical monolayer coverage of one dye $/ \mathrm{nm}^{2}$. At such short distances, the interaction between dyes needs to be included in the effective medium properties of the coating layer, which we 
describe via the Clausius-Mossotti equation, assuming a uniform shell thickness of $1 \mathrm{~nm}$ for the sake of argument.

Two configurations are considered to disentangle the effect of the NPs with the intrinsic dye-dye interactions in the dye layer: the dye-coated silver nanosphere as before (b), and the same shell with the silver replaced by water (a). Below concentrations of $0.1 \mathrm{dye} / \mathrm{nm}^{3}$ in the shell, local field (ClausiusMossotti) corrections are negligible and the differential absorbance spectrum matches the spectrum of the free dye in solution enhanced by the plasmonic field enhancement factor (dashed red line). As the dye concentration increases, the dye-resonance splits into two resonances as predicted from the standard exciton model [176]: the blueshifted resonance is associated with the side-byside dipolar interaction between dyes, while the redshifted one corresponds to head-to-tail dipoles. Both resonances are excited in a spherical shell as shown in the surface-field diagram inset in (a).

The same type of interactions are expected for Ag@dye core-shell structure, but the surface field is then mostly perpendicular to the surface (see inset in (b)), thereby strongly favoring the blue-shifted (side-by-side) resonance. The inset presents the strength and orientation of the local electric field on the surface of the metal, at a wavelength of $550 \mathrm{~nm}$. The polarization is approximately linear, and largely normal to the metal surface due to the strong contrast in dielectric function with the surrounding medium. As a result, dipole coupling is more strongly enhanced for parallel dipoles, rather than head-to-tail. Such effects were considered early on when the discovery of SERS was prompting investigation into dye-coated metallic nanoparticles [177] after the first observations of dye-plasmon interactions [154].

These predictions highlight the importance of dye-dye interactions effects in dye layers, even at sub-monolayer coverages. In order to measure the true surface-absorbance of the dye, it is necessary to exclude those by working at low concentrations. As a practical limitation, for the colloids used in these studies, a dye coverage of $0.1 \mathrm{dye} / \mathrm{nm}^{3}$ on $60 \mathrm{~nm}$ diameter spheres of concentration $8 \mathrm{pM}$ corresponds to a dye concentration of roughly $10 \mathrm{nM}$. It is possible that concentrations lower than this are required depending on the properties of the dye being used in terms of how it organises on the surface of the particle. 


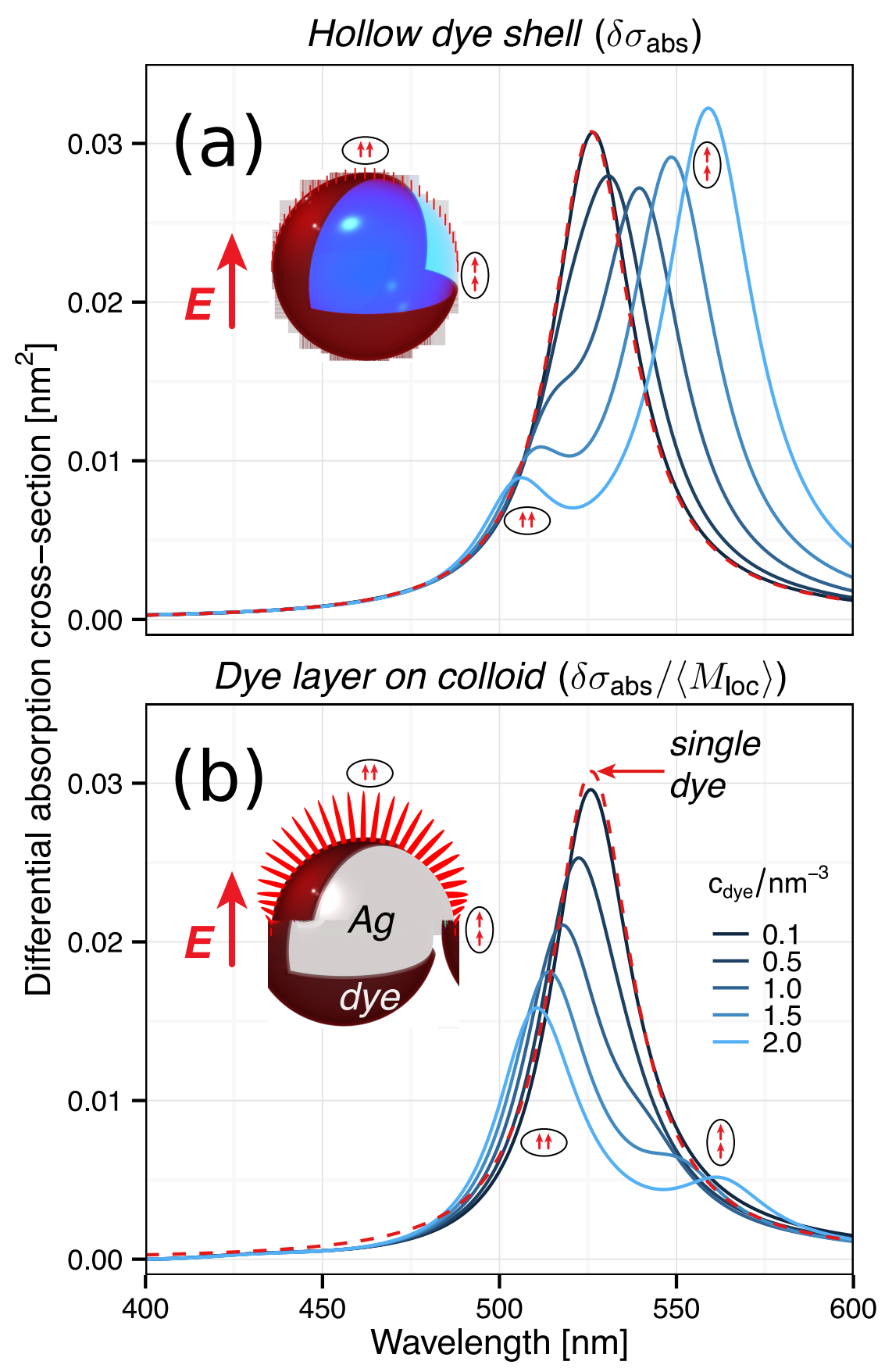

Figure 6.10: Differential absorption spectra as a function of dye concentration (from 0.1 to $2.0 \mathrm{~nm}^{-3}$ ) in the shell (normalised by $\left\langle M_{\text {Loc }}\right\rangle$ ). A single Lorentzian resonance at $526 \mathrm{~nm}$ is used here to highlight peak shifts, see reference dye spectrum (dashed red lines). and reference dye-spectrum (red dashed-line). The fictitious hollow shell with the NP replaced by water (a) is used as a toy-model to understand the case of a dye-layer on an Ag-sphere (b). The spectra in (a-b) split into two resonances associated with side-by-side and head-to-tail dipolar interactions, as dye-dye interactions become important. The insets show the polarisation ellipses of the local electric field (at $530 \mathrm{~nm}$ ). This figure was produced by Dr Baptiste Auguié for Ref [128]. 
The Mie-theory framework for modelling the dye-nanoparticle system is now established such that experimental results can be understood and compared to predicted values for both shifts and enhancements in the dye absorbance spectrum. Crucially it has been shown that a large detuning of the plasmon and dye resonances, use of differential absorbance spectra and sub-monolayer dye concentrations are all required in order to extract the dye resonance on the surface of the nanoparticle.

\subsection{Experimental Results}

\subsubsection{Sample Preparation}

All colloid samples were prepared according to the guidelines outlined in [92]. Each set of measurements requires a reference solution. Reference samples were prepared by first mixing $500 \mu \mathrm{L}$ of Ag colloid solution with $500 \mu \mathrm{L}$ of $2 \mathrm{mM} \mathrm{KCl}$ to displace the citrate capping layer (without inducing aggregation [92]). This solution was then mixed with $1 \mathrm{~mL}$ of MilliQ water and the resulting $2 \mathrm{~mL}$ (with a final colloid concentration of $8 \mathrm{pM}$ ) was pipetted into a cylindrical glass tube. The same procedure was performed for dye-colloid samples but replacing the water in the final dilution by twice the desired concentration of dye. For each concentration series, the same vial was used to ensure the exact same vial geometry for all measurements and concentrations were tested going from reference (no dye) to the highest dye concentration sequentially. Vials were cleaned with aqua regia prior to measuring and were rinsed thoroughly with water and ethanol after each sample was measured.

Note that this method relies on the assumption that the addition of dyes (which are charged) to colloids does not induce any intrinsic change in the colloid, in particular aggregation. Were aggregation present in the dye-colloid sample, referencing with bare colloids would no longer be valid. For the dye concentrations and the type of colloids used here, aggregation is not expected and would be evident in the appearance of a longer wavelength absorption peak associated with NP aggregates which is not present in the spectra measured.

Because the dye absorbance spectra are so small in comparison to the colloid spectra, great care was taken to ensure reproducibility between reference 
(colloids $+\mathrm{KCl}$ ) and sample solutions (colloids $+\mathrm{KCl}+$ dyes) and as such sample preparation details are crucial. To mitigate any preparation errors, the following guidelines were adhered to during all sample preparation:

- For concentration series, dye solutions were prepared on the day of experiments from a stock solution of generally $100 \mu \mathrm{M}$. A small volume of stock solution was poured out from which each dye concentration was prepared. Using RH6G as an example, a $100 \mu \mathrm{M}$ aqueous solution was diluted in steps of 10 down to $100 \mathrm{nM}$ (in volumes of $1 \mathrm{~mL}$ ). This solution was then used to prepare $1 \mathrm{~mL}$ of twice the final concentration needed for the concentration series. Each final sample was then mixed with colloids $+\mathrm{KCl}$ as described above.

- During dilution steps, it was ensured that both the user and the pipette tips were grounded to reduce any errors introduced by electrostatic sticking of dyes to the tips. This was achieved by holding a grounded conductor while performing dilutions and grounding pipette tips prior to pipetting dyes.

- For each sample, colloid stock solutions were taken from the fridge (stored at $4^{\circ} \mathrm{C}$ ) just before sample preparation, be it reference or sample solutions to ensure temperature variations were minimised. Samples prepared from colloids sitting on the bench for extended periods suffered from background issues.

- For bare dye in water measurements in the sphere, glass pipettes were avoided for introducing the sample to the CMCIS sample holder as it was found dyes (anionic or cationic) had a tendency to stick to the pipette walls and cause errors in measurements.

- While the absorbance signals measured in the CMCIS are generally stable over the space of 30 minutes to 1 hour, in concentration series the vial gets progressively dirtier (ethanol does not fully remove Ag colloids) and there is a limit to how many samples can be measured with the one vial before backgrounds become an issue. Mitigating this effect is done by simply preparing another reference solution midway through the series which was shown to improve backgrounds. 
- The halogen lamp was turned on at least one hour prior to measurements to ensure the temperature stabilised at 100W output. Similar considerations were used to ensure CCD drift in the spectrometer was minimised.

\subsubsection{Measuring Absorbance in a Turbid Medium}

An important consideration not addressed in Chapter 5 is whether the differential technique proposed actually yields the correct spectrum of the dye. Given the unconventional nature of the sphere geometry and the fact that the dyes are diluted in a turbid medium, it is certainly plausible that the measured dye spectrum becomes distorted spectrally from what would be measured in water. In fact there are numerous techniques whereby the scattering properties of the medium are utilised for spectroscopic applications such as spatially offset Raman spectroscopy (SORS) [178]. One could expect that the scattering of the Ag nanoparticles could lead to an increased path length of photons within the sample $[179,180]$, causing an increase in interaction length of light with dye molecules and thus a larger effective dye absorbance from that in water only. To test the effect of scattering on the measured dye absorbance,the dye molecule Eosin B was added to the silver colloids and its absorption spectrum measured in the integrating sphere. Eosin B is a negatively charged dye and does not readily adsorb to the negatively charged silver nanoparticle surface due to electrostatic repulsion (this can be easily demonstrated by the lack of SERS signal observed with Eosin, whereby the molecules experience no enhanced electric field due to lack of adsorption and thus no Raman signal is observed). For this reason Eosin B serves as the ideal candidate to test if the dye spectrum is distorted in the nanoparticle solution. Figure 6.11 shows the measured spectrum of Eosin B at $100 \mathrm{nM}$ in a solution of Nanocomposix colloids at one quarter concentration $(8 \mathrm{pM})$ and in water in normal UV-Vis transmission. After scaling the sphere absorbance by the sphere L-factor (2.4) the spectrum matches exactly that of the water spectrum, showing that for the concentration of colloids used, the turbidity does not induce any spectral changes in the dye absorbance.

The effects of scattering by the solvent medium on measured spectra within the sphere may not be valid for highly scattering or absorbing samples but in 


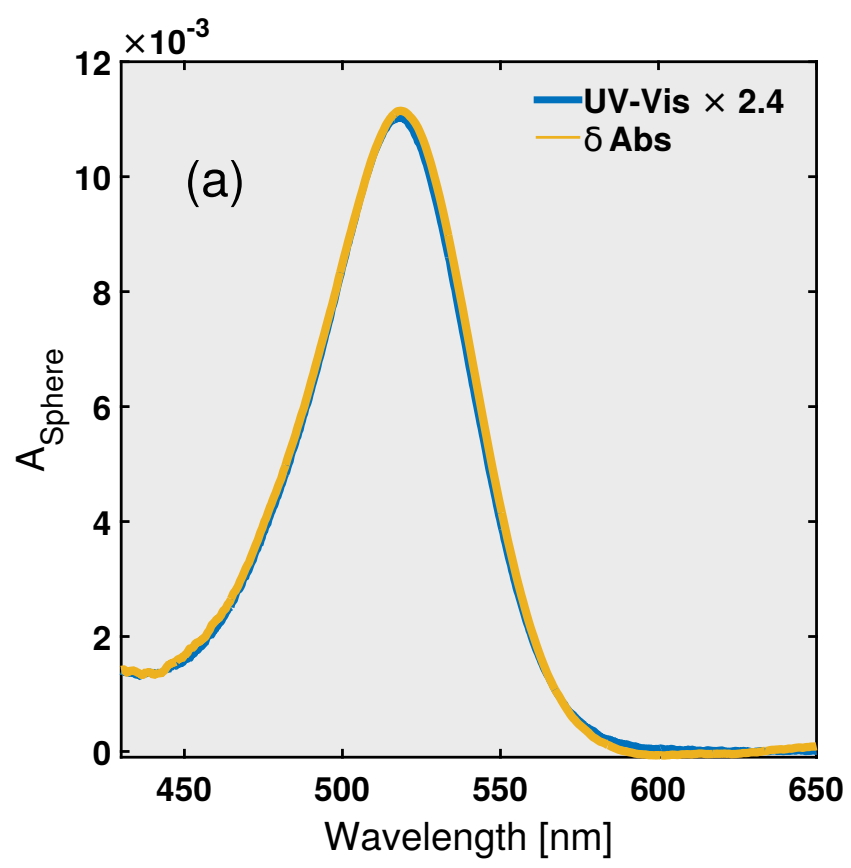

Figure 6.11: Investigation of the effect of scattering on the measured dye absorbances: The differential absorbance spectrum of the dye Eosin B at $100 \mathrm{nM}$ in Ag colloid solution (yellow curve) plotted against the same concentration of dye measured in a standard UV-Vis setup (multiplied by the sphere L-factor 2.4 ) with a $1 \mathrm{~cm}$ cuvette (blue curve). The differential absorbance spectrum matches almost exactly the spectrum in solution illustrating that the scattering component of the colloids does not influence the measured differential absorption spectra of dyes diluted in colloids. 
the case of the colloids used here, where the scattering signal in the $500-800 \mathrm{~nm}$ region is $\sim 0.1$, and the absorption of dyes is low, the effect is negligible. Ray tracing Monte-Carlo methods would need to be employed to correctly model the effect of scattering and absorbance on path length within the sphere as was done in Reference [122].

\subsubsection{Surface Absorbance Spectra}

This section presents the results achieved using the CMCIS setup for a range of commonly used dyes adsorbed to the surface of Ag colloids. All absorbance spectra presented are those obtained from the differential absorbance technique described in Chapter 5. The spectra have been post processed (smoothing, background removal) and corrected for the sphere path length enhancement $L_{\text {sphere }}$ and as such represent the equivalent absorbance that would be measured in a transmission configuration.

\subsubsection{Rhodamine 700}

The surface absorbance spectra of Rhodamine 700 (RH700) are first presented. RH700 was chosen because its main resonances are more than $200 \mathrm{~nm}$ from the plasmon resonance of the colloids, ensuring any coupling between the molecular and plasmon resonances will be negligible. Thus any modifications in the molecular absorbance can be solely attributed to the $\mathrm{Ag}$-molecule interaction and not due to plasmon effects.

Figure 6.12(a) shows the differential absorbance spectra of RH700 adsorbed on $\mathrm{Ag}$ NPs at three different concentrations (6, 12 and $24 \mathrm{nM}$ ). The reference absorbance spectrum of the dye in water (shown in grey) exhibits a main resonance at $642 \mathrm{~nm}$ with a vibronic shoulder at $593 \mathrm{~nm}$ (see Appendix A for details). Figure 6.12 (a) clearly demonstrates the ability of the integrating sphere approach to measure the surface absorbance of dyes on NPs at very low concentrations, down to $6 \mathrm{nM}$ here. The most dramatic observation is the clear redshift of the surface absorption spectrum by $\sim 20 \mathrm{~nm}$, with the main resonance now at $664 \mathrm{~nm}$ and the vibronic shoulder at $608 \mathrm{~nm}$. While a shift of a 


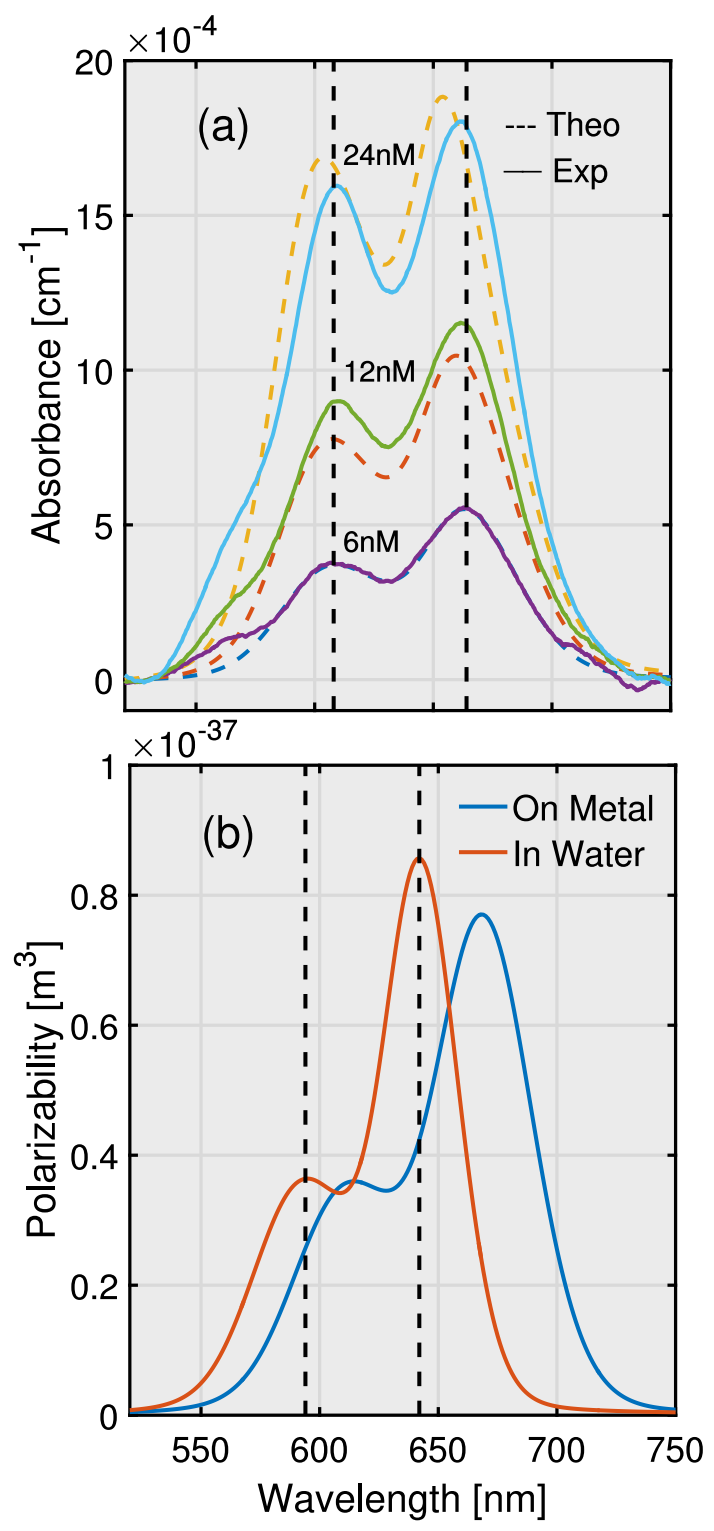

Figure 6.12: (a) Measured (solid lines) and predicted (dashed lines) differential absorbance spectra of Rhodamine 700 (RH700) at concentrations of 6, 12, and $24 \mathrm{nM}$ on $30 \mathrm{~nm}$-radius $\mathrm{Ag}$ nanospheres (8 pM). Those spectra are clearly red-shifted by $\sim 20 \mathrm{~nm}$ compared to the normal RH700 spectrum in water (shown in grey). Note a scaling factor of $\sim 5.3$ is necessary to quantitatively match experiments and theory (see discussion in the text). The modified surface polarizability of RH700 was modelled as a double-Voigt-type resonance (see Appendix A), with the same oscillator strength as bare RH700 and parameters adjusted to fit the experimental surface absorbance spectrum measured at $6 \mathrm{nM}$. The effective thickness of the shell was adjusted to $0.6 \mathrm{~nm}$. (b) Comparison between the deduced intrinsic surface absorption spectrum of RH700 and the reference bare RH700. 
1 or 3 nanometers with respect to the water absorbance might be attributable to many experimental errors (slight changes in colloid $\mathrm{pH}$ compared to water, wavelength dependence of sphere $M$ factor, background subtraction artifacts etc), a $20 \mathrm{~nm}$ shift constitutes a non-negligible change in the dye properties and must consequently indicate a change in the energy of the electronic transitions of the molecule. As explained earlier, this shift cannot to be due to the interaction with the plasmon resonance given the large detuning from the plasmon peak position $(430 \mathrm{~nm})$.

The effect of dye-dye interaction is in fact visible in the concentration dependence and results in a (very small) blue-shift of the resonances along with a change in relative intensity between the two peaks. As the concentration increases from $6 \mathrm{nM}$ to $24 \mathrm{nM}$, the intensity of the vibronic peak at $608 \mathrm{~nm}$ becomes larger in intensity than the main $\mathrm{S} 0 \rightarrow \mathrm{S} 1$ resonance, providing definitive evidence that the density of dyes on the colloid surface affects the average optical properties of the dye layer. These two features are in fact predicted by the theoretical electromagnetic model shown as dashed lines (see Section 6.4.1 for details). The observed red-shift at the lowest concentration must therefore be an intrinsic feature of the adsorbed dye and not a result of dye-dye interactions on the surface, while the emerging blue-shift with increasing concentration is predicted from the standard exciton model [176].

The measured modified absorbance can then be used to predict within the Mie-shell model the concentration dependence as shown in Figure 6.12 (a) (dashed lines). The concentration-dependent effective dielectric function is obtained using the Clausius-Mossotti equation (See Appendix A for details). The shell thickness, $L$, which is an "effective" monolayer-thickness in this model [181], was adjusted to $L=0.6 \mathrm{~nm}$ to reproduce the observed change in the relative intensity of the two peaks. The predicted spectra are in close agreement with the experimental results, barring the scaling factor of 5.3 discussed in Section 6.4.1. One may nevertheless notice that the theory predicts a larger blue shift at high concentrations than observed experimentally. This discrepancy is consistent with the expectation that the dye is preferentially adsorbed flat: as discussed in Figure 6.10 (b), the blueshift is related to side-by-side interactions between induced dipole perpendicular to the metal surface, and should disappear for flat adsorption. The general agreement between theory and experiment in Figure 6.12 (b) provides strong evidence that the observed 

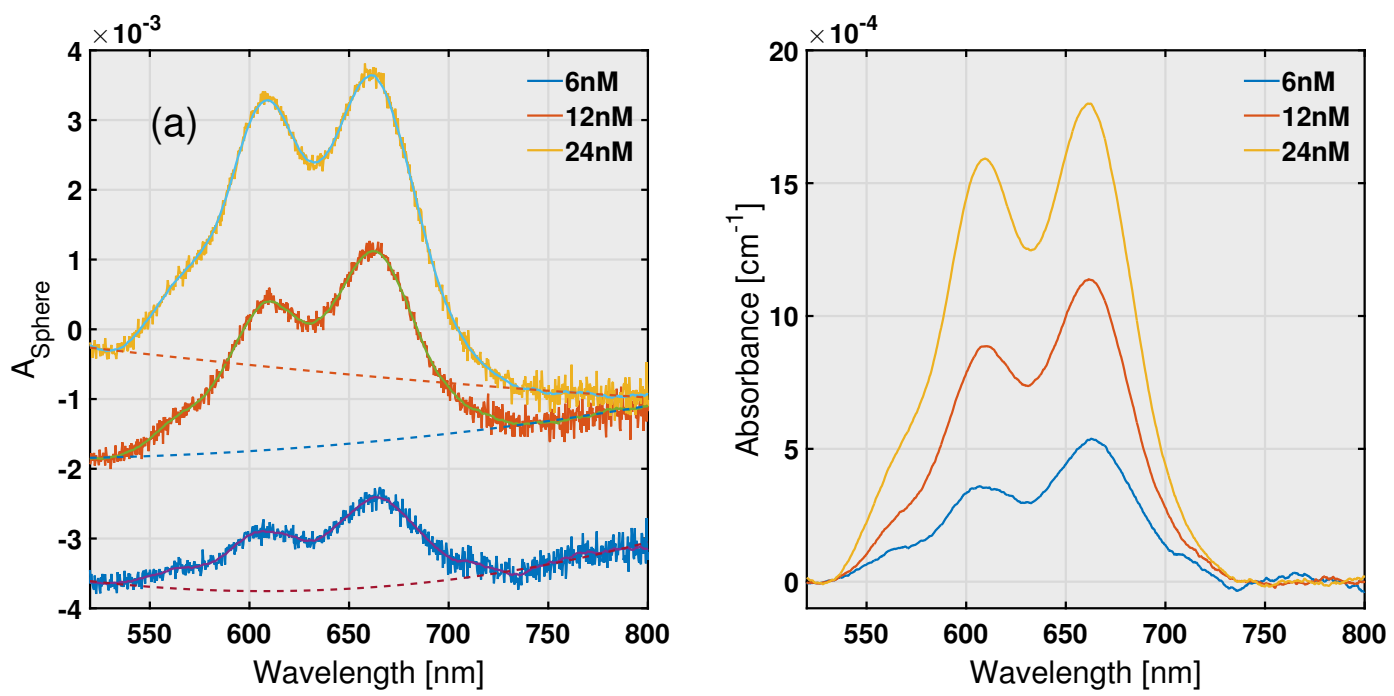

Figure 6.13: (a) Differential absorbance of Rhodamine 700 on Ag colloid (same data as Figure 6.12). The raw absorption spectra are first smoothed using a 31-pixel moving average filter. It is is clear from these plots that this step does not introduce any artifacts. Then, a second-order polynomial background (dashed lines) is subtracted from each spectrum to account for slowly-varying changes in the baseline between the sample and its reference. The resulting, path-length-corrected spectra, are shown in (b) as presented in Figure 6.12.

changes in relative peak intensities can be attributed to dye-dye interactions and that these effects can remain important even at very low concentrations, here down to $\sim 6 \mathrm{nM}$, in a regime where many surface-enhanced spectroscopy experiments are carried out.

\subsubsection{Post-processing and baselines}

Slow drifts in the lamp spectrum, slightly inaccurate referencing induced by minute variations in the colloid concentration, or small changes in sample geometry, can cause variations in measured intensity. This results in spectrally smooth backgrounds in the absorbance and differential absorbance measurements. To account for these instrumental limitations, standard baseline corrections are performed on all absorbance measurements. This is illustrated in Fig. 6.13, where the data post-processing that was applied to the experimental spectra presented in Figure 6.12 is shown for Rhodamine 700 at 6,12 and $24 \mathrm{nM}$ respectively. 

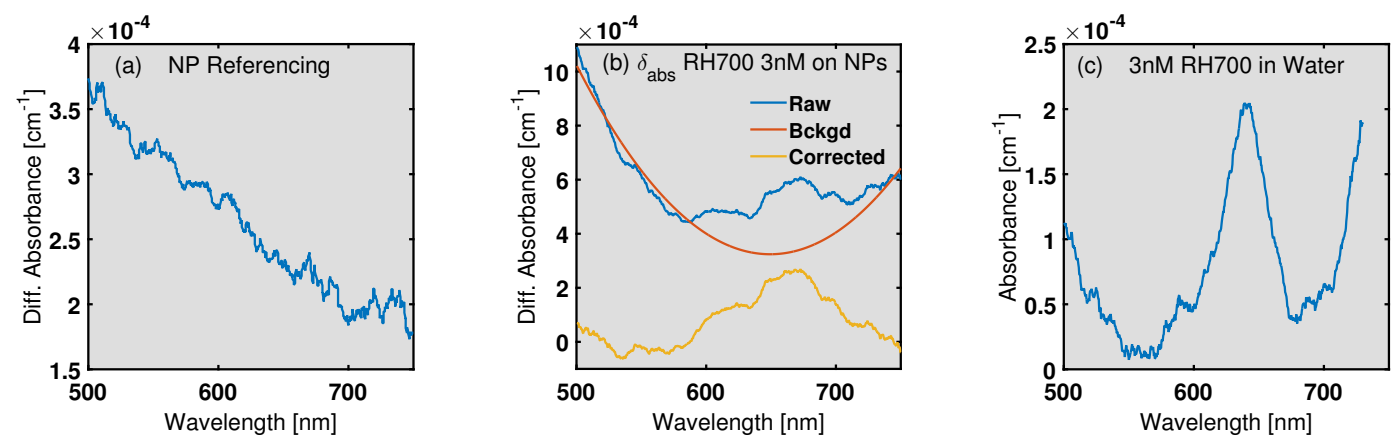

Figure 6.14: (a) Quantification of the error associated with replacing the $\mathrm{NP}+$ dye sample with the NP-only reference. This is here illustrated by measuring the absorbance spectrum of the silver colloid solution against itself (the solution is physically removed and replaced by an identical one). Spectral variations of the order of $10^{-4} \mathrm{~cm}^{-1}$ are typically observed. Although not observed on this example, spectrally-flat offsets (of up to $10^{-3} \mathrm{~cm}^{-1}$ ) are also commonly observed but easily removed. (b) Differential absorbance of Rhodamine 700 on NPs at a concentration $3 \mathrm{nM}$. The uncorrected spectrum is shown alongside a best-guess for the baseline and the corrected spectrum. It is clear that a large uncertainty remains at such low concentrations. (c) Absorbance spectrum of Rhodamine 700 in water at a concentration $3 \mathrm{nM}$.

\subsubsection{Low Concentration Approach}

The most direct way to determine if the low concentration dye regime has been reached, ensuring the measured spectrum represents the isolated dye molecule on the surface would be to measure a lower concentration than $6 \mathrm{nM}$ and compare the spectral shape of both measurements. If no spectral change is observed for the two concentrations, then one can safely assume that the regime has been reached where the dye concentration does not play a role in the measured spectrum. This is unfortunately extremely difficult to achieve because $6 \mathrm{nM}$ corresponds to an absorbance (in the sphere) of $\sim 5 \times 10^{-4}$. Attempting to measure absorbance signals below this becomes hampered by background issues, lamp drift and minute changes in colloid concentrations, all of which affect the quality of the obtained spectra. This is evidenced in Figure 6.14 (b) where $3 \mathrm{nM}$ of RH700 was measured. As is clear the background subtraction is problematic and thus the correct absorbance spectrum cannot be elucidated.

The baseline corrections in Figure 6.13 are only applicable if the baseline 
is sufficiently smooth and if the spectral variations are small compared to the sample spectrum to be measured. This ultimately limits the sensitivity of the CMSIS method. In the current implementation, the detection limit for the differential absorbance measurements is of the order of $\sim 10^{-4} \mathrm{~cm}^{-1}$, which arises from slight imperfections when referencing against the nanoparticle-only solution (Figure 6.14(a)). For a dye like Rhodamine 700 adsorbed on the nanoparticles, this corresponds to a concentration of $\sim 3 \mathrm{nM}$. As illustrated in Figure 6.14(b), the absorption spectrum can be observed, but the large uncertainties from the baseline corrections mean that the spectral shape cannot be extracted with sufficient accuracy to allow any reasonable analysis of the data. For comparison, also shown is an absolute absorbance measurement (referenced against water) for Rhodamine 700 in water at the same concentration of $3 \mathrm{nM}$ (Figure $6.14(\mathrm{c}))$, where referencing problems are also visible.

\subsubsection{Extracting the Modified Polarizability}

Two approaches can be used to deduce the modified Rhodamine 700 polarizability from its differential absorbance spectrum $\delta \sigma_{\text {abs }}(\lambda)$ measured at $6 \mathrm{nM}$. In the first one, an analytic model is chosen for the polarizability (a doubleVoigt-type peak as described in Appendix A) and the Mie-theory/effective shell model is used to predict the $6 \mathrm{nM}$ differential absorbance spectrum. The parameters of the polarizability are then adjusted to ensure agreement between predictions and experiments as the surface coverage is increased. This approach in principle works even if dye-dye interaction effects are important (providing they are captured accurately by the theory). This is the approach taken to reproduce the spectral evolution in Figure 6.12.

In the second approach, to avoid relying on the model correctly accounting for dye-dye interaction effects the modified absorbance can be directly deduced from from $\delta \sigma_{\text {abs }}(\lambda)$ corrected by the predicted plasmonic enhancement $\left\langle M_{\text {loc }}(\lambda)\right\rangle$, i.e. $\delta \sigma_{\text {abs }}(\lambda) /\left\langle M_{\text {loc }}(\lambda)\right\rangle$. The two approaches should be equivalent if dye-dye interaction effects are negligible, as dividing by $\left\langle M_{\text {loc }}(\lambda)\right\rangle$ does not take into account any dye-dye interactions. As shown in Fig 6.15 (plotted as molar absorbances, $\bar{\epsilon}(\lambda))$, the $6 \mathrm{nM}$ measurement corrected by $\left\langle M_{\mathrm{loc}}(\lambda)\right\rangle$ only differs from the analytic model for the polarizabilty by a minute shift of $1 \mathrm{~nm}$, therefore confirming the negligible influence of dye-dye interactions at $6 \mathrm{nM}$ 


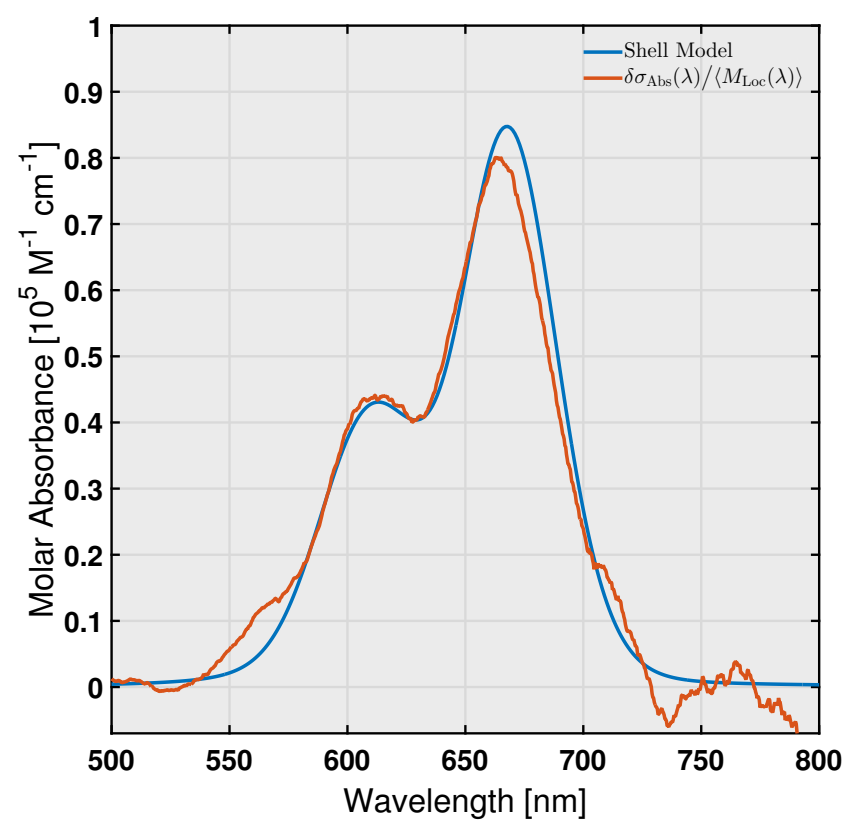

Figure 6.15: Modified molar absorbance of the dye RH700 deduced from the $6 \mathrm{nM}$ differential absorbance using two alternative approaches (see details in the text). The close agreement between the two spectra confirms the negligible influence of dye-dye interactions for concentrations below $6 \mathrm{nM}$.

for RH700, and reinforcing the validity of the derived modified polarizability. This also suggests that reducing further the dye concentration would result in the same spectrum, up to a negligible shift. To reinforce this point, correcting the higher concentration measurements by the plasmonic response would not result in a comparable spectrum to the predicted polarizability.

It is important to note that the measured absorbance (or polarizability) is in fact $\sim 5.3$ times less intense than that predicted by Mie-theory. This point is addressed later in Section 6.4.1.

\subsubsection{Extension to Other Dyes}

A natural extension to illustrating the success of the CMCIS is to investigate the surface absorbances of other dye molecules commonly used as reporters in surface enhanced techniques. As such, the study was extended to measure Rhodamine 6G, Nile Blue and Crystal Violet. The structure and properties of these dyes are discussed in Chapter 4 in detail. Importantly, between the four 
dyes used (RH700 included) the entire region between $500 \mathrm{~nm}$ and $700 \mathrm{~nm}$ can be investigated given the different resonance each dye exhibits, which could possibly highlight if the induced shifts in dye absorbance has any dependence on relative detuning from the plasmon resonance. There are a plethora of dye molecules available that have been used in many of the strong-coupling papers mentioned in Section 6.1.3 and studying their properties on the surface of nanoparticles would indeed be interesting, yet are beyond the scope of this thesis. The results obtained for the three other dyes studied are presented below.

\section{Rhodamine $6 \mathrm{G}$}

Rhodamine 6G (RH6G) in theory provides an ideal compliment to the RH700 results because it is from the same family of (xanthene) dyes as RH700 yet its main absorbance is in the 500 to $550 \mathrm{~nm}$ region, much closer to the main plasmon resonance at $430 \mathrm{~nm}$. This allows investigation of dye-nanoparticle effects across the visible region. Furthermore, RH6G has a slightly different chemical structure to RH700. As described previoulsy in Chapter 3, it posesses a xanthene ring and an extra carboxyphenol group which, from DFT calculations, is found to lie in a plane perpendicular to the main xanthene group [40]. The two main transitions of RH6G are observed at 530 and $350 \mathrm{~nm}$ in water and correspond to the S1 and S2 excitations. Reference [40] finds from TDDFT that the $\mathrm{S} 1$ dipole moment is oriented parallel to the xanthene ring as expected. The S1 transition is the one of interest for SERS, being the strongest and in the visible wavelength region where most SERS experiments are performed. A vibronic shoulder is also observed at $490 \mathrm{~nm}$ associated with the $\mathrm{S} 1$ transition. RH6G also does not contain the trifluoromethyl end group in RH700. Finally, given the widespread use of RH6G across spectroscopy, obtaining the surface modified absorbance on Ag nanoparticles is of huge interest to many fields.

The results for RH6G as measured in the CMCIS at increasing dye concentration are presented in Figure 6.16 (solid lines) along with the theoretical predictions (dashed lines) from Mie theory. The main feature of note is that the surface absorbance shows a red shift of between $14 \mathrm{~nm}$ and $17 \mathrm{~nm}$ from that in solution (See Appendix A for Voigt fits to the dye absorbance in water). The main $\mathrm{S} 0 \rightarrow \mathrm{S} 1$ resonance, normally at $527 \mathrm{~nm}$ in water is shifted to $544 \mathrm{~nm}$ 

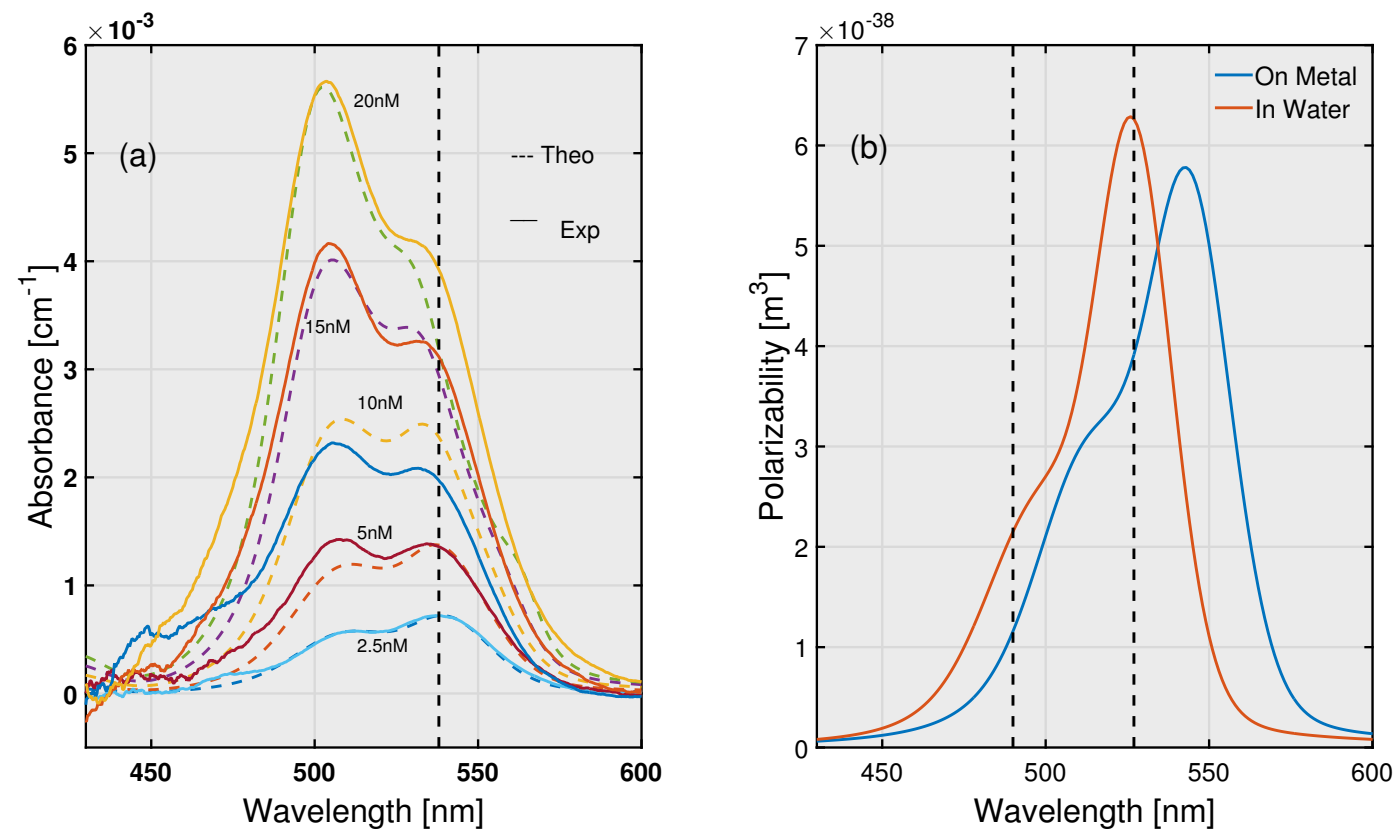

Figure 6.16: (a) Measured (solid lines) and predicted (dashed lines) differential absorbance spectra of Rhodamine 6G (RH6G) at concentrations of $2.5,5,10,15$ and $20 \mathrm{nM}$ on $30 \mathrm{~nm}$-radius $\mathrm{Ag}$ nanospheres $(8 \mathrm{pM})$. The dashed black line shows the blue shift of the peaks as the dye concentration increases. Note a scaling factor of $\sim 4.4$ is necessary to quantitatively match experiments and theory (see discussion in the text). The modified surface polarizability of RH6G was modelled as a double-Voigt-type resonance (see Appendix A), with the same oscillator strength as bare RH6G and parameters adjusted to fit the experimental surface absorbance spectrum measured at $2.5 \mathrm{nM}$. The effective thickness of the shell was adjusted to $0.2 \mathrm{~nm}$. (b) Comparison between the deduced intrinsic surface absorption spectrum of RH6G and the reference bare RH6G. 
while the $500 \mathrm{~nm}$ vibronic shoulder is now at $514 \mathrm{~nm}$. In a similar fashion to $\mathrm{RH700,} \mathrm{the} \mathrm{change} \mathrm{in} \mathrm{relative} \mathrm{peak} \mathrm{intensities} \mathrm{for} \mathrm{the} \mathrm{main} \mathrm{and} \mathrm{vibronic} \mathrm{peaks}$ occurs as the dye concentration increases, indicating the onset of dye-dye interactions. The onset of this interaction occurs at lower dye cocentrations that for RH700; for RH6G, even at $15 \mathrm{nM}$ the vibronic peak is more intense that the main transition, indicating that the dipole-dipole coupling for RH6G is stronger than for RH700. The reasons for this stronger coupling are difficult to pin down but is possibly linked to different adsorption geometries for both molecules (discussed later). Also of note is the shell thickness, $L$, in the Mie-theory model had to be adjusted to $0.2 \mathrm{~nm}$ to reproduce the earlier onset of dye-dye interaction. Again there is a slight discrepancy in the predicted versus measured spectral evolution but given the simplicity of the model used (neglecting the dye orientation), the agreement with experiment is remarkable.

RH6G, among other many other dyes, is known to readily form dimers in both solution at high concentrations and when adsorbed to particle surfaces $[182,183]$. It has been shown to adopt both the J-aggregate (blue-shifted) and $\mathrm{H}$-aggregate (red-shifted) on Ag surfaces [156]. The exciton description for the optical properties of molecular aggregates are shown in Figure 6.17 for illustration. In fact the aggregation characteristics of RH6G on Au nanoparticles have been studied in detail by $\mathrm{Hu}$ et al. [182] who attributed the appearance of a blue-shifted $507 \mathrm{~nm}$ and a red-shifted $537 \mathrm{~nm}$ band in the absorption spectra on the NPs to the formation of $\mathrm{H}$-aggregates and J-aggregates respectively, also known as herringbone aggregates. These results are reminiscent of the Mie-theory predictions in Figure 6.10. A number of points are worth noting in this study; firstly, the Rhodamine absoprtion peak closely matches that of the gold surface plasmon bands, which the authors note may be formed upon adsorption of RH6G. This causes problems, as mentioned before, in decoupling the plasmon and dye contributions to the spectra. Secondly, the dye concentrations used are large and aggregates will readily form on the NP surface. Finally, the authors claim that the blue-shifted peak is a result of J-type aggregates. This assumption, adopted also in other study [184] originates from Kerkers 1985 excellent review article, wherein the theoretical spectra of an Ag particle coated with Rhodamine B (not $6 \mathrm{G}$ ) exhibit blue shifts with respect to the bare dye. Hu et al. however neglect the fact that RH6G exhibits a vibronic shoulder at $496 \mathrm{~nm}$ in water and the results from the CMCIS exper- 
iments systematically show that a red shift occurs for both the main peak and the vibronic shoulder, appearing at $514 \mathrm{~nm}$ and $544 \mathrm{~nm}$ respectively. The parameters between the results presented herein and Reference [182] are quite different and makes direct comparison difficult. A natural extension to the results within would be to use gold nanoparticles to compare with the observations of Reference [182]. Again the advantage of the approach here is that ultra-low dye concentrations can be used and the true dye absorbance on the surface can be elucidated, and many of the more recent studies extended to other dyes interacting with nanoparticles $[184,185]$ all work at large dye concentrations and in regimes where the plasmon resonance interferes with the true dye resonance.

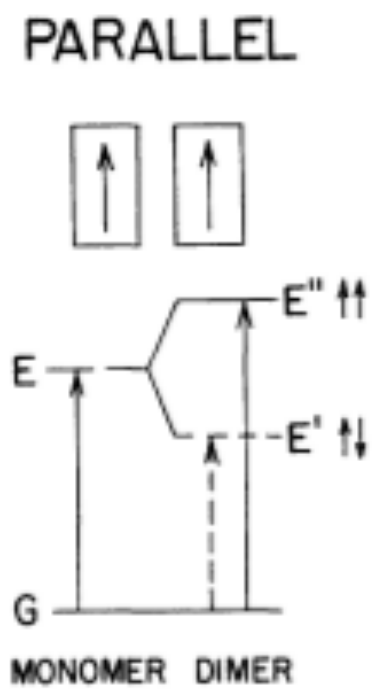

BLUE-SHIFT (Hypsochromic)
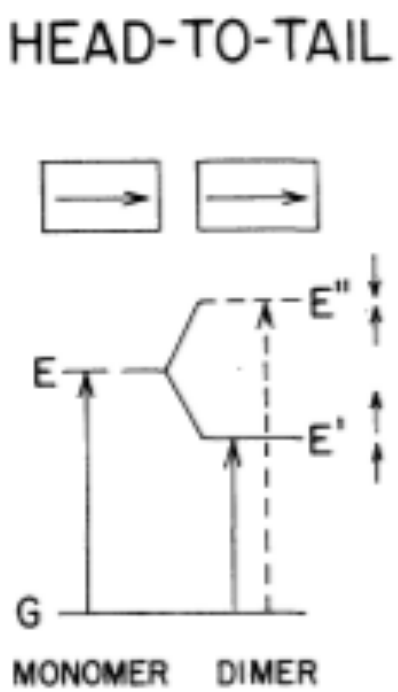

RED-SHIFT (Bathochromic)
OBLIQUE
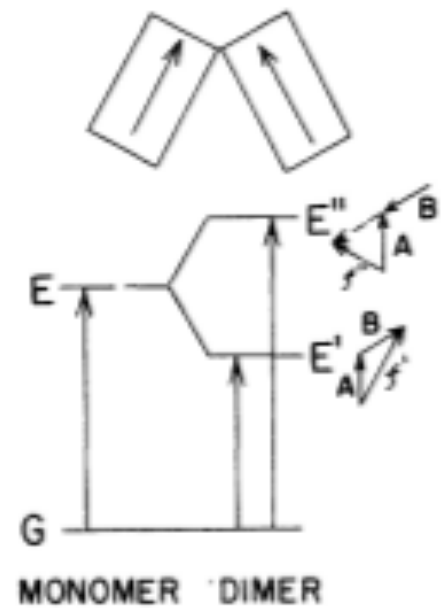

BAND-SPLITTING

Figure 6.17: Diagrams of exciton energy leves in molecular dimers with various geometrical arrangements of transition dipoles. Reproduced with permission from [176].

Despite the minor differences in spectral evolution, the results for RH700 and RH6G are strikingly similar and strengthen the interpretations of dye-dye coupling on the nanoparticle surface. With respect to the intrinsic shift of the dye resonances, given the molecular similarity between both dyes, the observed red-shift in both cases indicates the mechanism that modifies the absorbance must be similar. A qualitative interpretation here is that the orbitals of $\mathrm{Ag}$ atoms interact with the molecular orbitals of the molecules in a similar way, 
thus modifying the position of the HOMO-LUMO band for both dyes, akin to the Chemical Effect in SERS treatments [56]. The fact that the shift is bathochromic indicates the $\pi$-orbital electrons, responsible for the main dipole transitions in the visible, are less strongly bound to the nuclei of the aromatic backbone and are more easily polarised. Thus the electronic states of the molecules can be viewed as being "more de-localised" when adsorbed to the metal, requiring less energy (longer wavelength) for light absorption through dipole-transitions to occur. This interpretation would certainly need to be verified through time-dependent density functional theory (TDDFT) to model the combined molecule-nanoparticle excited states but such calculations are outside the scope of this thesis. Jensen et. al have performed such studies on RH6G coupled to Ag clusters [186] and found the main electronic resonance of the molecule can shift by $40 \mathrm{~nm}$ due to the presence of the silver cluster, showing qualitative agreement with the shifts observed here.

\section{Nile Blue}

Nile Blue (NB) has also been used extensively in SERS and its reduced quantum yield in water compared to other visible dyes allows easier investigation of resonance Raman cross sections as described in Section 6.1.2. To measure the absorption spectrum on nanoparticles would be of prime importance to resonant SERS studies and was in fact the original reason for beginning this study. For reasons addressed below however, it proves a difficult molecule to extract the surface absorbance of.

The results for NB as measured in the CMCIS are shown in Figure 6.18. As can be seen, the spectrum at $10 \mathrm{nM}$ is significantly broader and nosier than those of the Rhodamine dyes, exhibiting a maximum absorbance of only $~$ $4 \times 10^{-4} \mathrm{~cm}^{-1}$ in the 600 to $650 \mathrm{~nm}$ region. This value is approaching the current limit of detection of the CMCIS setup. As even at the same concentrations of RH700 and RH6G, Nile Blue poses a more difficult challenge to measure at low surface coverages. This is apparent for two reasons; firstly Nile Blue has an inherently lower molar extinction coefficient, $\bar{\epsilon}_{\mathrm{M}}$ of $\sim 6 \times 10^{4} \mathrm{~cm}^{-1} \mathrm{M}^{-1}$ than those of RH700 $\left(\sim 1 \times 10^{4} \mathrm{~cm}^{-1} \mathrm{M}^{-1}\right)$ and RH6G $\left(\sim 0.9 \times 10^{5} \mathrm{~cm}^{-1} \mathrm{M}^{-1}\right)$. This constitutes lower absolute signals to extract from the colloid background. Secondly, and as discussed later in Section 6.4.1, Nile Blue only shows an 
enhancement of $\sim 1.1$, the same order as RH700. While this is expected due to the dyes known flat adsorption geometry on Au surfaces [79] and SERS EFs of only 50 to 100 . As such, there is less benefit from local field enhancement (that is predominantly perpendicular to the Ag surface) when adsorbed to the NP surface to allow the absorbance signal to be extracted.

Despite these complications, it is still clear from the measured spectrum at $10 \mathrm{nM}$ that Nile Blue does not show significant shifts in peak position but does show a noticeable broadening across the 500 to $700 \mathrm{~nm}$ region. Interpretations based on these observations are that Nile Blue has no very little chemical interaction with the silver surface and is only physisorbed through electrostatic interactions, and thus the perturbation to the electronic states is minimal. The flat adsorption geometry is intuitive from an energy-minimisation point of view whereby a planar molecule is more-likely to lie flat on a surface where no specific chemical bond is prevalent.

The low intensity and broadened spectra observed for Nile Blue make concentration dependent studies difficult to perform and any attempt at reproducing the observed spectral changes with increasing dye concentration was unsuccessful due to problematic backgrounds. Nile Blue also induces aggregation more readily that the other dyes invalidating the differential absorbance technique. Further improvements for the CMCIS setup would be required to increase the sphere $L$ factor so that lower concentrations of Nile Blue can be more readily measured, along with a better understanding of the effect of NB on the stability of the colloids. Nevertheless the results show that the nature of modified and enhanced dye spectrum is specific to the dye being studied and depends on its interaction with the nanoparticle surface.

\section{Crystal Violet}

Of the four dyes measured, CV exhibits the most dramatic change in absorption, as shown in Figure 6.19, with a $\sim 90 \mathrm{~nm}$ blue shift to $500 \mathrm{~nm}$ from its main absorption peak in water at $590 \mathrm{~nm}$ and second red-shifted peak at $\sim 600 \mathrm{~nm}$. Such a dramatic spectral shift suggests a major chemical change for the adsorbed dye. The CV absorption spectrum can change dramatically in varying pHs [187], where the level of protonation of nitrogen groups directly affect 


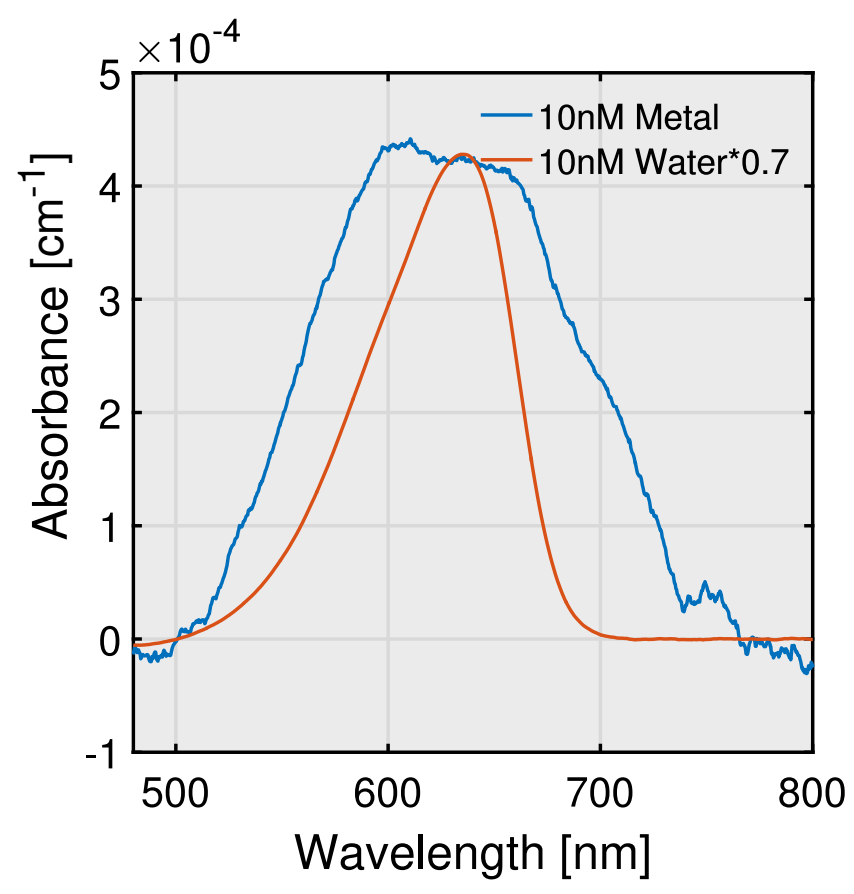

Figure 6.18: Surface absorption spectrum (blue) of Nile Blue on $60 \mathrm{~nm} \mathrm{Ag}$ colloids at $10 \mathrm{nM}$ as measured in the CMCIS compared with the same concentration measured in water (red).

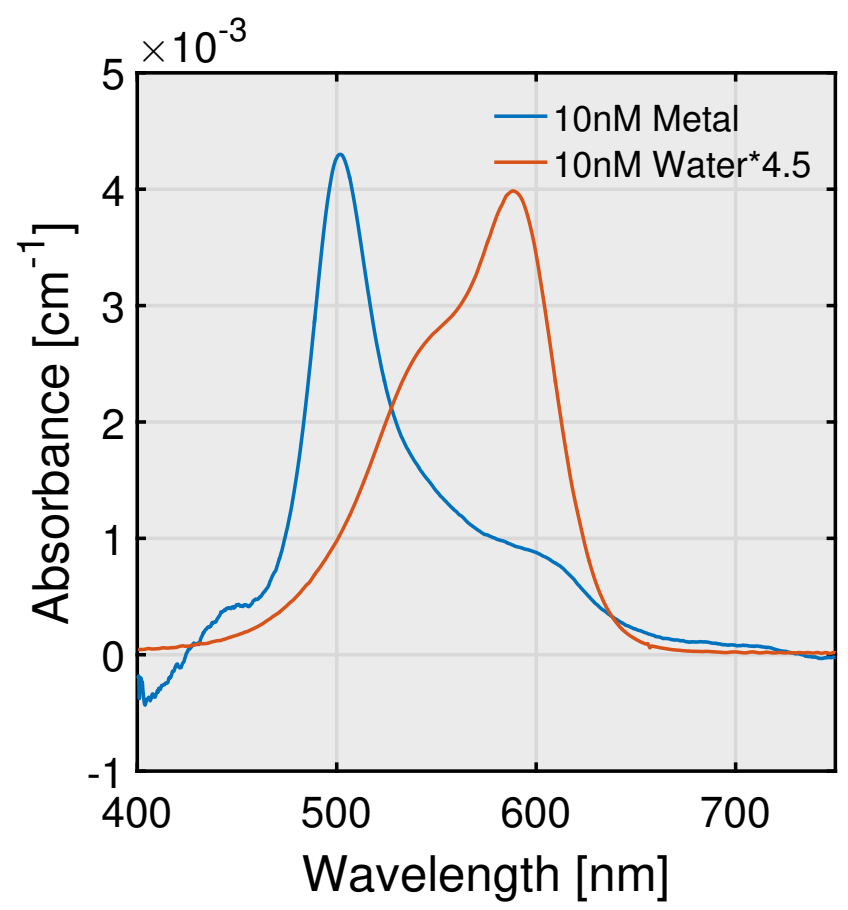

Figure 6.19: Surface absorption spectrum (blue) of Crystal Violet on $60 \mathrm{~nm}$ $\mathrm{Ag}$ colloids at $10 \mathrm{nM}$ as measured in the CMCIS compared with the same concentration measured in water (red). 
the resonance wavelength of the main absorption peak. It is speculated that chemical binding to silver may have a similar effect here, but the full mechanisms behind this shift are not fully elucidated. The electronic structure of $\mathrm{CV}$ and the origin of its absorption spectrum, particularly the higher energy shoulder at $\sim 550 \mathrm{~nm}$, has been the subject of intense debate and investigation for many years [188]. Much of this debate is based around whether the vibronic shoulder arises from two degenerate ground states or excited states, due to symmetry lowering from the $D_{3}$ configuration, solvation effects or the presence of isomers in solution $[187,188]$. The discussion around this is long and detailed, and a recent work [189] uses hyper-Raman studies to propose the shoulder arises from the existence of two excited states, where the degeneracy is lifted when dissolved in a polar solvent [190]. These obscure nature of the electronic states of $\mathrm{CV}$ could be the reason for its markedly different absorption spectrum on silver, whereby the degeneracy is lifted upon adsorption. While these arguments are merely qualitative, the observed spectrum constitutes one of the first direct, experimental indications of a strong chemical interaction with a nanoparticle surface, without the need for charge-transfer interpretations based on SERS spectra. The implications for this shift is discussed later in Section 6.4.2. Again, the TDDFT calculations of Jensen et al. [186] have shown a red-shift in absorption of $40 \mathrm{~nm}$ for CV coupled to $\mathrm{Ag}$ clusters; clearly the experimental results here can be used as a new benchmark to improve such models and elucidate the nature of the chemical interactions between the molecule and the metal.

\subsection{Agreement with Theory}

\subsubsection{Enhanced Absorbance}

Up to this point, only the spectral shifts in dye absorbance induced by adsorption the the NP surface has been discussed, neglecting any mention of the absorption enhancement, which is arguably as important as any spectral shifts. To obtain the magnitude of the absorption enhancement for dyes adsorbed to the NPs with that in water, it is best to consider oscillator strengths rather than simply comparing relative peak intensities. This approach ensures 
that broadening and changes in spectral shape can be accounted for when comparing with the absorbance spectrum in water.

From the classical treatment of a simple-harmonic oscillator, the oscillator strength, $f$ can be deduced from the UV-Vis absorption spectrum by [55]

$$
f=\frac{4.32 \times 10^{-9}}{n_{M}} \int \bar{\epsilon}(\bar{\nu}) d \bar{\nu},
$$

where $\bar{\nu}=1 / \lambda$ is the wavenumber in $\mathrm{cm}^{-1}$ and $\bar{\epsilon}(\bar{\nu})$ is the decadic molar absorption coefficient in $\mathrm{cm}^{-1} \mathrm{M}^{-1}$. In practice, the measured absorption spectrum on the NPs are first corrected for the sphere response as normal and converted to molar absorbance by normalization to dye concentration. From here the enhancement in absorbance is simply calculated from Equation 6.9 by the ratio of oscillator strength on the NP surface to that measured in water. Figure 6.20 shows the results of this calculation for all four dyes RH700, RH6G, CV and Nile Blue.

From the calculated oscillator strengths, the enhancements are $\sim 1.2$ for RH700 and NB, while RH6G and CV exhibit larger ehnahcements of the order of 4.1 , the largest being $\sim 4.4$ for RH6G. These values seem particularly low when compared to the predicted local fields from Mie-thoery but can be explained due to non-ideal adsoprtion geometries.

As mentioned in Section 6.3.3.3, the oscillator strength for the measured absorbance for RH700 at $6 \mathrm{nM}$ is $\sim 5.3$ times smaller than that of the predicted oscillator strength from Mie-Thoery. While this possibility cannot be excluded, it is more likely that the oscillator strength is unchanged and that the magnitude of the actual absorption enhancement is smaller than predicted (by the same factor $\sim 5.3$ ). In fact, electromagnetic theory predicts that for a $30 \mathrm{~nm}$-radius Ag sphere, the average absorption should be enhanced by a factor of $\left\langle M_{\text {Loc }}\right\rangle=6.4$ at $664 \mathrm{~nm}$ but this implicitly assumes an isotropic polarizable dipole. Most dyes however (including RH700) have a strongly uniaxial transition dipole moment along the $\pi$-conjugated backbone of the molecule. Because the local electric field is primarily perpendicular to the metal surface (with only $\left\langle M_{\mathrm{loc}}^{\|}\right\rangle=0.17$ for the parallel component at $664 \mathrm{~nm}$ ), the observed enhancement in absorption will be strongly dependent on the preferential orientation 


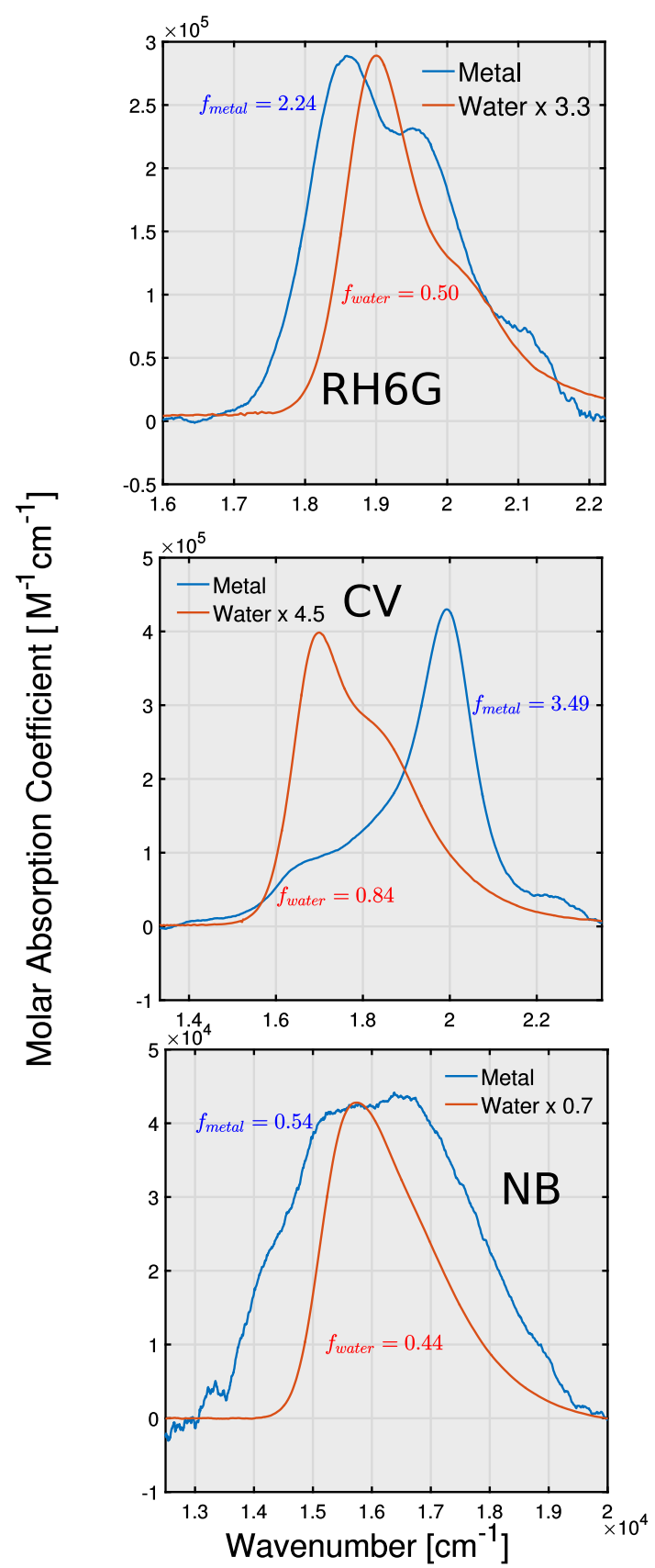

Figure 6.20: Molar absorption coefficients in water (red) and on colloids (blue) for (top) RH6G, (middle) CV and (bottom) Nile Blue, plotted in wavenumbers. The oscillator strengths of each transition as calculated by Equation 6.9 are shown in each figure from which the enhanced absorbance can be calculated. 
of the adsorbed dye on the NP surface. The observed reduced absorption enhancement for RH700 can therefore be attributed to an adsorption orientation preferentially flat (or at a small angle) on the surface, which is expected for dyes with such a planar structure. Similar effects are seen for Nile Blue, which also shows an enhancement of $\sim 1.2$. These observations are consistent with previous results for Nile Blue adsorbed to Au surfaces [82], where careful polarization dependent studies of the SERS signals showed that Nile Blue has a flat adsorption properties. Furthermore, SERS measurements of Nile Blue on Ag colloids (not shown), when correctly compared to the bare Resonance Raman cross sections from Reference [81], consistently show enhancement factors of the order of 50 only, confirming that flat adsorption geometries do can not benefit from the strongly perpendicular local electric field on the NP surface.

In contrast to RH700 and Nile Blue, both CV and RH6G show modest enhancements of 4 to 4.5 . While these values again seem low when one considers the much larger Raman enhancements often quoted for these molecules (from Chapter 4 typical AEFs for R6G are of the order of $10^{4}$ ), they are in fact only $\sim 3$ times less than the predicted values from Mie-theory as shown previoulsy in Figure 6.7, where the isotropic enhancement is $\left\langle M_{\text {Loc }}\right\rangle$ is $\sim 14$ at $540 \mathrm{~nm}$. While the adsorption geometry of CV is difficult to infer due to the significant shift in its surface spectrum along with the high symmetry of its structure, the RH6G results suggest the molecule adsorbs with it's electronic transition dipole at some angle $90^{\circ}<\theta<0^{0}$ with respect to the NP surface. This interpretation is consistent with RH6Gs non-planar geometry compared (the carboxyphenol group oriented perpendicular to the main backbone) to RH700, allowing it to adopt a more perpendicular orientation on the surface and thus benefit from the larger perpendicular local electric field. Further supporting this observation is the fact that RH6G and CV consistently show Raman Enhancements much larger than that of Nile Blue which is indicative of a dipole transition oriented preferentially to the perpendicular component of the local field. Probing the molecular orientation on a surface is extremely difficult to achieve, and recent experimental work by Matthies et al. [191] has shown that the orientation of DTTC dye moleclules adsorbed to gold surfaces can be eludicated only through careful angle-resolved UV-Vis measurements, where extreme care is taken to characterise the substrate and model the optical properties of the system. Interestingly, the authors note that using an 
isotropic, randomly oriented dye layer fails to reproduce the measured spectra as a function of incident light angle. Only when the dye-layer anisotropy is incorporated (with the main dipole axis oriented parallel to the surface) does the model accurately reproduce the results.

It is clear from the discrepancy between theory and experiment that while the Mie-theory shell accurately reproduces the spectral changes with increasing dye coverages, is fails to correctly predict the magnitude of the enhanced absorbance. This is attributed to the assumption that the dielectric function of the dye layer in the shell model is isotropic. In reality, for fixed adsorption geometries, an anisotropic epsilon would need to be included and by varying the adsoprtion angle with respect to the local field, the predicted enhancement would be reduced to match the experiment accordingly.

\subsubsection{Modified Absorbance in SERRS}

As discussed early on in this chapter, a natural consequence of a modification of the absorption spectrum of dye molecules is the effect this will have on measuring SERS cross sections of such resonant molecules. A dramatic modification of the molecular electronic resonances would have direct implications on the wavelength dependence of measured SE(Resonant)RS enhancement factors. To investigate this, the SERRS EFs of Crystal Violet, whose absorption spectrum shows the most significant modification from the bare molecule in water, was measured across the visible region using a number of different laser excitations.

Crucial to extracting the true SERRS Raman Excitation Profile (REP) of a resonant molecule is to correct the measured EFs for the plasmonic dispersion of the SERS substrate (here the $60 \mathrm{~nm}$ Nanocomposix Ag colloids). It is well understood [16] that the SERS EF will vary as a function of excitation wavelength used depending on its position with respect to the main dipolar resonance of the substrate. For example, for a $30 \mathrm{~nm} \mathrm{Ag} \mathrm{sphere} \mathrm{the} \mathrm{expected}$ average SERS EF, $\langle F\rangle$ is predicted from Mie-theory to be $\sim 1300$ while at $633 \mathrm{~nm}$ it is only about 100. As such, measuring the wavelength dependence of the SERS EF of a molecule will by definition be affected by this underlying plasmon dispersion and will conceal the true strength of the Raman cross 
section of the molecule on the metal surface. To account for this effect, a nonresonant but Raman-active molecule, bi-pyridine-ethylene (BPE) was used as a reference molecule to account for the wavelength dependence of the colloid's enhancement factors. Because BPE is resonant in the UV region, any spectral dependence of its enhancement factor as measured on the colloids will reveal the plasmonic dispersion, which can then used to correct the CV enhancements. This method has scarcely been used in the past and is worth noting is neglected in the REPs measured by Lombardi et al. in Reference [139]. BPE also readily adsorbs to silver colloids and has been used previously in SERS measurements [131].

\section{Experimental Setup - T6400}

Because the measurement of REPs require a range of laser lines, the Raman setup used in these measurements was different than those used in Chapter 4. An Argon-Krypton laser with variable excitation from $458 \mathrm{~nm}$ to $647 \mathrm{~nm}$ was used in conjunction with a Jobin-Yvon T6400 triple subtractive spectrometer to acquire spectra. Raman scattered light was collected in the $90^{\circ}$ scattering configuration as described in [81]. A $75 \mathrm{~mm}$ collection lens was used to focus the scattered light onto the spectrometer entrance slits, that were set to $200 \mu \mathrm{m}$. A 600 lines per mm grating was used to collect spectra from 400 to $1800 \mathrm{~cm}^{-1}$. Samples were held in $10 \mathrm{~mm}$ quartz cuvettes, placed perpendicular to the incident beam. To avoid reabsorption of the scattered Raman photons, the laser was focused as close to the cuvette wall as possible.

Each wavelength measurement consisted of first aligning the collection optics such that a sharp image of the laser passing through a cuvette of colloids was obtained on the spectrometer camera. Slight changes in beam path when changing laser line causes the alignment to be altered. The spectrometer was then calibrated using the $516 \mathrm{~cm}^{-1}$ line of 2B2MP. Average SERS spectra of $10 \mathrm{nM} \mathrm{CV}$ and $100 \mathrm{nM}$ BPE in Nanocomposix colloids (premixed with $1 \mathrm{mM}$ $\mathrm{KCl}$ ) were then measured at each wavelength. Integration times were adjusted such that good signal to noise ratios were achieved. For the calculations of enhancement factors, 2B2MP was also measured at each wavelength under the same conditions, removing any wavelength dependence of the spectrometer response. Enhancement factors were then calculated in the standard fashion 
as outlined in Chapter 3, by comparing CV and BPE peak intensities to the $516 \mathrm{~cm}^{-1}$ mode of $2 \mathrm{~B} 2 \mathrm{MP}$ and correcting for concentration and bare Raman cross section of the analyte. The bare Raman cross sections for CV were taken from those measured in Reference [79] while BPE cross sections were measured at $1 \mathrm{mM}$ in ethanol and compared to the 2B2MP cross section at each wavelength. Because of the low Raman cross section of BPE and the long integration times required in SERS measurements, a water spectrum at each wavelength was also taken and subtracted from the BPE spectrum to remove problematic backgrounds.

\section{SERS Excitation Profiles}

Results of the wavelength dependent SERRS measurements of CV on Nanocomposix colloids are presented in Figure 6.21. Typical background corrected SERS spectra for BPE and CV are shown in (a) where the excitation wavelength was $532 \mathrm{~nm}$ and the AEFs are indicated for the $1605 \mathrm{~cm}^{-1}$ mode of $\mathrm{BPE}$ and the $1620 \mathrm{~cm}^{-1}$ mode of CV. In (b), the wavelength dependent AEFs of BPE (red triangles) and CV (blue stars) are plotted. The CV excitation profile shows the strongest response at $458 \mathrm{~nm}$ and a minimum response at $568 \mathrm{~nm}$, increasing again at $633 \mathrm{~nm}$ and $647 \mathrm{~nm}$. As mentioned above, any spectral dependence of enhancement factors for a resonant molecule are a convolution of both the molecules resonant (absorption) profile and the underlying plasmonic dispersion of the substrate. The plasmonic response is clear in the excitation profile of BPE, whose enhancement factor is here normalised correctly for the standard non-resonant $\omega^{4}$ dependence [56] of Raman cross section on excitation wavelength. Thus the BPE SERS profile represents the wavelength dependence of the SERS EF for the colloids used here. The BPE profile (scaled by a factor of 10 for comparison) in Figure 6.21 shows a maximum enhancement at $532 \mathrm{~nm}$ and gradually decreases as the excitation moves to the red. While a $60 \mathrm{~nm}$ silver sphere should show a maximum SERS EF in the $430 \mathrm{~nm}$ region, this trend may be due to the presence of either a small amount of aggregates in solution that do not show up in UV-Vis spectra of the colloids or the non-spherical shape of the particles that is not accounted for in Mie-theory. Nevertheless, the BPE profile can be used to correct the CV profile by normalizing the CV AEFs to the BPE EFs at each wavelength, 

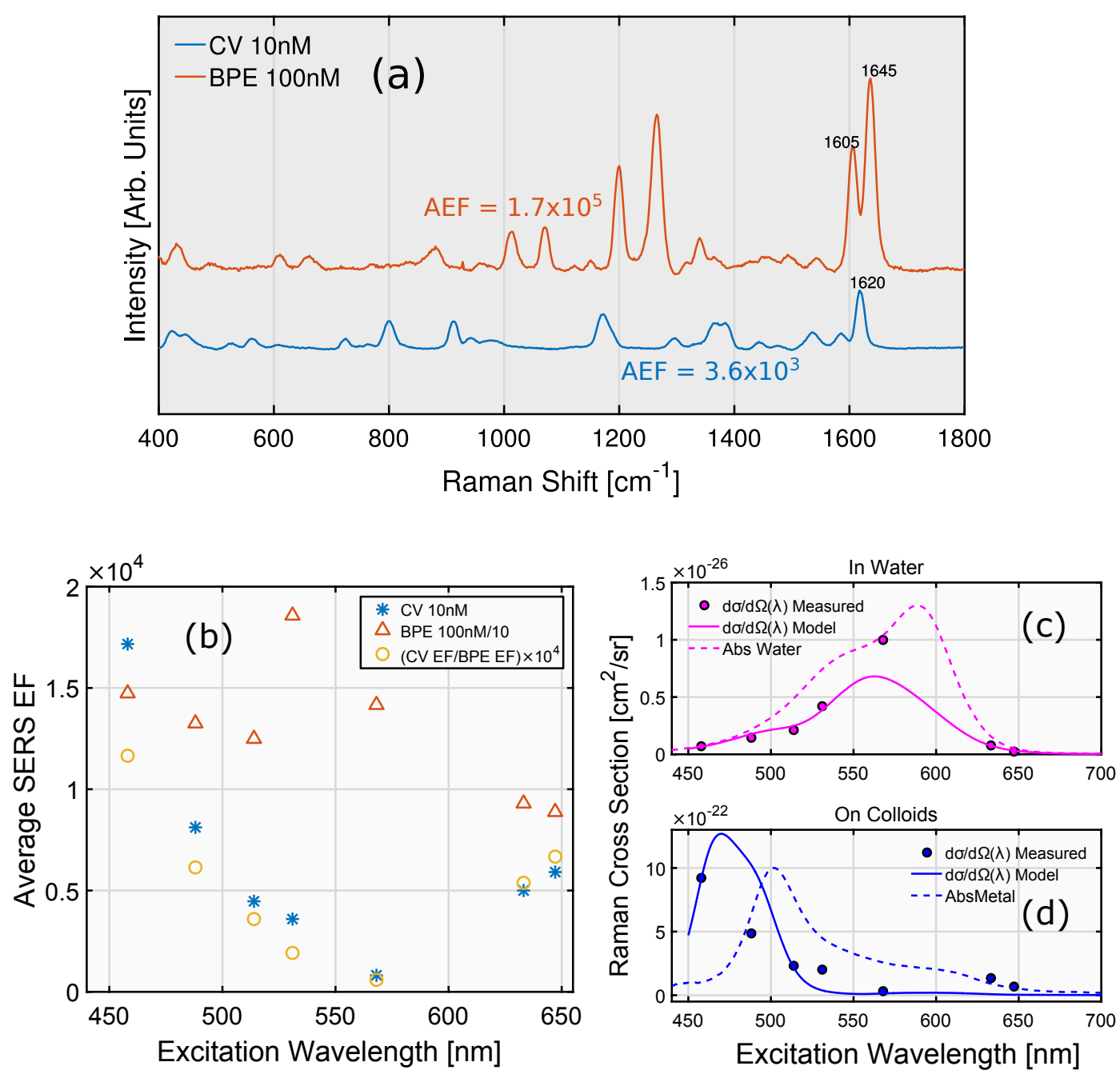

Figure 6.21: (a) Average SERS spectra of CV (10nM) and BPE (100nM) measured on Nanocomposix colloids in $1 \mathrm{mM} \mathrm{KCl}$ at $532 \mathrm{~nm}$ laser excitation. (b) Wavelength dependent average enhancement factors of the $1620 \mathrm{~cm}^{-1}$ peak of CV and the $1605 \mathrm{~cm}^{-1}$ peak of BPE (scaled by a factor of 10), along with the $\mathrm{CV}$ enhancements corrected by the BPE enhancements, removing the plasmon dispersion in the CV enhancements. (c) Measured (pink dots, from Ref. [79] and predicted (solid pink line) Raman Excitation Profile of the $1620 \mathrm{~cm}^{-1}$ mode of $\mathrm{CV}$ in water. The dashed line is the absoprtion spectrum of $\mathrm{CV}$ in water that was used in the optical transform model. (d) Measured (blue) and predicted (solid blue line) SERS REP of the $1620 \mathrm{~cm}^{-1}$ mode of CV adsorbed to Ag colloids. The modified absorption spectrum of CV on the colloids is shown (dashed line) that was used in the optical transform model. 
removing any spectral dependence of enhancement factor. The corrected CV EFs are shown as orange circles in (b).

Using the BPE correction method, the wavelength dependence of the CV cross section on the colloids is plotted in Figure 6.21 (d), along with the modified absorption spectrum measured in the CMCIS setup (blue shaded plot). The corresponding REP and absorption spectrum in water also plotted in (c) for comparison, where the measured CV cross sections were taken from Reference [79]. It is clear from the SERS measurements in (d) that the CV cross section exhibits a significantly different REP when adsorbed to the metal than in water. In water, the Raman cross section of $\mathrm{CV}$ is strongest at $568 \mathrm{~nm}$ and the REP tracks the absorption spectrum in water strongly as expected. In contrast, on colloids the REP tracks more closely the modified absorption spectrum measured in the CMCIS setup. Interestingly the cross section shows a maximum at $458 \mathrm{~nm}$, blue-shifted with respect to the absorption maximum at $\sim 500 \mathrm{~nm}$. The SERS REP then tracks the modified absorbance in a similar fashion to that measured in water, decreasing in intensity as the laser wavelength is increased. The REP does show an interesting trend in that there is an increase in cross-section at $633 \mathrm{~nm}$ and $647 \mathrm{~nm}$. The reasons for this are unclear at this point but it is possible that there is a second resonance still present for $\mathrm{CV}$ on the surface, as evident from the shoulder visible at $\sim 610 \mathrm{~nm}$ in the absorption spectrum thus recovering some of the resonance contribution to the Raman cross section at these wavelengths.

Finally the solid lines in Figure 6.21 (c) and (d) represent the predicted REPs based on the measured absorption spectrum, computed from the optical transform model given by Equation 6.7 introduced earlier. In (c), the REP is computed using the absorption spectrum of $\mathrm{CV}$ in water, while in $(\mathrm{d})$ the model is applied to the modified absorption data for CV measured in the CMCIS. The computed REP is fit to the experimentally measured SERS data by changing the Franck-Condon scaling factor in Equation 6.7. While the predicted REP in (a) is in excellent agreement with the measured profile, the model reasonably reproduces the general trend of the measured REP on the metal. There are a number of discrepancies, mainly in the underestimation of the $633 \mathrm{~nm}$ and $647 \mathrm{~nm}$ cross-sections but overall the fit is surprisingly good. There may a number of factors not captured by the standard optical transform model (some of the approximations may no longer be valid), but by simply 
using the measured absorption profile of the molecule on the metal, the general SERS REP can be reproduced; a maximum cross-section is predicted between 450 and $470 \mathrm{~nm}$, blue shifted with respect to the main electronic resonance. Similar trends are observed for the molecule in water (Figure 6.21 (c)). Further investigation of how the optical transform model may be extended to match better the SERS REPs will be needed but the initial results here are extremely promising for predicting the response of resonant molecules on metallic surfaces where chemical effects are important. Apart from the intensity borrowing approach used by Lombardi et. al [139], the application of the optical transform model to SERS spectra has not been investigated previously.

\subsection{Conclusion}

The proposed technique presented, based on the measurement of the differential absorbance of a sample inside an integrating sphere, provides a unique approach to experimentally access the surface absorbance spectra of chromophores adsorbed on NPs. It could also be directly applied to the study of non-metallic nanoparticles, and using a UV light source, to molecules other than dyes.

The results clearly highlight the great potential of this technique for ultrasensitive absorbance measurements in the presence of strongly scattering media, such as solutions of metallic colloids. The ability to measure the surface absorbance of dyes on metallic NPs paves the way for more detailed studies of both the dye-surface and dye-dye interactions. Recent related works in this area $[191,192]$ have been limited to adsorbed molecules on flat metal films and exposed to air, using polarization- and/or angle-dependent transmittance/reflectance spectroscopy. In such studies, it is extremely challenging to reliably control the molecular surface coverage/concentration because of the solution-to-surface transfer step. Moreover, the ambient medium can have a strong effect on the absorbance of dyes and the results obtained in air cannot be easily compared to the reference measured in water. The CMCIS method mitigates both of these issues by working in solution and can reliably reach sub-monolayer concentrations. 
Two important conclusions stand out from this study, which are expected to have many implications. Firstly, modifications of the intrinsic dye polarizability upon adsorption on metallic NPs appear to be the norm rather than the exception, and secondly, further modifications arising from dye-dye interactions remain important even at relatively low surface coverage, in fact in a range relevant to many experiments. Both of these effects should be carefully considered for many experiments involving dyes adsorbed on the surface of metallic NPs, notably molecular plasmonics (strong coupling, plexcitonics) and SERS, SEF, and other surface-enhanced spectroscopies. As an example, the experimental determination of SERS enhancement factors [20] typically assumes that the polarizability of the probe molecule is not modified upon adsorption. In view of the results, this assumption should clearly be revisited for experiments in resonance or pre-resonance conditions, with implications for the debate over the "chemical enhancement" mechanism [37]. While a small shift as observed for RH6G may not have any significant effect, the consequences of the large shift observed for CV should be major, and for example be clearly evident in wavelength-dependent measurements of SERS enhancement factors as shown in Section 6.4.2. By the same token, resonance shifts like those observed for RH700 and RH6G would result in weaker enhancement factors when measured at the resonance of the bare dye, which may explain why SERRS enhancement factors are often smaller than expected from theory [193]. Similarly, in the context of molecular plasmonics, most studies are interpreted in terms of coupling between dye and plasmon resonances, where the dye (or $J$ aggregate) spectrum is assumed to be unchanged in contact with the metal. While this may be the case for some molecules, the results suggest that such an assumption should be carefully checked on a case-by-case basis, and that dye-dye interactions and/or absorbance modification may play a major role in interpreting experimental results.

Beyond those direct consequences, it is anticipated that the experimental findings will spark a fruitful discussion on the interpretation of the observed spectral changes for different molecules. New experimental access to such a regime of molecule-metal interaction with a non-obtrusive optical technique will undoubtedly entice further tests and refinements of DFT predictions of the resonance of dye-metal complexes. It will also offer a deeper insight into the subtle interplay between electromagnetic effects, including selection rules 
linked to preferential orientation on the surface, and those of a more chemical nature attributed to changes in the electronic configuration of a molecule physisorbed or chemisorbed onto a surface. A refined understanding of these mechanisms will have a profound impact in the interpretation and further development of surface-enhanced Raman scattering, chiroptical, or fluorescence spectroscopies. 


\section{Chapter 7}

\section{Conclusion}

The work in this thesis represents two major steps towards improving both the understanding and reliability of SERS as an analytical technique; the work in Chapter 4 establishes a new protocol that drastically improves the reproducibility of SERS in colloidal solutions, while Chapter 6 represents the first direct link between the modified absorbance of dyes on metallic colloids and a chemical effect in SER(R)S. These two points are expanded on below:

- The role of the dilution of analytes on the SERS EF in colloidal solutions has been elucidated, and has been shown to have a crucial effect on analyte distribution across the solution. This new insight highlights vividly that the half-half dilution method should be adopted as standard dilution procedure in colloidal solutions to avoid sample non-uniformities and mitigate irreproducibility in estimating SERS EFs, both for average and single-molecule measurements.

- The ability to routinely measure the modified and enhanced absorbance of analytes on metallic surfaces has been established. It has been shown that, through the measurment of four common SERS analytes, modifications of the absorption spectrum of dye molecules upon adsorption to metallic colloids are the norm rather than the exception, and this surface absorbance can be systematically measured at sub-monolayer coverages using the new methodology. This new tool allows direct insight into chemical effects in SERS, using the example of the relationship between the modified absorbance of CV and its SERRS Raman Excitation Profile. 
It must be said that when this thesis was started in the middle of 2012, SERS was an already mature technique and many of the initial problems associated with reproducibility had been put to bed. Probably the key ingredient in this very early development was the establishment of a standardised definition of the SERS EFs [12] and the methodology required to estimate these values. This constituted a major step forward in allowing comparison of SERS results from different studies, performed in different labs around the world. Before then, the field was hampered by varying definitions and protocols of preference which only serve to hinder the development of the (any) technique. With the adoption of the rigorous definitions outlined in [12], the reliability and routine estimation of EFs has dramatically improved, to the point where it is common procedure in the literature to explicity indicate how SERS EFs were calculated. From this perspective, the results of Chapter 4 can be seen in the same light; prior to the investigation of the effects of diffusion/adsorption on analyte distribution, there was no standardised protocol in the literature for the dilution of SERS samples in colloids. While the effect of cationic dyes on the aggregation of colloids has been studied in the past [95,96], and correlations between SERS intensities and molecular coverages have been observed [99], the link with dilution procedures had not been investigated ${ }^{1}$. Given the demonstration in Chapter 4 that using large dilution factors introduces a wealth of undesirable problems for quantitative estimation of SERS EFs, it is reasonable to say that the half-half dilution method should, and could, be adopted in the same fashion as the protocols outlined for EF determination. Without such a standard protocol, or at the very least specification of how exactly analytes were diluted, comparison between studies is made very difficult. As such, the results in Chapter 4 represent a step forward in further standardising SERS experiments across the field.

While the dilution study was performed on the most general SERS substrate, citrate-reduced Ag colloids, with two common SERS probes, a natural extension would be to investigate the extent of the problem for other types of colloidal systems. The first of these could be to apply the same steps in solutions of gold colloids, which are quickly becoming preferable to silver colloids as substrates for analytical applications due to their improved dispersity and

\footnotetext{
${ }^{1}$ At the time of publication of [92] , an article was published during the submission process that found that by using large dilution factors of Malachite Green, clusters of colloids could be induced [194].
} 
lower reactivity with the surrounding environment. The potentially different chemical interaction between the gold surface and dye molecules may offset the distribution of analytes and it would be interesting to investigate this. A second point of interest would be to investigate the effect of colloid surface species on the extent of the dilution problem; in Chapter 4, the colloid are prepared such that the citrate-layer is removed prior to addition of dyes, allowing fast electrostatic adsorption with the negative chlorine layer. In many systems of non-spherical particles, colloids are stabilised by larger surface species such as CTAB and PEG. The adsorption time of dyes in these systems may be different that the ones investigated here. Finally the most straightforward extension of the study would be to investigate the phenomenon for a range of other cationic SERS dyes used in the literature, such as Crystal Violet, Malachite Green etc. The individual adsorption kinetics of each dye may play a role in the extent of the non-uniformity of coverages and for some cases the effect may be more or less problematic depending on these kinetics.

In terms of SM-SERS further studies, there are many avenues to pursue towards establishing how fundamental the findings are to routine SM-SERS experiments. The results in Chapter 4 unequivocally highlight that, unless half-half dilutions are used, the dyes play a significant role in creating the clusters from which the largest SERS signals are obtained; this is clearly a less than ideal situation for SM-SERS, where the end goal, from both a fundamental and analytical point of view, is to be able to measure every single-molecule in solution. In fact this was the motivation to move away from nanogaps in Reference [32]. With the half-half method in hand, it should in principle be able to develop a scheme whereby dyes are adsorbed evenly across the colloids, after which aggregation is induced. If this scheme is optimised, the statistics of SM-SERS events should be drastically improved. This is the subject of ongoing work.

Finally the dilution issues highlight that the bi-analyte technique, which is praised for its robustness in identifying single molecule SERS events, has a particular weakness to sample inhomogeneity. To this end, the results of Chapter 4 constitute another significant step in improving the methods used for studying SM-SERS, bringing the technique closer to its potential status as a routine method for ultra-sensitive analysis. 
The second body of work constitutes a similar stepping stone for SERS but arguably from a more fundamental perspective; the role of the chemical enhancement has been a long standing debate in SERS since its discovery in 1974 and the work presented in Chapter 6 opens a new avenue towards studying this effect directly. In the same vain as the dilution study, much of the controversy around EFs has been settled, leaving only a few orders of magnitude left to account for in the total EF that could arise from chemical interactions with the SERS probe and substrate. While the strong-coupling community has raced ahead in the past five years to generate complex hybrid systems of dye-plasmon interactions, the approach in this thesis is motivated by a desire to relate the surface absorbance of the dyes to their SERS properties. This is a fortunate perspective to tackle the problem from because it prompts the need to extract the true dye absorbance rather than that coupled to the plasmon resonance. As such the three main advantages of the approach outlined in Chapter 6 are (1) the dye concentration is kept low such that dye-dye interactions are negligible and the intrinsic surface absorbance can be extracted and (2) the dye-plasmon interaction is minimised by detuning of the resonances, allowing the contributions of plasmon-molecule coupling and adsorption-induced-modifications can be decoupled and (3) the studies are performed in water allowing the surface absorption spectra to be directly compared to the dye absorbance without the metal.

Interpretations have been proposed for the origin of the shifts and further work will be needed to confirm these hypotheses, while the standard opticaltransform model, relating the REP to the molecular absorbance, has been shown to reasonably reproduce the measured SERS REP of CV based on the measured surface absorbance. Crucial to this success was the method established to correctly remove any plasmon resonance dependence of the SERS REP by use of a non-resonant reference molecule.

While the observation of the shifts in absorbance for dyes like Rhodamine $6 \mathrm{G}$ and Rhodamine 700 are indeed interesting for the strong coupling debate, possibly more impactful will be the ability of these experiments to drive theoretical SERS studies further; groups with expertise in the advanced theoretical techniques required to model molecules coupled to metallic structures up to now have limited experimental data based on SERS only to inform their models. An example is that of the studies performed by Morton and Jensen [40], 
where for both RH6G and CV the absorption spectra are predicted to redshift by $\sim 40 \mathrm{~nm}$, which can be directly compared to the results of Chapter 6 require revisiting. With direct access to the electronic absorbances of dye molecules from the CMCIS method, a wealth of new information is available to understand the chemical nature of these shifts.

The work in Chapter 6 has many natural extensions, the first of which is to study the shifts of other families of dye molecules. Of particular interest would be to use the J-aggregates molecules employed in the recent strong coupling $[150,151,164]$ studies to determine if the dye properties change significantly in the absence of any strong coupling. This has the potential to clarify some of the disparate observations of resonance splitting in such systems, which may in fact already exist for dense dye layers on nanoparticles before strong coupling is reached.

A second natural extension is to perform the measurements in Chapter 6 on gold colloids. Such a study would give further insight into the nature of the shifts, in particular for Crystal Violet; if the dramatic shift in the CV spectrum on silver is truly chemical in nature, in principle adsorption to gold would affect the electronic orbitals of the molecule differently, which may modify the $\mathrm{CV}$ absorption spectrum from that on silver. The difficulty with such an experiment is that the plasmon resonance of gold spheres is generally in the 500 to $600 \mathrm{~nm}$ region, which overlaps with the resonance of CV. Other dyes like Malachite Green, that have a similar structure to CV but absorb further in the red so may be useful here in investigating the effect.

From a SERS point of view, there is ample work to be done in terms of investigating the validity of the optical-transform model for molecules other than CV and on substrates other than silver colloids. The optical-transform model could in principle be improved to include non-Condon terms and nonadiabatic approximations that may be necessary to fit experimental data, but this is beyond the scope of the thesis. One of the drawbacks of the CV study is the limited number of laser lines used to measure the SERS REP. It is true that there is one data point missing between the 568 and $633 \mathrm{~nm}$ measurements that would fully drive the point home that the wavelength response of the Raman signal on the metal no longer follows the molecule absorbance in solution. Unfortunately access to a laser line in this region, particularly near the normal 
CV resonance of $590 \mathrm{~nm}$ was not available but would considerably strengthen the current dataset.

Finally, as a general conclusion, it would be naive to suggest that SERS will become a panacea for analytical chemistry, as no technique by itself can solve every scientific problem. However SERS no doubt has some of the most attractive aspects of any analytical technique; sensitivity, down to the single molecule level; specificity to accurately identify and discriminate between analyte species; versatility, in the almost infinite number of ways in which SERS detection can be implemented. Given this potential to revolutionise analytical chemistry, an understanding of the most basic aspects of the technique are of prime importance. This thesis systematically addresses two of these aspects and will contribute significantly to the further advancement of the field. 


\section{Appendix A}

\section{Polarizability of Dyes and Mie Theory Shell Model}

This appendix describes in detail the methods for extracting the polarizabilities of dye molecules from their measured absorbances and how this polarizability can be linked with the dielectric function of a dye layer through the ClausiusMosotti relationship. The details of computing the optical response of dyecoated spheres with Mie-theory as used in Chapter 6 are then presented. ${ }^{1}$

\section{A.1 Polarizability of dyes}

\section{A.1.1 Link between polarizability and absorbance}

In practice, one cannot easily measure the frequency-dependent complex polarizability of a dye, but its imaginary part can be deduced from the absorption cross-section, $\sigma_{\text {abs }}(\omega)$. The latter is readily obtained from a standard UVVis absorbance measurement of the dye in solution at a known concentration. Explicitly, $\sigma_{\text {abs }}(\omega)$ is related to the bare polarizability of the dye $\alpha_{D}(\omega)$ by [16]

$$
\sigma_{\mathrm{abs}}(\omega)=\frac{\left(\epsilon_{M}+2\right)^{2}}{9 \sqrt{\epsilon_{M}}} \frac{\omega}{\varepsilon_{0} c} \operatorname{Im}\left[\alpha_{D}(\omega)\right],
$$

\footnotetext{
${ }^{1}$ Part of the material presented in this Appendix is adapted from the Methods and S.I sections of Reference [128].
} 
where $\epsilon_{M}=n_{M}^{2}$ is the dielectric constant of the surrounding medium (in this study water with $\left.n_{M}=1.33\right), c$ the speed of light in vacuum, $\varepsilon_{0}$ the permittivity of free space. From this, the bare (imaginary) polarizability can be deduced (as a function of wavelength $\lambda=(2 \pi c) / \omega$ for convenience):

$$
\operatorname{Im}\left(\alpha_{D}(\lambda)\right)=\frac{9 \epsilon_{0} \sqrt{\epsilon_{M}}}{\left(\epsilon_{M}+2\right)^{2}} \frac{\lambda}{2 \pi} \sigma_{\text {abs }}(\lambda) .
$$

If the measured absorbance spans a sufficient frequency range, the KramersKrönig relations may be applied to infer the real part of $\alpha_{D}(\lambda)$, possibly up to a constant value accounting for lower-energy transitions. This constant can be determined from a knowledge of the static polarizability, which may be obtained from DFT calculations (explained later).

Note that for simplicity, the polarizability is considered isotropic; a more rigorous derivation could include explicitly the orientation averaging of a uniaxial polarizability tensor, but the same result is obtained by simply replacing $\alpha_{D}$ by $\operatorname{Tr}\left(\hat{\alpha}_{D}\right) / 3$, where $\operatorname{Tr}$ denotes the trace of the tensor. For a uniaxial tensor (the case of most dyes) along the $z$-axis, $\alpha_{D}$ is then replaced by $\alpha_{z z} / 3$ in Eqs. A.2, 1 and 3.

\section{A.1.2 Theoretical fits of bare polarizabilities}

Rather than using the real absorption data to obtain the dielectric function of the dye molecules, it is more convenient to use simplified analytical functions for the polarizability. These can then be used in the Mie-theory shell model (discussed later) to reproduce the experimentally-measured absorbances in the CMCIS setup (Chapter 6). A particularly useful choice of a polarizability model is one that satisfies the Kramers-Krönig relations by construction. A Lorentz oscillator (or a sum of them) is the most commonly used example $[150,151,162-164,167]$.

In the example of Figure 1, a sum of two Lorentz oscillators are used to model the response of a typical dye (chosen to model Rhodamine 6G) with a 
resonance at $526 \mathrm{~nm}$ and a vibronic shoulder at $496 \mathrm{~nm}$ :

$$
\alpha(\lambda)=\alpha_{\text {static }}+\sum_{n=0,1} \frac{\alpha_{n} \lambda_{n}}{\mu_{n}}\left[\frac{1}{1-\frac{\lambda_{n}^{2}}{\lambda}-i \frac{\lambda_{n}^{2}}{\lambda \mu_{n}}}-1\right]
$$

with the following parameters:

$$
\begin{aligned}
\lambda_{0}=526 \mathrm{~nm}, \quad \mu_{0}=10000 \mathrm{~nm}, \quad \alpha_{0} & =5.8 \times 10^{-38} \text { S.I. } \\
\lambda_{1}=496 \mathrm{~nm}, \quad \mu_{1}=6000 \mathrm{~nm}, \quad \alpha_{1} & =1.55 \times 10^{-38} \text { S.I. } \\
\alpha_{\text {static }} & =0.96 \times 10^{-38} \text { S.I. }
\end{aligned}
$$

The parameter $\alpha_{\text {static }}$ (corresponding to the static polarizability, for $\lambda \rightarrow \infty$ ) was obtained from a DFT calculation of Rhodamine 6G (discussed below).

A similar model can be used to describe the polarizability of Rhodamine 700. The parameters $\alpha_{n}, \lambda_{n}$, and $\mu_{n}$ can be determined from a fit to the experimental absorbance spectrum using Eq. A.2 and $\alpha_{\text {static }}$ is again obtained from a DFT calculation. For RH700, the parameters are found to be:

$$
\begin{aligned}
\lambda_{0}=642 \mathrm{~nm}, \quad \mu_{0}=10500 \mathrm{~nm}, \quad \alpha_{0} & =8.15 \times 10^{-38} \text { S.I. } \\
\lambda_{1}=589 \mathrm{~nm}, \quad \mu_{1}=8800 \mathrm{~nm}, \quad \alpha_{1} & =2.8 \times 10^{-38} \text { S.I. } \\
\alpha_{\text {static }} & =1.15 \times 10^{-38} \text { S.I. } .
\end{aligned}
$$

Again, note that most dyes have a strongly uniaxial polarizability tensor at their main electronic resonance. The parameters above correspond to the polarizability along the main axis (i.e. $\alpha_{z z}$ for the $z$-axis) and the corresponding scalar polarizability for orientation averaging is simply $\alpha_{D}=\alpha_{z z} / 3$.

A more accurate model of the spectral lineshape can be obtained by considering alternative analytic functions; indeed, a sum of one or several Lorentzian oscillators rarely provides a good fit to the absorbance of a dye, in particular, for the long-wavelength "wing" of the peaks, despite being Kramers-Krönig consistent. Therefore a continuous sum of Lorentz oscillators inhomogeneously broadened by a Gaussian distribution of oscillator frequencies [159], called a Voigt lineshape, was also considered. Explicitly: 


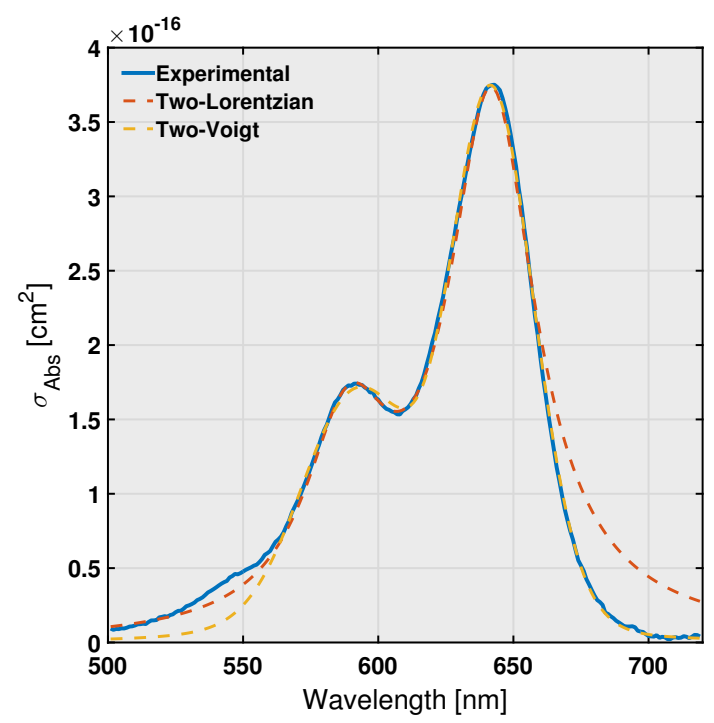

Figure A.1: Fit of the experimental Rhodamine 700 absorbance spectrum, shown in blue. The sum of two Lorentz oscillators (red) shows broader wings. In comparison, the double-Voigt-like lineshape (yellow) gives a more accurate fit.

$$
\alpha(\omega)=\alpha_{\text {static }}+\sum_{n=0,1} \frac{\alpha_{n} \Gamma_{n}}{\omega_{n}}\left[1-\int_{-\infty}^{+\infty} d \omega_{0} \frac{1}{\sigma_{n} \sqrt{2 \pi}} \frac{\exp \left[-\frac{\left(\omega_{0}-\bar{\omega}_{n}\right)^{2}}{2 \sigma_{n}^{2}}\right]}{1-\frac{\omega^{2}}{\omega_{0}^{2}}-i \frac{\omega \Gamma_{n}}{\omega_{0}^{2}}}\right]
$$

The parameters deduced from the absorbance of Rhodamine 700 in water are:

$$
\begin{array}{ll}
2 \pi c / \omega_{0}=642.9 \mathrm{~nm}, & \alpha_{0}=8.5 \times 10^{-37} \text { S.I. } \\
\Gamma_{0} /(2 \pi c)=56.4 \mathrm{~cm}^{-1}, & \sigma_{0} /(2 \pi c)=354.7 \mathrm{~cm}^{-1} \\
2 \pi c / \omega_{1}=593.7 \mathrm{~nm}, & \alpha_{1}=1.17 \times 10^{-37} \text { S.I. } \\
\Gamma_{1} /(2 \pi c)=338.4 \mathrm{~cm}^{-1}, & \sigma_{1} /(2 \pi c)=554.7 \mathrm{~cm}^{-1} \\
\alpha_{\text {static }}=1.15 \times 10^{-38} \text { S.I. } &
\end{array}
$$

As shown in Fig. A.1, this model results in a much better fit of the absorbance of Rhodamine 700 (and other dyes not shown here), and by construction preserves Kramers-Krönig consistency. This is especially better at representing the correct long-wavelength tail of absorption (note also that a third higher energy peak could be added to model the short-wavelength part if needed). 
This analytical expression also proves very useful to model the modified polarizability of adsorbed dyes. For example, for RH700 the modified polarizability shown in Chapter 6, Figure 6.15 was obtained using the same parameters as RH700 in water with the following modifications:

$$
\begin{aligned}
& \omega_{0}^{\prime} /(2 \pi c)=\omega_{0} /(2 \pi c)-620 \mathrm{~cm}^{-1}, \quad \alpha_{0}^{\prime}=1.08 \alpha_{0} \quad \sigma_{0}^{\prime}=1.2 \sigma_{0} \\
& \omega_{1}^{\prime} /(2 \pi c)=\omega_{1} /(2 \pi c)-510 \mathrm{~cm}^{-1}, \quad \alpha_{1}^{\prime}=0.98 \alpha_{1}
\end{aligned}
$$

All the other parameters are unchanged.

Since it is not possible to access the absolute modified polarizability on the nanoparticle surface, the overall scaling of $\alpha_{0}^{\prime}$ and $\alpha_{1}^{\prime}$ was set by conservation of the oscillator strength $f$ of the transitions as calculated from [127]

$$
f=\frac{4.32 \times 10^{-9}}{n_{M}} \int \bar{e}(\bar{\nu}) d \bar{\nu}
$$

where $\bar{\nu}=1 / \lambda$ is the wavenumber in $\mathrm{cm}^{-1}$ and $\bar{e}(\bar{\nu})$ is the decadic molar absorption coefficient in $\mathrm{cm}^{-1} \mathrm{M}^{-1}$.

\section{A.1.3 DFT calculations of static polarizabilities}

Values for $\alpha_{\text {static }}$ were calculated using density functional theory (DFT) ${ }^{2}$. Specifically, the package Gaussian09 [195] was used employing the hybrid functional PBE0 [196, 197] with triple zeta basis set def2tzvp [198, 199] to perform molecular geometry optimizations and polarizability calculations. From the resulting static polarizability tensors (given in the coordinate frame of the molecules) the polarizability component oriented along the dominant axis of the molecule is chosen as representative of the polarizability $\alpha_{\text {static }}$ of the electronic state in the limit of $\lambda \rightarrow \infty$. For example, for Rhodamine 700 the value is $\alpha_{\text {static }}^{\mathrm{Rh} 700}=1.15 \times 10^{-38}$ S.I. and for Rhodamine $6 \mathrm{G} \alpha_{\text {static }}^{\mathrm{Rh} 6 \mathrm{G}}=9.6 \times 10^{-39}$ S.I.

\footnotetext{
${ }^{2}$ DFT calculations were performed by Dr. Matthias Meyer during the preparation of the manuscript for Reference [128]
} 


\section{A.1.4 Clausius-Mossotti Relationship for Dye Layers}

Theoretical predictions of the optical properties of the dye layer were carried out using an isotropic shell model as used in most recent studies of strong coupling in molecular plasmonics $[150,151,159,161,163,164,167,169]$. This consists in solving the electromagnetic scattering problem for a metallic nanosphere covered by a thin spherical shell (of thickness $L_{D}$ ) with an effective dielectric function that models the dye response. Note that $L_{D}$ can here be viewed as an effective thickness, only loosely connected to the physical size of the dye molecule, i.e. it is an adjustable parameter (whose meaning is defined more precisely in Ref. [181]). In order to study the dye concentration dependence, it is necessary to start from the polarizability of the individual dyes and account for local field corrections using the Clausius-Mossotti (CM) relation in the 2D dense dye layer, as one would do in a bulk 3D phase. This has been studied in the past [181] for dyes in vacuum and has been adapted to the case where the dyes are embedded in a medium, here water with a dielectric constant $\epsilon_{M}=1.77$. The effective dielectric function of the dye layer is then

$$
\epsilon_{\mathrm{dye}}=\frac{1+\frac{2}{3}\left(\tilde{\alpha}_{M}+\tilde{\alpha}_{D}\right)}{1-\frac{1}{3}\left(\tilde{\alpha}_{M}+\tilde{\alpha}_{D}\right)}
$$

where $\tilde{\alpha}_{M}$ and $\tilde{\alpha}_{D}$ are the normalized bare polarizabilities of the solvent (water) and dye molecules. The former is simply deduced from the standard CM equation [200] in a pure phase,

$$
\tilde{\alpha}_{M}=3 \frac{\epsilon_{M}-1}{\epsilon_{M}+2}(\approx 0.61)
$$

The latter depends directly on the density of dyes in the shell layer, via the concentration of dyes, $c_{D}$ (note that this is related to the number of dyes per unit area $\mu_{D}$ by $\left.c_{D} \approx \mu_{D} / L_{D}\right)$ :

$$
\tilde{\alpha}_{D}(\omega)=c_{D} \frac{\alpha_{D}}{\epsilon_{0}}
$$

This approach then correctly accounts for the change in optical properties of the dye layer as the dye density increases, as was illustrated in Chapter 6, 
Figure 6.10 (a) for a fictitious dye-coated-water-sphere. It is now clear why the value of $L_{\mathrm{D}}$ is plays an important role in reproducing the experimental results. The choice of $L_{\mathrm{D}}$ determines the strength of interaction between dye molecules in the dye layer and can be tuned in the model to match the observed spectral changes with increasing dye concentration.

The bare polarizability $\alpha_{D}(\omega)$ is deduced from the fitting procedure described in Section A.1.2 using the measured optical absorbance of the dye. The the static polarizability is calculated by DFT as described previously.

\section{A.1.5 Mie-Theory Model of Dye-Coated Spheres}

The electromagnetic problem is then solved using Mie theory for multilayered spheres under plane wave excitation using standard MATLAB codes [69] and as depicted if Figure A.2 below.

As mentioned in Chapter 2, the derivation and implementation of the Mietheory equations are discussed in detail in Refs. [16,68] and only the most relevant equations are presented here. The goal of Mie-theory is essentially to solve the electromagnetic problem for scattering by a homogeneous, isotropic, non-magnetic sphere in a non-absorbing medium excited by an external incident field. Following the treatment in Reference [16], the approach is, through imposition of the appropriate boundary conditions, to find the fields inside and outside the sphere. The field inside the sphere is denoted $\mathbf{E}_{\text {In }}$ while the field outside the sphere can be written as

$$
\mathbf{E}_{\mathrm{out}}=\mathbf{E}_{\mathrm{Inc}}+\mathbf{E}_{\mathrm{Sca}} \text {. }
$$

Im Mie-theory, all fields are written in spherical coordinates. As such, each field can be expanded as a sum of vector spherical harmonics, $\mathbf{M}_{n m}^{(i)}$ and $\mathbf{N}_{n m}^{(i)}$, for the magnetic and electric components of the field respectively. Physically, 


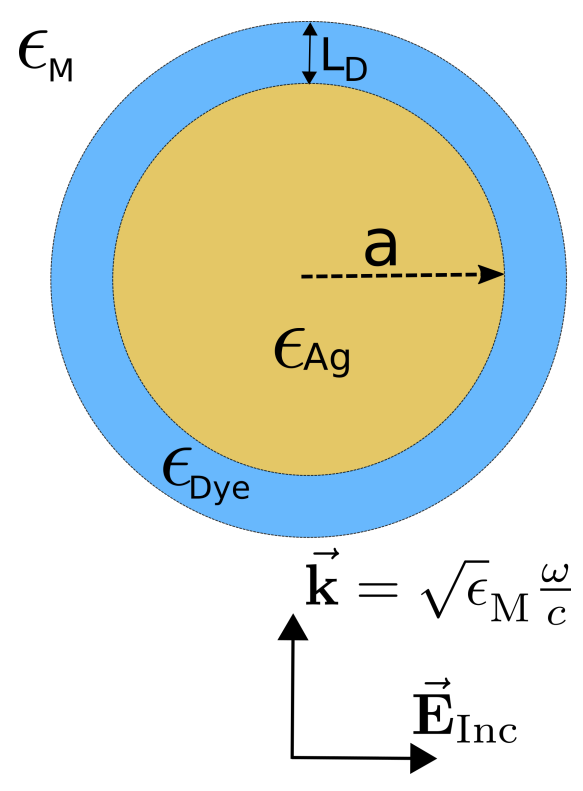

Figure A.2: Schematic of the dye-coated-sphere model used to predict the optical properties of a dye-layer surrounding a $60 \mathrm{~nm}$ diameter silver nanoparticle embedded in water $\left(\epsilon_{M}=1.77\right)$. The problem is solved in Mie-theory, here for a plane-wave incident electric field polarised along the $x$-axis propagating along the $z$-axis as shown.

these vector spherical harmonics are the fields created by a multipolar distribution of sources, where the indices $n$ and $m$ indicate the order of the electric and magnetic component in the field expansion. Simply put, $n=1$ corresponds to the dipole component of the field, $n=2$ the quadrupole moment and so on. The VSHs are functions that describe the electric and magnetic field in terms of $r, \theta$ and $\phi$. The radial dependence is described by spherical Bessel functions and the angular by spherical harmonics. The index $i$ denotes the type of VSH, ranging from $i=1 \ldots .4$, which determines the type of spherical Bessel used to describe that component of the electric field. This is discussed in more detail in [68]. Each field is then written as:

$$
\begin{aligned}
& \mathbf{E}_{\mathrm{Inc}}(\mathbf{r})=E_{0} \sum_{n, m} a_{n m} \mathbf{M}_{n m}^{(1)}\left(k_{M}, \mathbf{r}\right)+b_{n m} \mathbf{N}_{n m}^{(1)}\left(k_{M}, \mathbf{r}\right), \\
& \mathbf{E}_{\mathrm{Sca}}(\mathbf{r})=E_{0} \sum_{n, m} c_{n m} \mathbf{M}_{n m}^{(3)}\left(k_{M}, \mathbf{r}\right)+d_{n m} \mathbf{N}_{n m}^{(3)}\left(k_{M}, \mathbf{r}\right),
\end{aligned}
$$

and 


$$
\mathbf{E}_{\text {In }}(\mathbf{r})=E_{0} \sum_{n, m} \alpha_{n m} \mathbf{M}_{n m}^{(1)}\left(k_{M}, \mathbf{r}\right)+\beta_{n m} \mathbf{N}_{n m}^{(1)}\left(k_{M}, \mathbf{r}\right)
$$

The coefficients $a_{n m}, b_{n m}, c_{n m}, d_{n m}, \alpha_{n m}$ and $b_{n m}$ are known as the Mie coefficients that essentially relate the incident field (of which the components $a_{n m}$ and $b_{n m}$ are known) to the scattered and internal field. The problem is then solved for by applying the standard EM boundary conditions which results in a series of linear equations that can be solved for the unknown coefficients to compute the scattered and inside field.

Due to the linearity of Maxwell's equations, the scattered and internal field coefficients are proportional to the incident field coefficients. For the purposes of this thesis, only the scattered field is of interest, and as such it is related to the incident field by:

$$
\begin{gathered}
c_{n m}=\Gamma_{n} a_{n m} \\
d_{n m}=\Delta_{n} b_{n m} .
\end{gathered}
$$

Equations A.13 and A.14 characterise the optical and magnetic response of the sphere, where $\Gamma_{n}$ and $\Delta_{n}$ are referred to as the spheres (frequency dependent) magnetic and electric susceptibilities. Therefore, these two functions are of crucial importance in Mie-theory, as they determine the optical and magnetic properties of the sphere. They are expressed as:

$$
\Gamma_{n}=\frac{s \psi_{n}(x) \psi_{n}^{\prime}(s x)-\psi_{n}(s x) \psi_{n}^{\prime}(x)}{\psi_{n}(s x) \xi_{n}^{\prime}(x)-s \xi_{n}(x) \psi_{n}^{\prime}(s x)},
$$

and

$$
\Delta_{n}=\frac{\psi_{n}(x) \psi_{n}^{\prime}(s x)-s \psi_{n}(s x) \psi_{n}^{\prime}(x)}{s \psi_{n}(s x) \xi_{n}^{\prime}(x)-\xi_{n}(x) \psi_{n}^{\prime}(s x)}
$$

Here the (adimensional) variables $x$ and $s$ are defined as: 


$$
x=2 \pi \sqrt{\epsilon_{M}} \frac{a}{\lambda} \quad \text { and } \quad s=\frac{\sqrt{\epsilon_{i n}}}{\sqrt{\epsilon_{M}}}
$$

while $\psi_{n}$ and $\xi_{n}$ are the Riccatti-Bessel functions given by:

$$
\psi_{n}(\rho)=\rho j_{n}(\rho) \quad \text { and } \quad \xi_{n}(\rho)=\rho h_{n}^{(1)}(\rho) .
$$

Finally, the parameters of interest experimentally to measuring colloidal solutions, namely the extinction, scattering and absorption cross sections can be written as [16] (here normalized to the sphere geometrical cross section):

$$
Q_{\mathrm{Sca}}^{\mathrm{NP}}=\frac{\sigma_{\mathrm{Sca}}}{\pi a^{2}}=\frac{2}{x^{2}} \sum_{n=1}^{\infty}(2 n+1)\left(\left|\Gamma_{n}\right|^{2}+\left|\Delta_{n}\right|^{2}\right) .
$$

Similarly the extinction cross section is given as:

$$
Q_{\mathrm{Ext}}^{\mathrm{NP}}=\frac{\sigma_{\mathrm{Ext}}}{\pi a^{2}}=\frac{-2}{x^{2}} \sum_{n=1}^{\infty}(2 n+1)\left[\operatorname{Re}\left(\Gamma_{n}\right)+\operatorname{Re} \Delta_{n}\right]
$$

Given that extinction is the sum of scattering and absorption, the absorption cross section is then given simply by:

$$
Q_{\mathrm{Abs}}^{\mathrm{NP}}=Q_{\mathrm{Ext}}^{\mathrm{NP}}-Q_{\mathrm{Sca}}^{\mathrm{NP}}
$$

The three quantities above fully characterise what can be measured experimentally by UV-Vis spectroscopy for a solution of colloids and are the basis of determining both the size and concentration of colloidal solutions by comparing the predicted spectra to those measured.

The above approach is for a single, uncoated sphere but can in principle be extended to coated spheres, as depicted in Figure A.2. This is performed in the same way by representing the fields in each region (the core particle, the shell, and outside) by VSHs and solving for the scattering coefficients in each region by applying the appropriate boundary conditions at the interface between each 
region. This results in more unknown coefficients than the single sphere but the proportionality relationship between the scattered and incident field is the same. The coefficients are then solved for recursively at each surface. This is outlined in more detail in Appendix H of Reference [16].

The problem is implemented in the SpLaC codes by specifying the dye layer thickness (which in the case here is $L_{\mathrm{D}}$ ) and the wavelength dependent dielectric function of the layer, given by Equation A.1.4 and the core particle (silver), along with the radius of the core particle and the dielectric constant of the embedding medium.

The wavelength dependent, complex dielectric function of silver is taken from the analytical fit to various experimental data of the optical properties of silver [61,201] derived in Reference [16] given by:

$$
\epsilon_{A g}(\lambda)=\epsilon_{\infty}\left(1-\frac{1}{\lambda_{p}^{2}\left(\frac{1}{\lambda^{2}}+\frac{i}{\mu_{p} \lambda}\right)}\right)
$$

for which the best fit to the experimental data were found to be $\epsilon_{\infty}=4$, $\lambda_{p}=282 \mathrm{~nm}$ and $\mu_{p}=17000 \mathrm{~nm}$.

The response of dye-coated spheres can now be calculated within the Mietheory framework by a simply taking difference between coated sphere and the bare sphere cross sections (extinction, scattering and absorption), yielding the response of the dye-layer. The results of this approach are shown in Chapter 6 but for clarity are repeated here. Figure A.3 illustrates this approach in a simple case. In (a), the bare responses of uncoated $30 \mathrm{~nm}$ radius $\mathrm{Ag}$ spheres are shown. In (b) the dye layer was modelled as Rhodamine 6G (obtained from the Clausius-Mossotti formalism and Voigt-fits to the measured absorption spectrum) at an artificially high concentration of $25 \mathrm{nM}$, with a shell thickness of $0.2 \mathrm{~nm}$ (or 3.15 dyes $/ \mathrm{nm}^{3}$ ). In (c) the bare NP responses are subtracted from the coated responses, to obtain the differential absorption and extinction spectra as described in Chapter 6. Also plotted is the spectrum of the bare dye in solution for comparison. Note the spectra are scaled by the real concentration and thus are plotted as real absorbances rather than as cross-sections as given by Equations A.19, A.21 and A.20. $\left\langle\mathrm{M}_{\mathrm{loc}} \lambda\right\rangle$ is calculated for the NP-only 
model, by taking the average of the field intensity enhancement factor on the spherical surface in the middle of the thin shell. 


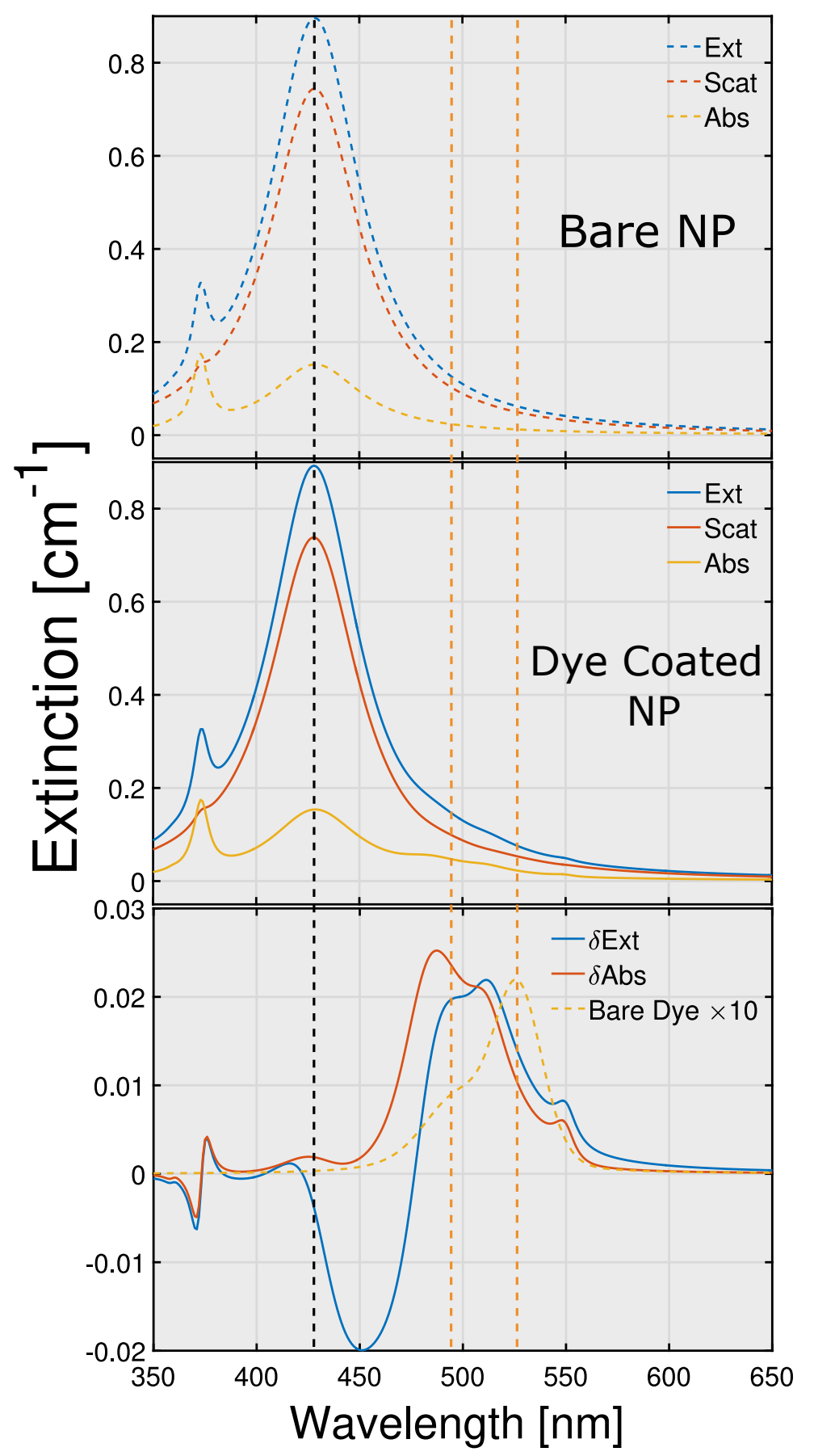

Figure A.3: The steps involved in computing the optical response of the dye layer in the Mie-theory shell model used in Chapter 6. The predicted extinction scattering and absorption spectra are shown for (top) uncoated and (middle) coated $30 \mathrm{~nm}$ radius silver spheres in water. The dye layer thickness was set to $0.2 \mathrm{~nm}$ and the dye concentration is $25 \mathrm{nM}$. The dye layer properties are obtained by subtraction of the top panel spectra from the middle panel spectra to give (bottom) the differential extinction and absorbance spectra. The bare dye spectrum (orange dashed line) is shown for comparison. 


\section{Bibliography}

[1] Fleischmann, M., Hendra, P. J., \& McQuillan, A. Raman spectra of pyridine adsorbed at a silver electrode. Chemical Physics Letters 26(2), 163-166 (1974).

[2] Kneipp, K., Wang, Y., Kneipp, H., Perelman, L. T., Itzkan, I., Dasari, R. R., \& Feld, M. S. Single molecule detection using surface-enhanced Raman scattering (SERS). Physical Review Letters 78(9), 1667 (1997).

[3] Nie, S. \& Emory, S. R. Probing single molecules and single nanoparticles by surface-enhanced Raman scattering. Science 275(5303), 1102-1106 (1997).

[4] Jeanmaire, D. L. \& Van Duyne, R. P. Surface Raman spectroelectrochemistry: Part i. heterocyclic, aromatic, and aliphatic amines adsorbed on the anodized silver electrode. Journal of Electroanalytical Chemistry and Interfacial Electrochemistry 84(1), 1-20 (1977).

[5] Albrecht, M. G. \& Creighton, J. A. Anomalously intense Raman spectra of pyridine at a silver electrode. Journal of the American Chemical Society 99(15), 5215-5217 (1977).

[6] Timper, J., Billmann, J., Otto, A., \& Pockrand, I. Surface enhanced light scattering from silver electrodes: Background and CN stretch vibration. Surface Science 101(1), 348-354 (1980).

[7] McCall, S. \& Platzman, P. Raman scattering from chemisorbed molecules at surfaces. Physical Review B 22, 1660-1662 (1980).

[8] Ke-hsueh, L. A model of coherent parametric excitation for giant Raman effect. Surface Science 115(3), 513-523 (1982). 
[9] Moskovits, M. Surface roughness and the enhanced intensity of Raman scattering by molecules adsorbed on metals. The Journal of Chemical Physics 69(9), 4159-4161 (1978).

[10] Moskovits, M. Surface-enhanced spectroscopy. Rev. Mod. Phys. 57(3), 783-826 (1985).

[11] Kiefer, W. \& Schlücker, S. Surface enhanced Raman spectroscopy: analytical, biophysical and life science applications. Wiley. com, (2011).

[12] Le Ru, E. C., Blackie, E., Meyer, M., \& Etchegoin, P. G. Surface enhanced Raman scattering enhancement factors: a comprehensive study. J. Phys. Chem. C 111, 13794-13803 (2007).

[13] Moerner, W. E. \& Kador, L. Optical detection and spectroscopy of single molecules in a solid. Phys. Rev. Lett. 62, 2535-2538, May (1989).

[14] Ambrose, W. P., Goodwin, P. M., Jett, J. H., Van Orden, A., Werner, J. H., \& Keller, R. A. Single molecule fluorescence spectroscopy at ambient temperature. Chemical Reviews 99(10), 2929-2956 (1999).

[15] Arroyo, J. O. \& Kukura, P. Non-fluorescent schemes for single-molecule detection, imaging and spectroscopy. Nature Photonics 10(1), 11-17 (2016).

[16] Le Ru, E. C. \& Etchegoin, P. G. Principles of Surface Enhanced Raman Spectroscopy and Related Plasmonic Effects. Elsevier, Amsterdam, (2009).

[17] Artur, C. G., Miller, R., Meyer, M., Le Ru, E. C., \& Etchegoin, P. G. Single-molecule sers detection of c 60. Physical Chemistry Chemical Physics 14(9), 3219-3225 (2012).

[18] Etchegoin, P. G., Le Ru, E. C., \& Meyer, M. Evidence of natural isotopic distribution from single-molecule sers. Journal of the American Chemical Society 131(7), 2713-2716 (2009).

[19] Le Ru, E. C. \& Etchegoin, P. G. Single molecule surfaced-enhanced Raman spectroscopy. Annu. Rev. Phys. Chem. 63, 65-87 (2012).

[20] Le Ru, E. C. \& Etchegoin, P. G. Quantifying SERS enhancements. MRS Bulletin 38, 631-640 (2013). 
[21] Natan, M. J. Concluding remarks: surface enhanced Raman scattering. Faraday Discuss. 132, 321-328 (2006).

[22] Faulds, K., Smith, W. E., \& Graham, D. Evaluation of surface-enhanced resonance Raman scattering for quantitative DNA analysis. Anal. Chem. 76(2), 412-417 (2004).

[23] Ackermann, K. R., Henkel, T., \& Popp, J. Quantitative online detection of low-concentrated drugs via a SERS microfluidic system. ChemPhysChem 8(18), 2665-2670 (2007).

[24] Bell, S. E. J. \& Sirimuthu, N. M. S. Quantitative surface-enhanced Raman spectroscopy. Chem. Soc. Rev. 37, 1012-1024 (2008).

[25] Kasera, S., Biedermann, F., Baumberg, J. J., Scherman, O. A., \& Mahajan, S. Quantitative SERS using the sequestration of small molecules inside precise plasmonic nanoconstructs. Nano Lett. 12, 5924-5928 (2012).

[26] Zhang, D., Xie, Y., Deb, S. K., Davison, V. J., , \& Ben-Amotz, D. Isotope edited internal standard method for quantitative surface-enhanced Raman spectroscopy. Anal. Chem. 77, 3563-3569 (2005).

[27] Etchegoin, P. G. \& Le Ru, E. C. A perspective on single molecule SERS: Current status and future challenges. Phys. Chem. Chem. Phys. 10, 6079-6089 (2008).

[28] Pieczonka, N. P. W. \& Aroca, R. F. Single molecule analysis by surfacedenhanced Raman scattering. Chem. Soc. Rev. 37, 946-954 (2008).

[29] Le Ru, E. C., Etchegoin, P. G., \& Meyer, M. Enhancement factor distribution around a single SERS hot-spot and its relation to single molecule detection. J. Chem. Phys. 125, 204701 (2006).

[30] Fang, Y., Seong, N.-H., \& Dlott, D. D. Measurement of the distribution of site enhancements in surface-enhanced Raman scattering. Science 321, 388-391 (2008).

[31] Jiang, J., Bosnick, K., Maillard, M., \& Brus, L. Single molecule Raman spectroscopy at the junctions of large Ag nanocrystals. J. Phys. Chem. B 107(37), 9964-9972 (2003). 
[32] Le Ru, E. C., Grand, J., Sow, I., Somerville, W. R. C., Etchegoin, P. G., Treguer-Delapierre, M., Charron, G., Felidj, N., Levi, G., \& Aubard, J. A scheme for detecting every single target molecule with surface-enhanced Raman spectroscopy. Nano Lett. 11, 5013 (2011).

[33] Jain, P. K., Huang, W., \& El-Sayed, M. A. On the universal scaling behavior of the distance decay of plasmon coupling in metal nanoparticle pairs: A plasmon ruler equation. Nano Lett. 7, 2080-2088 (2007).

[34] Otto, A. The chemical(electronic) contribution to surface-enhanced Raman scattering. Journal of Raman Spectroscopy 36(6-7), 497-509 (2005).

[35] Otto, A., Billmann, J., Eickmans, J., Ertürk, U., \& Pettenkofer, C. The adatom model of sers (surface enhanced Raman scattering): the present status. Surface Science 138(2), 319-338 (1984).

[36] Otto, A. What is observed in single molecule sers, and why? Journal of Raman Spectroscopy 33(8), 593-598 (2002).

[37] Moskovits, M. Persistent misconceptions regarding sers. Physical Chemistry Chemical Physics 15(15), 5301-5311 (2013).

[38] Schatz, G. C., Young, M. A., \& Van Duyne, R. P. Electromagnetic mechanism of SERS. In Surface-enhanced Raman scattering, 19-45. Springer (2006).

[39] Guerrini, L. \& Graham, D. Molecularly-mediated assemblies of plasmonic nanoparticles for surface-enhanced Raman spectroscopy applications. Chemical Society Reviews 41(21), 7085-7107 (2012).

[40] Morton, S. M. \& Jensen, L. A discrete interaction model/quantum mechanical method for describing response properties of molecules adsorbed on metal nanoparticles. The Journal of Chemical Physics 133(7), 074103 (2010).

[41] Uetsuki, K., Verma, P., Yano, T.-a., Saito, Y., Ichimura, T., \& Kawata, S. Experimental identification of chemical effects in surface enhanced Raman scattering of 4-aminothiophenol. The Journal of Physical Chemistry $C$ 114(16), 7515-7520 (2010). 
[42] Valley, N., Greeneltch, N., Van Duyne, R. P., \& Schatz, G. C. A look at the origin and magnitude of the chemical contribution to the enhancement mechanism of surface-enhanced Raman spectroscopy (SERS): Theory and experiment. The Journal of Physical Chemistry Letters 4(16), 2599-2604 (2013).

[43] Lee, P. C. \& Meisel, D. Adsorption and surface-enhanced Raman of dyes on silver and gold sols. J. Phys. Chem. 86, 3391-3395 (1982).

[44] Galloway, C. M., Artur, C., Grand, J., \& Le Ru, E. C. Photobleaching of fluorophores on the surface of nanoantennas. The Journal of Physical Chemistry C 118(49), 28820-28830 (2014).

[45] Zrimsek, A. B., Henry, A.-I., \& Van Duyne, R. P. Single molecule surfaceenhanced Raman spectroscopy without nanogaps. The Journal of Physical Chemistry Letters 4(19), 3206-3210 (2013).

[46] Cialla, D., März, A., Böhme, R., Theil, F., Weber, K., Schmitt, M., \& Popp, J. Surface-enhanced Raman spectroscopy (SERS): progress and trends. Analytical and Bioanalytical Chemistry 403(1), 27-54 (2012).

[47] Gracie, K., Moores, M., Smith, W. E., Harding, K., Girolami, M., Graham, D., \& Faulds, K. Preferential attachment of specific fluorescent dyes and dye labelled dna sequences in a SERS multiplex. Analytical Chemistry (2015).

[48] Marks, H., Mabbott, S., Jackson, G. W., Graham, D., \& Cote, G. L. SERS active colloidal nanoparticles for the detection of small blood biomarkers using aptamers. In SPIE BiOS, 93381C-93381C. International Society for Optics and Photonics, (2015).

[49] Dick, S., Konrad, M. P., Lee, W. W., McCabe, H., McCracken, J. N., Rahman, T., Stewart, A., Xu, Y., \& Bell, S. E. Surface-enhanced Raman spectroscopy as a probe of the surface chemistry of nanostructured materials. Advanced Materials (2016).

[50] Grzelczak, M., Pérez-Juste, J., Mulvaney, P., \& Liz-Marzán, L. M. Shape control in gold nanoparticle synthesis. Chemical Society Reviews 37(9), 1783-1791 (2008). 
[51] Langer, J., Novikov, S. M., \& Liz-Marzán, L. M. Sensing using plasmonic nanostructures and nanoparticles. Nanotechnology 26(32), 322001 (2015).

[52] Samal, A. K., Polavarapu, L., Rodal-Cedeira, S., Liz-Marzán, L. M., Pérez-Juste, J., \& Pastoriza-Santos, I. Size tunable Au@Ag core-shell nanoparticles: synthesis and surface-enhanced Raman scattering properties. Langmuir 29(48), 15076-15082 (2013).

[53] Thacker, V. V., Herrmann, L. O., Sigle, D. O., Zhang, T., Liedl, T., Baumberg, J. J., \& Keyser, U. F. DNA origami based assembly of gold nanoparticle dimers for surface-enhanced Raman scattering. Nature Communications 5 (2014).

[54] Long, D. The Raman Effect: A Unified Treatment of the Theory of Raman Scattering by Molecules. Wiley, (2002).

[55] Turro, N. Modern Molecular Photochemistry. University Science Books, (1991).

[56] McCreery, R. L. Raman Spectroscopy for Chemical Analysis. John Wiley \& Sons, (2005).

[57] Fox, A. M. Optical Properties of Solids. Oxford University Press, USA, (2001).

[58] Raman, C. V. \& Krishnan, K. S. A new type of secondary radiation. Nature 121, 501-502 (1928).

[59] Smekal, A. Zur quantentheorie der dispersion. Naturwissenschaften 11(43), 873-875 (1923).

[60] Shim, S., Stuart, C. M., \& Mathies, R. A. Resonance Raman crosssections and vibronic analysis of Rhodamine $6 \mathrm{G}$ from broadband stimulated Raman spectroscopy. ChemPhysChem 9(5), 697-699 (2008).

[61] Palik, E. D. Handbook of Optical Constants of Solids. Academic Press, (1998).

[62] Etchegoin, P. G., Le Ru, E., \& Meyer, M. An analytic model for the optical properties of gold. The Journal of Chemical Physics 125(16), 164705 (2006). 
[63] Jackson, J. D. Classical Electrodynamics. Wiley, (1999).

[64] Maier, S. A. Plasmonics: Fundamentals and Applications. Springer Science \& Business Media, (2007).

[65] Mie, G. Beiträge zur optik trüber medien, speziell kolloidaler metallösungen. Annalen der Physik 330(3), 377-445 (1908).

[66] Le Ru, E. \& Etchegoin, P. Rigorous justification of the $|E|^{4}$ enhancement factor in surface enhanced Raman spectroscopy. Chemical Physics Letters 423(1), 63-66 (2006).

[67] Mishchenko, M. I., Travis, L. D., \& Lacis, A. A. Scattering, Absorption, and Emission of Light by Small Particles. Cambridge University Press, (2002).

[68] Bohren, C. F. \& Huffman, D. R. Absorption and Scattering of Light by Small Particles. John Wiley \& Sons, (2008).

[69] Le Ru, E. C. \& Etchegoin, P. G. SERS and plasmonics codes (SPlaC). Matlab codes freely available from http://www.vuw.ac.nz/raman/book/codes.aspx.

[70] Zrimsek, A. B., Henry, A.-I., \& Van Duyne, R. P. Single molecule surfaceenhanced Raman spectroscopy without nanogaps. J. Phys. Chem. Lett. 4(19), 3206-3210 (2013).

[71] Le Ru, E. C., Galloway, C., \& Etchegoin, P. G. On the connection between optical absorption/extinction and SERS enhancements. Phys. Chem. Chem. Phys. 8, 3083-3087 (2006).

[72] Blackie, E. Quantification of the Enhancement Factor in SurfaceEnhanced Raman Scattering. PhD thesis, Victoria University of Wellington, (2010).

[73] Bell, S. E. \& Sirimuthu, N. M. Surface-enhanced Raman spectroscopy as a probe of competitive binding by anions to citrate-reduced silver colloids. The Journal of Physical Chemistry A 109(33), 7405-7410 (2005).

[74] Meyer, M., Le Ru, E. C., \& Etchegoin, P. G. Self-limiting aggregation leads to long-lived metastable clusters in colloidal solutions. J. Phys. Chem. B 110, 6040-6047 (2006). 
[75] Bell, S. E. \& Sirimuthu, N. M. Surface-enhanced Raman spectroscopy as a probe of competitive binding by anions to citrate-reduced silver colloids. The Journal of Physical Chemistry A 109(33), 7405-7410 (2005).

[76] Schäfer, F. Dye Lasers. Topics in Applied Physics. Springer Berlin Heidelberg, (2013).

[77] Beija, M., Afonso, C. A., \& Martinho, J. M. Synthesis and applications of Rhodamine derivatives as fluorescent probes. Chemical Society Reviews 38(8), 2410-2433 (2009).

[78] Drexhage, K. H. Structure and properties of laser dyes. In Dye lasers, 144-193. Springer (1973).

[79] Meyer, S. A., Ru, E. C. L., \& Etchegoin, P. G. Quantifying resonant Raman cross sections with sers. The Journal of Physical Chemistry A 114(17), 5515-5519 (2010).

[80] Le Ru, E. C., Schroeter, L. C., \& Etchegoin, P. G. Direct measurement of resonance Raman spectra and cross sections by a polarization difference technique. Analytical Chemistry 84(11), 5074-5079 (2012).

[81] Reigue, A., Auguié, B., Etchegoin, P. G., \& Le Ru, E. C. Cw measurements of resonance Raman profiles, line-widths, and cross-sections of fluorescent dyes: application to Nile Blue A in water and ethanol. Journal of Raman Spectroscopy 44(4), 573-581 (2013).

[82] Le Ru, E., Meyer, S., Artur, C., Etchegoin, P., Grand, J., Lang, P., \& Maurel, F. Experimental demonstration of surface selection rules for SERS on flat metallic surfaces. Chemical Communications 47(13), 39033905 (2011).

[83] Babendure, J. R., Adams, S. R., \& Tsien, R. Y. Aptamers switch on fluorescence of triphenylmethane dyes. Journal of the American Chemical Society 125(48), 14716-14717 (2003).

[84] Langford, N., Smith, K., Sibbett, W., \& Taylor, J. The hybrid modelocking of a CW Rhodamine 700 dye laser. Optics Communications 58(1), 56-58 (1986). 
[85] Kolmakov, K., Belov, V. N., Bierwagen, J., Ringemann, C., Müller, V., Eggeling, C., \& Hell, S. W. Red-emitting Rhodamine dyes for fluorescence microscopy and nanoscopy. Chemistry-A European Journal 16(1), 158-166 (2010).

[86] Le Ru, E. C., Meyer, M., \& Etchegoin, P. G. Proof of single-molecule sensitivity in surface enhanced Raman scattering (SERS) by means of a two-analyte technique. J. Phys. Chem. B 110, 1944-1948 (2006).

[87] Etchegoin, P. G., Meyer, M., \& Le Ru, E. Statistics of single molecule SERS signals: is there a Poisson distribution of intensities? Physical Chemistry Chemical Physics 9(23), 3006-3010 (2007).

[88] Sigle, D. O., Kasera, S., Herrmann, L. O., Palma, A., de Nijs, B., Benz, F., Mahajan, S., Baumberg, J. J., \& Scherman, O. A. Observing single molecules complexing with cucurbit[7]uril through nanogap-SERS. $J$. Phys. Chem. Lett (2016).

[89] Patra, P. P. \& Kumar, G. P. Single-molecule surface-enhanced Raman scattering sensitivity of Ag-core Au-shell nanoparticles: revealed by bianalyte method. J. Phys. Chem. Lett 4(7), 1167-1171 (2013).

[90] Zrimsek, A. B., Wong, N. L., \& Van Duyne, R. P. Single molecule surfaceenhanced Raman spectroscopy: A critical analysis of the bianalyte vs. isotopologue proof. The Journal of Physical Chemistry C (2016).

[91] Blackie, E., Le Ru, E. C., Meyer, M., Timmer, M., Burkett, B., Northcote, P., \& Etchegoin, P. G. Bi-analyte SERS with isotopically edited dyes. Phys. Chem. Chem. Phys. 10, 4147-4153 (2008).

[92] Darby, B. L. \& Le Ru, E. C. Competition between molecular adsorption and diffusion: Dramatic consequences for SERS in colloidal solutions. $J$. Am. Chem. Soc. 136, 10965-10973 (2014).

[93] Sivapalan, S. T., DeVetter, B. M., Yang, T. K., van Dijk, T., Schulmerich, M. V., Carney, P. S., Bhargava, R., \& Murphy, C. J. Offresonance surface-enhanced Raman spectroscopy from gold nanorod suspensions as a function of aspect ratio: not what we thought. ACS nano 7(3), 2099-2105 (2013). 
[94] Bell, S. E. \& McCourt, M. R. SERS enhancement by aggregated Au colloids: effect of particle size. Phys. Chem. Chem. Phys. 11(34), 74557462 (2009).

[95] Futamata, M. \& Maruyama, Y. LSP spectral changes correlating with SERS activation and quenching for R6G on immobilized Ag nanoparticles. Appl. Phys. B 93(1), 117-130 (2008).

[96] Futamata, M., Yu, Y., \& Yajima, T. Elucidation of electrostatic interaction between cationic dyes and Ag nanoparticles generating enormous SERS enhancement in aqueous solution. J. Phys. Chem. Soc. 115, 52715279 (2011).

[97] Gendron, P.-O., Avaltroni, F., \& Wilkinson, K. Diffusion coefficients of several Rhodamine derivatives as determined by pulsed field gradientnuclear magnetic resonance and fluorescence correlation spectroscopy. Journal of Fluorescence 18(6), 1093-1101 (2008).

[98] Galloway, C., Etchegoin, P., \& Le Ru, E. Ultrafast nonradiative decay rates on metallic surfaces by comparing surface-enhanced Raman and fluorescence signals of single molecules. Physical Review Letters 103(6), 063003 (2009).

[99] Pierre, M. C. S., Mackie, P. M., Roca, M., \& Haes, A. J. Correlating molecular surface coverage and solution-phase nanoparticle concentration to surface-enhanced Raman scattering intensities. J. Phys. Chem. C 115, 18511-18517 (2011).

[100] Schwartzberg, A. M., Grant, C. D., Wolcott, A., Talley, C. E., Huser, T. R., Bogomolni, R., \& Zhang, J. Z. Unique gold nanoparticle aggregates as a highly active surface-enhanced Raman scattering substrate. J. Phys. Chem. B 108, 19191-19197 (2004).

[101] Taylor, R. W., Lee, T.-C., Scherman, O. A., Esteban, R., Aizpurua, J., Huang, F. M., Baumberg, J. J., \& Mahajan, S. Precise subnanometer plasmonic junctions for SERS within gold nanoparticle assemblies using cucurbit[n]uril "glue". ACS Nano 5, 3878-3887 (2011). 
[102] Shaw, C. P., Fan, M., Lane, C., Barry, G., Jirasek, A. I., \& Brolo, A. G. Statistical correlation between SERS intensity and nanoparticle cluster size. J. Phys. Chem. C 117, 16596-16605 (2013).

[103] Lebovka, N. I. Aggregation of charged colloidal particles. Adv. Polym. Sci. 255, 57-96 (2014).

[104] Xu, H., Bjerneld, E. J., Käll, M., \& Börjesson, L. Spectroscopy of single hemoglobin molecules by surface enhanced Raman scattering. Phys. Rev. Lett. 83, 4357-4360 (1999).

[105] Goulet, P. J. \& Aroca, R. F. Distinguishing individual vibrational fingerprints: single-molecule Surface-Enhanced Resonance Raman scattering from one-to-one binary mixtures in Langmuir-Blodgett monolayers. Anal. Chem. 79(7), 2728-2734 (2007).

[106] Titus, E. J., Weber, M. L., Stranahan, S. M., \& Willets, K. A. Superresolution SERS imaging beyond the single-molecule limit: An isotopeedited approach. Nano Lett. 12(10), 5103-5110 (2012).

[107] Etchegoin, P. G., Meyer, M., Blackie, E., \& Le Ru, E. C. Statistics of single-molecule surface enhanced Raman scattering signals: fluctuation analysis with multiple analyte techniques. Anal. Chem. 79, 8411-8515 (2007).

[108] Dieringer, J. A., Lettan, II, R. B., Scheidt, K. A., \& Van Duyne, R. P. A frequency domain existence proof of single-molecule surface-enhanced Raman spectroscopy. J. Am. Chem. Soc. 129, 16249-16256 (2007).

[109] Palmer, J. M. \& Grant, B. G. The Art of Radiometry. SPIE Press Bellingham, (2010).

[110] Karrer, E. The use of the Ulbricht sphere in measuring reflection and transmission factors. JOSA 5(1), 96-119 (1921).

[111] Porres, L., Holland, A., Pålsson, L.-O., Monkman, A. P., Kemp, C., \& Beeby, A. Absolute measurements of photoluminescence quantum yields of solutions using an integrating sphere. Journal of Fluorescence 16(2), 267-273 (2006). 
[112] Suzuki, K., Kobayashi, A., Kaneko, S., Takehira, K., Yoshihara, T., Ishida, H., Shiina, Y., Oishi, S., \& Tobita, S. Reevaluation of absolute luminescence quantum yields of standard solutions using a spectrometer with an integrating sphere and a back-thinned ccd detector. Physical Chemistry Chemical Physics 11(42), 9850-9860 (2009).

[113] Born, M. \& Wolf, E. Principles of Optics: Electromagnetic Theory of Propagation, Interference and Diffraction of Light. CUP Archive, (2000).

[114] Carr, K. F. Integrating sphere flux calculations. 1990 Catalog, (Labsphere, Inc., North Sutton N.H) , 84-86 (1990).

[115] Kirk, J. T. O. Modeling the performance of an integrating-cavity absorption meter: theory and calculations for a spherical cavity. Appl. Opt. 34, 4397-4408 (1995).

[116] Goebel, D. G. Generalized integrating-sphere theory. Applied Optics 6(1), 125-128 (1967).

[117] Fry, E. S., Kattawar, G. W., \& Pope, R. M. Integrating cavity absorption meter. Appl. Opt. 31, 2055-2065 (1992).

[118] Gaigalas, A. K., He, H.-J., \& Wang, L. Measurement of absorption and scattering with an integrating sphere detector: application to microalgae. J. Res. Natl. Inst. Stand. Technol. 114, 69-81 (2009).

[119] Babin, M. \& Stramski, D. Light absorption by aquatic particles in the near-IR spectral region. Limnol. Oceanogr. 47, 911-915 (2002).

[120] Tassan, S. \& Ferrari, G. M. Variability of light absorption by aquatic particles in the near-IR spectral region. Appl. Opt. 42, 4802-4810 (2003).

[121] Röttgers, R., Häse, C., \& Doerffer, R. Determination of the particulate absorption of microalgae using a point-source integrating-cavity absorption meter: verification with a photometric technique, improvements for pigment bleaching, and correction for chlorophyll fluorescence. Limnol. Oceanogr.: Methods 5, 1-12 (2007).

[122] Nelson, N. B. \& Prézelin, B. B. Calibration of an integrating sphere for determining the absorption coefficient of scattering suspensions. Appl. Opt. 32, 6710-6717 (1993). 
[123] Jávorfi, T., Erostyák, J., Gál, J., Buzády, A., Menczel, L., Garab, G., \& Naqvi, K. R. Quantitative spectrophotometry using integrating cavities. J. Photochem. Photobiol. B 82, 127 - 131 (2006).

[124] Hodgkinson, J., Masiyano, D., \& Tatam, R. P. Using integrating spheres as absorption cells: path-length distribution and application of beer's law. Applied optics 48(30), 5748-5758 (2009).

[125] Kochuveedu, S. T., Son, T., Lee, Y., Lee, M., Kim, D., \& Kim, D. H. Revolutionizing the fret-based light emission in core-shell nanostructures via comprehensive activity of surface plasmons. Scientific Reports $\mathbf{4}$ (2014).

[126] Zhao, L., Ming, T., Shao, L., Chen, H., \& Wang, J. Plasmon-controlled forster resonance energy transfer. The Journal of Physical Chemistry C 116(14), 8287-8296 (2012).

[127] Valeur, B. \& Berberan-Santos, M. N. Molecular Fluorescence: Principles and Applications. John Wiley \& Sons, (2012).

[128] Darby, B. L., Auguié, B., Meyer, M., Pantoja, A. E., \& Le Ru, E. C. Modified optical absorption of molecules on metallic nanoparticles at sub-monolayer coverage. Nature Photonics 10(1), 40-45 (2016).

[129] Geddes, C. D. \& Lakowicz, J. R. Editorial: Metal-enhanced fluorescence. Journal of Fluorescence 12(2), 121-129 (2002).

[130] Lakowicz, J. R., Geddes, C. D., Gryczynski, I., Malicka, J. B., Gryczynski, Z., Aslan, K., Lukomska, J., \& Huang, J. Advances in surfaceenhanced fluorescence. In Biomedical Optics 2004, 10-28. International Society for Optics and Photonics, (2004).

[131] Blackie, E. J., Ru, E. C. L., \& Etchegoin, P. G. Single-molecule surfaceenhanced Raman spectroscopy of nonresonant molecules. Journal of the American Chemical Society 131(40), 14466-14472 (2009).

[132] Goulet, P. J. \& Aroca, R. F. Non-resonant SERS from single molecules in langmuir-blodgett monolayers on nanostructured metallic films. Canadian Journal of Analytical Sciences and Spectroscopy 52(3), 172-177 (2007). 
[133] Hardin, B. E., Snaith, H. J., \& McGehee, M. D. The renaissance of dye-sensitized solar cells. Nature Photonics 6(3), 162-169 (2012).

[134] Qi, J., Dang, X., Hammond, P. T., \& Belcher, A. M. Highly efficient plasmon-enhanced dye-sensitized solar cells through metal@ oxide coreshell nanostructure. ACS NANO 5(9), 7108-7116 (2011).

[135] Vargas-Baca, I., Brown, A. P., Andrews, M. P., Galstian, T., Li, Y., Vali, H., \& Kuzyk, M. G. Linear and nonlinear optical responses of a dye anchored to gold nanoparticles dispersed in liquid and polymeric matrixes. Canadian Journal of Chemistry 80(11), 1625-1633 (2002).

[136] Ihara, M., Tanaka, K., Sakaki, K., Honma, I., \& Yamada, K. Enhancement of the absorption coefficient of cis-(ncs) 2 bis (2, 2'-bipyridyl-4, 4'-dicarboxylate) ruthenium (ii) dye in dye-sensitized solar cells by a silver island film. The Journal of Physical Chemistry B 101(26), 5153-5157 (1997).

[137] Xu, Q., Liu, F., Liu, Y., Cui, K., Feng, X., Zhang, W., \& Huang, Y. Broadband light absorption enhancement in dye-sensitized solar cells with Au-Ag alloy popcorn nanoparticles. Scientific Reports 3 (2013).

[138] Xu, Q., Liu, F., Meng, W., \& Huang, Y. Plasmonic core-shell metalorganic nanoparticles enhanced dye-sensitized solar cells. Optics Express 20(106), A898-A907 (2012).

[139] Londero, P. S., Leona, M., \& Lombardi, J. R. Definitive evidence for linked resonances in surface-enhanced Raman scattering: Excitation profile of $\mathrm{Cu}$ phthalocyanine. Applied Physics Letters 102(11), 111101 (2013).

[140] Huang, Y.-F., Zhu, H.-P., Liu, G.-K., Wu, D.-Y., Ren, B., \& Tian, Z.-Q. When the signal is not from the original molecule to be detected: chemical transformation of para-aminothiophenol on Ag during the SERS measurement. Journal of the American Chemical Society 132(27), 9244-9246 (2010).

[141] Saikin, S. K., Chu, Y., Rappoport, D., Crozier, K. B., \& Aspuru-Guzik, A. Separation of electromagnetic and chemical contributions to surface- 
enhanced Raman spectra on nanoengineered plasmonic substrates. The Journal of Physical Chemistry Letters 1(18), 2740-2746 (2010).

[142] Zayak, A., Hu, Y., Choo, H., Bokor, J., Cabrini, S., Schuck, P., \& Neaton, J. Chemical Raman enhancement of organic adsorbates on metal surfaces. Physical Review Letters 106(8), 083003 (2011).

[143] Otto, A., Lust, M., Pucci, A., \& Meyer, G. sers active sites, facts, and open questions. Canadian Journal of Analytical Sciences and Spectroscopy 52(3), 150-171 (2007).

[144] Lombardi, J. R. \& Birke, R. L. A unified approach to surface-enhanced Raman spectroscopy. The Journal of Physical Chemistry C 112(14), 5605-5617 (2008).

[145] Myers Kelley, A. Resonance Raman and resonance hyper-Raman intensities: Structure and dynamics of molecular excited states in solution. The Journal of Physical Chemistry A 112(47), 11975-11991 (2008).

[146] Tonks, D. \& Page, J. First-order resonance Raman profile lineshapes from optical absorption lineshapesa consistency test of standard theoretical assumptions. Chemical Physics Letters 66(3), 449-453 (1979).

[147] Page, J. B. \& Tonks, D. On the separation of resonance Raman scattering into orders in the time correlator theory. The Journal of Chemical Physics 75(12), 5694-5708 (1981).

[148] Brafman, O., Chan, C., Khodadoost, B., Page, J., \& Walker, C. Resonance Raman scattering study of azulene. i. experiment and theoretical analysis via transform techniques. The Journal of Chemical Physics 80(11), 5406-5417 (1984).

[149] Stallard, B., Callis, P., Champion, P., \& Albrecht, A. Application of the transform theory to resonance Raman excitation profiles in the soret region of cytochrome-c. The Journal of Chemical Physics 80(1), 70-82 (1984).

[150] Schlather, A. E., Large, N., Urban, A. S., Nordlander, P., \& Halas, N. J. Near-field mediated plexcitonic coupling and giant Rabi splitting in individual metallic dimers. Nano Lett. 13, 3281-3286 (2013). 
[151] Zengin, G., Johansson, G., Johansson, P., Antosiewicz, T. J., Käll, M., \& Shegai, T. Approaching the strong coupling limit in single plasmonic nanorods interacting with J-aggregates. Sci. Rep. 3, 3074 (2013).

[152] Törmä, P. \& Barnes, W. Strong coupling between surface plasmon polaritons and emitters: a review. Reports on Progress in Physics 78(1), 013901 (2015).

[153] Yoshie, T., Scherer, A., Hendrickson, J., Khitrova, G., Gibbs, H., Rupper, G., Ell, C., Shchekin, O., \& Deppe, D. Vacuum rabi splitting with a single quantum dot in a photonic crystal nanocavity. Nature 432(7014), 200-203 (2004).

[154] Craighead, H. G. \& Glass, A. Optical absorption of small metal particles with adsorbed dye coats. Optics Lett. 6, 248-250 (1981).

[155] Haes, A. J., Zou, S., Zhao, J., Schatz, G. C., \& Van Duyne, R. P. Localized surface plasmon resonance spectroscopy near molecular resonances. J. Am. Chem. Soc. 128, 10905-10914 (2006).

[156] Zhao, J., Jensen, L., Sung, J., Zou, S., Schatz, G. C., \& Van Duyne, R. P. Interaction of plasmon and molecular resonances for rhodamine 6G adsorbed on silver nanoparticles. J. Am. Chem. Soc. 129, 7647-7656 (2007).

[157] Ni, W., Yang, Z., Chen, H., Li, L., \& Wang, J. Coupling between molecular and plasmonic resonances in freestanding dye-gold nanorod hybrid nanostructures. J. Am. Chem. Soc. 130, 6692-6693 (2008).

[158] Ni, W., Chen, H., Su, J., Sun, Z., Wang, J., \& Wu, H. Effects of dyes, gold nanocrystals, $\mathrm{pH}$, and metal ions on plasmonic and molecular resonance coupling. J. Am. Chem. Soc. 132, 4806-4814 (2010).

[159] Ni, W., Ambjörnsson, T., Apell, S. P., Chen, H., \& Wang, J. Observing plasmonic-molecular resonance coupling on single gold nanorods. Nano Lett. 10, 77-84 (2010).

[160] Ambjörnsson, T., Mukhopadhyay, G., Apell, S. P., \& Käll, M. Resonant coupling between localized plasmons and anisotropic molecular coatings in ellipsoidal metal nanoparticles. Physical Review B 73(8), 085412 (2006). 
[161] Antosiewicz, T. J., Apell, S. P., \& Shegai, T. Plasmon-exciton interactions in a core-shell geometry: From enhanced absorption to strong coupling. ACS Photonics 1, 454-463 (2014).

[162] Faucheaux, J. A., Fu, J., \& Jain, P. K. Unified theoretical framework for realizing diverse regimes of strong coupling between plasmons and electronic transitions. J. Phys. Chem. C 118, 2710-2717 (2014).

[163] Wiederrecht, G. P., Wurtz, G. A., \& Hranisavljevic, J. Coherent coupling of molecular excitons to electronic polarizations of noble metal nanoparticles. Nano Lett. 4, 2121-2125 (2004).

[164] Fofang, N. T., Park, T.-H., Neumann, O., Mirin, N. A., Nordlander, P., \& Halas, N. J. Plexcitonic nanoparticles: Plasmon-exciton coupling in nanoshell-J-aggregate complexes. Nano Lett. 8, 3481-3487 (2008).

[165] Davis, T. J., Gómez, D. E., \& Vernon, K. C. Interaction of molecules with localized surface plasmons in metallic nanoparticles. Phys. Rev. B 81, 045432 (2010).

[166] Valmorra, F., Bröll, M., Schwaiger, S., Welzel, N., Heitmann, D., \& Mendach, S. Strong coupling between surface plasmon polariton and laser dye Rhodamine 800. Appl. Phys. Lett. 99, 051110 (2011).

[167] Chen, H., Shao, L., Woo, K. C., Wang, J., \& Lin, H.-Q. Plasmonicmolecular resonance coupling: plasmonic splitting versus energy transfer. J. Phys. Chem. C 116, 14088-14095 (2012).

[168] Fang, Y., Blinn, K., Li, X., Weng, G., \& Liu, M. Strong coupling between rhodamine $6 \mathrm{G}$ and localized surface plasmon resonance of immobile Ag nanoclusters fabricated by direct current sputtering. Appl. Phys. Lett. 102, 143112 (2013).

[169] Cacciola, A., Di Stefano, O., Stassi, R., Saija, R., \& Savasta, S. Ultrastrong coupling of plasmons and excitons in a nanoshell. ACS Nano 8, 11483-11492 (2014).

[170] Chantharasupawong, P., Tetard, L., \& Thomas, J. Coupling enhancement and giant Rabi-splitting in large arrays of tunable plexcitonic substrates. J. Phys. Chem. C 118(41), 23954-23962 (2014). 
[171] Anker, J. N., Hall, W. P., Lyandres, O., Shah, N. C., Zhao, J., \& Van Duyne, R. P. Biosensing with plasmonic nanosensors. Nature materials 7(6), 442-453 (2008).

[172] Mayer, K. M. \& Hafner, J. H. Localized surface plasmon resonance sensors. Chemical Reviews 111(6), 3828-3857 (2011).

[173] Ruemmele, J. A., Hall, W. P., Ruvuna, L. K., \& Van Duyne, R. P. A localized surface plasmon resonance imaging instrument for multiplexed biosensing. Analytical Chemistry 85(9), 4560-4566 (2013).

[174] Chen, S., Svedendahl, M., Käll, M., Gunnarsson, L., \& Dmitriev, A. Ultrahigh sensitivity made simple: nanoplasmonic label-free biosensing with an extremely low limit-of-detection for bacterial and cancer diagnostics. Nanotechnology 20(43), 434015 (2009).

[175] Xu, H. \& Käll, M. Surface-plasmon-enhanced optical forces in silver nanoaggregates. Physical Review Letters 89(24), 246802 (2002).

[176] Kasha, M. Energy transfer mechanisms and the molecular exciton model for molecular aggregates. Radiation Research 20(1), 55-70 (1963).

[177] Kerker, M. The optics of colloidal silver: something old and something new. Journal of Colloid and Interface Science 105(2), 297-314 (1985).

[178] Matousek, P., Clark, I., Draper, E., Morris, M., Goodship, A., Everall, N., Towrie, M., Finney, W., \& Parker, A. Subsurface probing in diffusely scattering media using spatially offset Raman spectroscopy. Applied spectroscopy 59(4), 393-400 (2005).

[179] Derkacs, D., Chen, W., Matheu, P., Lim, S., Yu, P., \& Yu, E. Nanoparticle-induced light scattering for improved performance of quantum-well solar cells. Applied Physics Letters 93(9), 091107 (2008).

[180] Catchpole, K. \& Polman, A. Design principles for particle plasmon enhanced solar cells. Applied Physics Letters 93(19), 191113 (2008).

[181] Dignam, M. J. \& Moskovits, M. Optical properties of sub-monolayer molecular films. J. Chem. Soc., Faraday Trans. 2 69, 56-64 (1973). 
[182] Chandrasekharan, N., Kamat, P. V., Hu, J., \& Jones, G. Dyecapped gold nanoclusters: photoinduced morphological changes in gold/Rhodamine 6G nanoassemblies. The Journal of Physical Chemistry B 104(47), 11103-11109 (2000).

[183] Nasr, C., Liu, D., Hotchandani, S., \& Kamat, P. V. Dye-capped semiconductor nanoclusters. excited state and photosensitization aspects of Rhodamine 6G H-aggregates bound to $\mathrm{SiO} 2$ and $\mathrm{SnO} 2$ colloids. The Journal of Physical Chemistry 100(26), 11054-11061 (1996).

[184] Narband, N., Uppal, M., Dunnill, C. W., Hyett, G., Wilson, M., \& Parkin, I. P. The interaction between gold nanoparticles and cationic and anionic dyes: enhanced UV-visible absorption. Physical Chemistry Chemical Physics 11(44), 10513-10518 (2009).

[185] Shrivastava, R., Jain, B., \& Das, K. Spectroscopic investigations on the binding of Methylene Blue and Nile Blue to negatively charged gold nanorods. J. Mol. Struct. 1020, 56 - 62 (2012).

[186] Morton, S. M. \& Jensen, L. A discrete interaction model/quantum mechanical method for describing response properties of molecules adsorbed on metal nanoparticles. J. Chem. Phys. 133 (2010).

[187] Angeloni, L., Smulevich, G., \& Marzocchi, M. Resonance Raman spectrum of Crystal Violet. Journal of Raman Spectroscopy 8(6), 305-310 (1979).

[188] Lovell, S., Marquardt, B. J., \& Kahr, B. Crystal Violets shoulder. Journal of the Chemical Society, Perkin Transactions 2 11, 2241-2247 (1999).

[189] Campo, J., Painelli, A., Terenziani, F., Van Regemorter, T., Beljonne, D., Goovaerts, E., \& Wenseleers, W. First hyperpolarizability dispersion of the octupolar molecule Crystal Violet: Multiple resonances and vibrational and solvation effects. Journal of the American Chemical Society 132(46), 16467-16478 (2010).

[190] A lengthy discussion of the issue can be found in the blog article: $\quad$ http://condensedconcepts.blogspot.co.nz/2012/02/who-caresabout-crystal-violet.html. 
[191] Dietze, D. R. \& Mathies, R. A. Molecular orientation and optical properties of 3,3-diethylthiatricarbocyanine iodide adsorbed to gold surfaces: Consequences for surface-enhanced resonance Raman spectroscopy. J. Phys. Chem. C 119, 9980-9987 (2015).

[192] Forker, R., Gruenewald, M., \& Fritz, T. Optical differential reflectance spectroscopy on thin molecular films. Annu. Rep. Prog. Chem., Sect. C: Phys. Chem. 108, 34-68 (2012).

[193] Sun, G. \& Khurgin, J. Origin of giant difference between fluorescence, resonance, and nonresonance Raman scattering enhancement by surface plasmons. Phys. Rev. A 85, 063410 (2012).

[194] Leng, W. \& Vikesland, P. J. MGITC facilitated formation of AuNP multimers. Langmuir 30(28), 8342-8349 (2014).

[195] M. J. Frisch et al. Gaussian 09 Revision D.01. Gaussian Inc. Wallingford CT 2009.

[196] Perdew, J. P., Burke, K., \& Ernzerhof, M. Generalized gradient approximation made simple. Phys. Rev. Lett. 77, 3865-3868 (1996).

[197] Adamo, C. \& Barone, V. Toward reliable density functional methods without adjustable parameters: The PBE0 model. J. Chem. Phys. 110, 6158-6170 (1999).

[198] Weigend, F. \& Ahlrichs, R. Balanced basis sets of split valence, triple zeta valence and quadruple zeta valence quality for $\mathrm{H}$ to Rn: Design and assessment of accuracy. Phys. Chem. Chem. Phys. 7, 3297-3305 (2005).

[199] Weigend, F. Accurate coulomb-fitting basis sets for $\mathrm{H}$ to Rn. Phys. Chem. Chem. Phys. 8, 1057-1065 (2006).

[200] Kittel, C. Introduction to Solid State Physics. Wiley, (2005).

[201] Johnson, P. B. \& Christy, R.-W. Optical constants of the noble metals. Physical Review B 6(12), 4370 (1972). 